\title{
Esquemas numéricos para equações hiperbólicas e aplicações
}

\author{
Marcio Demetrius Martinez
}

Orientador:

\section{Prof. Dr. José Alberto Cuminato}

Dissertação apresentada ao Instituto de Ciências Matemáticas e de Computação ICMC-USP, como parte dos requisitos para obtenção do titulo de Mestre em Ciências de Computação e Matemática Computacional.

\section{USP - São Carlos \\ Fevereiro/2002}

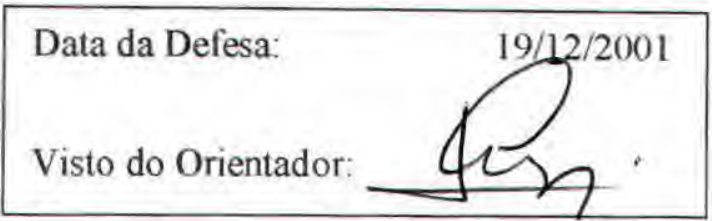


Ninguém seduza a si mesmo: Se alguém entre vós pensa que é sábio neste sistema de coisas, torne-se ele tolo, para que se torne sabio. Pois a sabedoria deste mundo é tolice perante Deus; porque está escrito:

"Ele apanha os sábios na sua própria astúcia”. E novamente: "Jeová sabe que os raciocínios dos sábios são füteis".

1 Coríntios 3: 18-20

Dedico essa dissertação de mestrado aos meus familiares: meus

pais, Waldomiro e Silvia, aos meus irmãos, Marcelo e

Cristiane, e ao meu cunhado Anderson, que ao

longo desses dois anos contribuiram com

amor, paciência e encorajamento

para o término desse

trabalho. 


\section{Agradecimentos}

Sou grato a todos que de maneira direta ou indireta contribuiram para o meu sucesso e colaboraram nessa minha jornada. Desses, gostaria de agradecer às seguintes pessoas:

À Jeová Deus por ter-me dado vida e saúde.

Ao professor José Alberto Cuminato pela orientação, confiança e disposição em ajudar durante todo o curso.

Aos professores Marcelo José Saia e Vanderlei M. do Nascimento pelas recomendações.

Aos meus amigos Denise, Edivaldo e Helton, que me acompanham desde a graduação e que sempre estiveram presentes, nestes dois anos de estudos, dando apoio e amizade.

Aos colegas da Pós, especialmente à turma de Matemática Computacional de 1999, Fernando, Helton, Juliana. Luciana, Magda, Marcelo, Rúbia e Wellington, que compartilharam comigo os momentos difíceis e também as alegrias e vitórias.

Também quero agradecer a CNPq, pelo apoio financeiro.

Muito obrigado a todos! 


\section{Resumo}

Neste trabalho estudamos esquemas numéricos para resolver as formulações de valor de fronteira e de valor inicial para uma frente em movimento. Nosso objetivo é motivar e apresentar esquemas baseados nas relaçōes existentes entre frentes em propagação, equações de Hamilton-Jacobi e leis de conservação hiperbólicas.

Quando uma frente inicial evolui no tempo através de uma das formulações hiperbólicas, podem surgir singularidades, cúspides e mudanças em sua topologia e assim faz-se necessário a compreensão das técnicas de discretização de leis de conservação hiperbólicas para a obtenção de esquemas numéricos capazes de tratar e descrever corretamente esses problemas na geometria da frente. A solução numérica das leis de conservação inclui o desenvolvimento de esquemas numéricos capazes de resolver choques, descontinuidades e escolher a solução entrópica entre as muitas soluções fracas existentes. Para isso, analisamos esquemas na forma conservativa com propriedades especiais, tais como, esquemas Upwind, Monótonos, TVD, Entropia, Limitante de fluxo e Limitante de inclinação. Esses esquemas são acompanhados com uma coleção de implementações. Essa teoria pode ser empregada para o rastreamento da interface de escoamentos multifásicos, e uma aplicação futura que estamos interessados é a determinação da fronteira de um domínio a partir de seus pontos interiores para aplicação no simulador de escoamentos multifásicos na área de mecânica de fluidos. 


\section{Abstract}

In this work we study schemes for solving the boundary and initial value formulations of front motion. Our objective is to motivate and present schemes based on the link between evolving fronts, Hamilton-Jacobi equations and hyperbolic conservation laws.

When a initial front evolves in time through the hyperbolic formulation, singularities and changes in its topology can arise, thus making it necessary the comprehension of discretization technics of hyperbolic conservation laws for obtaining numerical schemes capable of resolving shocks, descontinuities and selecting the entropy solution from all the existent weak solutions. With this purpose in mind, we analised conservative schemes with special properties, such as, Upwind, Monotone, TVD, Entropy, Flux-Limiter and Slope-Limiter are analysed. These schemes are followed by a collection of implemented examples.

This theory can be used for tracking the interface of multifase flows. Future applications that we are interested in include the determination of the boundary of a domain from its interior points for application in a simulator of multifase flows in the area of fluid mechanics. 


\section{Sumário}

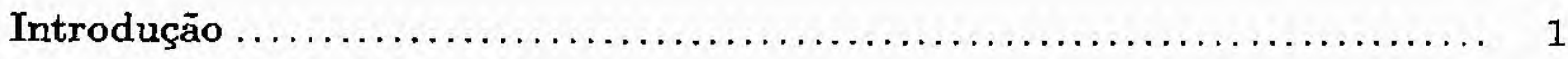

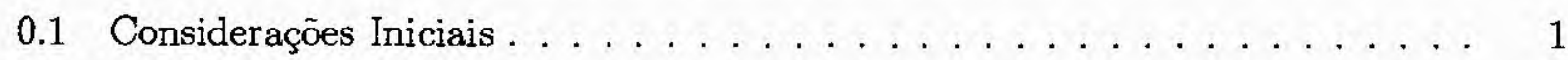

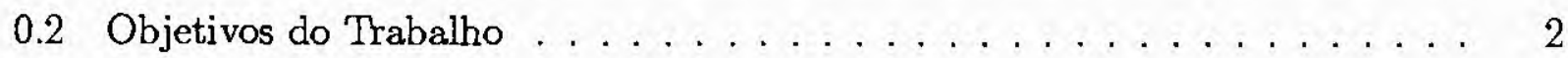

0.3 Conteúdo do Trabalho $\ldots \ldots \ldots \ldots \ldots \ldots \ldots \ldots$

1 Formulação de Propagação de Interfaces 5

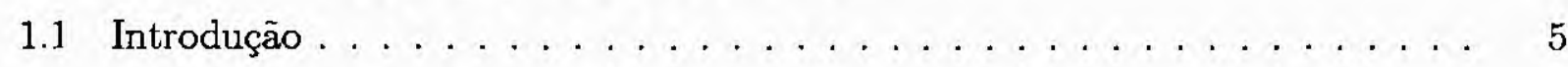

1.2 Formulação de Valor de Fronteira . . . . . . . . . . . . . 6

1.3 Formulação de Valor Inicial $\ldots \ldots \ldots \ldots \ldots \ldots$

2 Análise da Evolução de Frentes $\quad 13$

2.1 Introdução . . . . . . . . . . . . . . . . 13

2.2 Equações Paramétricas . . . . . . . . . . . . . . . . . . . 13

2.3 Efeitos da Curvatura: O Limite Viscoso e a Relação com Leis de Conservação

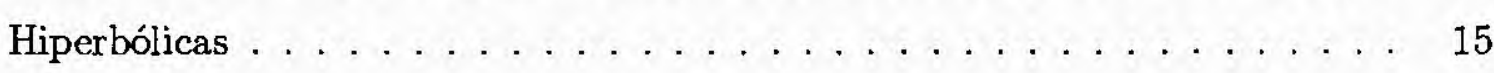

2.4 Discretização Direta das Equações Paramétricas . . . . . . . . . . . 21

3 Teoria e Esquemas Numéricos para Equações Hiperbólicas $\quad 27$

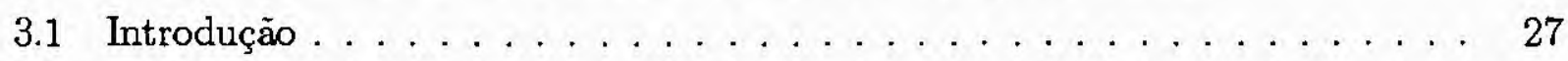

3.2 Breve Introdução à Teoria de Equações Hiperbólicas . . . . . . . . . . . 28

3.2.1 Propagação de Singularidades e Choques . . . . . . . . . . . . 29

3.2.2 Função de Fluxo, Condições de Entropia e Soluções Fracas . . . . . 31

3.3 Esquemas Numéricos para Leis de Conservação . . . . . . . . . . . . 43

3.3 .1 Esquemas Upwind . . . . . . . . . . . . . . 48

3.3.2 Esquemas de Godunov e de Engquist-Osher . . . . . . . . . 50

3.3.3 Esquemas de Segunda Ordem de Lax-Wendroff e de Beam-Warming . 60 
3.3 .4 Definições e Resultados . . . . . . . . . . . . . . . . 63

3.3.5 Forma Incremental e Viscosidade Numérica . . . . . . . . . . . . 67

3.3 .6 Principais Teoremas . . . . . . . . . . . . . . . . . 69

3.3 .7 Esquemas de Alta Resolução . . . . . . . . . . . . . . . . . 83

3.3 .8 Métodos Limitantes de Fluxo . . . . . . . . . . . . . . . . 84

3.3 .9 Métodos Limitante de Inclinação . . . . . . . . . . . . . . 95

4 Esquemas Hiperbólicos e Equaçōes de Hamilton-Jacobi 105

4.1 Introdução . . . . . . . . . . . . . . . . . . . 105

4.2 Esquemas Unidimensionais . . . . . . . . . . . . . . . 106

4.3 Esquemas Numéricos em Dimensões Maiores . . . . . . . . . . . . . . . 109

4.4 O Problema de Valor Inicial: Método dos Conjuntos de Níveis . . . . . . 109

4.5 Esquemas para Funçōes Velocidades Não-Convexas . . . . . . . . . . . 117

4.6 O Problema de Valor de Fronteira: Método Estacionário . . . . . . . . . . . 119

4.7 Aproximaçōes para as Variáveis Geométricas . . . . . . . . . . . . . . 119

5 Resultados e Consideraçōes Finais $\quad 123$

5.1 Transporte de uma Frente Através de um Campo Parabólico . . . . . . . . 123

5.2 Transporte da Curva Cosseno . . . . . . . . . . . . . . . 126

5.3 Rotação de um Círculo Utilizando Esquemas de Primeira e Segunda Ordem . 128

5.4 Considerações Finais . . . . . . . . . . . . . . . . . . . . . . . . . . . . . 129

5.4 .1 Conclusões . . . . . . . . . . . . . . . . 129

5.4 .2 Perspectivas Futuras . . . . . . . . . . . . . . . . 131

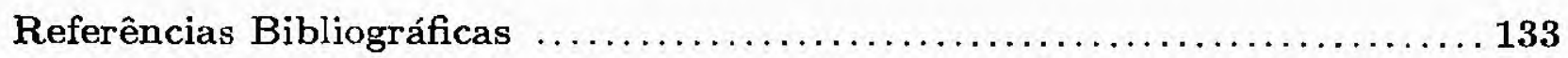




\section{Introdução}

\subsection{Considerações Iniciais}

O problema da propagação de interfaces ocorre em uma ampla variedade de áreas do conhecimento e possui inúmeras aplicações importantes. Dentre algumas áreas que podemos citar estão: Física, Química, Mecânica de fluidos, Combustão, Processamento de imagens, Ciências dos materiais, Fabricação de componentes microeletrônicos, Visão computacional, Teoria do controle, Sismologia (Geologia), e uma coleção de outras áreas. As aplicações mais importantes que podemos mencionar estão na área de modelagem geométrica, que inclui a determinação da fronteira de um domínio a partir do conhecimento de pontos interiores, cálculo da distância entre curvas e superfícies, determinação da forma a partir dả sombra e o problema do caminho mínimo, entre outras.

Um dos temas importantes em ciência aplicada é o desenvolvimento de métodos para aproximar grandezas que variam e cujo cálculo exato é difícil de se obter. No mundo físico que vivemos podemos distinguir fenômenos que evoluem no tempo (transientes) e aqueles que estâo em um estado de equilíbrio (estacionários). Neste trabalho, nos concentraremos em problemas transientes ou de propagação que envolvem a variação temporal das grandezas físicas que estão sendo consideradas. A partir de um tempo inicial t to avançamos até um tempo final $t_{f}$ em sucessivos intervalos de tempo $\Delta t$. Nota-se que estamos caminhando no tempo desde $t_{0}$ a $t_{f}$, e por isso, problemas transientes são também denominados "problemas de marcha" (em inglês, marching problems). As técnicas nas quais nos basearemos neste trabalho para o estudo de interfaces em movimento envolvem os Métodos de Marcha Rápida e Conjuntos de Níveis, idealizados por James A. Sethian e Stanley Osher. Estas, sāo técnicas fundalmentalmente diferentes que servem para rastrear, analizar e calcular o movimento de interfaces. Essas frentes são movimentadas em suas direçōes normais como resultado de uma função velocidade conhecida $F$, que frequentemente depende da curvatura 
da frente. À medida que essas frentes evoluem no tempo, podem surgir singularidades, cúspides e mudanças na topologia. As equações diferenciais parciais que governam essa evolução são hiperbólicas c portanto, singularidades na curvatura comportam-se como os choques na teoria das leis de conservação. Consequentemente, a teoria usada para estudar os choques nas leis de conservação será importante no tratamento de singularidades em frentes. Os métodos de marcha rápida resultam da modelagem do movimento como um problema de valor de fronteira e têm como equação modeladora a equação Eikonal, enquanto que, os métodos de conjuntos de níveis resultam dessa modelagem como um problema de valor inicial e seu estudo envolve a análise das equaçōes de Hamilton-Jacobi. Será estudado também a relação existente entre as equações de Hamilton-Jacobi e leis de conservação hiperbólicas. Essas duas perspectivas são equivalentes somente para equações unidimensionais, mas apesar disso, a metodologia numérica pode ser usada para esquemas multidimensionais para ambos pontos de vista da propagação de uma interface.

A teoria das equações hiperbólicas oferece dificuldades as mais diversas. No sentido de obter resultados práticos, a pesquisa nessa área evoluiu na direção das equações na forma de lei de conservação, onde consegue-se estabelecer resultados importantes principalmente no que concerne à compreensão de choques. Os estudos da teoria das leis de conservação data de 1950, conforme o trabalho de E. Hopf [22], seguido por trabalhos de P. D. Lax [24] e O. Oleinik [27]. O estudo de soluções descontínuas avançou com o surgimento da noção de soluçōes fracas e com o progresso na teoria matemática de distribuições.

\subsection{Objetivos do Trabalho}

Como já mencionamos, uma das principais dificuldades que surge ao resolver uma equação diferencial parcial na forma conservativa é que sua solução não necessariamente é diferenciável, mesmo quando os dados iniciais o são. O fato da solução apresentar singularidades, leva à idéia de relaxarem-se as exigências para que uma dada função seja solução, permitindo-se soluções não diferenciáveis, ou fracas.

Ao fazermos essa concessão, introduz-se o fenômeno de múltiplas soluções, sendo que obviamente, apenas uma deve representar o problema físico. Dessa forma, faz-se necessário a introdução da noção de soluçōes fracas apropriadas que representem corretamente o problema físico considerado. Na teoria das equaçōes diferenciais, a solução apropriada é a chamada 
solução entrópica. Resta agora, o problema de como projetar métodos numéricos que sejam capazes de escolher a solução fraca entrópica dentre as demais.

Este trabalho de mestrado tcm como objetivo principal o estudo de técnicas numéricas que, naturalmente, sejam capazes de tratar a não diferenciabilidade e também escolham a solução entrópica. Essas técnicas serão utilizadas na construção de esquemas numéricos

eficientes e precisos para ambas as formulações da evolução de uma interface a fịm de que estas admitam soluções fisicamente corretas.

Os métodos para equações hiperbólicas serão utilizados para aproximar as equações para o rastreamento de uma frente em propagação. Concentrar-nos-emos, neste trabalho, na formulação do problema de valor inicial.

\subsection{Conteúdo do Trabalho}

Gostaríamos de ser bastante claros quanto aos objetivos deste trabalho. Nossa ênfase será o estudo de métodos numéricos para equações hiperbólicas, que sejam capazes de escolher a solução entrópica. Entretanto, para motivar e possivelmente sugerir novas áreas de aplicação, escolhemos estudar essas técnicas numéricas em paralelo com uma dessas possíveis aplicações que é o da evolução de uma interface. Com esse objetivo, apresentamos no capítulo 1 uma breve introdução à formulação do movimento de uma interface como problema de valor de fronteira e inicial para uma equação hiperbólica. No capítulo 2, através das equações paramétricas, mostramos como a evolução de uma curva pode desenvolver singularidades.

O capítulo 3 é a parte principal deste trabalho, onde estudamos vários aspectos dos métodos numéricos para leis de conservação. No capítulo 4, os esquemas estudados no capítulo 3 são adaptados para ambas formulações, as quais são equações hiperbólicas de Hamilton-Jacobi; e finalmente, no capítulo 5, alguns exemplos simples de movimentos de curvas e superfícies são apresentados através de esquemas derivados da formulação de valor inicial. 


\section{Capítulo 1}

\section{Formulação de Propagação de}

\section{Interfaces}

\subsection{Introdução}

Neste capítulo descrevemos a modelagem da propagação de uma curva inicial como um problema de valor de fronteira e também como um problema de valor inicial. Além disso, mostramos as técnicas que usaremos para o rastreamento de interfacrs ou frentes quando elas se movimentam. O leitor interessado poderá obter informaçōes complementares nas seguintes referências: [29], [35, 37, 38], utilizadas na confecção desse capítulo.

Considere uma fronteira, uma curva em duas dimensões ou uma superfície em três, delimitando uma região, com uma parte externa e outra interna, conforme mostrado na Figura 1.1.1. Imagine que esta curva/superfície move-se na direção normal a si mesma com função velocidade $F$ conhecida.

De maneira geral, a função $F$, que pode depender de vários parâmetros, pode ser escrita como

$$
F=F(L, G, I)
$$

em que

- $L=$ Propriedades Locais, determinadas pelas informaçōes geométricas locais, tais como curvatura e direção normal.

- $G=$ Propriedades Globais da frente, dependem da forma e posição da frente. 
- I = Propriedades Independentes, independem da forma da frente, tais como a velocidade do fluido que transporta a frente.

Um dos principais desafios na modelagem de problemas de interfaces em movimento está em produzir um modelo adequado para a função $F$. Neste capítulo, assumimos que a função velocidade $F$ é conhecida. Dada $F$ e a posição inicial de uma interface, o objetivo é rastrear o movimento dessa interface ao longo do tempo. Nossa primeira tarefa é formular um modelo desse movimento do ponto de vista Euleriano, isto é, aquele em que o sistema de coordenadas permanece fixo.

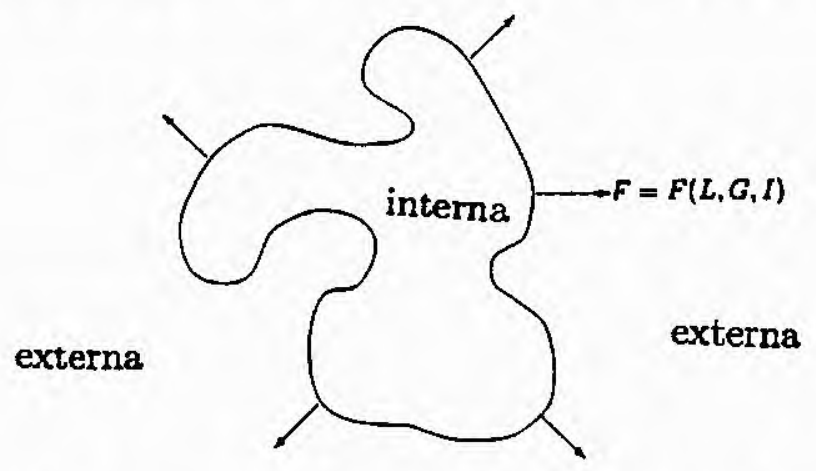

Figura 1.1.1. Curva propagando com velocidade $F$ na direção normal.

\subsection{Formulação de Valor de Fronteira}

Assumindo que $F>0$, temos que a frente se move para "fora". Uma maneira de caracterizar a posição desta frente é calcular o tempo de chegada $T(x, y)$, tomado a partir da posiçāo inicial quando esta cruza cada ponto $(x, y)$ da malha, como mostrado na Figura 1.2.1.

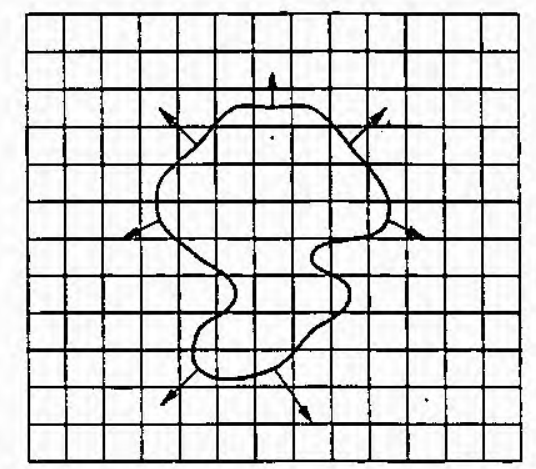

Figura 1.2.1. Cálculo do tempo de chegada em $(x, y)$ para uma frente se expandindo com velocidade $F>0$.

A equação para $T(x, y)$ é derivada da seguinte maneira: Em uma dimensão, usando o fato de que a velocidade é a taxa instantânea da variação do espaço $x$ em relação a qualquer instante $T$, temos 


$$
\frac{d x}{d T}=F \rightarrow F \frac{d T}{d x}=1 .
$$

Em duas dimensões, seja $T(x, y)$ uma função de classe $C^{2}$ no aberto $A \subset \mathbb{R}^{2}$ e sejam $(x, y) \in A$ e $(h, k) \neq(0,0)$ tais que o segmento de extremidades $(x, y)$ e $(x+h, y+k)$ esteja contido em $A$. Nestas condições

$$
T(x+h, y+k)=T(x, y)+\frac{\partial T(x, y)}{\partial x} h+\frac{\partial T(x, y)}{\partial y} k+E(h, k),
$$

em que $E(h, k) \equiv o\left(h^{2}, k^{2}\right)$. Se desprezarmos o erro $E$ então a aproximação

$$
|T(x+h, y+k)-T(x, y)| \approx\left|\frac{\partial T(x, y)}{\partial x} h+\frac{\partial T(x, y)}{\partial y} k\right|=|<\nabla T(x, y),(h, k)>|,
$$

será melhor quanto menor for $|(h, k)|$.

Se o vetor incremento $(h, k)$ for tomado na mesma direção de $\nabla T$, que é ortogonal ao conjunto de nível de $T$ no ponto $(x, y)$, pela desigualdade de Cauchy-Schwartz, temos:

$$
|<\nabla T(x, y),(h, k)>|=\|\nabla T(x, y)\|\|(h, k)\| .
$$

(Aqui || || é a norma Euclideana). Assim

$$
|T(x+h, y+k)-T(x, y)| \approx\|\nabla T(x, y)\|\|(h, k)\|
$$

e desta maneira,

$$
1 \approx \frac{\|\nabla T(x, y)\|\|(h, k)\|}{|T(x+h, y+k)-T(x, y)|}
$$

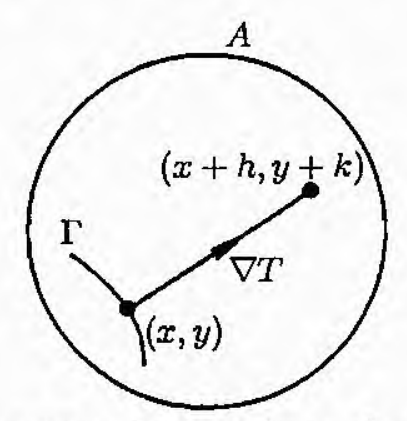

Sabendo que a velocidade $F$ é a taxa instantânea da variaçào da distância pela variação do tempo, temos que

$$
1=\lim _{(h, k) \rightarrow(0,0)}\left\{\|\nabla T(x, y)\| \frac{\|(h, k)\|}{|T(x+h, y+k)-T(x, y)|}\right\}=\|\nabla T(x, y)\| F,
$$

e portanto,

$$
\|\nabla T\| F=1, \quad T=0 \text { sobre } \Gamma,
$$


em que $\Gamma$ é a localização inicial da interface.

Dessa maneira, o movimento é caracterizado pela solução de um problema de valor de fronteira. Se a velocidade $F$ depende somente da posição, então essa equação reduz-se ao que é conhecido como equação Eikonal. Como exemplo, a Figura 1.3 .1 mostra a superfície tempo de chegada $T(x, y)$ para uma frente circular expandindo com velocidade unitária. Se a função velocidade é positiva (negativa), então o tempo de chegada da frente $T(x, y)$ é único.

Se $F$ varia de sinal, a frente pode mover-se para frente e depois para trás. passando duas vezes por um mesmo ponto, nesse caso $T(x, y)$ não é unicamente definida. Dessa forma, a formulação de fronteira está totalmente justificada quando o movimento da frente dá-se em apenas uma direção, não permitindo sua reversão. Essa é uma hipótese bastante restritiva no caso do movimento da interface entre dois fluidos em escoamentos bifásicos, pois seu movimento pode ser geral. Daí a necessidade de uma formulação mais robusta, como veremos a seguir.

\subsection{Formulação de Valor Inicial}

Nessa seção, suporemos que a frente move-se com uma velocidade genérica $F$, que pode assumir valores positivos e/ou negativos. Devemos então considerar o fato de que a frente pode mover-se para frente ou para trás, e portanto, pode passar por um ponto $(x, y)$ várias vezes, permitindo à função tempo de chegada assumir vários valores. A maneira de resolver esse problema é associar a posição inicial da frente ao conjunto de nível zero de uma função de dimensão maior $\phi$ conforme mostrado na Figura 1.3.2.

A principal diferença entre as Figuras 1.3 .1 e 1.3 .2 reside no fato de que em 1.3.1 o eixo vertical representa a função $T(x, y)$ enquanto em 1.3 .2 o eixo vertical representa a variável independente $t$, e portanto o gráfico de $\phi(x, y, t)$ está num espaço de dimensão maior em relação ao gráfico de $T(x, y)$, sendo este último uma superfície de nível de $\phi$.

Para derivar uma equação do movimento para a funçāo $\phi$, supomos que em cada instante $t$ cada ponto $(x, y)$ da curva inicial siga uma trajetória, esteja localizado em $X(t)=(x(t), y(t))$, e pertença ao conjunto de nível zero da função $\phi$, isto é:

$$
\phi(X(t), t)=0
$$

Note que o parâmetro $t$, não parametriza a curva no plano- $(x, y)$ ele apenas determina a 
nova posição desse ponto, conforme ilustrado no capítulo seguinte pela Figura 2.2.1.

Diferenciando a equação (1.2) temos, $\phi_{t}+\nabla \phi(X(t), t) . X^{\prime}(t)=0$. Se $F$ é a velocidade na direção normal, então $X^{\prime}(t) \cdot \vec{n}=F$, em que $\vec{n}=\nabla \phi /|\nabla \phi|$, e o gradiente dc $\phi$ ć calculado nas variáveis $x$ e $y$ considerando a variável $t$ constante. Obtemos assim uma equação de evolução para $\phi$. a saber

$$
\begin{gathered}
\phi_{t}+F|\nabla \phi|=0 \\
\phi(X, t=0) \text { dado. }
\end{gathered}
$$

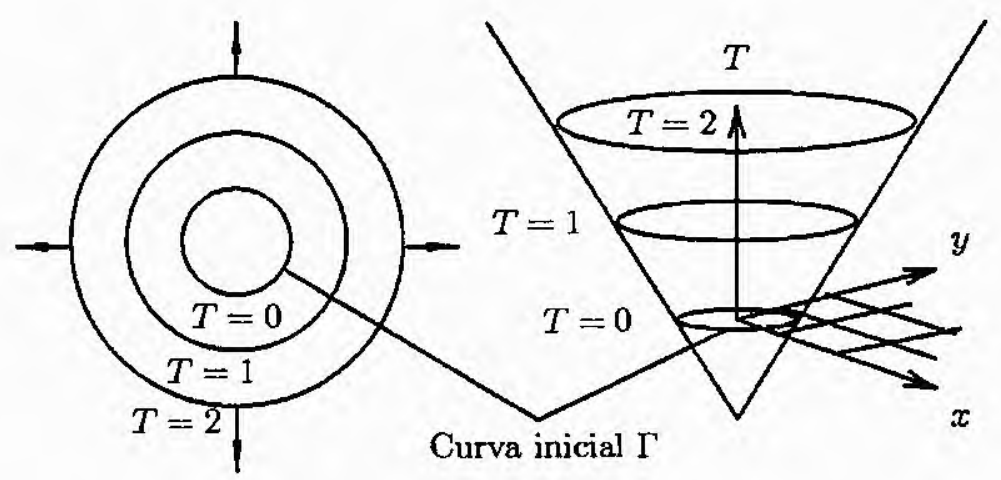

Figura 1.3.1. Transformação do movimento da frente num problema de valor de fronteira.
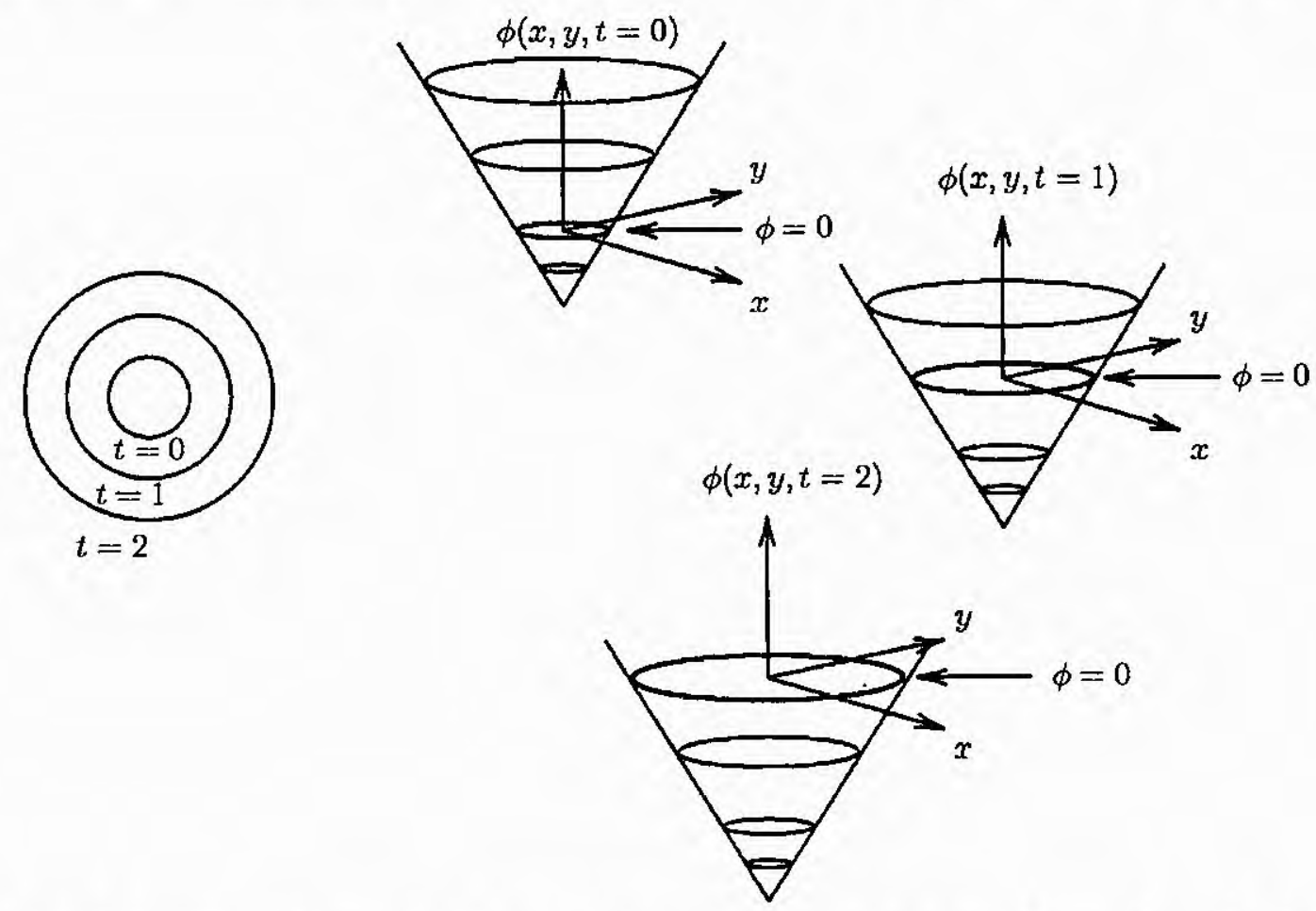

Figura 1.3.2. Transformação do movimento da frente num problema de valor inicial.

Por exemplo, no caso ilustrado pela Figura 1.3.2, a função $\phi$ pode ser expressa por $\phi(x, y, t)=\left(x^{2}+y^{2}\right)^{1 / 2}-r-t$. Quando $t=0$, a curva inicial (círculo de raio $r$ ) coincide 
com o conjunto de nível zero de $\phi$. Similarmente mostramos para dois tempos posteriores as respectivas posições da função de nível $\phi$.

Resumindo, seja $\Gamma$ uma curva no plano propagando em uma direção normal a si mesma com velocidade $F$ tal que $\Gamma(t)$ dá a posição da frente no tempo $t$. Desta forma, podemos formular essa evolução de duas maneiras distintas à saber:

problema de valor de fronteira

$$
|\nabla T| F=1
$$

$$
\begin{aligned}
& \text { Frente }= \Gamma(t)=\{(x, y): T(x, y)=t\} \\
& \text { restrição } F>0
\end{aligned}
$$

\section{problema de valor inicial}

$$
\begin{gathered}
\phi_{t}+F|\nabla \phi|=0 \\
\text { Frente }=\Gamma(t)=\{(x, y): \phi(x, y, t)=0\} \\
F \text { geral }
\end{gathered}
$$

Observação 1.3.1. Em capítulos posteriores, estaremos concentrados em produzir esquemas numéricos que aproximem a solução de ambas as formulações hiperbólicas. Em geral, a solução de equações hiperbólicas pode não ser diferenciável e assim é preciso entender a solução em um sentido local e mais fraco. Além disso, essa solução fraca pode não ser única e um critério para escolher uma dessas soluções fracas faz-se necessário. Um possível critério é transformar a equação hiperbólica em uma equação diferencial parabólica, pela adição de um termo de viscosidade, por exemplo, para o problema de valor inicial em 1 dimensão temos

$$
\phi_{t}+F|\nabla \phi|=\epsilon u_{x x}
$$

em que $\epsilon>0$ é um parâmetro.

A equação parabólica possui uma única solução $\phi_{\epsilon}(x, t)$ regular. A solução $\phi(x, t)$ do problema hiperbólico seria então o limite de $\phi_{\epsilon}(x, t)$ quando $\epsilon \rightarrow 0$ se este existir. Essa solução é denominada solução entrópica para o problema hiperbólico. Essa teminologia vem do fato de que a entropia pode somente aumentar ao cruzar um choque na dinâmica dos gases e que (1.4) é um mecanismo que pode ser usado para identificar a solução que possui esta propriedade. No que se segue, estaremos sempre mencionando a palavra entropia tanto no contexto de propagação de frentes como no de equações hiperbólicas e o leitor deve associá-la com esse processo de limite, que fornecerá a solução do problema físico em questão.

Existem certas vantagens associadas com essas duas perspectivas.

- Ambas são facilmente generalizadas para dimensões maiores, isto é, para superfícies propagando em très dimensôes ou mais.

- Mudanças topológicas na frente $\Gamma$ são tratadas naturalmente. A posição da frente no tempo $t$ é dada ou pelo nível zero $\phi(x, y, t)=0$ da função de nível $\phi$ ou pelo nível $T(x, y)=t$ 
da soluçâo do problema de valor de fronteira. Quando uma frente evolui no tempo ela pode romper-se e/ou unir-se, mas, em ambos os casos, o fato importante é que a solução de valor de fronteira $T(x, y)$ e a função de nível $\phi$ cuicarão de ŕcpresentar esses fenòmenos sem a necessidade de tratamento específico. Esse fato apenas ilustra a versatilidade c robustez dos métodos de conjunto ċe nívcl e de marcha rápida.

- Propriedades geométricas intrínsicas da frente são facilmente determinadas em ambas as formulaçōes. Por exemplo, em qualquer ponto da frente, seu vetor normal é dado por $\vec{n}=\nabla \phi /|\nabla \phi|$ ou $\vec{n}=\nabla T /|\nabla T|$, e sua curvatura é dada por:

$$
\kappa=\left\{\begin{array}{l}
\nabla \frac{\nabla \phi}{\nabla \nabla \phi}=\frac{\phi_{x x x} \phi_{y}^{2}-2 \phi_{x} \phi_{y} \phi_{x y}+\phi_{y y} \phi_{x}^{2}}{\left(\phi_{x}^{2}+\phi_{y}^{2}\right)^{3 / 2}} \\
\nabla \frac{\nabla T}{\mid \nabla T_{\mid}}=\frac{T_{x x} T_{y}^{2}-2 T_{x} T_{y} T_{x y}+T_{y y} T_{x}^{2}}{\left(T_{x}^{2}+T_{y}^{2}\right)^{3 / 2}} .
\end{array}\right.
$$

Apresentamos assim, as duas equaçòes principais de movimento. No Capítulo 2 estudaremos os problemas da propagação de frentes ilustrando as dificuldades com exemplos. 


\section{Capítulo 2}

\section{Análise da Evolução de Frentes}

\subsection{Introdução}

Neste capítulo formulamos as equaçōes paramétricas de movimento de uma curva propagando-se ao longo de seu campo vetorial normal com velocidade $F$ dependendo de sua curvatura. Estudamos sua estabilidade, e mostramos que cantos (singularidades na curvatura) desenvolvem quando a frente evolui. Mostramos que esses cantos são análogos a choques na solução de leis de conservação hiperbólicas e que através da noção de uma solução fraca que satisfaz entropia (ver Observação 1.3.1) pode-se obter uma solução que melhor representa a física do problema. Assim sendo, o objetivo deste capítulo é mostrar as dificuldades que podem surgir na propagação, como o aparecimento de singularidades. Essas dificuldades são mais facilmente entendidas utilizando-se a formulação paramétrica, que é uma formulação mais simples e direta.

As referências principais utilizadas neste capítulo foram as seguintes: [29], [36, 37, 38, 39], citadas na bibliografia.

\subsection{Equações Paramétricas}

Seja $\gamma$ uma curva simples, suave e fechada em $\mathbb{R}^{2}$, e seja $\gamma(t)$ uma familia de 1-parâmetro gerada movendo-se $\gamma$ ao longo de seu campo vetorial normal com velocidade $F$, em que $F$ é uma função escalar da curvatura $\kappa$ de $\gamma$. Seja $X(s, t)=(x(s, t), y(s, t))$ o vetor posição que parametriza $\gamma(t)$ em $s, \quad 0 \leq s \leq S, \quad X(0, t)=X(S, t)$. Assumiremos que a curva está parametrizada no sentido anti-horário. Sejam $\vec{n}(s, t)$ e $\kappa(s, t)$ as parametrizações do vetor 
normal e da curvatura em $X(s, t)$, respectivamente. A primeira equação de movimento é:

$$
\begin{aligned}
X_{t}(s, t) \cdot \vec{n}(s, t) & =F(\kappa(s, t)), \\
X(s, 0) & =\gamma(0) ; \quad s \in[0, S], \quad t \in[0, \infty) .
\end{aligned}
$$

Como o vetor $\vec{n}$ tem $\|\vec{n}\|=1$ e os vetores $X_{t}(s, t)$ e $\vec{n}(s, t)$ possuem a mesma direção (são linearmente dependentes), então da equação (2.1) temos que $F \vec{n}=\left(X_{t} \cdot \vec{n}\right) \vec{n}=X_{t}(\vec{n} . \vec{n})$, e portanto $X_{t}=F \vec{n}$. Assim sendo, temos uma outra equação paramétrica de movimento que, escrita em termos de suas componentes, é dada por

$$
\begin{aligned}
& x_{t}=F\left(\frac{y_{s s} x_{s}-x_{s s} y_{s}}{\left(x_{s}^{2}+y_{s}^{2}\right)^{\frac{3}{2}}}\right) \frac{y_{s}}{\left(x_{s}^{2}+y_{s}^{2}\right)^{\frac{1}{2}}}, \\
& y_{t}=-F\left(\frac{y_{s s} x_{s}-x_{s s} y_{s}}{\left(x_{s}^{2}+y_{s}^{2}\right)^{\frac{3}{2}}}\right) \frac{x_{s}}{\left(x_{s}^{2}+y_{s}^{2}\right)^{\frac{1}{2}}} \\
& (x(s, 0), y(s, 0))=\gamma(0) \quad 0 \leq s \leq S,
\end{aligned}
$$

em que as expressōes da curvatura e do vetor normal são: $\kappa=\frac{y_{s a} x_{s}-x_{s a} y_{A}}{\left(x_{s}^{2}+y_{s}^{2}\right)^{\frac{3}{2}}}$ e $\vec{n}=\frac{\left(y_{a},-x_{s}\right)}{\left(x_{s}^{2}+y_{s}^{2}\right)^{\frac{1}{2}}}$, respectivamente. Esta é uma representação Lagrangeana devido a imagem de $(x(s, t), y(s, t))$ descrever o movimento da frente.

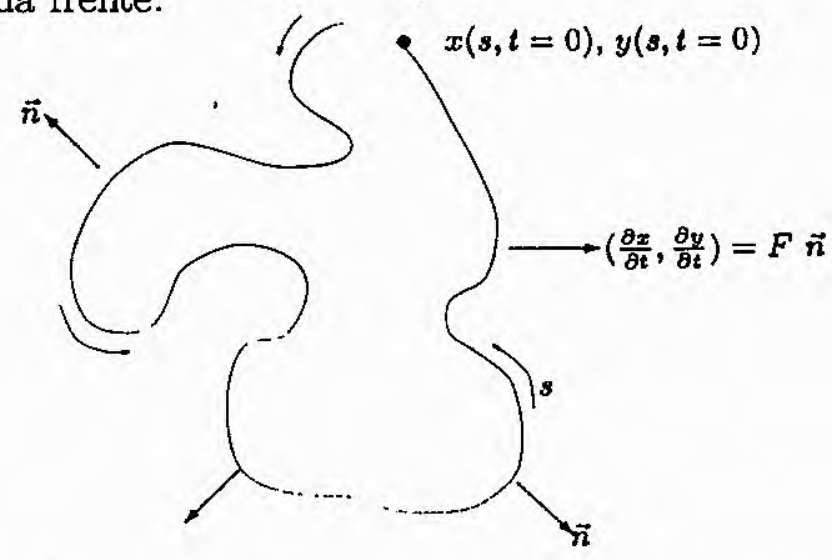

Figura 2.2.1. Parametrização de uma curva se propagando.

A equaçāo (2.2) representa a formulação paramétrica do movimento de uma curva. Através dessa formulação estudaremos a seguir várias características da propagação e suas relaçōes com propriedades das equações hiperbólicas. Mostraremos por exemplo que cantos são análogos aos choques nas leis de conservação hiperbólicas, e que as técnicas usadas nas lei de conservação para o estudo de choques podem também ser empregadas para frentes em movimento. 
Para melhor entender os mecanismos envolvidos na evolução de uma frente, precisamos de alguns resultados, que passamos a apresentar.

\subsection{Efeitos da Curvatura: O Limite Viscoso e a Relação com Leis de Conservação Hiperbólicas}

Oscilaçōes em uma curva fechada podem ser caracterizadas por mudanças de sinal da curvatura. Tais oscilaçōes contribuem para formar cantos na curva quando ela evolui. A evolução, como já dissemos, dar-se-á ao longo de seu campo normal com velocidade $F$ que dependerá da curvatura da frente.

Se $\alpha(s)$ corresponde à função comprimento de arco, então $d \alpha=g(s, t) d s$, em que $g(s, t)=$ $\left(x_{s}^{2}+y_{s}^{2}\right)^{1 / 2}$ é a métrica que mede a deformação da parametrização. Definimos a oscilação total (também conhecida como variação total) da frente por:

$$
\operatorname{Var}(t)=\int_{0}^{s}|\kappa(s, t)| g(s, t) d s
$$

(Sem o módulo na curvatura, $\operatorname{Var}(t)=2 \pi$ sempre). O valor absoluto faz com que essa função meça a quantidade de enrugamento da curva. O objetivo é ver se este enrugamento cresce ou decresce quando a frente se movimenta; ver Figura 2.3.1.

Diferenciando a curvatura e a métrica em relação a $t$, junto com a substituição de (2.2), produz as equaçōes de evoluçào paramétricas correspondentes para a curvatura e métrica , a saber,

$$
\begin{aligned}
\kappa_{t} & =-g^{-1}\left(F_{s} g^{-1}\right)_{s}-\kappa^{2} F, \quad g^{-1}=\frac{1}{g} \\
g_{t} & =g \kappa F .
\end{aligned}
$$

Suponha que temos uma curva inicial propagando com velocidade $F(\kappa)$ a qual permanece suave em seu movimento. O resultado abaixo relaciona o aumento/decrescimento da variação total com a função velocidade de propagação.

Proposição 2.3.1. Considere uma frente movendo-se ao longo do seu campo vetorial normal com velocidade $F(\kappa)$, como na Equação (2.2). Assuma que a curva inicial $\gamma(t=0)$ é simples, suave e nâo convexa, tal que $\kappa(s, 0)$ muda de sinal. Assuma também que $F$ é duas vezes diferenciável e que $\kappa(s, t)$ é duas vezes diferenciável para $0 \leq s \leq S$ e $0 \leq t \leq T$. Então, para $0 \leq t \leq T$, 
- se $F_{\kappa}(0) \leq 0\left(F_{\kappa}(0) \geq 0\right)$, então

$$
\frac{d \operatorname{Var} \overline{(t)}}{d t} \leq 0 \quad\left(\frac{d \operatorname{Var}(t)}{d t} \geq 0\right)
$$

- se $F_{\kappa}(0)<0\left(F_{\kappa}(0)>0\right)$ e $\kappa_{s}(0) \neq 0$ então

$$
\frac{d \operatorname{Var}(t)}{d t}<0 \quad\left(\frac{d \operatorname{Var}(t)}{d t}>0\right) .
$$

Demonstração: Suponha que em $s_{1}(t), \ldots, s_{i}(t), \ldots, s_{n}(t), \kappa\left(s_{i}(t), t\right)=0$ e troca de sinal de $s<s_{i}(t)$ a $s>s_{i}(t)$. Assuma que $\kappa>0$ em $\left(s_{1}(t), s_{2}(t)\right),\left(s_{3}(t), s_{4}(t)\right),\left(s_{n-1}, s_{n}(t)\right)$ e $\kappa<0$ em $\left(s_{2}(t), s_{3}(t)\right),\left(s_{4}(t), s_{5}(t)\right),\left(s_{n}(t), s_{1}(t)+S=s_{1}(t)\right)$. Então

$\operatorname{Var}(t)=\int_{0}^{S}|\kappa| g d s=\int_{s_{1}(t)}^{s_{2}(t)} \kappa g d s+\int_{s_{3}(t)}^{s_{4}(t)} \kappa g d s+\ldots+\int_{s_{n-1}(t)}^{s_{n}(t)} \kappa g d s-\int_{s_{2}(t)}^{s_{3}(t)} \kappa g d s-\int_{s_{4}(t)}^{s_{5}(t)} \kappa g d s-$ $\ldots-\int_{s_{n}(t)}^{s_{1}(t)+S} \kappa g d s$.

Por simplicidade assuma que $\kappa$ muda de sinal somente em dois pontos, $s_{1}(t)$ e $s_{2}(t)$, então

$$
\begin{aligned}
\frac{d \operatorname{Var}(t)}{d t .}= & \frac{d}{d t}\left(\int_{s_{1}(t)}^{s_{2}(t)} \kappa g d s-\int_{s_{2}(t)}^{s_{1}(t)+S} \kappa g d s\right)=\int_{s_{1}(t)}^{s_{2}(t)}(\kappa g)_{t} d s-\int_{s_{2}(t)}^{s_{1}(t)+S}(\kappa g)_{t} d s \\
& +\kappa\left(s_{2}(t), t\right) g\left(s_{2}(t), t\right) s_{2}^{\prime}(t)-\kappa\left(s_{1}(t), t\right) g\left(s_{1}(t), t\right) s_{1}^{\prime}(t) \\
& -\kappa\left(s_{1}(t)+S, t\right) g\left(s_{1}(t)+S, t\right)\left(s_{1}(t)+S\right)^{\prime}+\kappa\left(s_{2}(t), t\right) g\left(s_{2}(t), t\right) s_{2}^{\prime}(t)
\end{aligned}
$$

em que ambas as notações usadas, $\frac{d}{d t}$ e $/$, referem-se à diferenciação em $t$. Por hipótese, $\kappa\left(s_{1}(t), t\right)=\kappa\left(s_{2}(t), t\right)=\kappa\left(s_{1}(t)+S, t\right)=0, \operatorname{assim} \frac{d V a r(t)}{d t}=\int_{s_{1}(t)}^{s_{2}(t)}\left(\kappa_{t} g+\kappa g_{t}\right) d s-\int_{s_{2}(t)}^{s_{1}(t)+S}\left(\kappa_{t} g+\right.$ $\left.\kappa g_{t}\right) d s$. Usando as equações (2.4) e (2.5), temos

$$
\begin{aligned}
\frac{d V a r(t)}{d t} & =\int_{s_{1}(t)}^{s_{2}(t)}\left(-\left(g^{-1} F_{s}\right)_{s}-g \kappa^{2} F+g \kappa^{2} F\right) d s-\int_{s_{2}(t)}^{s_{1}(t)+S}\left(-\left(g^{-1} F_{s}\right)_{s}-g \kappa^{2} F+g \kappa^{2} F\right) d s \\
& =-\int_{s_{1}(t)}^{s_{2}(t)}\left(g^{-1} F_{s}\right)_{s} d s+\int_{s_{2}(t)}^{s_{1}(t)+S}\left(g^{-1} F_{s}\right)_{s} d s \\
& =-\left.2\left(g^{-1} F_{\kappa} \kappa_{s}\right)\right|_{s_{2}(t)}+\left.2\left(g^{-1} F_{\kappa} \kappa_{s}\right)\right|_{s_{1}(t)} .
\end{aligned}
$$

Por hipótese, $\kappa>0$ para $s_{1}(t)<s<s_{2}(t)$, portanto $\left.\kappa_{s}\right|_{s_{1}(t)} \geq 0$ e $\left.\kappa_{s}\right|_{s_{2}(t)} \leq 0$. Suponha $F_{\kappa}(0) \geq 0$. Então, como $g^{-1}>0$, ambos os termos à direita da equação (2.8) são não negativos e $\frac{d \operatorname{Var}(t)}{d t} \geq 0$. Inversamente, se $F_{\kappa}(0) \leq 0$, então ambos os termos são não positivos e $\frac{d V a r(t)}{d t} \leq 0$. Se $F_{\kappa}$ é estritamente menor ou maior que zero e $\kappa_{s} \neq 0$ em $s_{1}(t)$ e $s_{2}(t)$, então as desigualdades são também estritas.

A proposição garante que se $F_{\kappa}<0$ em $\kappa=0$ então a variação total diminui quando a frente move-se. tornando-a mais suave. No caso especial em que $\gamma(t)$ é convexa para todo $t$, a proposição é trivial, visto que $\operatorname{Var}(t)=\int_{0}^{S} \kappa(s, t) g(s, t) d s=2 \pi$. O corolário abaixo aplica-se a uma frente movendo-se com veiocidade constante. 
Corolário 2.3.2. Se $F_{\kappa}=0$, então a variação total é constante.

Como exemplo, considere o círculo de raio $r$ parametrizado por $\alpha(s)=(r \cos (s), r \operatorname{sen}(s))$ $s \in[0,2 \pi]$. Sua curvatura é dada por $\kappa(s)=1 / r$ e $g(s)=r$, e portanto, $\operatorname{Var}(t)=2 \pi$. Também para a elipse de equação $\frac{x^{2}}{a^{2}}+\frac{y^{2}}{b^{2}}=1$, parametrizada por $\beta(s)=(a \cos (s), b \operatorname{sen}(s))$, $s \in[0,2 \pi]$ sua curvatura é dada por $\kappa(s)=\frac{a b}{\left(a^{2} \operatorname{sen}^{2}(s)+b^{2} \cos ^{2}(s)\right)^{3 / 2}}$ e $g(s)=\left(a^{2} \operatorname{sen}^{2}(s)+\right.$ $\left.b^{2} \cos ^{2}(s)\right)^{1 / 2}$. Com ajuda de algum software especializado para o cálculo de integrais, pois a integral a ser resolvida não é trivial, pode ser constatado que $\operatorname{Var}(t)=2 \pi$.

A mudança na variação total da frente é mostrado depender de $F_{\kappa}$ somente onde $\kappa$ muda de sinal. Dois casos frequentemente utilizados para a função $F$ podem ser utilizados como ilustração. Considere a função velocidade $F(\kappa)=1-\epsilon \kappa$ para $\epsilon>0$. Sua derivada é $F_{\kappa}=-\epsilon$, e portanto, a variação total diminui. Inversamente, para $F(\kappa)=1+\epsilon \kappa$, temos derivada positiva, e consequentemente essas oscilações aumentam contribuindo para o aparecimento de cantos na frente.

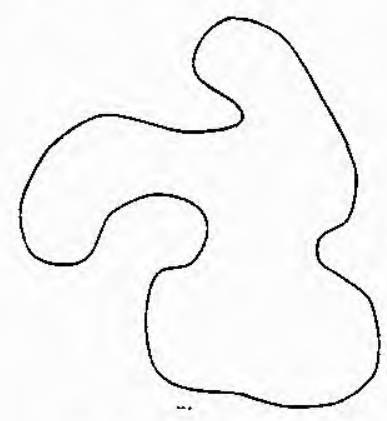

Curva original

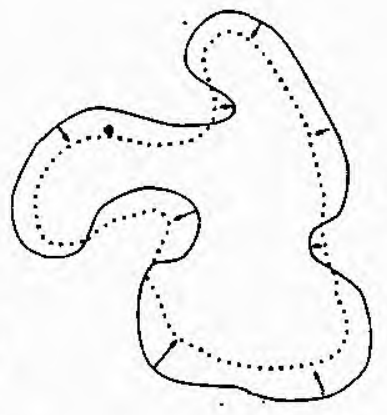

Diminuição na variação

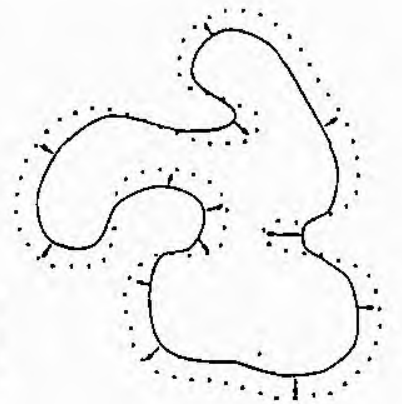

Aumento na variação

Figura 2.3.1. Mudança na variação $\operatorname{Var}(t)$.

Considere uma função velocidade da forma $F=1-\epsilon \kappa$, onde $\epsilon$ é uma constante. Os efeitos da variação do termo $\epsilon \kappa$ são significativos, e preparam o terreno para a construção de esquemas numéricos exatos que aderem à condição de entropia. Considerando a equação de evolução dada por (2.4), ela pode ser reescrita como

$$
\kappa_{t}=\epsilon \kappa_{\alpha \alpha}+\epsilon \kappa^{3}-\kappa^{2}
$$

em que a segunda derivada da curvatura é tomada com respeito ao comprimento de arco $\alpha$ para eliminar a métrica $g$. Esta é uma equação parabólica de reação-difusão; o termo de reação $\left(\epsilon \kappa^{3}-\kappa^{2}\right)$ tende a produzir singularidades que são balanceadas pelo efeito de suavização do termo de difusão $\left(\epsilon \kappa_{\alpha \alpha}\right)$. Entretanto, $\operatorname{com} \epsilon=0$, temos uma equação 
unicamente de reação $\kappa_{t}=-\kappa^{2}$ e o desenvolvimento de singularidades na solução exata $\kappa(s, t)=\kappa(s, 0) /(1+t \kappa(s, 0))$, pode surgir em algum tempo finito $t$ se a curvatura da curva inicial for sempre negativa. Vemos assim que, a propagação de uma curva com $F=1$, pode desenvolver cantos.

Como exemplo, considere a curva inicial cosseno dada por

$$
\gamma(0)=(1-s,[1+\cos (2 \pi s)] / 2)
$$

propagando com velocidade $F(\kappa)=1-\epsilon \kappa, \epsilon>0$. Quando a frente evolui, a depressão em $s=n+1 / 2$ é realçada pelo termo de reação (pois $\kappa<0$ em tais pontos) e suavizada pelo termo de difusão positivo. Para $\epsilon>0$, foi mostrado, $[36,29]$, que a frente permanece $C^{\infty}$ e para $\epsilon=0$ a depressão é realçada com o passar do tempo. Esta depressão corresponde ao desenvolvimento de um canto na frente.

Verifiquemos agora o caso $\bar{\epsilon}=0$ para o exemplo (2.10), e portanto, a frente estará propagando-se com velocidade $F(\kappa)=1$. A parametrização é escolhida de tal maneira que o interior está à esquerda quando movemos na direção de crescimento de $s$. Utilizando as equações de movimento, (2.2), obtemos a solução:

$$
\begin{aligned}
& x(s, t)=\frac{y_{s}(s, t=0)}{\left(x_{s}^{2}(s, t=0)+y_{s}^{2}(s, t=0)\right)^{1 / 2}} t+1-s, \\
& y(s, t)=\frac{-x_{s}(s, t=0)}{\left(x_{s}^{2}(s, t=0)+y_{s}^{2}(s, t=0)\right)^{1 / 2}} t+\frac{1+\cos (2 \pi s)}{2} .
\end{aligned}
$$

Como mencionamos acima, enquanto a frente se movimenta surgem singularidades em tempo finito. Quando isto acontece, a normal é ambiguamente definida e não fica claro como continuar a evolução. Essas ambiguidades estão relacionadas com descontinuidades na solução. Podemos no entanto, levar o processo adiante definindo uma estratégia de escolha da normal, eliminando as ambiguidades. Resulta desse processo o que conhecemos como a obtenção da solução fraca, uma vez que, por não ser diferenciável, esta não pode satisfazer a equação diferencial.

Uma possível solução fraca é a solução rabo de andorinha (swallowtail) que permite à frente produzir auto interseção. Esta solução é obtida das Equações (2.11) e (2.12) e ilustrada na Figura 2.3.3. Entretanto, essa solução fraca ainda apresenta ambiguidade, e só a eliminaremos com a remoção do "rabo"do "rabo de andorinha". Isto é possível pois a solução para este problema no tempo $t$ pode ser construída avançando cada ponto da frente em sua direção normal uma distância $t$, a qual corresponde a distância mais curta 
ou "primeira chegada" (este procedimento é mencionado na literatura como o princípio de construção de Huygens, para mais detalhes veja [36]).

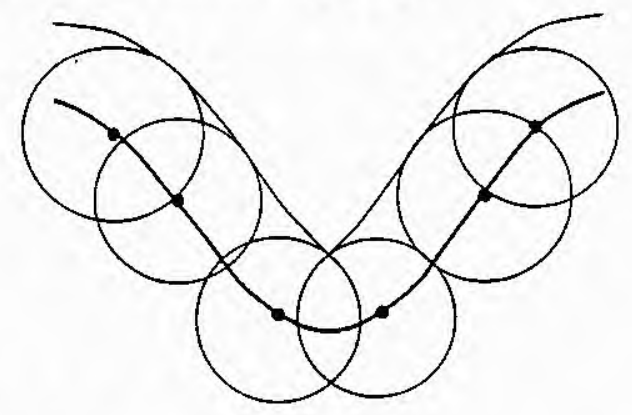

Figura 2.3.2. Solução de Huygens para $F=1$.

Com o princípio de Huygens ou, equivalentemente, através da seguinte condição de entropia proposta por Sethian [36], se imaginamos uma curva inicial como uma fonte para uma chama propagar, então a chama propagando satisfaz a exigência de que uma vez que um ponto no domínio é incendiado pela frente expandindo, ele permanece incendiado, as ambiguidades são removidas. Esta construção fornece o princípio de Huygens que satisfaz entropia dada na Figura 2.3.2.

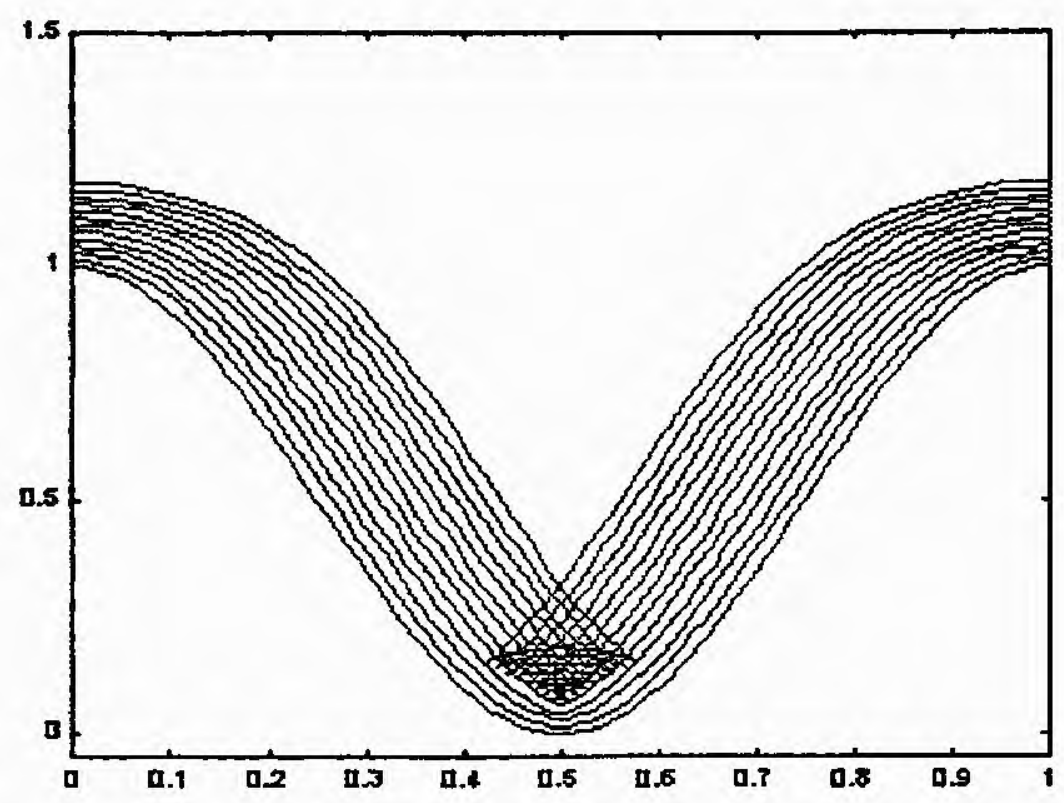

Figura 2.3.3. Curva cosseno propagando com velocidade unitária. Rabo de andorinha.

Que semelhança existe entre esta "condiçāo de entropia" com a noção de "entropia"? Enquanto uma resposta mais precisa será dada no Capítulo 3 , intuitivamente "entropia" refere-se a organização de informação. Em termos gerais, uma condição de entropia é algo que diz que nenhuma informação nova pode ser criada durante a evolução do problema. Além do 
mais, o exemplo mostra que uma vez que a condição de entropia é exigida, alguma informação contida nos dados iniciais é perdida. De fato, a condição de entropia, "uma vez que uma partícula é incendiada, ela permanece incendiada", significa que uma vez que um canto desenvolve-se, a solução não é mais reversível para a eliminação desse canto. O problema não pode voltar no tempo; se tentarmos fazê-lo, o dado inicial não será recuperado. Assim, alguma informação sobre a solução será definitivamente perdida.

A solução fraca satisfazendo entropia é o limite das soluções suaves $(\epsilon>0)$ quando o termo da curvatura se anula. Essa afirmação é a observação central, chave para a noção de soluçōes de entropia na propagação de frentes.

A seguir, verificaremos que os problemas apresentados acima na propagação de frentes são análogos ao desenvolvimento de choques em leis de conservação hiperbólicas. Considere uma equação para $v(x, t)$ da forma

$$
v_{t}+[G(v)]_{x}=0
$$

conhecida como uma "lei de conservação hiperbólica". Um exemplo simples é a equação de Burgers

$$
v_{t}+v v_{x}=0
$$

que descreve o movimento de um fluido compressível em 1-dimensão. A soluçāo dessa equaçāo pode desenvolver singularidades, conhecidas como "choques". Esses choques podem aparecer mesmo se os dados iniciais forem suaves. Para encontrarmos as soluções de (2.14) podemos recorrer ao conhecido método das características ou à parabolização da equação, que consiste em adicionar um termo conhecido como viscosidade artificial $\left(\epsilon v_{x x}\right)$ em (2.14), a saber,

$$
v_{t}+v v_{x}=\epsilon v_{x x}
$$

Para qualquer $\epsilon>0$, pode ser mostrado que a equação (2.15) é parabólica e que esta segunda derivada atua como um termo de suavização nas soluções e cessa o aparecimento de tais choques. Desta maneira, a solução $v^{\epsilon}(x, t)$ de $(2.15)$ é sempre suave $\lim _{\epsilon \rightarrow 0} v^{\epsilon}(x, t)=$ $v(x, t)$. As soluçōes fracas nãọ são únicas em geral e o processo de passagem ao limite fornece uma lei para a escolha daquela que melhor representa a física do problema. Dizemos que neste caso a solução fraca satisfaz a condição de entropia. 
Uma pergunta natural é a seguinte: Qual é a relação desses fatos com a propagação de frentes?

Considere uma frente inicial dada pelo gráfico de uma função $f(x)$, com $f$ e $f^{\prime}$ periódicas em $[0,1]$, e suponha que a frente enquanto se propaga permaneça o gráfico de uma função para todo tempo. Seja $\psi(x, t) \circ$ deslocamento da função em propagação, e portanto, $\psi(x, 0)=$ $f(x)$. De (1.3) temos,

$$
\psi_{t}=F\left(1+\psi_{x}^{2}\right)^{\frac{1}{2}}
$$

Usando $F=1-\epsilon \kappa$ e a fórmula $\kappa=-\psi_{x x} /\left(1+\psi_{x}^{2}\right)^{3 / 2}$, temos

$$
\psi_{t}-\left(1+\psi_{x}^{2}\right)^{\frac{1}{2}}=\epsilon \frac{\psi_{x x}}{1+\psi_{x}^{2}}
$$

A equação (2.17) é uma equação diferencial parcial de primeira ordem no espaço e no tempo, e com um termo de segunda ordem no lado direito. Diferenciando-a na variável $x$ em ambos os lados temos uma equação de evolução para a inclinação $u=d \psi / d x$ da frente, a saber,

$$
u_{t}+\left[-\left(1+u^{2}\right)^{\frac{1}{2}}\right]_{x}=\epsilon\left[\frac{u_{x}}{1+u^{2}}\right]_{x}
$$

Como observamos, a equação (2.18) é uma lei de conservação hiperbólica viscosa, com $G(u)=-\left(1+u^{2}\right)^{1 / 2}$, para a inclinação $u$. Assim, podemos fazer a analogia da condição de entropia para frentes em movimento com a da propagação de choques na teoria de leis de conservação hiperbólicas.

Nossa meta é então desenvolver esquemas de propagaçăo de frentes baseados nessas idéias de curvatura/viscosidade. (veja Capítulo 4)

\subsection{Discretização Direta das Equações Paramétricas}

Esta seção tem por objetivo mostrar que a discretização simplista das equações paramétricas nāo produz resultados satisfatórios, tanto do ponto de vista de confiabilidade, como do ponto de vista do tempo de processamento. De fato, técnicas das partículas marcadoras, que é uma outra técnica numérica para rastrear interfaces, mostraram ser instáveis em regiões onde a curvatura aumenta, a não ser que passos muito pequenos no tempo forem utilizados para assegurar estabilidade. 


\section{Métodos Marcadores}

Uma abordagem simplista para modelar frentes vem da discretização da forma Lagrangeana das equações de movimento dada por (2.2). Nesta abordagem, a parametrização é discretizada em um conjunto de partículas marcadoras cujas posições em qualquer tempo são usadas para reconstruir a frente. Esta é conhecida por uma variedade de nomes, tais como, técnicas de partículas marcadoras, métodos string, e métodos nodais. Em duas dimensões, a frente pode ser reconstruída por segmentos de retas, já em três dimensões, triângulos são frequentemente usados.

Podemos ilustrar essa técnica através de um esquema simples que constrói uma aproxịmação de diferenças finitas para as equações de movimento. Divida o intervalo de parametrização $[0, S]$ em $M$ intervalos iguais de tamanho $\Delta s$, fornecendo $M+1$ pontos da malha $s_{i}=i \Delta s, i=0, \ldots, M$, como mostrado na Figura 2.4.1. Da mesma forma, divida o tempo em intervalos iguais de comprimento $\Delta t$. A imagem de cada ponto da malha $i \Delta S$ em cada passo no tempo $n \Delta t$ é um ponto marcador $\left(x_{i}^{n}, y_{i}^{n}\right)$ sobre a frente. O objetivo é obter um algoritmo numérico que produza valores novos $\left(x_{i}^{n+1}, y_{i}^{n+1}\right)$ em relação às posições anteriores.

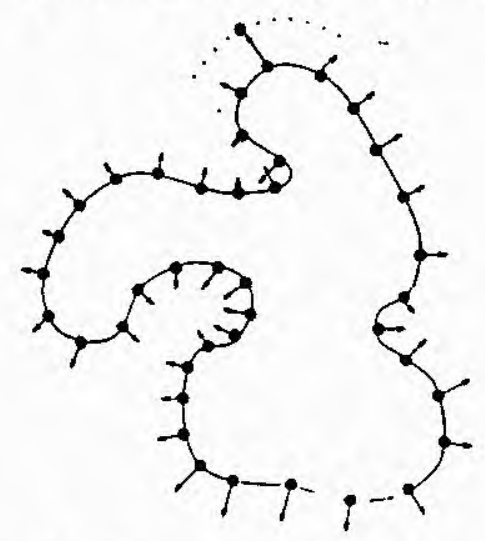

Figura 2.4.1. Parametrização discreta da curva.

Aproximações de diferenças centradas baseadas na série de Taylor resultam em:

$$
\begin{aligned}
\frac{d x_{i}^{n}}{d s}=\frac{x_{i+1}^{n}-x_{i-1}^{n}}{2 \Delta s}, & \frac{d y_{i}^{n}}{d s}=\frac{y_{i+1}^{n}-y_{i-1}^{n}}{2 \Delta s} \\
\frac{d^{2} x_{i}^{n}}{d s^{2}}=\frac{x_{i+1}^{n}-2 x_{i}^{n}+x_{i-1}^{n}}{\Delta s^{2}}, & \frac{d^{2} y_{i}^{n}}{d s^{2}}=\frac{y_{i+1}^{n}-2 y_{i}^{n}+y_{i-1}^{n}}{\Delta s^{2}} .
\end{aligned}
$$

Similarmente, derivadas no tempo podem ser aproximadas por diferenças progressivas.

$$
\frac{d x_{i}^{n}}{d t}=\frac{x_{i}^{n+1}-x_{i}^{n}}{\Delta t}, \quad \frac{d y_{i}^{n}}{d t}=\frac{y_{i}^{n+1}-y_{i}^{n}}{\Delta t}
$$


A substituição dessas aproximações nas equações de movimento dadas por (2.2) produz o esquema

$$
\left(x_{i}^{n+1}, y_{i}^{n+1}\right)=\left(x_{i}^{n}, y_{i}^{n}\right)+\Delta t F\left(\kappa_{i}^{n}\right) \frac{\left(y_{i+1}^{n}-y_{i-1}^{n},-\left(x_{i+1}^{n}-x_{i-1}^{n}\right)\right)}{\left(\left(x_{i+1}^{n}-x_{i-1}^{n}\right)^{2}+\left(y_{i+1}^{n}-y_{i-1}^{n}\right)^{2}\right)^{\frac{1}{2}}},
$$

em que

$$
\kappa_{i}^{n}=4 \frac{\left(y_{i+1}^{n}-2 y_{i}^{n}+y_{i-1}^{n}\right)\left(x_{i+1}^{n}-x_{i-1}^{n}\right)-\left(x_{i+1}^{n}-2 x_{i}^{n}+x_{i-1}^{n}\right)\left(y_{i+1}^{n}-y_{i-1}^{n}\right)}{\left(\left(x_{i+1}^{n}-x_{i-1}^{n}\right)^{2}+\left(y_{i+1}^{n}-y_{i-1}^{n}\right)^{2}\right)^{\frac{3}{2}}} .
$$

Usando o fato de que a curva é fechada, as equações acima permitem calcular as posições das partículas em um nível de tempo posterior.

Quando as partículas marcadoras estão muito próximas o quociente do lado direito da equação (2.22) aproxima-se de $\frac{0}{0}$ causando uma indeterminação. A curvatura calculada pode mudar drásticamente dessas partículas para as próximas devido aos pequenos e inevitáveis erros nas posiçòes, resultando em uma singularidade na frente.

1) Caso Suave: Podemos verificar essa instabilidade usando um esquema de partículas marcadoras para evoluir a curva inicial cosseno propagando com velocidade $F(\kappa)=1$ $\epsilon \kappa, \epsilon=0.25$. Para $\epsilon>0$, a solução exata é sempre suave e diferenciável.

Foram utilizados 50 pontos marcadores para $\Delta t=0.001, \Delta t=0.0001, \Delta t=0.00001$. Para $\Delta t=0.001$, por exemplo, a frente ao propagar-se começa a criar cantos (ver Figura 2.4.2), oscilaçōes logo desenvolvem-se e crescem, incontrolavelmente. Essas oscilações resultam em um ciclo de relações: (1) erros cometidos na discretização das posições marcadoras produzem (2) variações locais nas derivadas calculadas, causando (3) variações nas velocidades das partículas calculadas, que causam (4) avanços irregulares de marcadores produzindo (5) erros maiores na aproximação das posições marcadoras.

Para tentar aumentar a precisão foram usados $\Delta t=0.0001$ e $\Delta t=0.00001$. Com $\Delta t=0.0001$, novamente a solução tornou-se instável, e a continuação suave da solução não foi observado. Somente para $\Delta t=0.00001$ a solução foi aceitável, isto é, não apareceram singularidades. (veja Figura 2.4.3. (a) e (b))

Para qualquer $\epsilon>0$ existe um limite mínimo na distância entre duas partículas, e assim um pequeno passo temporal deve ser utilizado para assegurar estabilidade. Por exemplo, para $\epsilon=0.1$, foi necessário $\Delta t=0.000005$. Para valores de $\epsilon$ suficientemente pequenos, esta aproximação torna-se impraticável.

O que pode ser feito? Três estratégias tentam amenizar esse efeito: 
- Suavizar a função velocidade, a fim de que os pontos marcadores permaneçam longe o bastante para permitir um passo temporal razoável.

- Redistribuir as partículas marcadoras de acordo com o comprimento de arco.

- Produzir alguma técnica de filtragem para remover oscilações nas posições da partículas quando elas evoluem.

Embora essas três técnicas sejam usadas na prática, elas partilham o mesmo princípio. Alteram as equações de movimento de maneiras não óbvias e podem exigir quantidades significantes de suavização para assegurar um $\Delta t$ prático. Assim, elas prejudicam as características mais importantes de propagação, tais como, frentes aguçando e singularidades na curvatura, o que não é desejável, pois queremos representar o movimento de frentes o mais fielmente possível.

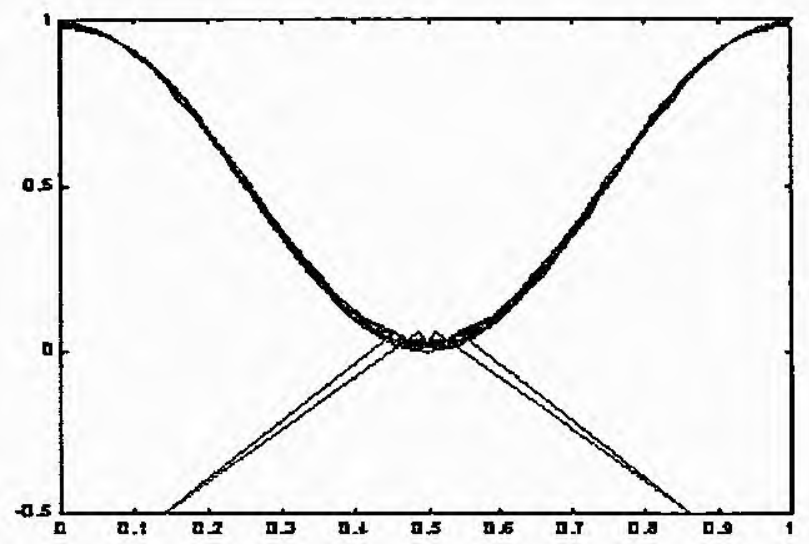

Figura 2.4.2. (a) $\Delta t=0.001, \quad F(\kappa)=1-0.25 \kappa$.
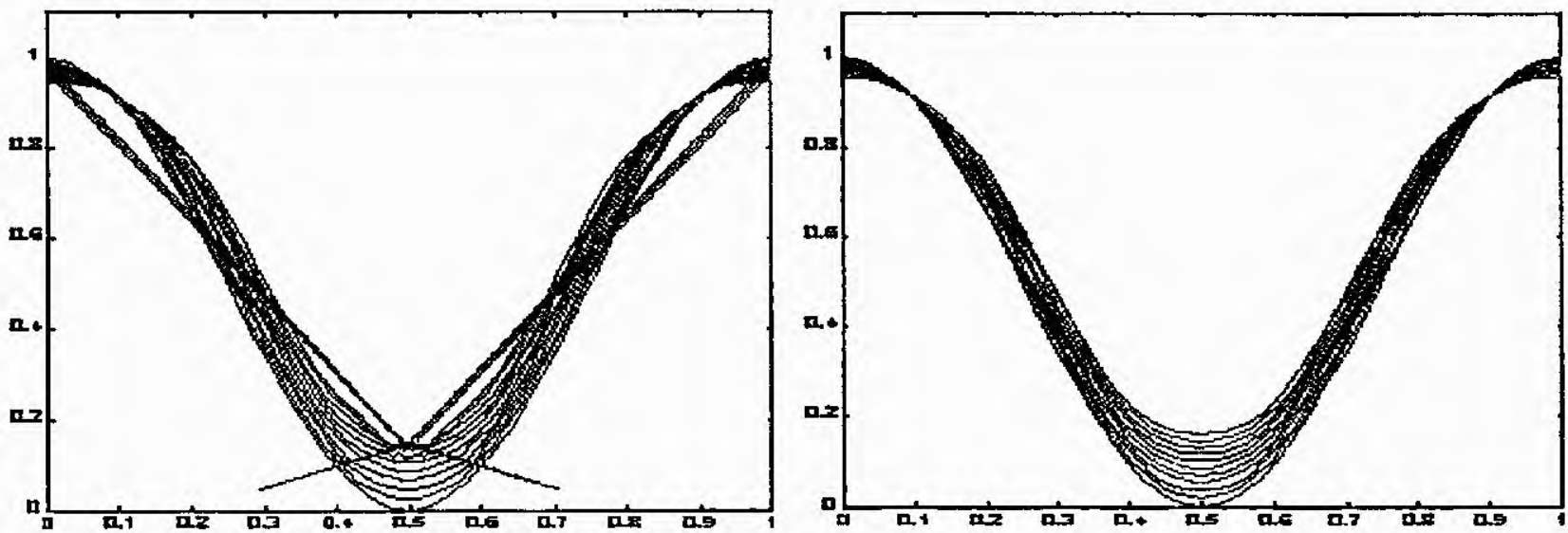

Figura 2.4.3. (a) $\Delta t=0.0001, F=1-0.25 \kappa \quad$ (b) $\Delta t=0.00001, F=1-0.25 \kappa$.

Para $\epsilon=0$, a solução desenvolve cantos e uma condição de entropia deve ser invocada para produzir uma solução fraca fora da formação de singularidades.

Resumindo, o método das partículas marcadoras gera esquemas baseados em uma descrição paramétrica da frente em movimento. Esse método pode ser preciso para pequenos 
movimentos de interfaces, mas em geral, pode apresentar instabilidades e mudanças na topologia da frente.

2) Caso Não Suave: Seja $F(\kappa)=1$ c considere o problema de valor inicial dado por

$$
\begin{gathered}
\psi_{t}=F\left(1+\psi_{x}^{2}\right)^{1 / 2} \\
\psi(x, 0)=f(x)= \begin{cases}1 / 2-x & \text { se } x \leq 1 / 2 \\
x-1 / 2 & \text { se } x>1 / 2\end{cases}
\end{gathered}
$$

A frente inicial é um "V" formado por retas que se interseptam em $(1 / 2,0)$. Utilizando o princípio de construção de Huygens, a solução entrópica em qualquer tempo $t$ é o conjunto de todos os pontos localizado uma distância $t$ da frente inicial "V". Para construir um esquema numérico de diferenças, dividimos o intervalo $[0,1]$ em $2 M-1$ pontos e discretizamos $(2.24)$ pelas aproximações já citadas, e assim temos

$$
\psi_{t} \approx \frac{\psi_{i}^{n+1}-\psi_{i}^{n}}{\Delta t}=\left[1+\left[\frac{\psi_{i+1}^{n}-\psi_{i-1}^{n}}{2 \Delta x}\right]^{2}\right]^{1 / 2}
$$

Quando $x_{M}=1 / 2$, pela simetria temos que $\psi_{M+1}=\psi_{M-1}$; assim o lado direito de (2.26) é igual a 1 independentemente dos passos $\Delta x$ e $\Delta t$. Entretanto, para todo $x \neq$ $1 / 2, \psi_{t}$ será igual a $\sqrt{2}$, devido ao gráfico ser linear sobre ambos os lados do canto, e portanto a aproximação de diferenças centrais é exata. Não importa quão pequeno fazemos os parâmetros numéricos, $\Delta x$ e $\Delta t$, contanto qué usamos um número ímpar de pontos, a aproximação para $\psi_{t}$ em $x=1 / 2$ não melhora. Isto acontece devido à maneira com que a derivada, $\psi_{x}$, foi aproximada. A Figura 2.4.4 mostra o resultado numérico deste esquema.

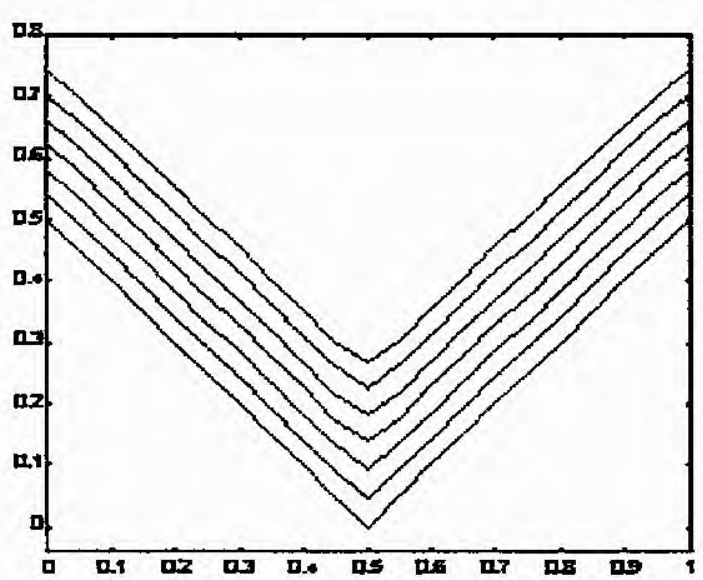

Diferenças centrais $\Delta t=0.005$

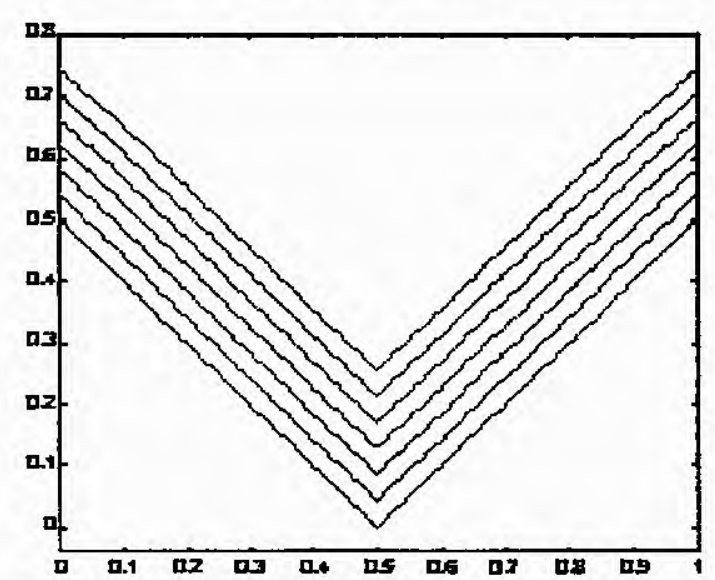

Diferenças centrais $\Delta t=0.0005$.

Figura 2.4.4. 
Infelizmente, a aproximação de diferenças centrais escolhe uma diferente (e, para nosso propósito, errada) solução limite. Ela coloca a inclinação $\psi_{x}$ igual à média das inclinações à csquerda e direita. Quando o cálculo avança, este erro de cálculo das inclinaçōes propaga instabilidades e consequentemente surgem oscilações drásticas. Para evitar esse efeito, desenvolveremos esquemas com propriedades especiais para aproximar o termo $|\nabla \phi|$, a fim de que tratem corretamente os cantos (choques) existentes na frente. Isso é o que veremos no Capítulo 3. 


\section{Capítulo 3}

\section{Teoria e Esquemas Numéricos para Equações Hiperbólicas}

\subsection{Introdução}

Neste capítulo fazemos uma revisão dos aspectos mais relevantes da teoria de leis de conservação hiperbólicas com o intuito de desenvolver esquemas para resolver numericamente uma equação diferencial parcial na forma conservativa. O objetivo principal foi o de encontrar esquemas simples, estáveis e precisos que sejam adequados para o tratamento de choques e descontinuidades. Esses esquemas poderão ser utilizados para aproximar as leis de conservação hiperbólicas obtidas das formulações de valor de fronteira e inicial que modelam o movimento de frentes. Dentre os esquemas nos quais nos concentramos podemos citar os do tipo Upwind, Monótonos, Entropia, TVD, Limitantes de Fluxo e Limitantes de Inclinação. Nem todos são usados para aproximar ambas as formulações, conforme veremos no Capítulo 4, mas foram estudados com o objetivo de aumentar nossos conhecimentos em esquemas numéricos para leis de conservação.

Na Seção 3.2 deste capítulo, as referências básicas utilizadas foram as seguintes: [15], [26], [41], [43, 44], enquanto que, na Seção 3.3, utilizamos: [12], [15], [17], [19, 20, 21], [25], $[26],[29],[42],[43,44]$. 


\subsection{Breve Introdução à Teoria de Equações Hiperbólicas}

A equação hiperbólica mais simples é a equação linear de advecção, também conhecida como equação da onda linear. a saber

$$
v_{t}(x, t)+a v_{x}(x, t)=0
$$

O problema de valor inicial, ou problema de Cauchy para esta equação consiste em encontrar uma função $v(x, t)$ no semi-plano $-\infty<x<+\infty, \quad t \geq 0$, que satisfaça (3.1) juntamente com a condição inicial

$$
v(x, 0)=f(x)
$$

Quando o tempo avança, o dado inicial propaga-se para a direita (se $a>0$ ) ou para a esquerda (se $a<0$ ) com velocidade $a$. A solução para esse problema de valor inicial é dada por $v(x, t)=f(x-a t), \quad t \geq 0$ e é constante sobre retas de inclinação $1 / a$ no plano- $(x, t)$, que são conhecidas como características da equação.

Notemos que a curvas características dadas por $x-a t=x_{0}$ satisfazem as equações diferenciais ordinárias $x^{\prime}(t)=a, \quad x(0)=x_{0}$. Se diferenciarmos $v(x, t)$ ao longo de uma dessas características, temos

$$
\frac{d v(x(t), t)}{d t}=\frac{\partial v(x(t), t)}{\partial x} \frac{d x}{d t}+\frac{\partial v(x(t), t)}{\partial t}=x^{\prime}(t) v_{x}+v_{t}=v_{t}+a v_{x}=0
$$

confirmando que $v$ é constante ao longo das linhas características.

Considere um ponto $A$ localizado no plano- $(x, t)$ (veja Figura 3.2.1). Como vimos que $v$ é constante ao longo das características, a solução no ponto $A$ pode ser encontrada traçando uma reta pelo ponto $A$ com inclinaçào $1 / a$, encontrando o eixo $x$ no ponto $B$. Já que a solução é constante ao longo dessa reta, temos que o valor da solução em $A$ será o mesmo que o da condição inicial em $B$. Assim a solução em $A$ depende do valor em $B$ e portanto dizemos que o domínio de dependência do ponto $A$ é o ponto $B$. Inversamente, o domínio de influência do ponto $B$ é o conjunto de todos os pontos (incluindo $A$ ) sobre a reta com inclinação $1 / a$ saindo de $B$. A Figura 3.2 .1 para $a=1$. mostra as características da equação $v_{t}=-v_{x}$ 


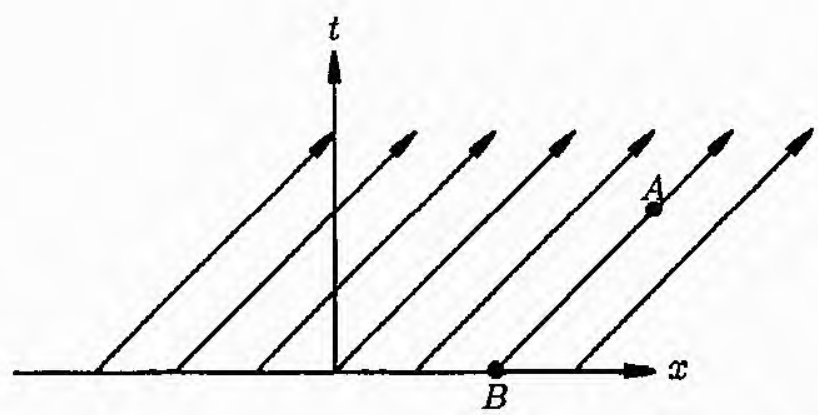

Figura 3.2.1. Domínio de dependência e influência.

As análises dos domínios de dependência e de influência para uma equação hiperbólica serão importantes pois reforçam o papel fundamental das características na construção da solução. Para equações hiperbólicas mais gerais, as características não necessariamente serão linhas retas e paralelas, mas o mesmos conceitos se aplicam como veremos a seguir.

\subsubsection{Propagação de Singularidades e Choques}

A situação no caso não linear, tanto no que se refere à propagação de singularidades quanto ao comportamento da solução, é bastante diferente do caso linear. Para se ter uma idéia do que pode acontecer, vamos considerar a equação da onda não linear com velocidade variável $a(x, t, v)$,

$$
v_{t}(x, t)+a(x, t, v) v_{x}(x, t)=0
$$

Vimos que se $a$ é uma função constante, então as características são retas paralelas. O fato mais importante para o caso não linear a ser analisado é que as características podem se interceptar ocasionando singularidades na soluçāo. Quando duas ou mais características se intersectam em um ponto, essa colisão recebe o nome de choque. Para simplificar a apresentação dos conceitos que precisamos, vamos considerar a equação não linear de Burgers, dada por:

$$
v_{t}(x, t)+v(x, t) v_{x}(x, t)=0
$$

onde a velocidade de propagação é nesse caso $a(v)=v(x, t)$. Considere uma partícula movendo-se no plano- $(x, t)$ cuja posição $x$ em qualquer tempo $t$ é parametrizada por $s$. Pela regra da cadeia.

$$
\frac{d v(x(s), t(s))}{d s}=\frac{\partial v(x, t)}{\partial x} \frac{d x}{d s}+\frac{\partial v(x, t)}{\partial t} \frac{d t}{d s}=\frac{d t}{d s}\left[v_{t}+\frac{d x}{d t} v_{x}\right]
$$


Agora, suponhamos que a trajetória da partícula é a curva tal que $\frac{d x}{d t}=v$. Utilizando a equação (3.5) então $\frac{d v}{d s}=0$. Assim, $v$ é constante ao longo das características, que são as curvas definidas por $\frac{d x}{d t}=v$ e que ncssc caso serão retas, pois lembre-se que $v$ é constante ao longo das características de forma que $\frac{d x}{d t}=c t e$. Note no entanto que agora as retas podem não ser paralelas, pois a constante de uma delas pode ser diferente da de outra, conforme veremos no exemplo a seguir.

Considere a equação não linear de Burgers (3.5) e a condição inicial

$$
v(x, 0)= \begin{cases}1, & \text { se } x<0 \\ 1-x, & \text { se } 0 \leq x \leq 1 \\ 0, & \text { se } x>1\end{cases}
$$

Nesse exemplo, as características são retas como mostrado na Figura 3.2 .2 e colidem para $t \geq 1$. Cada uma sai da condição inicial com inclinação $1 / v$, onde $v$ é dado por (3.7) e transportam os valores da condição inicial para dentro do domínio.

As equações dessas características passando pelo ponto $\left(x_{0}, 0\right)$ são:

$$
x= \begin{cases}t+x_{0}, & \text { se } x_{0}<0 \\ \left(1-x_{0}\right) t+x_{0}, & \text { se } 0 \leq x_{0} \leq 1 \\ x_{0}, & \text { se } x_{0}>1\end{cases}
$$

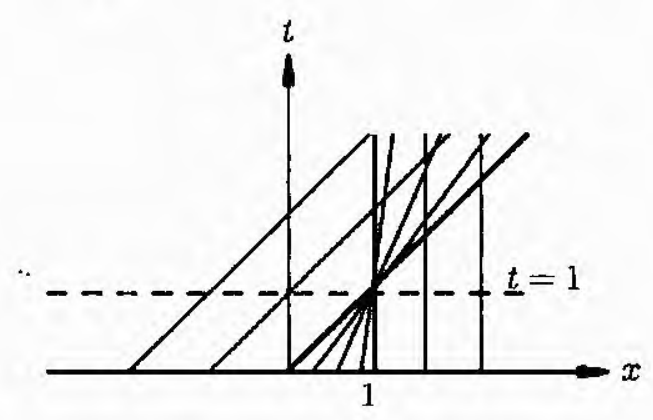

Figura 3.2.2. Características para o problema (3.7)

A solução desse problema, definida para $0 \leq t<1$, é:

$$
v(x, t)= \begin{cases}1, & \text { se } x<t<1 \\ \frac{1-x}{1-t}, & \text { se } t \leq x<1 \\ 0, & \text { se } t<1 \leq x\end{cases}
$$

Não existe uma soluçāo global, isto é, definida para todo $t>0$, que seja contínua no ponto $(1,1)$, uma vez que $v \equiv 1$ na região $x<t<1$ e $v \equiv 0$ na região $t<1 \leq x$; além 
disso, mesmo admitindo soluções descontínuas (por exemplo, funções que são descontínuas ao longo de uma curva suave e que satisfazem a EDP fora dessa curva), não saberíamos como determinar a solução na região $1<x<t$ uma vez que cada ponto nessa região está em exatamente três características (veja Figura 3.2.2).

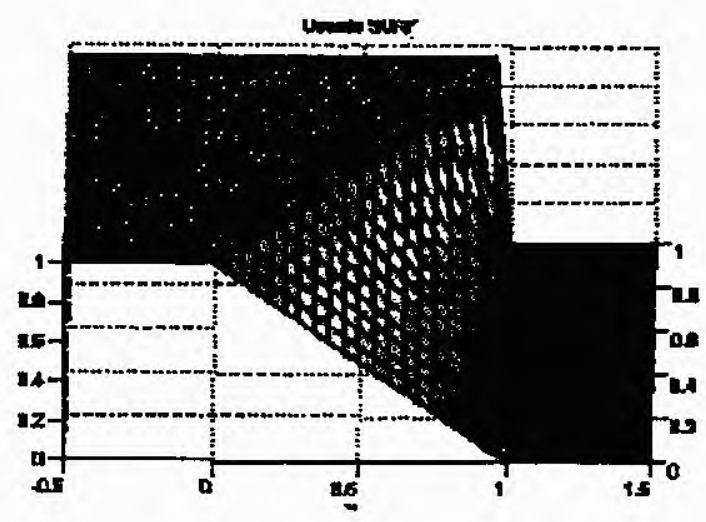

Figura 3.2.3. Gráfico de $v(x, t)$

Conforme mostrado pelo gráfico da Figura 3.2.3, não há regularidade na solução devido à interseção das características e por isso é preciso entender a solução em um sentido local e mais fraco. Assim, um critério para a escolha da solução fraca é admitir que esta seja o caso limite das soluções de problemas constituídos da mesma equação e de aproximações contínuas dos dados iniciais (não trataremos aqui, veja $[11,26]$ ) ou o caso limite da solução da regularização da própria equação, que se dá pela adição de um termo viscoso à equação original transformando-a em uma equação parabólica como já comentado no Capítulo 1, da forma,

$$
v_{t}(x, t)+v(x, t) v_{x}(x, t)=\epsilon v_{x x}(x, t)
$$

Como no Capítulo 1, a solução limite será a solução entrópica.

\subsubsection{Função de Fluxo, Condições de Entropia e Soluções Fracas}

Considere a equaçāo hiperbólica não linear escrita na forma conservativa, ou lei de conservação

$$
v_{t}+[G(v)]_{x}=0
$$

Em (3.11), a função $G$ é denominada função de fluxo. Note que se a função de fluxo $G(v)=v$, então $v_{t}+v_{x}=0$ (caso linear), e se $G(v)=v^{2} / 2$, então $v_{t}+v v_{x}=0$ (equaçäo 
não linear de Burgers). A seguir, mostramos que a quantidade $v$ é conservada ao longo do tempo no volume unidimensional $[\bar{a}, b]$. Com esse objetivo, integramos ambos os lados da equação (3.11) obtendo,

$$
0=\int_{a}^{b}\left(v_{t}+[G(v)]_{x}\right) d x=\int_{a}^{b} v_{t} d x+\int_{a}^{b}[G(v)]_{x} d x=\frac{d}{d t} \int_{a}^{b} v d x+G(v(b, t))-G(v(a, t))
$$

Assim

$$
\frac{d}{d t} \int_{a}^{b} v d x=G(v(a, t))-G(v(b, t))
$$

Esta equação fornece uma interpretação física da Lei de Conservação Hiperbólica dada por (3.11). A variação na quantidade $v$ entre $a$ e $b$ é igual ao fluxo dessa quantidade $G(v)$ fluindo nesse intervalo, por isso $G$ é chamada de função de fluxo (veja Figura 3.2.4).

Dizemos que $v$ é conservado em (3.13) porque existe um balanceamento entre a mudança de $v$ no intervalo $[a, b]$ e o fluxo do material no intervalo. Observe que a forma integrada (3.13) não exige que $v$ seja diferenciável com respeito a $x$, assim a classe das soluções pode ser consideravelmente ampliada incluindo soluções descontínuas.

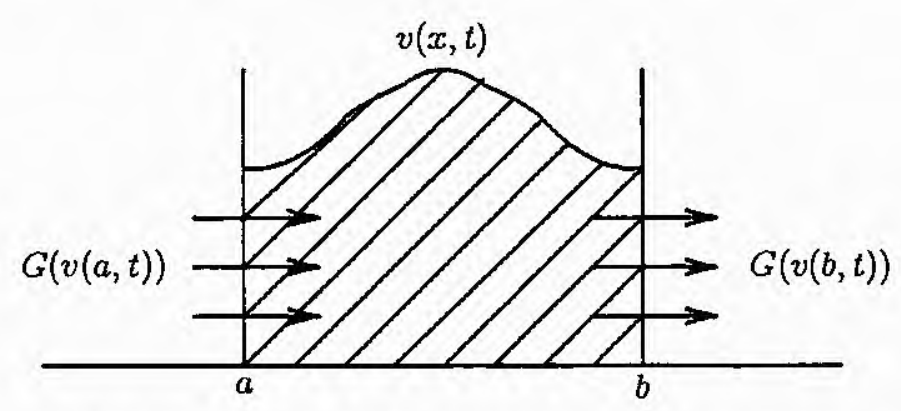

Figura 3.2.4. Fluxo $G$ da quantidade $v$ no intervalo $[a, b]$.

Nesta seção estabelecemos precisamente o que entendemos por uma solução fraca. Com esse objetivo, definimos o conjunto de funções testes, $C_{0}^{1}$, como sendo o conjunto

$$
\left\{\phi \in C^{1}:\{(x, t) \in \mathbb{R} \times[0, \infty): \phi(x, t) \neq 0\} \subset[a, b] \times[0, T] \text { para algum } a, b \text { e } T\right\} .
$$

A função $\phi$ é continuamente diferenciável (classe $C^{1}$ ), e nula fora de algum retângulo no plano- $(x, t)$. As funções em $C_{0}^{1}$ são ditas ter suporte compacto em $\mathbb{R} \times[0, \infty)$. O suporte de $\phi$, denotado por $\operatorname{supp}(\phi)$, é o conjunto sobre o qual $\phi(x, t) \neq 0$. 
Seja o operador $\nabla \equiv\left(\partial_{t}, \partial_{x}\right)$ e o vetor $w_{v}=(v, G(v))$. Então (3.11) pode ser escrita na forma

$$
\nabla \cdot w_{v}=0
$$

Se multiplicarmos a equação acima por $\phi \in C_{0}^{1}$, então, $\phi \nabla \cdot w_{v}=0$. Integrando essa última equação sobre $-\infty<x<\infty$ e $t \geq 0$, temos

$$
\int_{0}^{+\infty} \int_{-\infty}^{+\infty} \phi \nabla \cdot w_{v} d x d t=0
$$

Usando integração por partes e o fato de que $\phi \in C_{0}^{1}$, ou seja $\phi(x, t)=0$ fora do retângulo $[a, b] \times[0, T]$, obtemos

$$
\begin{aligned}
0 & =\int_{0}^{+\infty} \int_{-\infty}^{+\infty} \phi \nabla \cdot w_{v} d x d t=\int_{0}^{+\infty} \int_{-\infty}^{+\infty}\left[v_{t}+G(v)_{x}\right] \phi(x, t) d x d t \\
& \left.=\int_{0}^{T} \int_{a}^{b}\left[v_{t}+G(v)_{x}\right] \phi(x, t) d x d t \quad \text { (devido } \phi \in C_{0}^{1}\right) \\
& =\int_{a}^{b} \int_{0}^{T} v_{t} \phi(x, t) d t d x+\int_{0}^{T} \int_{a}^{b} G(v)_{x} \phi(x, t) d x d t \\
& =\int_{a}^{b}\left\{[v \phi]_{t=0}^{t=T}-\int_{0}^{T} v \phi_{t} d t\right\} d x+\int_{0}^{T}\left\{[G(v) \phi]_{x=a}^{x=b}-\int_{a}^{b} G(v) \phi_{x} d x\right\} d t \\
& =-\int_{a}^{b} v(x, 0) \phi(x, 0) d x-\int_{a}^{b} \int_{0}^{T} v \phi_{t} d t d x-\int_{0}^{T} \int_{a}^{b} G(v) \phi_{x} d x d t
\end{aligned}
$$

pois $\phi(x, T)=\phi(a, t)=\phi(b, t)=0$. Podemos reescrever (3.14)-(3.15) como

$$
\int_{0}^{+\infty} \int_{-\infty}^{+\infty}\left[v \phi_{t}+G(v) \phi_{x}\right] d x d t+\int_{-\infty}^{+\infty} f(x) \phi_{0} d x=0
$$

em que $f(x)=v(x, 0)$ é a condição inicial e $\phi_{0}$ é uma notação para $\phi(x, 0)$. Notamos que, como o suporte de $\phi$ está contido em $[a, b] \times[0, T]$ e $\phi$ está definida em $\mathbb{R} \times[0, \infty), \phi(x, 0)$ não precisa ser zero.

Portanto, o que apresentamos acima é a demonstração da seguinte proposição.

Proposição 3.2.1. Se v é uma solução clássica do problema de valor inicial

$$
\begin{aligned}
v_{t}+\frac{\partial}{\partial x} G(v) & =0 \\
f(x) & =v(x, 0), \quad x \in \mathbb{R},
\end{aligned}
$$

então $v$ satisfaz a equação (3.16) para todo $\phi \in C_{0}^{1}$. 
A seguinte proposiçāo diz que se $v$ é suave a recíproca é válida, ou seja, (3.16) implica em $\nabla . w_{v}=0$ para toda $\phi \in C_{0}^{1}$.

Proposiçāo 3.2.2. Se v é de classe $C^{1}$ em relação a x.t. e satisfaz a equação (3.16) para toda $\phi \in C_{0}^{1}$, então $v$ é uma soluçâo clássica do problema de valor inicial (3.17)-(3.18).

Devemos ter em mente que podem existir algumas soluções para a equação (3.16) que nào são soluçòes clássicas do problema de valor inicial (3.17)-(3.18). ou seja. funções que satisfazem (3.16) mas que podem não ser diferenciáveis. Por esta razão temos a seguinte definiçào.

Definição 3.2.3. Se v satisfaz a equação (3.16) para toda $\phi \in C_{0}^{1}$, então v é dita ser uma solução fraca do problema de valor inicial (3.17)-(3.18).

Considere uma solução fraca $w_{v}$ de (3.16), descontínua ao longo de alguma curva suave $L$ cuja equaçào é $x=\psi(t)$ no plano- $(x, t)$ (veja. Figura 3.2.5).

A região $\underset{\sim}{D}$. no semi-plano superior $\left(\mathbb{R} \times \mathbb{R}^{+}\right)$, pode ser dividida em duas regiòes $D_{\sim}$ e $D_{R}$ pela curva $L$. Desde que $w_{v}$ é uma solução fraca de (3.16).

$$
\iint_{D}(\nabla \phi) \cdot w_{v} d x d t=\iint_{D_{D_{L}}}(\nabla \phi) \cdot w_{v} d x d t+\iint_{D_{\sim}}(\nabla \phi) \cdot w_{v} d x d t=0
$$

em que $\phi$ é qualquer função suave, que se anula sobre toda a fronteira de $\underset{\sim}{D}$. Considerando a segunda integral à esquerda de (3.19). podemos escrevè-la como

$$
\iint_{D_{\mathcal{L}}}(\nabla \phi) \cdot w_{v} d x d t=\iint_{D_{\mathcal{L}}} \nabla \cdot\left(\phi w_{v}\right) d x d t-\iint_{D_{\sim}} \phi\left(\nabla \cdot w_{v}\right) d x d t
$$

Aplicando o teorema da Divergência para a primeira integral a direita da igualdade. temos

$$
\iint_{D_{\mathcal{L}}}(\nabla \phi) \cdot w_{v} d x d t=\int_{L_{L}} \dot{\phi} w_{v} \cdot \vec{n} d s-\iint_{D_{\sim}} \phi\left(\nabla \cdot w_{v}\right) d x d t
$$

em que $\vec{n}$ é o vetor normal a $L$ com orientação representada como na Figura 3.2.5. Se a solução $w_{v}$ é suave em $D_{L}$, então $\nabla \cdot w_{v}=0 \mathrm{e}$

$$
\iint_{D_{L}} \phi\left(\nabla \cdot w_{v}\right) d x d t=0
$$


Portanto,

$$
\iint_{D_{D_{L}}}(\nabla \phi) \cdot w_{v} d x d t=\int_{L_{L}} \phi w_{v} \cdot \vec{n} d s
$$

Similarmente, podemos repetir para a segunda integral do lado direito de (3.19), e assim temos

$$
\iint_{D_{R}}(\nabla \phi) \cdot w_{v} d x d t=-\int_{L_{R}} \phi w_{v} \cdot \vec{n} d s
$$

em que o sinal em (3.21) resulta da orientação do vetor normal unitário $\vec{n}$ (veja Figura 3.2.5).

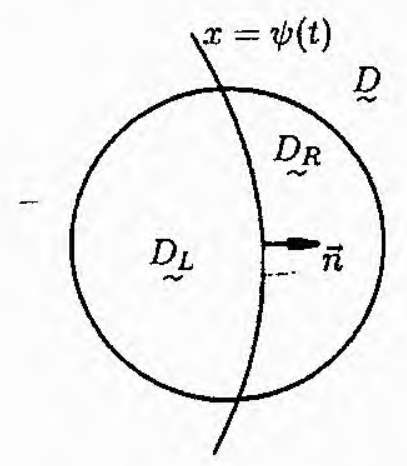

Figura 3.2.5. .

Com a substituição de (3.20) e (3.21) em (3.19), obtemos a condição que $w_{v}$ deve satisfazer ao longo da descontinuidade $L$,

$$
\int_{L_{L}} \phi w_{v} \cdot \vec{n} d s-\int_{L_{R}} \phi w_{v} \cdot \vec{n} d s=0 .
$$

Como esse resultado é válido para qualquer função $\phi$, devemos ter,

$$
\left(\left.w_{v}\right|_{L}-\left.w_{v}\right|_{R}\right) \cdot \vec{n}=0
$$

sobre $L$. Os símbolos $\left.w_{v}\right|_{L}$ e $\left.w_{v}\right|_{R}$ denotam

$$
\begin{array}{ll}
\left.w_{v}\right|_{L}=\lim _{(x, t) \rightarrow L} w_{v}, & (x, t) \in D_{\sim}, \\
\left.w_{v}\right|_{R}=\lim _{(x, t) \rightarrow L} w_{v}, & (x, t) \in D_{\sim} .
\end{array}
$$

Seja [ ] o salto sobre a descontinuidade, por exemplo, $[f(v)]=f\left(v_{L}\right)-f\left(v_{R}\right)$. Com isto, (3.22) torna-se

$$
\left[w_{v} \cdot \vec{n}\right]=0
$$


conhecida como a condição de Rankine-Hugoniot ou condição de salto. Se $S$ denota a velocidade de propagaçāo da descontinuidade, então $S=\frac{d \psi}{d t}$. Da equação da curva $L, \psi(t)-x=0$, temos que 0 vetor normal $\vec{n}$ possui componentes $\vec{n}=\left(\frac{d \psi}{d t},-1\right)=(S,-1)$. Então a condição de R-H dada por (3.23), torna-se

$$
S=\frac{G\left(v_{L}\right)-G\left(v_{R}\right)}{v_{L}-v_{R}},
$$

em que $v_{L}$ e $v_{R}$ são os estados à esquerda e à direita da descontinuidade.

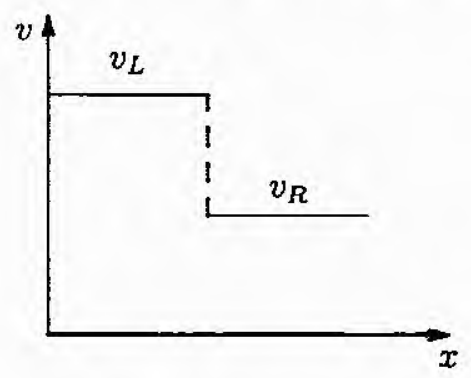

Figura 3.2.6. Perfil de $v$ em um dado tempo.

O comportamento da função de fluxo $G$ possui consequências profundas sobre o comportamento da soluçào $v(x, t)$ da lei de conservação. Uma propriedade importante é a monotonicidade da velocidade da característica $\lambda(v)=G^{\prime}(v)$. Existem essencialmente três possibilidades:

- $\lambda(v)$ é uma função monótona crescente de $v$, i.e, $\frac{d \lambda}{d v}=\lambda^{\prime}(v)=G^{\prime \prime}(v)>0$ (fluxo convexo)

- $\lambda(v)$ é uma função monótona decrescente de $v$, i.e, $\frac{d \lambda}{d v}=\lambda^{\prime}(v)=G^{\prime \prime}(v)<0$ (fluxo côncavo) - $\lambda(v)$ possui extremo, para algum $v$, i.e, $\lambda^{\prime}(v)=G^{\prime \prime}(v)=0$ (fluxo não convexo, não côncavo)

A fim de ilustrar os conceitos de solução, discutidos acima. iremos exibir em forma de exemplo várias soluçōes fracas para a equação de Burgers,

$$
v_{t}+[G(v)]_{x}=0, \quad G(v)=\frac{v^{2}}{2}
$$

Exemplo 3.2.4. A função

$$
v(x, t)= \begin{cases}1, & \text { se } x \leq t / 2 \\ 0, & \text { se } x>t / 2 .\end{cases}
$$

é uma soluçâo fraca para a equação de Burgers com condição inicial

$$
v(x, 0)= \begin{cases}1, & \text { se } x \leq 0 \\ 0, & \text { se } x>0\end{cases}
$$


Demonstração: Seja $\phi \in C_{0}^{1}$ e $a, b$ e $T$ tal que $\operatorname{supp}(\phi) \subset[a, b] \times[0, T]$. Então

$\int_{0}^{+\infty} \int_{-\infty}^{+\infty}\left(v \phi_{t}+\frac{v^{2}}{2} \phi_{x}\right) d x d t+\int_{-\infty}^{+\infty} v(x, 0) \phi(x, 0) d x$

$=\int_{0}^{T} \int_{a}^{b}\left(v \phi_{t}+\frac{v^{2}}{2} \phi_{x}\right) d x d t+\int_{a}^{b} v(x, 0) \phi(x, 0) d x=\int_{0}^{T} \int_{a}^{t / 2}\left(\phi_{t}+\frac{1}{2} \phi_{x}\right) d x d t+\int_{a}^{0} \phi(x, 0) d x$

$=\int_{a}^{0} \int_{0}^{T} \phi_{t} d t d x+\int_{0}^{T / 2} \int_{2 x}^{T} \phi_{t} d t d x+\frac{1}{2} \int_{0}^{T} \int_{a}^{t / 2} \phi_{x} d x d t+\int_{a}^{0} \phi(x, 0) d x$

$=\int_{a}^{0}[\phi(x, T)-\phi(x, 0)] d x+\int_{0}^{T / 2}[\phi(x, T)-\phi(x, 2 x)] d x+\frac{1}{2} \int_{0}^{T}[\phi(t / 2, t)-\phi(a, t)] d t+\int_{a}^{0} \phi(x, 0) d x$

$=-\frac{1}{2} \int_{0}^{T} \phi(y / 2, y) d y+\frac{1}{2} \int_{0}^{T} \phi(t / 2, t) d t=0$.

$\mathrm{Na}$ demonstração acima, utiliza-se o fato que $\phi(x, T)=\phi(a, t)=0$ e assim eliminam-se algumas integrais. Também faz-se a mudança de variável $y=2 x$ para cancelar outras. As demais demonstrações são feitas de maneira análoga.

Observação 3.2.5. Uma descontinuidade de uma solução fraca contínua por partes é um choque se as características de ambos os lados da descontinuidade intersectam a curva de descontinuidade na direção de crescimento de $t$, como é o caso na Figura 3.2.7. Matematicamente, uma descontinuidade é um choque se:

$$
G^{\prime}\left(v_{L}\right)>S>G^{\prime}\left(v_{R}\right)
$$

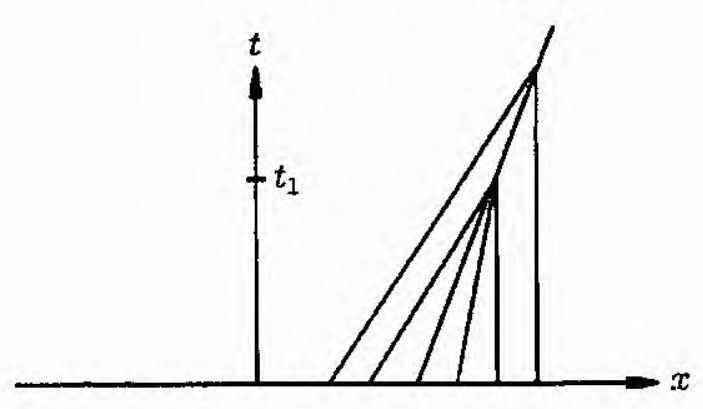

Figura 3.2.7. Características convergindo: formação de choques.

Observação 3.2.6. Note que pela forma da solução (3.26), a descontinuidade na solução propaga ao longo da curva $x=t / 2$. Portanto a velocidade de propagação da descontinuidade é $\frac{d x}{d t}=\frac{1}{2}$. Para o Exemplo 3.2.4, temos que $G^{\prime}(v)=v$. Assim $G^{\prime}\left(v_{L}\right)=1, G^{\prime}\left(v_{R}\right)=0$ e $S=1 / 2$ e portanto a desigualdade (3.28) é verificada, implicando que a descontinuidade é um choque.

Exemplo 3.2.7. A solução

$$
v(x, t)= \begin{cases}0, & \text { se } x \leq t / 2 \\ 1, & \text { se } x>t / 2\end{cases}
$$


é uma solução fraca para a equação de Burgers com condição inicial

$$
v(x, 0)= \begin{cases}0, & \text { se } x \leq 0 \\ 1, & \text { se } x>0\end{cases}
$$

Observação 3.2.8. Analogamente ao Exemplo 3.2.4, a velocidade de propagação da descontinuidade na solução (3.29) é $S=1 / 2$. Note que para este Exemplo $G^{\prime}\left(v_{L}\right)=0$ e $G^{\prime}\left(v_{R}\right)=1$ e portanto (3.28) não é verificada, implicando que a descontinuidade na solução não é um choque e sim uma rarefação.

Exemplo 3.2.9. A função

$$
v(x, t)= \begin{cases}0, & \text { se } x<0 \\ \frac{x}{t}, & \text { se } 0 \leq x<t \\ 1, & \text { se } x>t\end{cases}
$$

também é uma solução fraca para a equação de Burgers com condiçâo inicial (3.30).

A Figura 3.2.8 abaixo mostra parte das características para o Exemplo 3.2.7. As figuras 3.2.9 mostram duas maneiras distintas de preencher a região vazia.

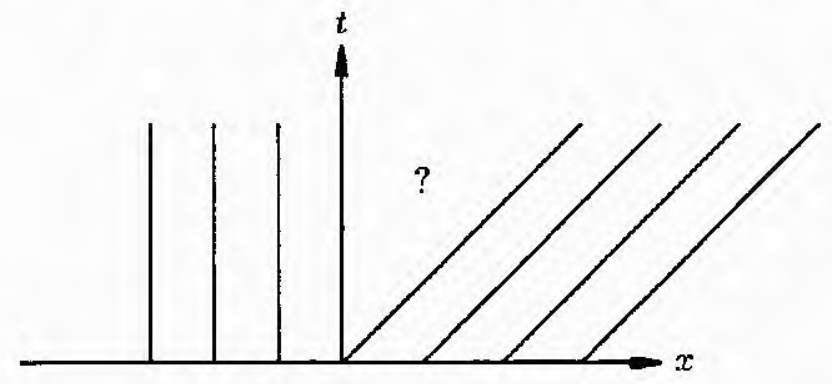

Figura 3.2.8. Características divergindo: falha na solução.

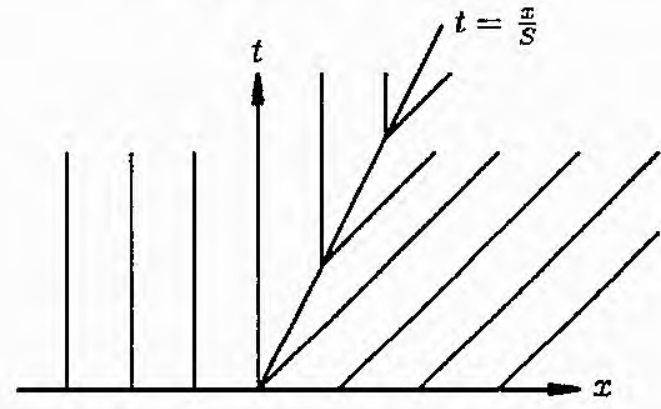

(a) Choque de rarefação

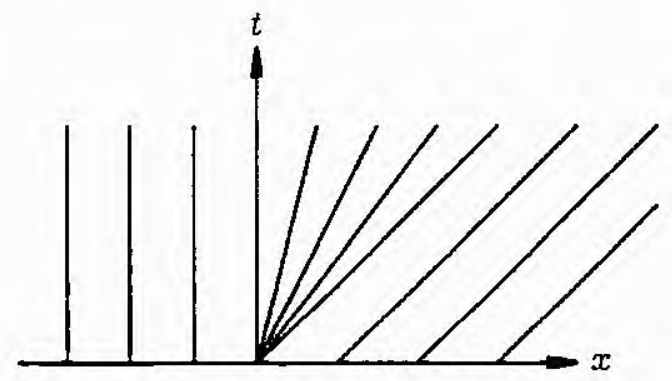

(b) Leque de rarefação

Figura 3.2.9. Curvas características associadas com a solução do problema de Burgers (3.25) para os Exemplos 3.2.7, 3.2.9, respectivamente. 
Observação 3.2.10. Nos Exemplos 3.2.7 e 3.2.9, constatamos que soluções fracas para os mesmos problemas de valor inicial não são únicas. A solução no Exemplo 3.2 .7 corresponde a preencher a região que nâo tem curvas características com características que saem da curva $t=2 x$, como mostrado na Figura 3.2.9(a).

A solução no Exemplo 3.2 .9 corresponde a preencher a região vazia com um leque de características como mostrado na Figura 3.2.9(b). Note que nos é permitido preencher a região vazia no minimo de duas maneiras diferentes que são compatíveis com a formulação fraca do problema. Mais a frente mostraremos que as soluções encontradas preenchendo a região como um leque serão as soluções desejadas.

Exemplo 3.2.11. Consideremos a equação de Burgers (3.25) com condição inicial

$$
v(x, 0)= \begin{cases}v_{L}, & \text { se } x<0 \\ v_{R}, & \text { se } x \geq 0\end{cases}
$$

em que $v_{L}$ e $v_{R}$ são constantes. Usando a condição de Rankine-Hugoniot para determinar a velocidade de propagação desta descontinuidade, é possível constatar que

$$
S=\frac{G\left(v_{L}\right)-G\left(v_{R}\right)}{v_{L}-v_{R}}=\frac{1}{2} \frac{\left(v_{L}^{2}-v_{R}^{2}\right)}{\left(v_{L}-v_{R}\right)}=\frac{1}{2} \frac{\left(v_{L}-v_{R}\right)\left(v_{L}+v_{R}\right)}{\left(v_{L}-v_{R}\right)}=\frac{v_{L}+v_{R}}{2}
$$

Portanto, a velocidade de propagação da descontinuidade para a equação de Burgers é uma média dos valores à esquerda e à direita da descontinuidade da solução.

\section{Condiçōes de Entropia}

Como vimos anteriormente, soluções fracas não são únicas e portanto resta-nos entender como escolher a solução fraca "correta". Uma maneira de decidir pela solução correta é escolher a solução que é o limite da equação com viscosidade, à qual chamamos de soluçōes com viscosidade nula. A principal razão para a escolha da solução com viscosidade nula é que, na maioria das vezes, as equaçōes que estamos resolvendo se aproximam de uma situação física que inclue algum tipo de dissipação. Portanto, a solução que iremos escolher irá se aproximar de uma solução com pouca dissipação.

À seguir apresentamos duas maneiras distintas de caracterizar a solução entrópica.

(Versão I) A solução da equação (3.16), $v=v(x, t)$, contendo uma descontinuidade propagando-se com velocidade $S$ dada por (3.24) satisfaz a condição de entropia se

$$
G^{\prime}\left(v_{L}\right)>S>G^{\prime}\left(v_{R}\right)
$$


em que $v_{L}$ e $v_{R}$ são valores à esquerda e à direita da descontinuidade da solução, respectivamente. Essa versão é conhecida como condição de entropia de Lax. Ela nos diz que as características partem da condição inicial em direção à descontinuidade e a inclinação dessa descontinuidade situa-se entre as inclinações das características à sua esquerda e à sua direita. Se $G$ é convexa $\left(G^{\prime \prime}>0\right)$ essa condição se reduz a $v_{L}>v_{R}$. No caso em que $G \dot{e}$ côncava $\left(G^{\prime \prime}<0\right)$ a condição de Lax implica em $v_{R}>v_{L}$.

(Versão II) A solução $v(x, t)$ de (3.16) é a solução entrópica se ao longo da descontinuidade vale a relação

$$
\frac{G(v)-G\left(v_{L}\right)}{v-v_{L}} \geq S \geq \frac{G(v)-G\left(v_{R}\right)}{v-v_{R}}
$$

para todo $v$ entre $v_{L}$ e $v_{R}$. A função $G$ não necessariamente é convexa.

Note que se $G$ é convexa, esta condição de entropia reduz-se àquela da Versão I. Existem outras condições de entropia e relações entre elas. Para mais informações veja [44].

Observação 3.2.12. A solução de rarefação dada pelo Exemplo 3.2.7 viola a condição de entropia da Versão I. Para constatar isso, lembramos que $G(v)=\frac{1}{2} v^{2}$ e $G^{\prime}(v)=v$. Pelo Exemplo 3.2.11 sabemos que $S=\frac{v_{L}+v_{R}}{2}$ e portanto para $v_{L}=0, v_{R}=1$ temos $S=\frac{1}{2}$. Assim podemos observar que $G^{\prime}\left(v_{L}\right)>S>G^{\prime}\left(v_{R}\right)$ não se verifica. A Versão II também é violada pois exige que $v \geq 1 \geq v+1$, o que não é verificado.

Como vimos acima, o problema para leis de conservação não é a existência de soluçôes, e sim a unicidade das soluçōes. O teorema que vamos enunciar abaixo, ilustra como a condição de entropia I implica em unicidade.

Teorema 3.2.13. Suponha que $G$ é convexa e que a solução v para o problema de valor inicial

$$
\begin{gathered}
v_{t}+G(v)_{x}=0, \quad x \in \mathbb{R}, \quad t>0 \\
v(x, 0)=v_{0}(x), \quad x \in \mathbb{R}
\end{gathered}
$$

satisfaz a condição de entropia I em toda descontinuidade. Então a solução v é a única solução para o problema de valor inicial acima que satisfaz a condição de entropia I e é uma solução com viscosidade nula (entrópica) para tal problema de valor inicial.

Exemplo 3.2.14. A finalidade deste exemplo é discutir a solução para a lei de conservação (3.25) com condiçâo inicial fornecida pelo Exemplo 3.2.11 onde $v_{L}<v_{R}$ e $G^{\prime}$ é crescente. 
Nos Exemplos 3.2.7 e 3.2.9, mostramos que o problema não tem uma única solução fraca, e que existe uma solução com saltō e uma solução continua contendo um leque de características. A solução descontínua não satisfaz a condição de entropia $I$, enquanto que a soluçâo contínua satisfaz por vacuidade.

Neste exemplo, vamos mostrar que isto é um resultado geral. Temos que

$$
S=\frac{G\left(v_{L}\right)-G\left(v_{R}\right)}{v_{L}-v_{R}}
$$

$e x=\psi(t)$ a solução para

$$
\frac{d \psi}{d t}=S . \quad \operatorname{com} \quad \psi(0)=0
$$

então, analogamente ao Exemplo 3.2.7, a função

$$
v(x, t)= \begin{cases}v_{L}, & \text { se } x \leq S t \\ v_{R}, & \text { se } x>S t\end{cases}
$$

é uma solução fraca para a lei de conservação (3.11) com condiçâo inicial do Exemplo 3.2.11. É fácil ver que esta solução não satisfaz a condiçâo de entropia $I$, pois $v_{L}>S>v_{R} \leftrightarrow v_{L}>$ $v_{R}$ o que contraria a suposição de que $v_{L}<v_{R}$.

As características associadas a este problema são dadas por $x(t)=G^{\prime}\left(v_{0}\right) t+x_{0}$ em que $v_{0}=v\left(x_{0}, 0\right)$ e $x_{0} \in \mathbb{R}$. Existem três situações, excluindo a que já estamos considerando, que podem ocorrer: $G^{\prime}\left(v_{L}\right)$ e $G^{\prime}\left(v_{R}\right)$ serem ambos positivos, $G^{\prime}\left(v_{L}\right)$ e $G^{\prime}\left(v_{R}\right)$ serem ambos negativos, e $G^{\prime}\left(v_{L}\right)<0$ e $G^{\prime}\left(v_{R}\right)>0$. O gráfico das características para esta última situaçâo (veja Figura 3.2.10) apresenta uma região sem quaisquer características devido ao fato de que a inclinação das características à esquerda de $x=0$ ser negativa e da direita de $x=0$ ser positiva.

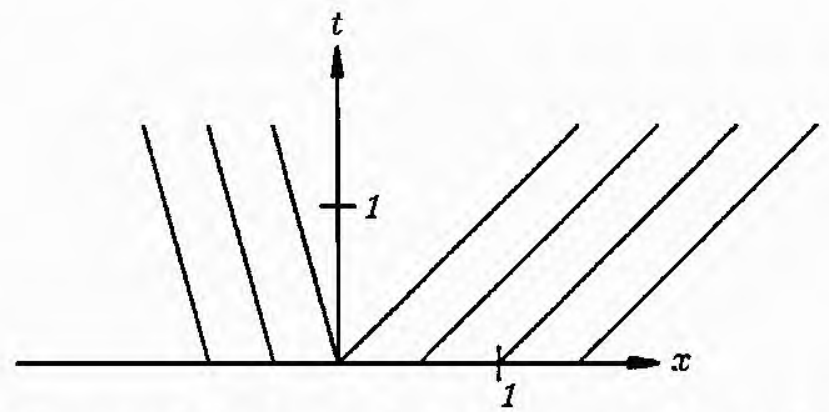

Figura 3.2.10. Características associadas com o problema de valor inicial definido pela lei de consevação (3.11) com condição inicial (3.32) para $v_{L}<v_{R} q u a n d o G^{\prime}\left(v_{L}\right)<0<G^{\prime}\left(v_{R}\right)$.

Nos outros casos, isto acontece devido ao fato de que a inclinaçâo das características ser dada por $1 / G^{\prime}\left(v_{0}\right)$, e como estamos considerando $v_{L}<v_{R}$, temos que $1 / G^{\prime}\left(v_{L}\right)>1 / G^{\prime}\left(v_{R}\right)$. 
O procedimento para encontrar uma solução é preencher a região vazia com um leque de características. Buscamos uma solução da forma

$$
v(x, t)=\psi\left(\frac{x}{t}\right), \quad t>0
$$

a qual é conhecida como solução de similaridade, de maneira que a solução $v$ é constante ao longo de curvas características $x / t=$ cte., no leque. Substituindo (3.35) em (3.11) temos

$$
-\frac{x}{t^{2}} \psi^{\prime}\left(\frac{x}{t}\right)+\frac{1}{t} G^{\prime}\left(\psi\left(\frac{x}{t}\right)\right) \psi^{\prime}\left(\frac{x}{t}\right)=0
$$

impondo que $\psi^{\prime}\left(\frac{x}{t}\right) \neq 0$, para todo $(x, t)$, obtemos

$$
G^{\prime}\left(\psi\left(\frac{x}{t}\right)\right)=\frac{x}{t}
$$

Assim, para uma solução da forma (3.35) satisfazer (3.11), $\psi$ deve ser a inversa da função $G^{\prime}$, isto é, $\psi\left(\frac{x}{t}\right)=G^{-1}\left(\frac{x}{t}\right)$.

Notamos que quando $x=G^{\prime}\left(v_{L}\right) t$. (a curva caracteristica do lado esquerdo do leque) fornece

$$
\psi\left(\frac{x}{t}\right)=\psi\left(G^{\prime}\left(v_{L}\right)\right)=G^{-1}\left(G^{\prime}\left(v_{L}\right)\right)=v_{L}
$$

e quando $x=G^{\prime}\left(v_{R}\right) t$ (a curva característica do lado direito do leque) fornece

$$
\psi\left(\frac{x}{t}\right)=\psi\left(G^{\prime}\left(v_{R}\right)\right)=G^{\prime-1}\left(G^{\prime}\left(v_{R}\right)\right)=v_{R}
$$

Portanto, se definirmos uma soluçâo como sendo

$$
v(x, t)= \begin{cases}v_{L} & \text { se } x<G^{\prime}\left(v_{L}\right) t \\ G^{\prime-1}\left(\frac{x}{t}\right) & \text { se } G^{\prime}\left(v_{L}\right) t \leq x \leq G^{\prime}\left(v_{R}\right) t \\ v_{R} & \text { se } x>G^{\prime}\left(v_{R}\right) t,\end{cases}
$$

temos uma solução fraca para o problema (3.11) com condição inicial dada no Exemplo 3.2.11. A solução (3.36) satisfaz as condições de entropia I e II trivialmente porque não tem descontinuidades.

Como vimos, os fenòmenos regidos por equações hiperbólicas podem apresentar dificuldades as mais diversas. Algumas propriedades especiais que terão ainda de ser levadas em conta na solução numérica são: As informações se propagam ao longo das características com velocidade finita; os fenômenos regidos por esse tipo de equação não possuem mecanismos dissipativos; mesmo se os dados iniciais forem suaves, a solução numérica admite descontinuidades. 
Todas as propriedades acima devem ser consideradas pelos métodos numéricos para fornecer respostas representativas do problema físico em questão. Estes devem analisar direções e velocidades de propagação de informações, ao longo do domínio computacional, e não introduzir efeitos dissipativos. Essas observaçōes serão os tópicos abordados nas próximas seções.

\subsection{Esquemas Numéricos para Leis de Conservação}

Nesta seção apresentamos as discretizações por diferenças finitas para a equação da onda linear e não linear. Em seguida, fornecemos discretizações e esquemas numéricos para uma equação diferencial hiperbólica na forma conservativa. Estaremos discutindo métodos para uma lei de conservação escalar.

Aproximações por diferenças finitas podem ser obtidas de várias formas. As mais comuns são expansão em série de Taylor e interpolaçâo polinomial. Nos concentraremos nas aproximações por expansão em série de Taylor para a obtenção de fórmulas para as primeiras e segundas derivadas de uma função $v(x, t)$ nas variáveis espacial e temporal.

Como estamos interessados em obter uma expressão que relacione a variação de $v(x, t)$ com incrementos em $x$ e $t$, separadamente em cada variável, vamos usar a expansão em série de Taylor de funçôes de duas variáveis

$$
\begin{aligned}
v(x \pm \Delta x, t) & =v(x, t)+( \pm \Delta x) v_{x}(x, t)+\frac{( \pm \Delta x)^{2}}{2} v_{x x}(x, t)+o(\Delta x)^{3} . \\
v(x, t \pm \Delta t) & =v(x, t)+( \pm \Delta t) v_{t}(x, t)+\frac{( \pm \Delta t)^{2}}{2} v_{t t}(x, t)+o(\Delta t)^{3} .
\end{aligned}
$$

Isolando a primeira derivada tanto na variável espacial como na temporal, temos

$$
\begin{aligned}
& v_{x}(x, t)=\frac{ \pm v(x \pm \Delta x, t) \mp v(x, t)}{\Delta x}+o(\Delta x) . \\
& v_{t}(x, t)=\frac{ \pm v(x, t \pm \Delta t) \mp v(x, t)}{\Delta t}+o(\Delta t) .
\end{aligned}
$$

Escrevendo (3.37) para ambos os sinais e subtraindo uma dessas fórmulas da outra, obtemos uma fórmula para a derivada espacial, a saber

$$
v_{x}(x, t)=\frac{v(x+\Delta x, t)-v(x-\Delta x, t)}{2 \Delta x}+o(\Delta x)^{2} .
$$


O mesmo pode ser feito para a derivada temporal, fornecendo

$$
v_{t}(x, t)=\frac{v(x, t+\Delta t)-v(x, t-\Delta t)}{2 \Delta t}+o(\Delta t)^{2} .
$$

Agora, como fizemos antes, isolamos a segunda derivada na variável espacial e temporal na série de Taylor e procedemos analogamente como fizemos para (3.41) e (3.42), para obter

$$
\begin{aligned}
& v_{x x}(x, t)=\frac{v(x+\Delta x, t)-2 v(x, t)+v(x-\Delta x, t)}{(\Delta x)^{2}}+o(\Delta x)^{2} . \\
& v_{t t}(x, t)=\frac{v(x, t+\Delta t)-2 v(x, t)+v(x, t-\Delta t)}{(\Delta t)^{2}}+o(\Delta t)^{2} .
\end{aligned}
$$

As aproximaçōes (3.39) e (3.40) são conhecidas como diferenças progressivas e regressivas para as variáveis espacial e temporal. As equações (3.41) e (3.42) são conhecidas como diferenças centrais no espaço e no tempo para a primeira derivada. Finalizando, (3.43) e (3.44) são também diferenças centrais mas para a segunda derivada. Definimos os operadores para cada uma das aproximações na variável espacial, por:

$$
\begin{aligned}
D^{+x} v & \equiv \frac{v(x+\Delta x, t)-v(x, t)}{\Delta x}, \\
D^{-x} v & \equiv \frac{v(x, t)-v(x-h, t)}{\Delta x}, \\
D^{0 x} v & \equiv \frac{v(x+h, t)-v(x-h, t)}{2 \Delta x}, \\
D^{+x-x} v & \equiv \frac{v(x+h, t)-2 v(x, t)+v(x-h, t)}{\Delta x^{2}} .
\end{aligned}
$$

Analogamente para a variável temporal.

A solução numérica de uma EDP é feita sobre um conjunto de pontos discretos do domínio de cálculo. A esse conjunto de pontos discretos dá-se o nome de malha. Para aproximar a equação $v_{t}+a v_{x}=0$ seguindo o enfoque acima, discretizamos uma regiāo do plano- $(x, t)$ em uma coleção de pontos separados entre si por uma distância $\Delta x=h$ e $\Delta t=k$, não necessariamente iguais (veja Figura 3.3.2). Assim todo ponto da malha pode ser representado pelo par de coordenadas $(i, n)$ correspondendo ao ponto $\left(x_{0}+i \Delta x, y_{0}+n \Delta t\right)$, onde o ponto $\left(x_{0}, y_{0}\right)$ representará a origem do sistema de coordenadas, tomado aqui por $(0,0)$. Seja $u_{i}^{n}$ uma aproximação de $v(i \Delta x, n \Delta t)$. Então temos os seguintes esquemas que aproximam a equação da onda linear.

(i) Esquema progressivo: $u_{i}^{n+1}=u_{i}^{n}-a k D^{+x} u_{i}^{n}$; se ambas as derivadas temporal e espacial forem aproximadas por diferenças progressivas. 
(ii) Esquema regressivo: $u_{i}^{n+1}=u_{i}^{n}-a k D^{-x} u_{i}^{n}$; se a derivada temporal for aproximada por diferenças progressivas e a derivada espacial por diferenças regressivas.

(iii) Esquema centrado: $u_{i}^{n+1}=u_{i}^{n}-a k D^{0 x} u_{i}^{n}$; se a derivada temporal for aproximada por diferenças progressivas e a derivada espacial por diferenças centrais.

Uma pergunta natural seria: qual dessas aproximações produz melhor resultado? A resposta é obtida através da análise dos domínios de dependência e influência e das características da EDP. Lembremos que quando $a>0$ a informação propaga-se da esquerda para a direita (veja Figura 3.2.1).

Vamos em seguida considerar algumas propriedades dos operadores que aproximam a primeira derivada com o objetivo de responder a pergunta acima.

- $D^{+x}$ : calcula uma aproximação para a derivada em $i$, usando informações em $i$ e $i+1$; portanto a informação para a solução propaga da direita para a esquerda.

- $D^{-x}$ : calcula uma aproximação para a derivada em $i$, usando informaçōes em $i$ e $i-1$; portanto a informação para a solução propaga da esquerda para a direita.

- $D^{0 x}$ : calcula uma aproximação para a derivada em $i$, usando informações em $i+1$ e $i-1$; portanto a informação para a solução propaga para ambos os lados.

Uma reflexão cuidadosa sobre o observado acima leva-nos a concluir que o esquema de diferenças progressivas não é adequado pois sabemos que no caso contínuo a informação é transportada da esquerda para a direita e espera-se que o método numérico faça o mesmo. Assim diferenças progressivas não são apropriadas quando $a>0$. O método de diferenças regressivas para esse exemplo é chamado de esquema upwind, pois us a valores a montante (direção de onde correm as águas duma corrente fluvial) na direção de propagação, o que é desejável. Métodos upwind serão mencionados na próxima seção. O método em que o termo convectivo $\left(a v_{x}\right)$ é discretizado por diferenças centrais, introduz uma depêndencia artificial entre o ponto $\left(x_{i}, t_{n+1}\right)$ e os pontos $\left(x_{i+1}, t_{n}\right)$ e $\left(x_{i}, t_{n}\right)$. Dessa maneira, o valor em $u_{i+1}^{n}$ passa a depender dos valores de $u_{i+1}^{n}$ e $u_{i}^{n}$ fora da característica o que não é adequado, uma vez que as informaçōes se propagam ao longo das características. Além disso, a aproximação por diferenças centrais por depender de 3 pontos no nível $n$, permite somente o cálculo de valores interiores no nível de tempo $n+1$, sendo necessária a especificação de 2 condições de fronteira, uma à direita e outra à esquerda. No caso da equaçāo de advecção, a prescrição de 2 condições de fronteira não é fisicamente correta uma vez que as características "levam"informações da fronteira para o interior do domínio. Vide Figura 3.3.1. 


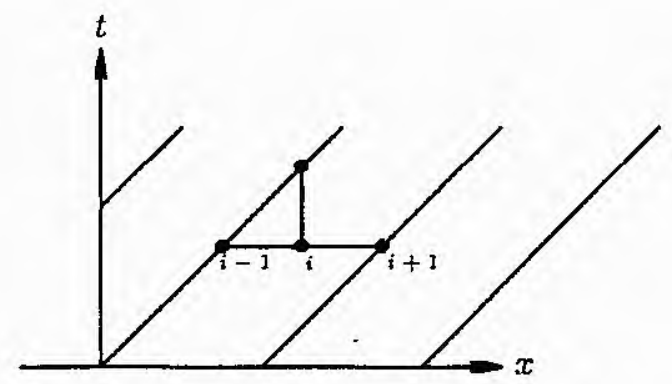

Figura 3.3.1. Estêncil da discretização por diferenças centrais sobreposto às curvas características da Equação (3.1) com $a>0$.

Realmente, embora esse método tenha erro de truncamento de ordem maior, isto é, $o\left(\Delta t+\Delta x^{2}\right)$, ele é um típico exemplo no qual, discretizaçôes aparentemente "mais precisas", não têm o mesmo comportamento na prática.

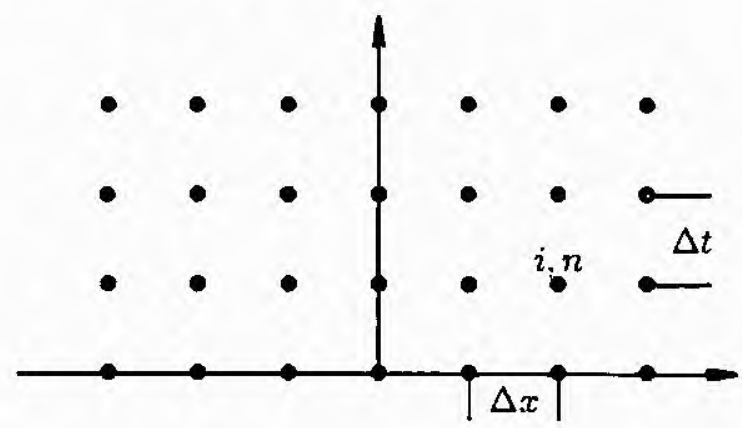

Figura 3.3.2. . Malha computacional.

Um outro fato que não devemos desconsiderar deriva, como já dissemos, do conceito de domínios de dependência e influência. Uma das propriedades relevantes de equaçōes hiperbólicas é que devido à velocidade finita de propagação das informações através do domínio computacional, a solução possui um domínio finito de dependência. A definição abaixo faz uma comparação entre o domínio analítico de dependência e o domínio numérico de dependência.

Definição 3.3.1. Um esquema de diferenças derivado de uma equação diferencial parcial é dito satisfazer a condição de Courant-Friedrichs-Lewy (CFL) se o domínio analítico de dependência, $D_{a}$, está contido no domínio numérico de dependência, $D_{n}$. desse método.

Como exemplo. considere o problema de valor inicial (3.1)-(3.2). Para $a>0$ sabemos que a solução no ponto $(x, t)$ depende unicamente de valores de $f$ no ponto $x_{0}$ onde $x_{0}=x-a t$. $\mathrm{O}$ ponto $x_{0}$ é chamado de domínio de dependência do ponto $(x, t)$. Agora, iremos definir o 
domínio de dependência numérico de um ponto para um esquema de diferenças que é solução do problema (3.1)-(3.2). Considere o esquema

$$
u_{i}^{n+1}=u_{i}^{n}-R\left(u_{i}^{n}-u_{i-1}^{n}\right)
$$

em que $R=a \Delta t / \Delta x$. A solução numérica em um ponto $(i \Delta x,(n+1) \Delta t)$ utilizando esse esquema dependerá dos pontos $((i-n-1) \Delta x, 0), \ldots,(i \Delta x, 0)$ situados no nível de tempo zero. É digno de nota (veja Figura 3.3.3) que mesmo se valores distintos para $\Delta x$ e $\Delta t$ forem usados, digamos $\delta x$ e $\delta t$, contanto que $a \delta t / \delta x=R$ e $\left(i_{1} \delta x,\left(n_{1}+1\right) \delta t\right)=(i \Delta x,(n+1) \Delta t)$ é um ponto da malha, a solução em $(i \Delta x,(n+1) \Delta t)$ ainda dependerá dos pontos começando em $((i-n-1) \Delta x, 0)$ e finalizando em $(i \Delta x, 0)$. Por esta razão, definimos o domínio numérico de dependência do ponto $(i \Delta x,(n+1) \Delta t)$ em relação ao esquema regressivo (3.49) como sendo o intervalo $\left.D_{n}=[(i-n-1) \Delta x, i \Delta x)\right]$.

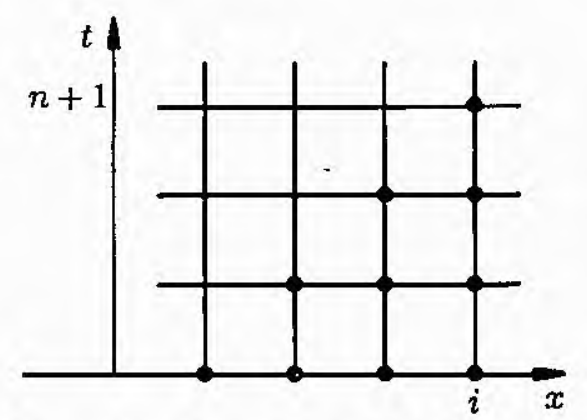

Figura 3.3.3. Domínio numérico de dependência do ponto $\left(i_{1} \delta x,\left(n_{1}+1\right) \delta t\right)=(i \Delta x,(n+$ 1) $\Delta t$ ) em relação ao esquema de diferenças (3.49) onde $R=a \Delta t / \Delta x$.

O domínio analítico de dependência do ponto $(x, t)$ para a equação diferencial parcial (3.1) é $x_{0}=x-a t$. Se considerarmos o ponto $(x, t)=(i \Delta x,(n+1) \Delta t)$, então $x_{0}=x-a t=i \Delta x-$ $a(n+1) \Delta t=[i-R(n+1)] \Delta x$. Assim vemos que se $x_{0} \in[(i-n-1) \Delta x, i \Delta x]$ (que é o domínio numérico de dependência do esquema (3.49)) então $(i-n-1) \Delta x \leq[i-R(n+1)] \Delta x \leq i \Delta x$, e portanto

$$
i-n-1 \leq i-R(n+1) \leq i \leftrightarrow-(n+1) \leq-R(n+1) \leq 0 \leftrightarrow 1 \geq R \geq 0
$$

mostrando que o domínio analítico de dependência está contido no domínio numérico de dependência se e somente se $0 \leq R \leq 1$. Assim concluímos que a condição CFL para a equação diferencial parcial (3.1) e para o esquema (3.49) é equivalente à desigualdade $0 \leq R \leq 1$.

Métodos explícitos devem satisfazer a condição CFL $\leq 1$, o que efetivamente proíbe a propagação de informações por uma distância maior que $\Delta x$ a cada instante de tempo. Isso 
pode ser deduzido se observarmos que

$$
\mathrm{CFL}=|a| \frac{\Delta t}{\Delta x} \leq 1 \Rightarrow|a|(\Delta t) \leq \Delta x .
$$

\subsubsection{Esquemas Upwind}

Vamos continuar examinando a equação da onda linear e não linear. Considere novamente a equação da onda da forma

$$
v_{t}+a(x) v_{x}=0
$$

onde a velocidade de propagação é uma função $a(x)$ da posição. Podemos nos basear no que foi feito para o caso linear e construir variações do esquema upwind anterior. A direção de propagação dependerá do sinal de $a$. Por exemplo, o esquema

$$
u_{i}^{n+1}=u_{i}^{n}-\Delta t,\left[\max \left(0, a_{i}\right) D^{-x} u_{i}^{n}+\min \left(0, a_{i}\right) D^{+x} u_{i}^{n}\right]
$$

escolhe a direção correta de upwinding de tal maneira que o esquema de diferenças sempre inclue o domínio analítico de dependência no seu domínio numérico. Esse esquema funciona da seguinte maneira: quando a velocidade de propagação $a$ é positiva (como no caso constante), a informação viaja da esquerda para a direita, e assim o esquema regressivo é utilizado; quando a é negativo, o esquema progressi vo é escolhido. Analogamente ao caso da velocidade de propagação constante, a condição CFL é dada por $\frac{k}{h} \leq \frac{1}{|a|}$, quando a velocidade de propagação é variável.

O esquema (3.52) para $a(x)>0$ ou $a(x)<0, \forall x$ tem uma extensão natural para o caso não linear quando a função de fluxo $G$ é monótona, a saber,

$$
u_{i}^{n+1}= \begin{cases}u_{i}^{n}-R\left\{G\left(u_{i}^{n}\right)-G\left(u_{i-1}^{n}\right)\right\}, & \text { se } G^{\prime}\left(u_{i}^{n}\right)>0 \\ u_{i}^{n}-R\left\{G\left(u_{i+1}^{n}\right)-G\left(u_{i}^{n}\right)\right\}, & \text { se } G^{\prime}\left(u_{i}^{n}\right) \leq 0 .\end{cases}
$$

ou

$$
u_{i}^{n+1}= \begin{cases}u_{i}^{n}-R\left\{G\left(u_{i}^{n}\right)-G\left(u_{i-1}^{n}\right)\right\}, & \text { se } a_{i+1 / 2}^{n}>0 \\ u_{i}^{n}-R\left\{G\left(u_{i+1}^{n}\right)-G\left(u_{i}^{n}\right)\right\}, & \text { se } a_{i+1 / 2}^{n} \leq 0\end{cases}
$$

onde

$$
a_{i+1 / 2}^{n}= \begin{cases}\frac{G\left(u_{i+1}^{n}\right)-G\left(u_{i}^{n}\right)}{u_{i+1}^{n}-u_{i}^{n}}, & \text { se } u_{i+1}^{n}-u_{i}^{n} \neq 0 \\ G^{\prime}\left(u_{i}^{n}\right), & \text { se } u_{i+1}^{n}-u_{i}^{n}=0 .\end{cases}
$$


Quando $G^{\prime}>0\left(a_{i+1 / 2}^{n}>0\right)$ o esquema (3.53) recebe o nome de esquema FTBS, ou seja, progressivo no tempo e regressivo no espaço. Caso contrário, é chamado FTFS (progressivo no tempo e progressivo no espaçö). A diferença entre os esquemas (3.53) c (3.54) é quc o esquema (3.54) usa uma aproximação discreta da derivada $G^{\prime}(u)$ dada por (3.55). A referência [43] mostra através de exemplos numéricós que o esquema (3.53) não é conservativo (veja Definição 3.3.3) e que o esquema (3.54) não aproxima a solução entrópica. Realmente, esses são aspectos negativos desses esquemas, mas eles serão úteis no desenvolvimento de esquemas de alta resolução. No casso geral com $G$ não monótona, os esquemas de Godunov e de Engquist-Osher que veremos a seguir fornecerão extensões apropriadas de esquema upwind.

Na derivação de esquemas numéricos para leis de conservação, a condição $C F L$ também será relevante para a análise de tais esquemas. Isso é o que faremos a seguir.

Considere a lei de conservação e condição inicial

$$
\begin{aligned}
v_{t}+G(v)_{x} & =0, x \in \mathbb{R}, \quad t>0 \\
v(x, 0) & =f(x), x \in \mathbb{R}
\end{aligned}
$$

e o esquema de diferenças de três pontos

$$
u_{i}^{n+1}=W\left(u_{i-1}^{n}, u_{i}^{n}, u_{i+1}^{n}\right)
$$

Seja $P=(i \Delta x,(n+1) \Delta t)$ um ponto da malha. Como mostramos para o caso linear, o domínio numérico de dependência do esquema de diferenças (3.58) é o intervalo [ $i-n-$ 1) $\Delta x,(i+n+1) \Delta x]$. Se a solução $v$ para o problema de valor inicial (3.56)-(3.57) é suave, $v$ pode ser escrita como $v(x, t)=f\left(x-G^{\prime}(v(x, t)) t\right)$. Como no caso linear dizemos que a condição CFL é verificada se $D_{a} \subset D_{n}$, isto é se

$$
(i-n-1) \Delta x \leq i \Delta x-G^{\prime}(v(i \Delta x,(n+1) \Delta t))(n+1) \Delta t \leq(i+n+1) \Delta x
$$

ou

$$
-1 \leq-R G^{\prime}(v(i \Delta x,(n+1) \Delta t)) \leq 1
$$

ou

$$
R\left|G^{\prime}(v(i \Delta x,(n+1) \Delta t))\right| \leq 1
$$


Assim, impondo que $R$ satisfaça

$$
R \max \left|G^{\prime}\right| \leq 1
$$

a condiçâo (3.59) é verificada para todo $i$ e $n$. Extensões para várias variáveis segue de maneira análoga. Geralmente podemos precisar da versão discreta da condição CFL em vez de (3.60). A condição CFL não linear, discreta é dada por

$$
R \max _{i, n}\left|a_{i+1 / 2}^{n}\right| \leq 1
$$

em que $a_{i+1 / 2}^{n}$ é dado em (3.55).

A seguir, vamos apresentar extensões dos esquemas upwind para o caso em que $G$ não é monótona e na sequência deduzimos dois esquemas de segunda ordem para a lei de conservação (3.11).

\subsubsection{Esquemas de Gódunov e de Engquist-Osher}

É desejável que os esquemas numéricos produzam soluções que satisfaçam as condições de entropia. Os métodos de viscosidade artificial escolhem a solução entrópica desde que adicionemos um termo de suavização conforme mostrado em (3.10). No entanto, a suavização destrói estruturas como cantos e singularidades. O objetivo é encontrar métodos que evitam a existência de soluções violando a condição de entropia e que apresentem pouca difusão.

O Método de Godunov [16] satisfaz os requisitos acima. Este método é baseado na solução exata de problemas de Riemann locais e os utiliza para obter um método de diferenças finitas upwind que preserva monotonicidade. Como veremos mais a frente, uma dificuldade associada com a aproximação numérica de problemas com soluções descontínuas é que oscilações podem aparecer próximas as descontinuidades. Os esquemas monotônicos tendem a amortecer essas oscilações, daí a sua importância prática. Antes de derivarmos o método de Godunov para a equação conservativa (3.11) com $G$ uma função escalar convexa, forneceremos três importantes definições:

Definição 3.3.2. Um problema de Riemann é constituído de uma equação diferencial parcial na forma conservativa tendo como condição inicial a função de Heaviside, ou seja:

$$
\begin{aligned}
v_{t}+[G(v)]_{x} & =0 \\
v(x, 0) & =\operatorname{Heav}(x)= \begin{cases}v_{L}, & \text { se } x<0 \\
v_{R}, & \text { se } x \geq 0\end{cases}
\end{aligned}
$$


Definição 3.3.3. Um esquema de discretização explícito de (3.11) dado por

$$
u_{i}^{n+1}=W\left(u_{i-p-1}^{n}, \ldots, u_{i+q}^{n}\right), \quad \forall n \geq 0, i \in \mathbb{Z},
$$

em que $W: \mathbb{R}^{q+p+2} \rightarrow \mathbb{R}$ é uma função contínua, pode ser posto na forma conservativa se existe uma função de fluxo numérico $g: \mathbb{R}^{q+p+1} \rightarrow \mathbb{R}$ tal que

$$
u_{i}^{n+1}=u_{i}^{n}-R\left[g_{i+1 / 2}^{n}-g_{i-1 / 2}^{n}\right],
$$

em que $g_{i+1 / 2}^{n}=g\left(u_{i-p}^{n}, \ldots, u_{i+q}^{n}\right)$ e $R=\frac{\Delta t}{\Delta x}$.

A definição acima implica que um esquema conservativo satisfaz:

$$
-\sum_{i=-\infty}^{+\infty} u_{i}^{n+1}=\sum_{i=-\infty}^{+\infty} u_{i}^{n}
$$

ou seja, o total da quantidade $u$ não varia dō tempo $t_{n}$ para $t_{n+1}$. De fato, todo esquema numérico na forma (3.65) é conservativo, pois

$$
\begin{aligned}
\sum_{i=-\infty}^{+\infty} u_{i}^{n+1} & =\sum_{i=-\infty}^{+\infty} u_{i}^{n}-R\left[\sum_{i=-\infty}^{+\infty}\left(g_{i+1 / 2}-g_{i-1 / 2}\right)\right] \\
& =\sum_{i=-\infty}^{+\infty} u_{i}^{n}-R\left(\cdots+g_{-3 / 2}+g_{1 / 2}+\ldots-g_{-3 / 2}-g_{1 / 2}-\ldots\right)=\sum_{i=-\infty}^{+\infty} u_{i}^{n} .
\end{aligned}
$$

A primeira propriedade básica que um esquema como (3.65) deve satisfazer é a de consistência com a lei de conservação (3.11). Observe que

$$
\left(u_{i}^{n+1}-u_{i}^{n}\right) / \Delta t
$$

é uma aproximação de $u_{t}$ no ponto $\left(x_{i}, t_{n}\right)$ da malha. Assim impomos que

$$
\left(g\left(u_{i-p}^{n}, \ldots, u_{i+q}^{n}\right)-g\left(u_{i-p-1}^{n}, \ldots, u_{i+q-1}^{n}\right)\right) / \Delta x
$$

seja uma aproximação de $G(v)_{x}$ no mesmo ponto da malha. Isto leva à seguinte definição.

Definição 3.3.4. O esquema de diferenças (3.64) é consistente com a equação (3.11) se

$$
g(v, \ldots, v)=G(v), \quad \forall v \in \mathbb{R},
$$

a menos de uma constante aditiva. 
Observação 3.3.5. Para consistência, é suficiente ter g uma função Lipschitz continua em cada variável. Dizemos que g é Lipschitz em $v$ se existe uma constante $K \geq 0$ (que pode depender de v) tal que

$$
|g(u, w)-G(v)| \leq K \max \{|u-v|,|w-v|\}
$$

para todo $u, w$ com $|u-v|,|w-v|$ suficientemente pequeno. Dizemos que $g$ é uma função Lipschitz contínua se ela é Lipschitz em todo ponto.

Para começarmos a descrever o método de Godunov, dividiremos o eixo $x$ em intervalos de comprimento $h$, em que o i-ésimo intervalo $I_{i}=\left[x_{i}, x_{i+1}\right]$ terá centro no ponto $x_{i+1 / 2}=$ $x_{i}+\frac{h}{2}$. Vamos representar por $\bar{u}^{n}$ a solução aproximada, constante por partes; para a lei de conservação

$$
v_{t}+[G(v)]_{x}=0
$$

com condição inicial $v(x, 0)=f(x), x \in \mathbb{R}$ no tempo $t=n \Delta t$. A função $\ddot{u}^{n}$ será constante sobre o intervalo $I_{i}^{1 / 2}=\left[x_{i-1 / 2}, x_{i+1 / 2}\right]$ e iremos denotar o valor de $\bar{u}^{n}$ em $x=i \Delta x$ por $u_{i}^{n}$. Denotaremos a solução para a lei de conservação $(3.67)$ com condição inicial $v\left(x, t_{n}\right)=$ $\bar{u}^{n}(x), x \in \mathbb{R}$, por $\bar{U}=\bar{U}(x, t)$. Estamos interessados em usar $\bar{U}(x, t)$ em $t_{n+1}$ para definir a aproximação para a solução no tempo $t=t_{n+1}, \bar{u}^{n+1}$, como a média

$$
u_{i}^{n+1}=\frac{1}{\Delta x} \int_{x_{i-1 / 2}}^{x_{i+1 / 2}} \bar{U}\left(x, t_{n+1}\right) d x,
$$

ou seja, a solução constante por partes no tempo $t_{n+1}$, será a média da solução do problema de Riemann tendo como condição inicial a aproximação no tempo $t_{n}$.

Quando aproximamos a condição inicial por funções constantes em cada intervalo $I_{i}$, essa condição, outrora, possivelmente contínua, passa a apresentar descontinuidades (saltos). É fácil imaginar que alguns desses saltos irão se tornar leques $\left(u_{L}<u_{R}\right)$ e outros choques $\left(u_{L}>u_{R}\right)$. Para $\Delta t$ suficientemente pequeno a solução vai, localmente, constituir-se de uma série de choques e leques conectados por partes de funções constantes. Alguns dos choques e leques moverão e outros ficarão estácionarios. Os que se movimentarão, geralmente, moverāo com velocidades e direções diferentes dependendo da função de fluxo $G$. Se a condição CFL, $\Delta t / \Delta x \max \left|G^{\prime}\left(u_{i}^{n}\right)\right|<1 / 2$ for satisfeita para todo $i$, então os vários choques e leques não iteragirão com as características de problemas vizinhos entre os tempos $t_{n}$ e $t_{n+1}$ (veja Figura 3.3.4). Se considerarmos $\Delta t / \Delta x \max \left|G^{\prime}\left(u_{i}^{n}\right)\right|<1$ para todo $i$, essas características 
interagirão com características de problemas de Riemann vizinhos, mas, a interação vai estar contida em $I_{i}$.

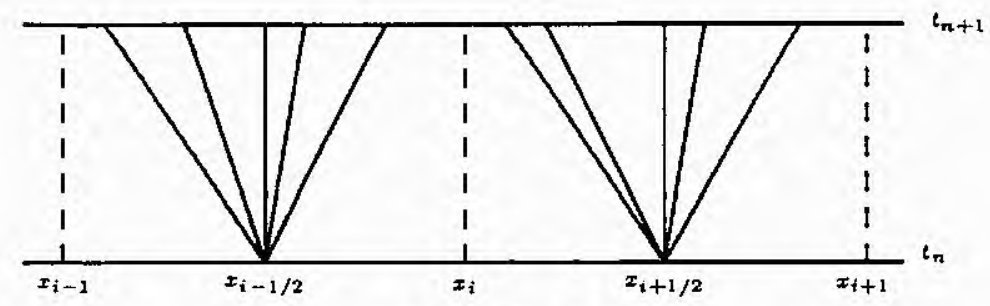

Figura 3.3.4.

Continuamos o processo para obter $u_{i}^{n+1}$ resolvendo os problemas de Riemarin associados com cada lado dos intervalos, $x_{i-1 / 2}, i=-\infty, \ldots, \infty$. Temos os problemas de Riemann

$$
\begin{gathered}
v_{t}+[G(v)]_{x}=0, \quad x \in \mathbb{R}, \quad t>t_{n} \\
v\left(x, t_{n}\right)= \begin{cases}u_{i-1}^{n}, & \text { se } x<x_{i-1 / 2} \\
u_{i,}^{n}, & \text { se } x \geq x_{i-1 / 2} .\end{cases}
\end{gathered}
$$

Integrando a lei de conservação (3.69) com respeito a $x$ e $t$ de $x_{i-1 / 2}$ a $x_{i+1 / 2}$ e $t_{n}$ a $t_{n+1}$, respectivamente e resolvendo a integral primeiro em relação a $t$ e depois em relação a $x$, obtemos

$$
\int_{x_{i-1 / 2}}^{x_{i+1 / 2}}\left[\bar{U}\left(x, t_{n+1}\right)-\bar{U}\left(x, t_{n}\right)\right] d x+\int_{t_{n}}^{t_{n+1}}\left[G\left(\bar{U}\left(x_{i+1 / 2}, t\right)\right)-G\left(\bar{U}\left(x_{i-1 / 2}, t\right)\right)\right] d t=0
$$

onde substituimos $v$ em (3.69) por $\bar{U}$, pois estamos usando $\bar{U}=\vec{U}(x, t)$ para denotar a solução do problema de valor inicial (3.67) com condição inicial $\bar{U}\left(x, t_{n}\right)=\bar{u}^{n}(x)$, isto é, a solução local para o problema de Riemann (3.69)-(3.70). Multiplicando (3.71) por 1/ $\Delta x$ e usando (3.68), podemos escrever essa equação como

$$
u_{i}^{n+1}=u_{i}^{n}-\frac{1}{\Delta x} \int_{t_{n}}^{t_{n+1}}\left[G\left(\bar{U}\left(x_{i+1 / 2}, t\right)\right)-G\left(\bar{U}\left(x_{i-1 / 2}, t\right)\right)\right] d t .
$$

Como sabemos que a solução em $x=x_{i+1 / 2}$ dependerá de $u_{i}^{n}$ e $u_{i+1}^{n}$ (em $x=x_{i-1 / 2}$ dependerá de $u_{i-1}^{n}$ e $u_{i}^{n}$ ), definimos a função de fluxo numérico como sendo

$$
g_{i \pm 1 / 2}^{n}=\frac{1}{\Delta t} \int_{t_{n}}^{t_{n+1}} G\left(\bar{U}\left(x_{i \pm 1 / 2}, t\right)\right) d t
$$

que se substituído em (3.72) fornece o esquema conservativo de Godunov

$$
u_{i}^{n+1}=u_{i}^{n}-R\left[g_{i+1 / 2}^{n}-g_{i-1 / 2}^{n}\right]
$$


em que $R=\Delta t / \Delta x$.

A solução $\bar{U}$ é uma solução de similaridade para o problema de Riemann (3.69)-(3.70), isto é, $\bar{U}$ pode ser escrita como $\bar{U}(x, t)=\psi\left(\left(x-x_{i-1 / 2}\right) /\left(t-t_{n}\right)\right)$. Portanto, em $x=x_{i-1 / 2}$, $\breve{U}\left(x_{i-1 / 2}, t\right)=\psi(0)$ é constante em $t \in\left[t_{n}, t_{n+1}\right]$. Usando este fato, a integral na definição de $g_{i-1 / 2}^{n}$ em (3.73) pode ser simplificada, e fornece

$$
g_{i-1 / 2}^{n}=G\left(\bar{U}\left(x_{i-1 / 2}, t\right)\right)
$$

Uma expressão análoga pode ser obtida para $g_{i+1 / 2}^{n}$.

A dificuldade mais relevante da aplicação do método de Godunov se baseia no fato de que esse método exige a solução exata de uma sequência de problemas de Riemann a cada passo no tempo, o que na prática acarreta um alto custo numérico. Uma maneira de superar essa dificuldade consiste em recorrer aos métodos Riemann solvers os quais não tratamos nessa dissertação (veja $[15,26,28,34,44]$ ). Uma propriedade favorável desse método é que ele produz uma solução que satisfaz a condição de entropia com relativamente pouca difusividade. Estaremos mais à frente mencionando outras propriedades desse método.

Exemplo 3.3.6. O método de Godunov torna-se muito simples para o caso de Burgers, pois nesse caso temos as soluções exatas dos diversos problemas de Riemann. Assim, para determinarmos a função de fluxo numérico para o esquema de Godunov para o problema de Burgers, $G(v)=v^{2} / 2$, nos basearemos nos Exemplos 3.2.4, 3.2.9, 3.2.11 e 3.2.14 constatando que:

se $u_{i \rightarrow 1}^{n} \geq u_{i}^{n}$, a solução para o problema de Riemann (3.69)-(3.70) é

$$
v(x, t)= \begin{cases}u_{i-1}^{n}, & s e \frac{x-x_{i-1 / 2}}{t-t_{n}}<S \\ u_{i}^{n}, & s e \frac{x-x_{i-1 / 2}}{t-t_{n}} \geq S .\end{cases}
$$

se $u_{i-1}^{n}<u_{i}^{n}$, a solução para o problema de Riemann (3.6g)-(3.70) é

$$
v(x, t)= \begin{cases}u_{i-1}^{n}, & \text { se } \frac{x-x_{i-1 / 2}}{t-t_{n}}<u_{i-1}^{n} \\ \frac{x-x_{i-1 / 2}}{t-t_{n}}, & \text { se } u_{i-1}^{n} \leq \frac{x-x_{i-1 / 2}}{t-t_{n}} \leq u_{i}^{n} \\ u_{i}^{n}, & \text { se } \frac{x-x_{i-1 / 2}}{t-t_{n}}>u_{i}^{n} .\end{cases}
$$

Considerando a solução (3.76), se $u_{i-1}^{n} \geq u_{i}^{n}$ então $\bar{U}\left(x_{i-1 / 2}, t\right)=u_{i-1}^{n}$ se $S>0$ e $\vec{U}\left(x_{i-i / 2}, t\right)=u_{i}^{n}$ se $S \leq 0$. Usando a solução (3.77), se $u_{i-1}^{n}<u_{i}^{n}$, então $\bar{U}\left(x_{i-i / 2}, t\right)=u_{i-1}^{n}$ 
se $u_{i-1}^{n}>0, \bar{U}\left(x_{i-i / 2}, t\right)=0$ se $u_{i-1}^{n} \leq 0 \leq u_{i}^{n}$, e $\bar{U}\left(x_{i-i / 2}, t\right)=u_{i}^{n}$ se $u_{i}^{n}<0$. Utilizando (3.75), podemos determinar a função de fluxo numérico associada com o esquema de Godunov para a equação de Burgers, dada como se segue.

Se $u_{i-1}^{n}<u_{i}^{n}$, então

(i) completamente supersônico/subsônico

se $u_{i-1}^{n}>0$, temos $g_{i-1 / 2}^{n}=\frac{1}{2}\left(u_{i-1}^{n}\right)^{2}$

se $u_{i}^{n}<0$, temos $g_{i-1 / 2}^{n}=\frac{1}{2}\left(u_{i}^{n}\right)^{2}$

(ii) expansão transônica

se $u_{i-1}^{n} \leq 0$ e $u_{i}^{n} \geq 0$, temos $g_{i-1 / 2}^{n}=0$.

Se $u_{i-1}^{n} \geq u_{i}^{n}$, então

(iii) choque transônico

se $S=\frac{1}{2}\left(u_{i-1}^{n}+u_{i}^{n}\right)>0$, temos $g_{i-1 / 2}^{n}=\frac{1}{2}\left(u_{i-1}^{n}\right)^{2}$

se $S \leq 0$, temos $g_{i-1 / 2}^{n}=\frac{1}{2}\left(u_{i}^{n}\right)^{2}$.

$g_{i+1 / 2}^{n}$ é definido de maneira similar. A Figura 3.3.6 mostra as características para tais casos.

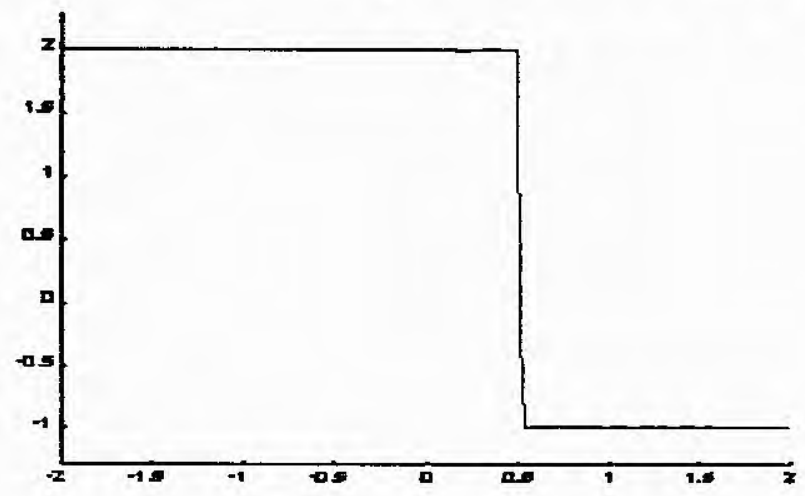

Figura 3.3.5. Solução utilizando o esquema de Godunov para a equação de Burgers com condição inicial, $v_{0}(x)=\left\{\begin{array}{ll}2.0 & \text { se } x \leq 0 \\ -1.0 & \text { se } x>0,\end{array}\right.$ no intervalo $[-2,2]$ e condições de fronteiras $v(-2, t)=2$ e $v(2, t)=-1 \operatorname{com} \Delta x=0.02, \Delta t=0.01$, no tempo $t=1.0$.

Foi verificado anteriormente que problemas de Riemann da forma (3.69)-(3.70) geralmente possuem mais de uma solução. As soluções (3.76) e (3.77) no Exemplo 3.3 .6 são soluções entrópicas dos problemas locais de Riemann e foram escolhidas com o objetivo de obter a solução entrópica do problema que estamos resolvendo. Baseando nas idéias do exemplo anterior, vamos a seguir apresentar a solução entrópica para o problema de Riemann para uma função de fluxo $G$ convexa genérica.

$$
\bar{U}_{t}+[G(\bar{U})]_{x}=0, \quad x \in \mathbb{R}, \quad t>t_{n}
$$




$$
\vec{U}\left(x, t_{n}\right)= \begin{cases}u_{i-1}^{n} & \text { se } x-x_{i-1 / 2}<0 \\ u_{i}^{n} & \text { se } x-x_{i-1 / 2} \geq 0, \quad x \in \mathbb{R}\end{cases}
$$

Esta solução será dada por:

$$
\bar{U}\left(x_{i-1 / 2}, t_{n+1}\right)= \begin{cases}u_{i-1}^{n} & \text { se } G^{\prime}\left(u_{i-1}^{n}\right), G^{\prime}\left(u_{i}^{n}\right) \geq 0 \\ u_{i}^{n} & \text { se } G^{\prime}\left(u_{i-1}^{n}\right), G^{\prime}\left(u_{i}^{n}\right) \leq 0 \\ u_{S} & \text { se } G^{\prime}\left(u_{i-1}^{n}\right)<0<G^{\prime}\left(u_{i}^{n}\right) \\ u_{i-1}^{n} & \text { se } G^{\prime}\left(u_{i-1}^{n}\right) \geq 0 \geq G^{\prime}\left(u_{i}^{n}\right) \text { e } S=a_{i-1 / 2}^{n}>0 \\ u_{i}^{n} & \text { se } G^{\prime}\left(u_{i-1}^{n}\right) \geq 0 \geq G^{\prime}\left(u_{i}^{n}\right) \text { e } S=a_{i-1 / 2}^{n}<0\end{cases}
$$

onde $a_{i-1 / 2}^{n}$ foi definido pela Equação (3.55) e $u_{S}$ é tal que $G^{\prime}\left(u_{S}\right)=0$. $u_{S}$ é o ponto para o qual a velocidade da característica é nula e é chamado de ponto sônico.

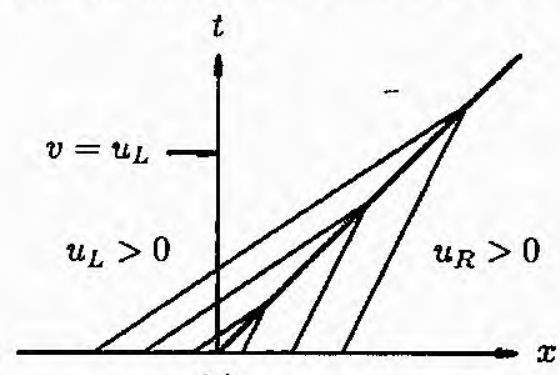

(a)

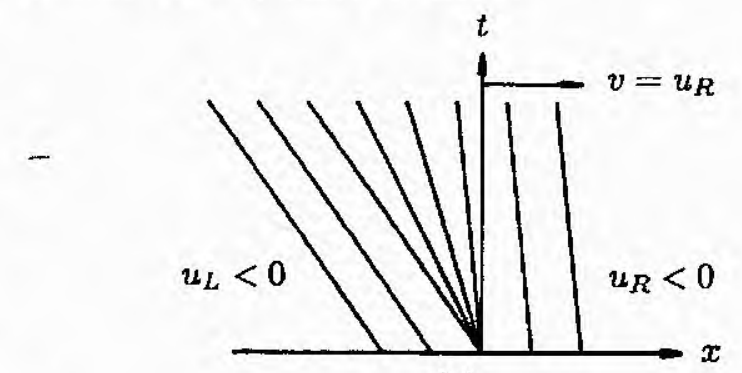

(b)

Características para o caso (i): (a) $u_{R}>u_{L}>0$;

(b) $u_{L}<u_{R}<0$.

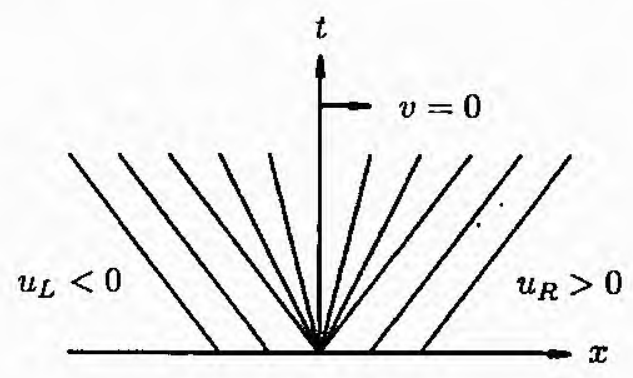

Características para o caso (ii).

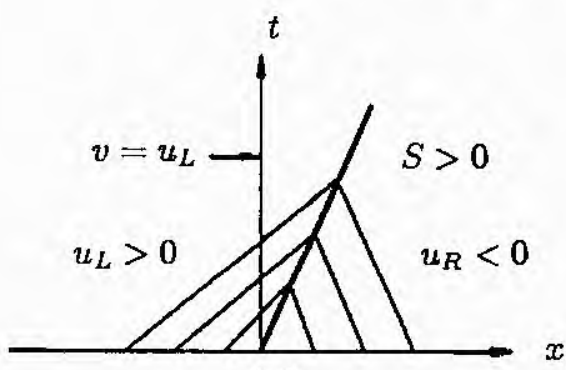

(a)

Características para o caso (iii): (a) $S>0$;

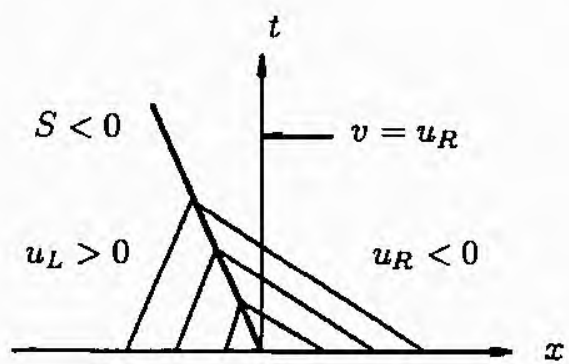

(b)

(b) $S<0$.

Figura 3.3.6. 
Observação 3.3.7. As primeiras duas partes da solução dada em (3.80) correspondem a choques ou rarefações que estão à dirreita e à esquerda da reta $x=x_{i-1 / 2}$ em $t=t_{n+1}$. A terceira e a quarta parte da solução correspondem a choques, onde a localização deste é determinada pela velocidade de propagação do choque S. E finalmente, a quinta parte representa uma rarefação que cruza a reta $x=x_{i-1 / 2}$.

Observaçāo 3.3.8. Podemos escrever a função de fluxo (3.75) usando a solução $\bar{U}$ dada em (3.80) como

$$
g_{i-1 / 2}^{n}= \begin{cases}G_{i-1}^{n} & \text { se } G^{\prime}\left(u_{i-1}^{n}\right), G^{\prime}\left(u_{i}^{n}\right) \geq 0 \\ G_{i}^{n} & \text { se } G^{\prime}\left(u_{i-1}^{n}\right), G^{\prime}\left(u_{i}^{n}\right) \leq 0 \\ G\left(u_{S}\right) & \text { se } G^{\prime}\left(u_{i-1}^{n}\right)<0<G^{\prime}\left(u_{i}^{n}\right) \\ G_{i-1}^{n} & \text { se } G^{\prime}\left(u_{i-1}^{n}\right) \geq 0 \geq G^{\prime}\left(u_{i}^{n}\right) \text { e } S=a_{i-1 / 2}^{n}>0 \\ G_{i}^{n} & \text { se } G^{\prime}\left(u_{i-1}^{n}\right) \geq 0 \geq G^{\prime}\left(u_{i}^{n}\right) \text { e } S=a_{i-1 / 2}^{n}<0 .\end{cases}
$$

O esquema de diferenças (3.74), (3.81) é ùm esquema upwind, conservativo e produz a solução entrópica como já mencionamos. Também pode ser verificado que a função de fluxo numérico associada com o esquema de Godunov pode ser escrita na forma

$$
g_{i+1 / 2}^{\mathrm{G}}= \begin{cases}\min _{u \in\left[u_{i}, u_{i+1}\right]} G(u) & \text { se } u_{i} \leq u_{i+1} \\ \max _{u \in\left[u_{i+1}, u_{i}\right]} G(u) & \text { se } u_{i}>u_{i+1}\end{cases}
$$

Para verificar a validade de (3.82), iremos mostrar, por exemplo, o caso em que $u_{i+1}^{n}<u_{i}^{n}$ baseando-nos em (3.81). O caso $u_{i+1}^{n} \geq u_{i}^{n}$, pode ser verificado analogamente.

Como primeiro (segundo) caso, suponha que $G$ é convexa e estritamente crescente (decrescente) para todo $u \in\left[u_{i+1}, u_{i}\right]$. Temos que $G^{\prime}\left(u_{i}^{n}\right), G^{\prime}\left(u_{i+1}^{n}\right) \geq 0(\leq 0)$ e portanto, $g_{i+1 / 2}^{n}=G\left(u_{i}^{n}\right)\left(G\left(u_{i+1}^{n}\right)\right)=\max _{u_{i+1}^{n} \leq u \leq u_{i}^{n}} G(u)$. Para o terceiro (quarto) caso, suponha que $G$ é convexa e que valha $G^{\prime}\left(u_{i}^{n}\right) \geq 0 \geq G^{\prime}\left(u_{i+1}^{n}\right)$ e $S>0(S<0)$ para todo $u \in\left[u_{i+1}, u_{i}\right]$. Isto implica que $G\left(u_{i+1}^{n}\right)<G\left(u_{i}^{n}\right)\left(G\left(u_{i+1}^{n}\right)>G\left(u_{i}^{n}\right)\right)$ e portanto, $g_{i+1 / 2}^{n}=G\left(u_{i}^{n}\right)\left(G\left(u_{i+1}^{n}\right)\right)=$ $\max _{u_{i+1}^{n} \leq u \leq u_{i}^{n}} G(u)$. No quinto e último caso temos que $G^{\prime}\left(u_{i}^{n}\right)<0<G^{\prime}\left(u_{i+1}^{n}\right)$. Estamos supondo para todos os casos que a função $G$ não possui ponto de inflexão no intervalo $\left[u_{i+1}, u_{i}\right]$. Assim sendo, $G$ terá que ser côncava nesse intervalo e $G^{\prime}\left(u_{S}\right)=0$ para algum $u_{S} \in\left(u_{i+1}, u_{i}\right)$. O ponto $u_{S}$ é conhecido como ponto sônico e é um ponto de máximo de $G$ em $\left(u_{i+1}, u_{i}\right)$, o que implica que $g_{i+1 / 2}^{n}=G\left(u_{S}\right)=\max _{u_{i+1}^{n} \leq u \leq u_{i}^{n}} G(u)$. (Se tivéssemos considerando a condição $u_{i+1}^{n} \geq u_{i}^{n}$ para o quinto caso, onde $G$ não possui ponto de inflexão no intervalo $\left[u_{i}, u_{i+1}\right], G$ seria convexa) 


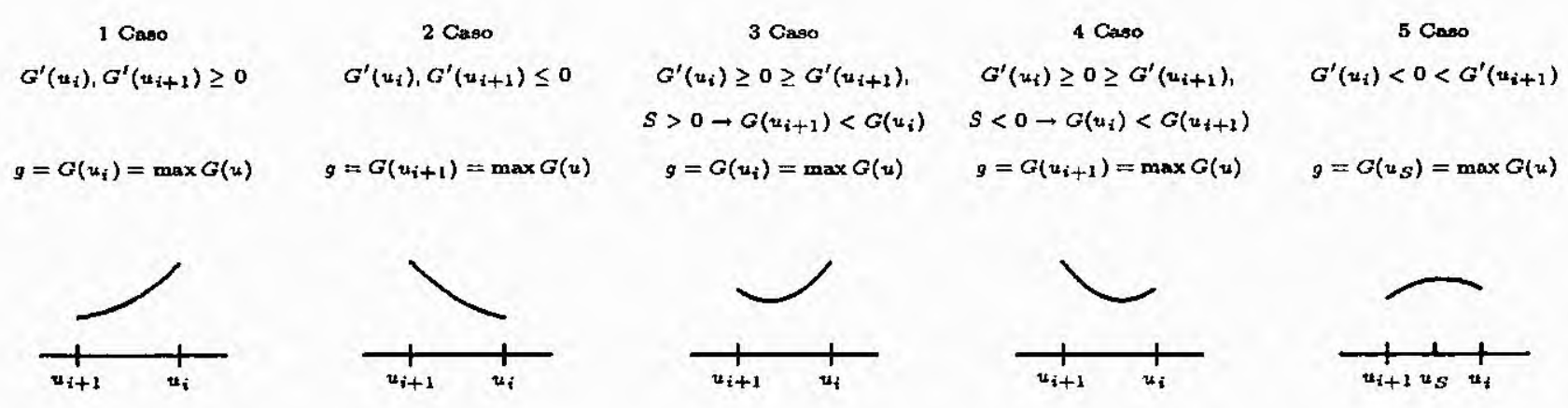

\section{Esquema de Engquist-Osher}

Na seção anterior apresentamos o esquema conservativo de Godunov:

$$
u_{i}^{n+1}=u_{i}^{n}-R\left[G \left(\widetilde{U}\left(x_{i+1 / 2}, t\right)-G\left(\bar{U}\left(x_{i-1 / 2}, t\right)\right]\right.\right.
$$

em que $\breve{U}$ é a solução para o problema de Riemann com valores $u_{i}^{n}$ a esquerda e $u_{i+1}^{n}$ a direita da descontinuidade. O método de Godunov, como já salientamos, possui propriedades desejáveis de um método numérico para leis de conservação, no entanto, como também já dissemos, este método possui uma dificuldade intrínsica que é a necessidade de solução de problemas de Riemann a todo passo de tempo. Gostaríamos de encontrar um esquema que conserve as propriedades upwind, quando $a \neq 0$, tenha pouca difusividade, produza a solução entrópica e seja mais simples de usar que o método de Godunov.

Um método que satisfaz os requisitos acima é o de Engquist-Osher, dado por:

$$
u_{i}^{n+1}=u_{i}-R\left\{G\left(u_{i}\right)-G\left(u_{i-1}\right)+\int_{u_{i}}^{u_{i+1}} \min (a(u), 0) d u-\int_{u_{i-1}}^{u_{i}} \min (a(u), 0) d u\right\},
$$

em que $a(u)=\frac{d G}{d u}$ é a velocidade de propagação. Sua função de fluxo numérico, $g^{\mathrm{EO}}$, é dada por:

$$
g^{\mathrm{EO}}\left(u_{L}, u_{R}\right)=G\left(u_{L}\right)+\int_{u_{L}}^{u_{R}} \min (a(u), 0) d u
$$

De (3.84) vemos que $g^{\mathrm{EO}}$ é a função de fluxo de Godunov mais um termo difusivo. A notação $g^{G}$ será utilizada para representar a função de fluxo numérico de Godunov. No caso em que $G$ é uma função estritamente convexa, isto é, $\left(G^{\prime \prime}>0\right)$, podemos comparar a função de fluxo $g^{\mathrm{EO}}$ com $g^{\mathrm{C}}$, (veja também Figura 3.3.7): 
(1) Se $a(u)>0$, $u$ entre $u_{L}$ e $u_{R}$ : A onda se moverá para a direita e portanto, $g^{\mathrm{EO}}=g^{\mathrm{G}}=$ $G\left(u_{L}\right)$.

(2) Se $a(u)<0, u$ entre $u_{L}$ e $u_{R}$ : A onda se moverá para a esquerda e portanto, $g^{\mathrm{RO}}=g^{\mathrm{G}}=$ $G\left(u_{R}\right)$.

(3) Se $a\left(u_{L}\right)<0<a\left(u_{R}\right)$ : Uma rarefação surge e portanto, $g^{\mathrm{EO}}=g^{\mathrm{G}}=G\left(a^{-1}(0)\right)=G\left(u_{S}\right)$, e assim o esquema escolhe a imagem inversa do zero.

(4) Se $a\left(u_{L}\right)>0>a\left(u_{R}\right)$ : Um choque desenvolve-se.

O caso 4, que representa o caso transonico, precisa ser melhor analisado pois $g^{\mathbf{G}}=G\left(u_{L}\right)$ ou $g^{G}=G\left(u_{R}\right)$, que neste caso é a solução exata, depende do sinal de $S$ que é derivado da condição de Rankine-Hugoniot. Entretanto $g^{\mathrm{EO}}$ é dada por:

$$
\begin{aligned}
g^{\mathrm{EO}}\left(u_{L}, u_{R}\right) & =G\left(u_{L}\right)+\int_{u_{L}}^{u_{R}} \min (a(u), 0), d u \\
& =G\left(u_{L}\right)+\int_{u_{L}}^{a^{-1}(0)} \min (a(u), 0), d u+\int_{a^{-1}(0)}^{u_{R}} \min (a(u), 0), d u \\
& =G\left(u_{L}\right)+G\left(u_{R}\right)-G\left(u_{S}\right) .
\end{aligned}
$$

A primeira integral na direita da igualdade é zero, pois a velocidade da onda é positiva de $u_{L}$ até a esquerda de $a^{-1}(0)$. Portanto para este caso constatamos que $g^{\mathrm{EO}} \neq g^{\mathrm{C}}$ e que o esquema de Engquist-Osher é um pouco mais difusivo do que o de Godunov. Por exemplo, para a equação de Burgers, $G(v)=v^{2} / 2$, temos que

$$
g^{\mathrm{G}}=\left\{\begin{array}{lll}
\frac{u_{L}^{2}}{2} & \ldots & \text { se } \ldots S>0 \\
\frac{u_{K}^{2}}{2} & \ldots & \text { se } \ldots S<0
\end{array}\right.
$$

e

$$
g^{\mathrm{EO}}=\frac{u_{L}^{2}}{2}+\frac{u_{R}^{2}}{2}
$$
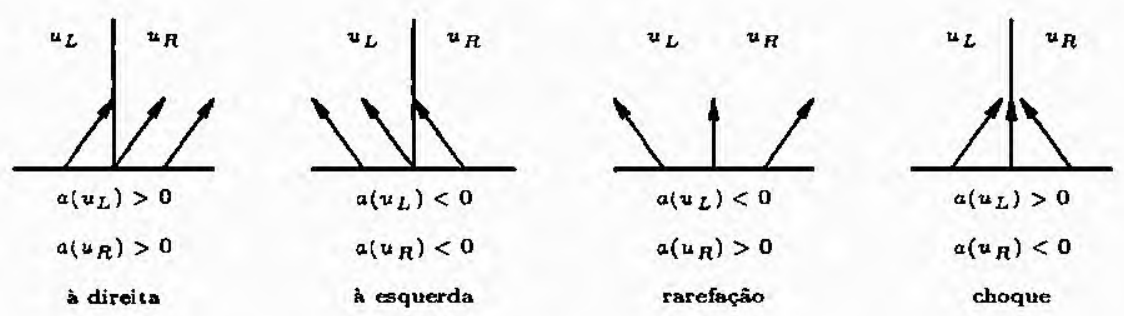

Figura 3.3.7. Possíveis soluções para o problema local de Riemann.

Notamos que a solução numérica produzida por este esquema é mais difusiva do que a solução de Godunov. Portanto. a solução numérica em torno das descontinuidades é 
suavizada, mas somente para o caso de fluxo transônico. Assim sendo, todos os quatros casos são bem escolhidos; três deles fornece a solução exata e o quarto acrescenta um pouco de difusão à solução exata.

$\mathrm{Na}$ referência [29]; os autores derivam um esquema com as mesmas propriedades do esquema de Engquist-Osher, para o caso particular da equação da onda não linear $v_{t}+\left[v^{2}\right]_{x}=$ 0 , a saber,

$$
g\left(u_{L}, u_{R}\right)=\left(\max \left\{u_{L}, 0\right\}^{2}+\min \left\{u_{R}, 0\right\}^{2}\right) .
$$

Esse esquema será utilizado quando formos aproximar os gradientes para ambos os problemas de valor inicial e de fronteira, pois é um esquema que satisfaz a condição de entropia, com relativamente pouca difusão.

\subsubsection{Esquemas de Segunda Ordem de Lax-Wendroff e de Beam- Warming}

Nesta seção apresentamos os métodos de segunda ordem de Lax-Wendroff e de BeamWarming para os casos linear e não linear, respectivamente. Para o caso linear a equação $v_{t}+a v_{x}=0$ pode ser escrita como $v_{t}=-a v_{x}$, e ainda

$$
v_{t t}=\left(-a v_{x}\right)_{t}=-a v_{x t}=-a\left(v_{t}\right)_{x}=-a\left(-a v_{x}\right)_{x}=a^{2} v_{x x}
$$

\section{Lax-Wendroff:}

Seja $v(x, t)$ uma solução diferenciável da equação linear acima, a expansão em série de Taylor produz

$$
v(x, t+\Delta t)=v(x, t)+\Delta t v_{t}(x, t)+\frac{\Delta t^{2}}{2} v_{t t}(x, t)+o\left(\Delta t^{3}\right)
$$

Rescrevendo (3.86), temos

$$
\begin{aligned}
v_{i}^{n+1} & =v_{i}^{n}+\Delta t\left(v_{t}\right)_{i}^{n}+\frac{\Delta t^{2}}{2}\left(v_{t t}\right)_{i}^{n}+o\left(\Delta t^{3}\right) \\
& =v_{i}^{n}+\Delta t\left(-a v_{x}\right)_{i}^{n}+\frac{\Delta t^{2}}{2}\left(a^{2} v_{x x}\right)_{i}^{n}+o\left(\Delta t^{3}\right) \\
& =v_{i}^{n}-a \Delta t\left(\frac{v_{i+1}^{n}-v_{i-1}^{n}}{2 \Delta x}+o\left(\Delta x^{2}\right)\right)+\frac{(a \Delta t)^{2}}{2}\left(\frac{v_{i+1}^{n}-2 v_{i}^{n}+v_{i-1}^{n}}{\Delta x^{2}}+o\left(\Delta x^{2}\right)\right)+o\left(\Delta t^{3}\right) \\
& =v_{i}^{n}-\frac{a \Delta t}{2 \Delta x} \delta_{0} v_{i}^{n}+\frac{a^{2} \Delta t^{2}}{2 \Delta x^{2}} \delta^{2} v_{i}^{n}+o\left(\Delta t \Delta x^{2}\right)+o\left(\Delta t^{3}\right)
\end{aligned}
$$


e portanto aproximamos a equação diferencial parcial $v_{t}+a v_{x}=0$ pelo esquema de diferenças

$$
u_{i}^{n+1}=u_{i}^{n}-\frac{\lambda}{2} \delta_{0} u_{i}^{n}+\frac{\lambda^{2}}{2} \delta^{2} u_{i}^{n}
$$

em que $\lambda=a R$. O esquema de diferenças (3.88) é chamado de esquema de Lax-Wendroff é é obviamente de $o\left(\Delta x^{2}\right)+o\left(\Delta t^{2}\right)$ e estável para $|\lambda| \leq 1$.

Notações 3.3.9. Definiremos os seguintes operadores:

$$
\delta_{+} u_{i}^{n}=u_{i+1}^{n}-u_{i}^{n}, \delta_{-} u_{i}^{n}=u_{i}^{n}-u_{i-1}^{n}, \delta_{0} u_{i}^{n}=u_{i+1}^{n}-u_{i-1}^{n}, \delta^{2} u_{i}^{n}=u_{i+1}^{n}-2 u_{i}^{n}+u_{i-1}^{n} .
$$

Para o caso não linear, considere a equação diferencial parcial na forma conservativa $v_{t}=-\frac{\partial}{\partial x} G(v)$. Fazendo as substituiçôes convenientes em (3.86), temos

$$
v(x, t+\Delta t)=v(x, t)-\Delta t \frac{\partial G(x, t)}{\partial x}-\frac{\Delta t^{2}}{2} \frac{\partial}{\partial t}\left(\frac{\partial G(v(x, t))}{\partial x}\right)+o\left(\Delta t^{3}\right) .
$$

Utilizando as aproximações mencionadas anteriormente. e que

$$
\frac{\partial}{\partial t}\left(\frac{\partial G}{\partial x}\right)=\frac{\partial}{\partial x}\left(\frac{\partial G}{\partial t}\right)=-\frac{\partial}{\partial x}\left(\frac{\partial G}{\partial v} \frac{\partial G}{\partial x}\right)
$$

temos o esquema de Lax-Wendroff para a lei de conservação não linear, dado por

$$
u_{i}^{n+1}=u_{i}-\frac{R}{2}\left\{G_{i+1}-G_{i-1}\right\}+\frac{R^{2}}{2}\left[\left(G^{\prime}(u)\right)_{i+1 / 2}^{n}\left(G_{i+1}-G_{i}\right)-\left(G^{\prime}(u)\right)_{i-1 / 2}^{n}\left(G_{i}-G_{i-1}\right)\right],
$$

$\operatorname{com} G_{i+1}=G\left(u_{i+1}^{n}\right), \quad G_{i}=G\left(u_{i}^{n}\right), \quad G_{i-1}=G\left(u_{i-1}^{n}\right)$,

$$
\left\{\begin{array}{l}
\left(G^{\prime}(u)\right)_{i+1 / 2}^{n} \approx G^{\prime}\left(\frac{u_{i-1}^{n}+u_{i}^{n}}{2}\right) . \\
\left(G^{\prime}(u)\right)_{i-1 / 2}^{n} \approx G^{\prime}\left(\frac{u_{i}^{n}+u_{i-1}^{n}}{2}\right),
\end{array}\right.
$$

O esquema (3.90) é de $o\left(\Delta x^{2}\right)+o\left(\Delta t^{2}\right)$ e pode ser escrito na forma conservativa com função de fluxo numérico dada por

$$
g^{\mathrm{LW}}(u, v)=\frac{G(u)+G(v)}{2}-\frac{\lambda}{2} G^{\prime}\left(\frac{u+v}{2}\right)(G(v)-G(u)) .
$$

Outra forma para o esquema de Lax-Wendroff que é ligeiramente diferente de (3.90) (diferente quando $u_{i}^{n}=u_{i \pm 1}^{n}$ ) é:

$$
u_{i}^{n+1}=u_{i}-\frac{R}{2} \delta_{0} G_{i}^{n}+\frac{R^{2}}{2} \delta_{-}\left\{\left(a_{i+1 / 2}^{n}\right)^{2} \delta_{+} G_{i}^{n}\right\}
$$


$\operatorname{com} a_{i+1 / 2}^{n}$ dado em (3.55).

\section{Beam-Warming:}

A versão linear do esquema de Beam-Warming é derivada da seguinte maneira: Considere a equaçāo (3.87) aproximada da seguinte forma

$$
\begin{aligned}
v_{i}^{n+1}= & v(x, t)-a k\left(\frac{v(x, t)-v(x-h, t)}{h}+\frac{h}{2} v_{x x}(x, t)+o\left(h^{2}\right)\right)_{i}^{n}+\frac{(a k)^{2}}{2}\left(v_{x x}\right)_{i}^{n}+o\left(k^{3}\right) \\
= & v-a k\left(\frac{v(x, t)-v(x-h, t)}{h}+\frac{h}{2}\left(\frac{v(x, t)-2 v(x-h, t)+v(x-2 h, t)}{h^{2}}\right)+o\left(h^{2}\right)\right)_{i}^{n} \\
& +\frac{(a k)^{2}}{2}\left(\frac{v(x, t)-v(x-h, t)+v(x-2 h, t)}{h^{2}}+o(h)\right)_{i}^{n}+o\left(k^{3}\right) .
\end{aligned}
$$

Assim sendo, obtemos a aproximação para a equaçào diferencial parcial $v_{t}+a v_{x}=0$ por meio do esquema de diferenças

$$
\begin{aligned}
u_{i}^{n+1} & =u_{i}^{n}-\lambda \delta_{-} u_{i}^{n}-\frac{\lambda}{2}\left(\delta_{-}\right)^{2} u_{i}^{n}+\frac{\lambda^{2}}{2}\left(\delta_{-} u_{i}^{n}-\delta_{-} u_{i-1}^{n}\right) \\
& =\frac{1}{2}\left[u_{i}^{n}+u_{i}^{*}-\lambda \delta_{-} u_{i}^{*}-\lambda\left(\delta_{-}\right)^{2} u_{i}^{n}\right]
\end{aligned}
$$

onde $u_{i}^{*}=u_{i}^{n}-\lambda \delta_{-} u_{i}^{n}$. Esse esquema explícito é estável para $0 \leq \lambda \leq 2$ e possui ordem $o\left(\Delta t^{2}\right)+o(\Delta t \Delta x)+o\left(\Delta x^{2}\right)$.

A versão não linear do esquema de Beam-Warming é obtida considerando a seguinte discretizaçāo da equação (3.89):

$$
\begin{aligned}
v_{i}^{n+1}= & v_{i}^{n}-k\left(\frac{G_{i}^{n}-G_{i-1}^{n}}{h}+\frac{1}{2} \frac{G_{i}^{n}-2 G_{i-1}^{n}+G_{i-2}^{n}}{h}+o\left(h^{2}\right)\right)-\frac{k^{2}}{2 h^{2}}\left[G^{\prime}\left(v_{i}^{n}\right)\left(\delta_{-} G_{i}^{n}+o(h)\right)\right. \\
& \left.-G^{\prime}\left(v_{i-1}^{n}\right)\left(\delta_{-} G_{i-1}^{n}+o(h)\right)+o(h)\right]+o\left(k^{3}\right) .
\end{aligned}
$$

Aproximando a equaçāo (3.95), temos

$$
\begin{aligned}
u_{i}^{n+1}= & u_{i}^{n}-\frac{R}{2}\left(G_{i}^{n}-G_{i-1}^{n}\right)-\frac{R}{2}\left(G_{i}^{n}-G_{i-1}^{n}\right)-\frac{R}{2}\left(\delta_{-}\right)^{2} G_{i}^{n} \\
& -\frac{R^{2}}{2}\left[G^{\prime}\left(u_{i}^{n}\right)\left(G_{i}^{n}-G_{i-1}^{n}\right)-G^{\prime}\left(u_{i-1}^{n}\right)\left(G_{i-1}^{n}-G_{i-2}^{n}\right)\right] \\
= & u_{i}^{n}-\frac{R}{2}\left(G_{i}^{n}-G_{i-1}^{n}\right)-\frac{R}{2}\left[G_{i}^{n}-R\left(G^{\prime}\right)_{i}^{n} \delta_{-} G_{i}^{n}-G_{i-1}^{n}+R\left(G^{\prime}\right)_{i-1}^{n} \delta_{-} G_{i-1}^{n}\right]-\frac{R}{2}\left(\delta_{-}\right)^{2} G_{i}^{n} \\
= & u_{i}^{n}-\frac{R}{2}\left(G_{i}^{n}-G_{i-1}^{n}\right)-\frac{R}{2}\left[G\left(u_{i}^{n}-R \delta_{-} G_{i}^{n}\right)-G\left(u_{i-1}^{n}-R \delta_{-} G_{i-1}^{n}\right]-\frac{R}{2}\left(\delta_{-}\right)^{2} G_{i}^{n}\right. \\
= & \frac{1}{2}\left[u_{i}^{n}+u_{i}^{*}\right]-\frac{R}{2} \delta_{-} G_{i}^{*}-\frac{R}{2}\left(\delta_{-}\right)^{2} G_{i}^{n} .
\end{aligned}
$$


em que $u_{i}^{*}=u_{i}^{n}-R \delta_{-} G_{i}^{n}$. O termo $-\frac{R}{2}\left(\delta_{-}\right)^{2} G_{i}^{n}$ faz com que esse esquema seja de segunda ordem de precisão. Ambos os esquemas mencionados nesta seção serão usados mais à frente para derivarmos esquemas de alta resolução.
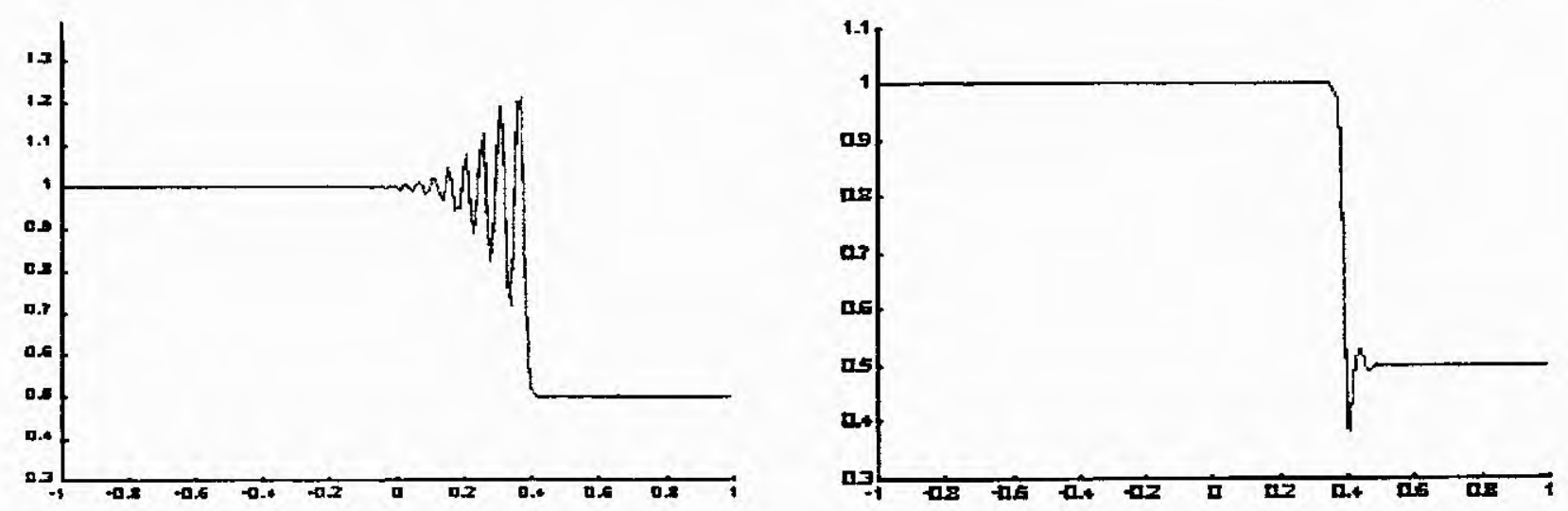

Figura 3.3.8. Solução utilizando os esquemas de Lax-Wendroff e de Beam-Warming, para a equação de Burgers com condição inicial (3.97), $\Delta x=0.01, \Delta t=0.001$ no tempo $t=0.5$.

Exemplo 3.3.10. Neste exemplo usamos os esquemas de Lax-Wendroff (3.90) e de BeamWarming (3.96) para aproximarmos a equação invíscida de Burgers com condição inicial

$$
v_{0}(x)=v(x, 0)= \begin{cases}1.0 & \text { se } x \leq 0 \\ 0.5 & \text { se } x>0\end{cases}
$$

no intervalo $[-1,1]$ com condições de fronteira numéricas $u_{0}^{n}=1, u_{M}^{n}=0.5$.

Como observamos através das Figura 3.3.8, os métodos de Lax-Wendroff e de BeamWarming introduzem oscilações no entorno das descontinuidades. Para eliminar essas oscilações indesejáveis, presisaremos forçar esses métodos a preservar monotonicidade. Isso é o que veremos daqui por diante.

\subsubsection{Definições e Resultados}

Consideremos novamente a lei de conservação escalar

$$
v_{t}+G(v)_{x}=0
$$

Começamos introduzindo uma classe de esquemas de diferenças chamados esquemas de entropia, ou esquemas-E, veja [28]. A busca de esquemas munidos das propriedades de entropia, monotonicidade e TVD, definidas logo a seguir, justifica-se pelo fato de esses esquemas convergirem para a solução entrópica do problema de valor inicial (3.56)-(3.57). 
Dessa maneira, iniciaremos fornecendo uma receita teórica para construir esquemas que convegem para a solução entrópica.

Definição 3.3.11. Definimos um esquema numérico como sendo um esquema-E se:

$$
\operatorname{sgn}\left(u_{i+1}-u_{i}\right)\left[g_{i+1 / 2}-G(u)\right] \leq 0
$$

para todo $u$ entre $u_{i}$ e $u_{i+1}$, onde

$$
\operatorname{sgn}(x)= \begin{cases}1 & \text { se } x>0 \\ -1 & \text { se } x<0\end{cases}
$$

A seguir, forneceremos uma importante caracterizaçào para esquemas-E relacionando-os com a função de fluxo numérico do esquema de Godunov.

Lema 3.3.12. Assumindo que (3.61) é verdadeira, isto é, $C F L \leq 1$, então as funções de fluxo numérico de esquemas-E são caracterizadas por

$$
\begin{cases}g_{i+1 / 2}^{n} \leq g_{i+1 / 2}^{G} & \text { se } u_{i}^{n}<u_{i+1}^{n} \\ g_{i+1 / 2}^{n} \geq g_{i+1 / 2}^{G} & \text { se } u_{i}^{n}>u_{i+1}^{n}\end{cases}
$$

em que $g^{G}$ representa a função de fluxo numérico do esquema de Godunov.

Demonstração: Para $u_{i}^{n}<u_{i+1}^{n}$, temos da equação (3.82), que $g_{i+1 / 2}^{\mathrm{G}}=\min _{u_{i}^{n} \leq u \leq u_{i+1}^{n}} G(u)$. Assim $u_{i+1}^{n}-u_{i}^{n}>0$ e portanto $g_{i+1 / 2}^{n}<G(u), \forall u \in\left[u_{i}, u_{i+1}\right]$. Dessa maneira, concluímos que $g_{i+1 / 2}^{n} \leq \min _{u_{i}^{n} \leq u \leq u_{i+1}^{n}} G(u)=g_{i+1 / 2}^{\mathrm{G}}$. Para $u_{i}^{n}>u_{i+1}^{n}$ temos que $g_{i+1 / 2}^{G}=\max _{u_{i+1}^{n} \leq u \leq u_{i}^{n}} G(u)$. Assim $u_{i+1}^{n}-u_{i}^{n}<0$ e portanto $g_{i+1 / 2}^{n}>G(u), \forall u \in\left[u_{i+1}, u_{i}\right]$. Dessa maneira, concluímos que $g_{i+1 / 2}^{n}>\max _{u_{i+1}^{n} \leq u \leq u_{i}^{n}} G(u)=g_{i+1 / 2}^{G}$.

Observaçāo 3.3.13. O esquema FTFS é um esquema-E.

Demonstração: Para o esquema FTFS, $g_{i+1 / 2}^{n}=G_{i+1}^{n}=G\left(u_{i+1}^{n}\right)$. A condição CFL para esse esquema é $-1 \leq R G^{\prime} \leq 0$ e assim $G^{\prime}$ é negativa e portanto $G$ é decrescente. Então se $u_{i}<u_{i+1}$, para $u \in\left[u_{i}, u_{i+1}\right]$, temos $g\left(u_{i}, u_{i+1}\right)=G\left(u_{i+1}\right) \leq G(u)$. Se $u_{i+1}<u_{i}$, para $u \in\left[u_{i+1}, u_{i}\right]$, temos $g\left(u_{i}, u_{i+1}\right)=G\left(u_{i+1}\right) \geq G(u)$. Portanto, o esquema FTFS é um esquema-E.

Geralmente, não é simples decidir se um determinado esquema é ou nào um esquema-E. Uma definição mais simples, torna essa decisão também mais simples. No entanto, devemos certificar que as propriedades dos esquemas respeitando essa definição sejam boas o suficiente 
para garantir, por exemplo, a aproximação da solução entrópica. Os métodos monótonos satisfazem esses requisitos, e ainda mais, o fato de um esquema numérico não ser monótono está associado com o fenômeno de oscilações espúrias nas proximidades de altos gradientes.

Definição 3.3.14. Um esquema de diferenças na forma (3.64) é dito ser monótono se a função $W$ é uma função crescente monótona com respeito a cada um de seus argumentos.

Observação 3.3.15. Se $W$ for diferenciável com respeito a cada um de seus argumentos, então o esquema de diferenças (3.64) é monótono se e somente se

$$
\frac{\partial W\left(u_{-(p+1)}, \ldots, u_{q}\right)}{\partial u_{j}} \geq 0 \text { para todo } j,-(p+1) \leq j \leq q \text {. }
$$

Exemplo 3.3.16. Um exemplo de um esquema monótono é o esquema de primeira ordem de Lax-Friedrichs. Esse esquema é construído através de diferenças centrais para aproximar $G(v)_{x}$ e é dado por

$$
u_{i}^{n+1}=\frac{1}{2}\left[u_{i-1}^{n}+u_{i+1}^{n}\right]-\frac{k}{2 h}\left[G_{i+1}-G_{i-1}\right]
$$

Ele pode ser colocado na forma conservativa por meio da função de fluxo numérico

$$
g^{L F}\left(u_{i}^{n}, u_{i+1}^{n}\right)=-\frac{h}{2 k}\left(u_{i+1}^{n}-u_{i}^{n}\right)+\frac{1}{2}\left[G\left(u_{i+1}^{n}\right)+G\left(u_{i}^{n}\right)\right] .
$$

$O$ esquema de Lax-Friedrichs é monótono se

$$
\begin{gathered}
\partial W / \partial u_{-1}\left(u_{-1}, u_{0}, u_{1}\right)=1 / 2+R G^{\prime}\left(u_{-1}\right) / 2 \geq 0 \\
\partial W / \partial u_{1}\left(u_{-1}, u_{0}, u_{1}\right)=1 / 2-R G^{\prime}\left(u_{1}\right) / 2 \geq 0
\end{gathered}
$$

que é assegurado pela condição $C F L, R \max \left|G^{\prime}(u)\right| \leq 1$.

Uma vantagem do esquema de Lax-Friedrichs é que não precisamos saber quase nada sobre a estrutura da função de fluxo $G$ e, uma desvantagem, é que ele introduz difusão na solução, ou seja, descontinuidades são suavizadas, e portanto, frentes não permanecem aguçadas. $O$ método de Godunov apresentado anteriormente é menos difusivo do que o de Lax-Friedrichs e por isso é mais utilizado pois transporta singularidades sem deformá-las, reproduzindo mais fielmente uma frente em movimento. Ambos os métodos serão mencionados posteriormente.

Um resultado muito importante que agora podemos enunciar, mas que não provaremos (veja [15], pag. 144) é, Um esquema conservativo, monótono produz uma solução que satisfaz a condição de entropia. 
Prosseguindo com nossas definições, definiremos uma outra classe de esquemas de discretização para uma lei de conservação com propriedades adequadas para evitar o aparecimento de oscilações na solução, cuja propriedade principal deles será de amortecer essas oscilaçōes.

Definição 3.3.17. Um esquema de diferenças é dito ter variação total decrescente (TVD) se a solução produzida por esse esquema satisfaz

$$
\sum_{i=-\infty}^{\infty}\left|\delta_{+} u_{i}^{n+1}\right| \leq \sum_{i=-\infty}^{\infty}\left|\delta_{+} u_{i}^{n}\right|
$$

para todo $n \geq 0$. Definiremos a variação total por

$$
T V(\mathbf{u})=\sum_{i=-\infty}^{\infty}\left|\delta_{+} u_{i}\right| .
$$

A desigualdade da definição 3.3 .17 pode ser expressa como $T V\left(\mathbf{u}^{n+1}\right) \leq T V\left(\mathbf{u}^{n}\right) \forall n$. Se considerarmos novamente o Exemplo 3.3.10, no primeiro passo $t=\Delta t$, obtemos a solução dada na Tabela 3.3.18. É fácil verificar através da tabela abaixo que,

\begin{tabular}{|c|c|c|c|c|c|c|c|c|c|}
\hline & $u_{0}^{n}$ & $u_{1}^{n}$ & $u_{2}^{n}$ & $u_{3}^{n}$ & $u_{4}^{n}$ & $u_{5}^{n}$ & $u_{6}^{n}$ & $u_{7}^{n}$ & $u_{8}^{n}$ \\
\hline$n=0$ & 1.0 & 1.0 & 1.0 & 1.0 & 1.0 & 0.5 & 0.5 & 0.5 & 0.5 \\
\hline$n=1$ & 1.0 & 1.0 & 1.0 & 1.0 & 1.0623 & 0.7002 & 0.5 & 0.5 & 0.5 \\
\hline
\end{tabular}

Tabela 3.3.18. Valores da condição inicial e da solução em $t=\Delta t$ para o problema resolvido no Exemplo 3.3 .10 com o esquema de Lax-Wendroff (3.90) com $\Delta x=0.25 e$ $\Delta t=0.124$.

$$
T V\left(\mathbf{u}^{0}\right)=\sum_{i=0}^{8}\left|\delta_{+} u_{i}^{0}\right|=0.5
$$

enquanto que

$$
T V\left(\mathbf{u}^{1}\right)=\sum_{i=0}^{8}\left|\delta_{+} u_{i}^{1}\right|=0.6246 .
$$

Portanto, o esquema de Lax-Wendroff (3.90) não é TVD. A seguir, mostraremos utilizando a definição que o esquema FTBS para a equação da onda linear com $a>0$ é TVD.

Demonstração: Seja

$$
u_{i}^{n+1}=u_{i}^{n}-a R \delta_{-} u_{i}^{n} .
$$

- esquema FTBS para a equação da onda linear. Esse esquema é estável para $a>0$, e assumindo que a condiçào CFL, $0 \leq a R \leq 1$ seja satisfeita. temos 
$T V\left(\mathbf{u}^{n+1}\right)=\sum_{i=-\infty}^{\infty}\left|\delta_{+} u_{i}^{n+1}\right|=\sum_{i=-\infty}^{\infty}\left|\delta_{+} u_{i}^{n}-a R \delta_{+} \delta_{-} u_{i}^{n}\right|=\sum_{i=-\infty}^{\infty}\left|\delta_{+} u_{i}^{n}-a R \delta_{-} \delta_{+} u_{i}^{n}\right|=\sum_{i=-\infty}^{\infty} \mid(1-$ $a R) \delta_{+} u_{i}^{n}+a R \delta_{+} u_{i-1}^{n} \mid$ (usando a desigualdade triangular nessa última soma e que $1-a R \geq$ $0, a R \geq 0$, temos)

$\leq(1-a R) \sum_{i=-\infty}^{\infty}\left|\delta_{+} u_{i}^{n}\right|+a R \sum_{i=-\infty}^{\infty}\left|\delta_{+} u_{i-1}^{n}\right|=\sum_{i=-\infty}^{\infty}\left|\delta_{+} u_{i}^{n}\right|-a R \sum_{i=-\infty}^{\infty}\left|\delta_{+} u_{i}^{n}\right|+a R \sum_{i=-\infty}^{\infty}$ $\left|\delta_{+} u_{i-1}^{n}\right|=\sum_{i=-\infty}^{\infty}\left|\delta_{+} u_{i}^{n}\right|=T V\left(\mathbf{u}^{\mathbf{n}}\right)$ (para $j=i-1$ na segunda soma).

Em alguns casos, a derivação de esquemas TVD é muito complicado. Por esta razão, poderemos desenvolver esquemas que relaxam a exigência TVD. Assim sendo, definiremos agora uma classe de esquemas menos restritivo do que os TVD, objetivando obter maior precisão.

Definição 3.3.19. Um esquema de diferenças é dito ser essencialmente não-oscilatório (ENO) se a solução produzida pelo esquema satisfaz

$$
\sum_{i=-\infty}^{\infty}\left|\delta_{+} u_{i}^{n+1}\right| \leq \sum_{i=-\infty}^{\infty}\left|\delta_{+} u_{i}^{n}\right|+o\left(\Delta x^{p}\right)
$$

para todo $n \geq 0$ e algum $p$.

Note que a definição acima relaxa a condição TVD, mas à medida que $\Delta x \rightarrow 0$ o esquema numérico aproxima-se mais e mais de um TVD. No Capítulo 4 apresentaremos brevemente o conceito de interpolação essencialmente não-oscilatória para derivarmos esquemas de segunda ordem para os problemas de valor inicial e de fronteira.

\subsubsection{Forma Incremental e Viscosidade Numérica}

Na prática é muito difícil verificar se um dado esquema é de Entropia, TVD ou ENO pelas definições, e por esta razão, incluimos a seguinte definição que é uma forma mais adequada para estudar as características de esquemas de Entropia e TVD, e com o auxilio de alguns teoremas, poderão facilitar tais verificações.

Definição 3.3.20. Dizemos que o esquema (3.64) pode ser posto na forma incremental ou forma-I se existirem duas funções de $q+p+2$ variáveis, $C$ e $D$, chamadas de coeficientes incrementais, em que

$$
\begin{aligned}
& C_{i+1 / 2}^{n}=C\left(u_{i-p-1}^{n}, \ldots, u_{i+q}^{n}\right) \\
& D_{i+1 / 2}^{n}=D\left(u_{i-p-1}^{n}, \ldots, u_{i+q}^{n}\right)
\end{aligned}
$$


tal que o esquema de diferenças pode ser escrito na forma

$$
u_{i}^{n+1}=u_{i}^{n}+C_{i+1 / 2}^{n} \delta_{+} u_{i}^{n}-D_{i-1 / 2}^{n} \delta_{-} u_{i}^{n}
$$

Proposição 3.3.21. Qualquer esquema conservativo de 3-pontos, isto é, com $p+q+2=3$ em (3.64), consistente com (3.98), com função de fiuxo numérico g, admite uma única forma incremental com coeficientes incrementais dados por

$$
\begin{aligned}
& C_{i+1 / 2}^{n}=-R \frac{g_{i+1 / 2}^{n}-G\left(u_{i}^{n}\right)}{\delta_{+} u_{i}^{n}} \\
& D_{i+1 / 2}^{n}=-R \frac{g_{i+1 / 2}^{n}-G\left(u_{i+1}^{n}\right)}{\delta_{+} u_{i}^{n}} .
\end{aligned}
$$

Demonstração: Considere um esquema conservativo de 3-pontos escrito na forma incremental. Igualando as equações (3.105) e (3.65) temos,

$$
u_{i}^{n}+C_{i+1 / 2}^{n} \delta_{+} u_{i}^{n}-D_{i-1 / 2}^{n} \delta_{-} u_{i}^{n}=u_{i}^{n}-R\left[g_{i+1 / 2}^{n}-g_{i-1 / 2}^{n}\right]
$$

Tomando $u_{i-1}=u_{i}$ temos

$$
C_{i+1 / 2}=C\left(u_{i}, u_{i+1}\right)=-R\left[g\left(u_{i}, u_{i+1}\right)-g\left(u_{i}, u_{i}\right)\right] / \delta_{+} u_{i}^{n}
$$

que munido com a condição de consistência, $g\left(u_{i}, u_{i}\right)=G\left(u_{i}\right)$, produz (3.106). Analogamente, tomando $u_{i}=u_{i+1}$, temos (3.107).

Notemos que esses coeficientes satisfazem a seguinte relação de consistência

$$
D_{i+1 / 2}^{n}-C_{i+1 / 2}^{n}=R a_{i+1 / 2}^{n}
$$

onde $a_{i+1 / 2}^{n}$ foi definido em (3.55). Inversamente, qualquer esquema incremental de 3-pontos pode ser posto na forma conservativa determinada por (3.108), onde a função de fluxo numérico associada com tal esquema pode ser escrita em termos de $C$ e $D$ como

$$
g_{i+1 / 2}^{n}=G\left(u_{i}^{n}\right)-\frac{1}{R} C_{i+1 / 2}^{n} \delta_{+} u_{i}^{n}=G\left(u_{i+1}^{n}\right)-\frac{1}{R} D_{i+1 / 2}^{n} \delta_{+} u_{i}^{n} .
$$

Observação 3.3.22. O esquema FTFS linear pode ser escrito na forma-I tomando-se $C_{i+1 / 2}=-a R$ e $D_{i+1 / 2}=0$. O esquema de Lax-Wendroff linear, pode ser escrito na forma- $I$ tomando $C_{i+1 / 2}=-a R / 2+a^{2} R^{2} / 2$ e $D_{i+1 / 2}=a R / 2+a^{2} R^{2} / 2$, e o FTFS não linear, pode 
ser escrito na forma-I com $C_{i+1 / 2}=-R a_{i+1 / 2}$ e $D_{i+1 / 2}=0$. Devemos notar que as escolhas para $C_{i+1 / 2}$ e $D_{i+1 / 2}$ não são únicas, mas conforme a proposição acima, serão únicas para escrever o esquema na forma conservativa.

Se escrevermos o esquema de diferenças (3.64) como

$$
u_{i}^{n+1}=u_{i}^{n}-\frac{R}{2} \delta_{0} G_{i}^{n}+\frac{1}{2} \delta_{+}\left(Q_{i-1 / 2}^{n} \delta_{-} u_{i}^{n}\right),
$$

onde $Q$ é chamado de coeficiente de viscosidade numérica, então diremos que o esquema está na forma- $Q$. O esquema (3.110) está na forma conservativa com função de fluxo numérico dada por

$$
g_{i+1 / 2}^{n}=\frac{1}{2}\left(G_{i}^{n}+G_{i+1}^{n}\right)-\frac{1}{2 R} Q_{i+1 / 2}^{n} \delta_{+} u_{i}^{n}
$$

Fazendo

$$
\begin{gathered}
C_{i+1 / 2}^{n}=\frac{1}{2}\left(Q_{i+1 / 2}^{n}-R a_{i+1 / 2}^{n}\right) \\
D_{i+1 / 2}^{n}=\frac{1}{2}\left(Q_{i+1 / 2}^{n}+R a_{i+1 / 2}^{n}\right)
\end{gathered}
$$

transformamos uma equação de diferenças da forma- $Q$ para a forma- $I$. Inversamente, se o esquema de diferenças está na forma conservativa ou forma- $I$, podemos definir o coeficiente de viscosidade numérica $Q$ por

$$
Q_{i+1 / 2}^{n}=R \frac{G\left(u_{i}^{n}\right)+G\left(u_{i+1}^{n}\right)-2 g_{i+1 / 2}^{n}}{\delta_{+} u_{i}^{n}}
$$

ou

$$
Q_{i+1 / 2}^{n}=C_{i+1 / 2}^{n}+D_{i+1 / 2}^{n}
$$

e com isso reescrevemos o esquema na forma-Q.

\subsubsection{Principais Teoremas}

Nesta seçäo iremos apresentar e discutir resultados concernentes a esquemas numéricos com as diferentes propriedades apresentadas anteriormente. Veremos alguns resultados referentes a esquemas-E, esquemas monótonos e TVD. Provaremos alguns desses resultados e apenas citaremos outros. A seguir, enunciaremos e demonstraremos uma proposição que fornece uma condição suficiente para um esquema ser TVD. Este resultado é muito importante pois será a ferramenta principal para provar que um dado esquema é TVD. 
Proposição 3.3.23. Considere um esquema na forma-I como em (3.105). Se

$$
C_{i+1 / 2}^{n} \geq 0, \quad D_{i+1 / 2}^{n} \geq 0 \quad \text { e } \quad C_{i+1 / 2}^{n}+D_{i+1 / 2}^{n} \leq 1,
$$

então o esquema é TVD.

Demonstração: Começamos aplicando $\delta_{+}$em ambos os lados da equação (3.105), a seguir tomamos o valor absoluto e somamos de $-\infty$ a $\infty$, obtendo

$T V\left(\mathbf{u}^{n+1}\right)=\sum_{i=-\infty}^{\infty}\left|\delta_{+} u_{i}^{n+1}\right|=\sum_{i=-\infty}^{\infty}\left|\delta_{+} u_{i}^{n}+\delta_{+}\left(C_{i+1 / 2}^{n} \delta_{+} u_{i}^{n}\right)-\delta_{+}\left(D_{i-1 / 2}^{n} \delta_{-} u_{i}^{n}\right)\right|$

$\left.=\sum_{i=-\infty}^{\infty} \mid \delta_{+} u_{i}^{n}+C_{i+3 / 2}^{n} \delta_{+} u_{i+1}^{n}-C_{i+1 / 2}^{n} \delta_{+} u_{i}^{n}-D_{i+1 / 2}^{n} \delta_{+} u_{i+1}^{n}\right)+D_{i-1 / 2}^{n} \delta_{-} u_{i}^{n} \mid$

$=\sum_{i=-\infty}^{\infty}\left|\delta_{+} u_{i}^{n}+C_{i+3 / 2}^{n} \delta_{+} u_{i+1}^{n}-C_{i+1 / 2}^{n} \delta_{+} u_{i}^{n}-D_{i+1 / 2}^{n} \delta_{+} u_{i}^{n}+D_{i-1 / 2}^{n} \delta_{+} u_{i-1}^{n}\right| \quad\left(\right.$ pois $\delta_{-} u_{i+1}^{n}=$ $\delta_{+} u_{i}^{n}$ e $\left.\delta_{-} u_{i}^{n}=\delta_{+} u_{i-1}^{n}\right)$

$\leq \sum_{i=-\infty}^{\infty} C_{i+3 / 2}^{n}\left|\delta_{+} u_{i+1}^{n}\right|+\sum_{i=-\infty}^{\infty}\left(1-C_{i+1 / 2}^{n}-D_{i+1 / 2}^{n}\right)\left|\delta_{+} u_{i+1}^{n}\right|+\sum_{i=-\infty}^{\infty} D_{i-1 / 2}^{n}\left|\delta_{+} u_{i-1}^{n}\right| \quad$ (reagrupando e usando a hipótese)

$=\sum_{i=-\infty}^{\infty} C_{j+1 / 2}^{n}\left|\delta_{+} u_{j}^{n}\right|+\sum_{i=-\infty}^{\infty}\left(1-C_{i+1 / 2}^{n}-D_{i+1 / 2}^{n}\right)\left|\delta_{+} u_{i}^{n}\right|+\sum_{i=-\infty}^{\infty} D_{j+1 / 2}^{n}\left|\delta_{+} u_{j}^{n}\right| \quad$ (fazendo uma mudança de índice $i=j+1$ )

$=\sum_{i=-\infty}^{\infty}\left|\delta_{+} u_{i}^{n}\right|=T V\left(\mathbf{u}^{n}\right)$.

Proposição 3.3.24. Um esquema-E conservativo que satisfaz

$$
R\left|\left(g_{i+1 / 2}^{n}-G_{i}^{n}\right)+\left(g_{i+1 / 2}^{n}-G_{i+1}^{n}\right)\right| \leq\left|\delta_{+} u_{i}^{n}\right|
$$

é TVD.

Demonstração: Considere um esquema-E na forma conservativa (3.65). S€ escrevermos o esquema na forma-I, então

$$
C_{i+1 / 2}^{n}=-R \frac{g_{i+1 / 2}^{n}-G\left(u_{i}^{n}\right)}{\delta_{+} u_{i}^{n}}
$$

e

$$
D_{i+1 / 2}^{n}=-R \frac{g_{i+1 / 2}^{n}-G\left(u_{i+1}^{n}\right)}{\delta_{+} u_{i}^{n}} .
$$

Se $u_{i}^{n} \leq u_{i+1}^{n}$, e considerando um esquema-E, temos que $g_{i+1 / 2}^{n} \leq G\left(u_{i}^{n}\right)$ e portanto $C_{i+1 / 2}^{n} \geq$ 0 . Igualmente, $g_{i+1 / 2}^{n} \leq G\left(u_{i+1}^{n}\right)$ e assim $D_{i+1 / 2}^{n} \geq 0$. Similarmente, se $u_{i}^{n}>u_{i+1}^{n}$, então 
$C_{i+1 / 2}^{n} \geq 0$ e $D_{i+1 / 2}^{n} \geq 0$. Como

$$
\begin{aligned}
C_{i+1 / 2}^{n}+D_{i+1 / 2}^{n} & =-R \frac{g_{i+1 / 2}^{n}-G\left(u_{i}^{n}\right)}{\delta_{+} u_{i}^{n}}-R \frac{g_{i+1 / 2}^{n}-G\left(u_{i+1}^{n}\right)}{\delta_{+} u_{i}^{n}} \\
& =R\left|\frac{g_{i+1 / 2}^{n}-G\left(u_{i}^{n}\right)}{\delta_{+} u_{i}^{n}}+\frac{g_{i+1 / 2}^{n}-G\left(u_{i+1}^{n}\right)}{\delta_{+} u_{i}^{n}}\right| \\
& \leq 1,
\end{aligned}
$$

temos pela Proposição 3.3.23 temos que o esquema é TVD.

Proposição 3.3.25. Esquemas-E são no máximo de primeira ordem de precisão.

Demonstração: Para esquemas-E, temos através da definição 3.3.11, que

$$
\left\{g\left(u_{i-p}, \ldots, u_{i}, u_{i+1}, \ldots, u_{i+q}\right)-g(u, \ldots, u)\right\} / \delta_{+} u_{i} \leq 0
$$

para todo $u$ entre $u_{i+1}$ e $u_{i}$. Seja

$$
u_{i+j}=u_{i}+\alpha_{j}\left(u_{i+1}-u_{i}\right), \text { com } \alpha_{0}=0, \alpha_{1}=1
$$

Vamos adotar a notação simplificada para as derivadas:

$$
g_{j}=g_{j}\left(u_{i-p}, \ldots, u_{i+q}\right)=\frac{\partial g}{\partial u_{i+j}}\left(u_{i-p}, \ldots, u_{i+q}\right) .
$$

Caso 1: $\alpha_{1}=1, \alpha_{j}=0, j \neq 1 ; u=u_{i}$. Pela hipótese,

$$
\left\{g\left(u_{i}, \ldots, u_{i}, u_{i+1}, u_{i}, \ldots, u_{i}\right)-g\left(u_{i}, \ldots, u_{i}\right)\right\} /\left(u_{i+1}-u_{i}\right) \leq 0
$$

Quando $u_{i+1}$ tende a $u_{i}$; temos

$$
g_{1}(u, \ldots, u) \leq 0
$$

Caso 2: $\alpha_{0}=0, \alpha_{j}=1, j \neq 0 ; u=u_{i+1}$.

$$
\left\{g\left(u_{i+1}, \ldots, u_{i+1}, u_{i}, u_{i+1}, \ldots, u_{i+1}\right)-g\left(u_{i+1}, \ldots, u_{i+1}\right)\right\} /\left(u_{i+1}-u_{i}\right) \leq 0
$$

Fazendo agora $u_{i}$ tender a $u_{i+1}$; temos

$$
g_{0}(u, \ldots, u) \geq 0
$$

Caso 3: $\alpha_{0}=0, \alpha_{1}=1, \alpha_{j}=0, j \neq j_{0}$ para algum $j_{0} \neq 0,1 ; u=u_{i}$.

$$
\left\{g\left(u_{i}, \ldots, u_{i}, u_{i+1}, u_{i}, \ldots, u_{i}+\alpha_{j_{0}}\left(u_{i+1}-u_{i}\right), \ldots, u_{i}\right)-g\left(u_{i}, \ldots, u_{i}\right)\right\} /\left(u_{i+1}-u_{i}\right) \leq 0
$$


Quando $u_{i+1}$ tende a $u_{i}$; temos

$$
g_{1}(u, \ldots, u)+\alpha_{j_{0}} g_{j_{0}}(u, \ldots, u) \leq 0
$$

para todo $\alpha_{j_{0}}$. Isto implica que $g_{j_{0}}(u, \ldots, u)=0$, para $j_{0} \neq 0,1$. Assim $W\left(u_{i-p-1}, \ldots, u_{i+q}\right)=$ $u_{i}-R\left\{g\left(u_{i-p}, \ldots, u_{i+q}\right)-g\left(u_{i-p-1}, \ldots, u_{i+q-1}\right)\right\}$ e para cada inteiro $j$ entre $-p-1$ e $q$, temos

$$
\begin{aligned}
W_{j}(u, \ldots, u) & =0 \text { para } \quad|j|>1, \\
W_{1}(u, \ldots, u) & =-R g_{1}(u, \ldots, u) \geq 0, \\
W_{-1}(u, \ldots, u) & =+R g_{0}(u, \ldots, u) \geq 0,
\end{aligned}
$$

ou seja,

$$
\frac{\partial W}{\partial u_{i+j}}(u, \ldots, u) \geq 0 .
$$

Utilizando a Observação 3.3.15 e a Proposição 3.3 .30 enunciada mais a frente, a qual diz que esquemas monótonos sào de prim̄eira ordem de precisão, concluímos que um esquema-E é de primeira ordem de precisão.

A seguir, avançamos no estudo de esquemas monótonos. A consideração de tais esquemas nâo se deve ao fato dos esquemas-E serem de primeira ordem mas sim do fato de que é mais fácil provar resultados para esquemas monótonos do que resultados análogos para esquemas-E. Entretanto, como veremos do resultado seguinte, muitos esquemas monótonos são esquemas- $E$ e portanto autornaticamente de primeira ordem de precisão.

Proposiçāo 3.3.26. Esquemas monótonos conservativos de três pontos são esquemas-E.

Demonstração: Se escrevermos esse esquema na forma conservativa como

$$
\begin{aligned}
u_{i}^{n} & =u_{i}^{n}-R\left[g_{i+1 / 2}^{n}-g_{i-1 / 2}^{n}\right] \\
& =u_{i}^{n}-R\left[g\left(u_{i}^{n}, u_{i+1}^{n}\right)-g\left(u_{i-1}^{n}, u_{i}^{n}\right)\right]
\end{aligned}
$$

podemos usar a monotonicidade para mostrar que $g$ é crescente com respeito ao primeiro argumento e decrescente em relação ao segundo. Sabemos que a consistência implica em $g(u, u)=G(u)$. Suponha agora que $u_{i}<u_{i+1}$ e $u \in\left[u_{i}, u_{i+1}\right]$. Assim,

$$
\begin{aligned}
g_{i+1 / 2} & =g\left(u_{i}, u_{i+1}\right) \\
& \leq g\left(u, u_{i+1}\right) \quad(g \text { é crescente com respeito a seu primeiro argumento }) \\
& \leq g(u, u)=G(u) \quad(g \text { é decrescente com respeito a seu segundo argumento }) .
\end{aligned}
$$

Similarmente verifica-se para o caso $u_{i+1}<u_{i}$. Portanto, o esquema é um esquema-E. 
Observação 3.3.27. Na demonstraçâo dessa proposição utilizamos um resultado apresentado em [15] pag. 124, o qual afirma que a função de fluxo numérico $g(u, v)$ de um esquema monótono, conservativo de três pontos é crescente em relação a seu primeiro argumento $e$ decrescente em relação ao segundo argumento.

Proposição 3.3.28. Esquemas monótonos conservativos são TVD.

Demonstração: Notamos que

$$
\begin{aligned}
T V\left(\mathrm{u}^{n+1}\right) & =\sum_{i=-\infty}^{\infty}\left|\delta_{+} u_{i}^{n+1}\right|=\sum_{i=-\infty}^{\infty}\left|u_{i+1}^{n+1}-u_{i}^{n+1}\right| \\
& =\sum_{i=-\infty}^{\infty}\left|W\left(u_{i+1-(p+1)}^{n}, \ldots, u_{i+1+q}^{n}\right)-W\left(u_{i-(p+1)}^{n}, \ldots, u_{i+q}^{n}\right)\right| \\
& =\sum_{i=-\infty}^{\infty}\left|W\left(u_{i-(p+1)}^{n}+\delta_{+} u_{i-(p+1)}^{n}, \ldots, u_{i+q}^{n}+\delta_{+} u_{i+q}^{n}\right)-W\left(u_{i-(p+1)}^{n}, \ldots, u_{i+q}^{n}\right)\right| .
\end{aligned}
$$

Temos então

$$
\begin{aligned}
& \frac{d}{d t} W\left(u_{i-(p+1)}^{n}+t \delta_{+} u_{i-(p+1)}^{n}, \ldots, u_{i+q}^{n}+t \delta_{+} u_{i+q}^{n}\right) \\
& =\sum_{j=-(p+1)}^{q} W_{j}\left(u_{i-(p+1)}^{n}+t \delta_{+} u_{i-(p+1)}^{n}, \ldots, u_{i+q}^{n}+t \delta_{+} u_{i+q}^{n}\right) \delta_{+} u_{i+j}^{n}, \\
& \text { e portanto, } \\
& \sum_{j=-(p+1)}^{q} \int_{0}^{1} W_{j}\left(u_{i-(p+1)}^{n}+t \delta_{+} u_{i-(p+1)}^{n}, \ldots, u_{i+q}^{n}+t \delta_{+} u_{i+q}^{n}\right) \delta_{+} u_{i+j}^{n} d t \\
& =W\left(u_{i-(p+1)}^{n}+\delta_{+} u_{i-(p+1)}^{n}, \ldots, u_{i+q}^{n}+\delta_{+} u_{i+q}^{n}\right)-W\left(u_{i-(p+1)}^{n}, \ldots, u_{i+q}^{n}\right) .
\end{aligned}
$$

$W_{j}$ representa a derivada parcial de $W$ com respeito às $(p+2+j)$-ésima variáveis. Para $j=-(p+1)$ corresponde a diferenciação em relação à primeira variável, $j=-p$ em relação à segunda variável, $\ldots, j=q$ corresponde a diferenciação em relação à $(p+q+2)$ variável. Continuando o cálculo acima temos

$$
T V\left(\mathrm{u}^{n+1}\right)=\sum_{j=-\infty}^{\infty}\left|\sum_{j=-(p+1)}^{q} \int_{0}^{1} W_{j}\left(u_{i-(p+1)}^{n}+t \delta_{+} u_{i-(p+1)}^{n}, \ldots, u_{i+q}^{n}+t \delta_{+} u_{i+q}^{n}\right) \delta_{+} u_{i+j}^{n} d t\right| .
$$

Então, usando a desigualdade triangular, tomando o valor absoluto no interior da integral, usando o fato de que o esquema de diferenças é monótono (as derivadas parciais são não negativas) e fazendo uma mudança de índice $m=i+j$, temos

$$
T V\left(\mathrm{u}^{n+1}\right) \leq \sum_{i=-\infty}^{\infty} \sum_{,=-(p+1)}^{q} \int_{0}^{1} W_{j}\left(u_{i-(p+1)}^{n}+t \delta_{+} u_{i-(p+1)}^{n}, \ldots, u_{i+q}^{n}+t \delta_{+} u_{i+q}^{n}\right)\left|\delta_{+} u_{i+j}^{n}\right| d t
$$




$$
=\sum_{m=-\infty}^{\infty} \sum_{j=-(p+1)}^{q} \int_{0}^{1} W_{j}\left(u_{m-j-(p+1)}^{n}+t \delta_{+} u_{m-j-(p+1)}^{n}, \ldots, u_{m-j+q}^{n}+t \delta_{+} u_{m-j+q}^{n}\right)\left|\delta_{+} u_{m}^{n}\right| d t .
$$

Pelo fato do esquema scr conservativo, sabemos que podemos escrevê-lo como

$$
W\left(w_{l-(p+1)}, \ldots, w_{l+q}\right)=w_{l}-R\left[g\left(w_{l-p}, \ldots, w_{l+q}\right)-g\left(w_{l-p-1}, \ldots, w_{l+q-1}\right)\right] .
$$

A diferenciação de (3.119) em relação a seus argumentos, fornece

$$
\begin{aligned}
W_{-(p+1)} & =R g_{1}\left(w_{l-p-1}, \ldots, w_{l+q-1}\right) \\
W_{j} & =\delta_{j l}-R\left[g_{j+p+1}\left(w_{l-p}, \ldots, w_{l+q}\right)-g_{j+p+2}\left(w_{l-p-1}, \ldots, w_{l+q-1}\right)\right] \\
W_{q} & =-R g_{q+p+1}\left(w_{l-p}, \ldots, w_{l+q}\right)
\end{aligned}
$$

onde $j=-p, \ldots, q-1$ para a equação (3.121). A notaçào utilizada acima (e durante o resto dessa demonstração) é diferente do que usamos no passado. Ela não emprega o superescrito e o índice subescrito não tem o mesmo significado anteriormente usado. Aqui os $g_{j}^{\prime} s$ denotam a diferenciação parcial de $g$ com respeito a $j$-ésima variável. A mudança foi uma tentativa de tornar a demonstração um pouco mais compreenssível. O termo $\delta_{i j}$ representa o delta de Kronecker. Somando (3.120)-(3.121) obtemos

$$
\begin{aligned}
& \sum_{j=-(p+1)}^{q} W_{j}\left(u_{m-j-(p+1)}^{n}, \ldots, u_{m-j+q}^{n}\right) \\
= & R g_{1}\left(w_{m}, \ldots, w_{m+(p+1)+q}\right)+1-R \sum_{j=-p}^{q-1}\left[g_{j+p+1}\left(w_{m-j-p} \ldots, w_{m-j+q}\right)\right. \\
& \left.-g_{j+p+2}\left(w_{m-j-p-1}, \ldots, w_{m-j+q-1}\right)\right]-R g_{q+p}\left(w_{m-q-p}, \ldots, w_{m}\right) \\
= & R g_{1}\left(w_{m}, \ldots, w_{m+(p+1)+q}\right) 1-R \sum_{j=-p}^{q-1} g_{j+p+1}\left(w_{m-j-p}, \ldots, w_{m-j+q}\right) \\
& +R \sum_{j=-p}^{q-1} g_{j+p+2}\left(w_{m-j-p-1}, \ldots, w_{m-j+q-1}\right)-R g_{q+p}\left(w_{m-q-p}, \ldots, w_{m}\right) \\
= & R g_{1}\left(w_{m}, \ldots, w_{m+(p+1)+q}\right) 1-R \sum_{j=-p}^{q-1} g_{j+p+1}\left(w_{m-j-p}, \ldots, w_{m-j+q}\right) \\
& +R \sum_{j=-p+1}^{q} g_{l+p-1}\left(w_{m-l-p}, \ldots, w_{m-l+q}\right)-R g_{q+p}\left(w_{m-q-p}, \ldots, w_{m}\right) \\
= & R g_{1}\left(w_{m}, \ldots, w_{m+(p+1)+q}\right)+1-R g_{1}\left(w_{m}, \ldots, w_{m+p+q}\right) \\
& +R g_{q+p}\left(w_{m-q-p}, \ldots, w_{m}\right)-R g_{q+p}\left(w_{m-q-p}, \ldots, w_{m}\right)=1 .
\end{aligned}
$$

Aplicando esta identidade para a expressão (3.118) obtemos

$$
T V\left(\mathbf{u}^{n+1}\right) \leq \sum_{m=-\infty}^{\infty} \int_{0}^{1}\left|\delta_{+} u_{m}^{n}\right| d t=\sum_{m=-\infty}^{\infty}\left|\delta_{+} u_{m}^{n}\right|=T V\left(\mathbf{u}^{n}\right)
$$

que é o que queríamos provar. 
A seguir incluimos um resultado que está relacionado com o erro de truncamento de um esquema de diferenças que será de ajuda para provar a proposição 3.3 .30 dada abaixo. Gostaríamos de notar que este resultado é um resultado geral que pode ser usado em uma variedade de maneiras e vai ser usado mais a frente.

Proposiçāo 3.3.29. O erro de truncamento de um esquema de diferenças da forma

$$
u_{i}^{n+1}=W\left(u_{i-(p+1)}^{n}, \ldots, u_{i+q}^{n}\right)
$$

é dado por

$$
\Delta t \tau_{i}^{n}=-\Delta t^{2}\left\{\left[q(u) u_{x}\right]_{x}\right\}_{u=v_{i}^{n}}+o\left(\Delta t\left(\Delta t^{2}+\Delta x^{3}\right)\right)
$$

onde $v=v(x, t)$ é uma solução da lei de conservação (3.98) e

$$
q(u)=\frac{1}{2}\left[\frac{1}{R^{2}} \sum_{j=-(p+1)}^{q} j^{2} W_{j}(u, \ldots, u)-\left[G^{\prime}(u)\right]^{2}\right] .
$$

Demonstração: Começamos expandindo $\Delta t \tau_{i}^{n}=v_{i}^{n+1}-W\left(v_{i-(p+1)}^{n}, \ldots, v_{i+q}^{n}\right)$ em série de Taylor, onde $v=v(x, t)$ é uma solução da lei de conservação (3.98), como

$$
\begin{aligned}
\Delta t \tau_{i}^{n}= & v_{i}^{n+1}-W\left(v_{i-(p+1)}^{n}, \ldots, v_{i+q}^{n}\right) \\
= & v_{i}^{n}+\Delta t\left(v_{t}\right)_{i}^{n}+\frac{\Delta t^{2}}{2}\left(v_{t t}\right)_{i}^{n}-W\left(v_{i}^{n}, \ldots, v_{i}^{n}\right)-\sum_{j=-(p+1)}^{q} W_{j}\left(v_{i}^{n}, \ldots, v_{i}^{n}\right)\left(v_{i+j}^{n}-v_{i}^{n}\right) \\
& -\frac{1}{2} \sum_{j=-(p+1)}^{q} \sum_{m=-(p+1)}^{q} W_{k m}\left(v_{i}^{n}, \ldots, v_{i}^{n}\right)\left(v_{i+j}^{n}-v_{i}^{n}\right)\left(v_{i+m}^{n}-v_{i}^{n}\right) \\
& +o\left(\Delta t^{3}\right)+o\left(\Delta t \Delta x^{3}\right) .
\end{aligned}
$$

Expandindo $v_{i+j}^{n}$ e $v_{i+m}^{n}$ ao redor de $v_{i}^{n}$ fornece

$$
\begin{aligned}
\Delta t \tau_{i}^{n}= & v_{i}^{n}+\Delta t\left(v_{t}\right)_{i}^{n}+\frac{\Delta t^{2}}{2}\left(v_{t t}\right)_{i}^{n}-W\left(v_{i}^{n}, \ldots, v_{i}^{n}\right) \\
= & -\Delta x\left(v_{x}\right)_{i}^{n} \sum_{j=-(p+1)}^{q} j W_{j}\left(v_{i}^{n}, \ldots, v_{i}^{n}\right)\left(v_{i+j}^{n}-v_{i}^{n}\right) \\
& -\frac{\Delta x^{2}}{2}\left(v_{x x}\right)_{i}^{n} \sum_{j=-(p+1)}^{q} j^{2} W_{j}\left(v_{i}^{n}, \ldots, v_{i}^{n}\right) \\
& -\frac{\Delta x^{2}}{2}\left[\left(v_{x}\right)_{i}^{n}\right]^{2} \sum_{j=-(p+1)}^{q} \sum_{m=-(p+1)}^{q} j m W_{j m}\left(v_{i}^{n}, \ldots, v_{i}^{n}\right)+o\left(\Delta t\left(\Delta t^{2}+\Delta x^{3}\right)\right) .
\end{aligned}
$$


Como estamos trabalhando com esquemas conservativos, $W$ pode ser escrito como em (3.119), e vemos que

$$
\left[W\left(v_{i}^{n}, \ldots, v_{i}^{n}\right)=v_{i}^{n}-R\left[g\left(v_{i}^{n}, \ldots, v_{i}^{n}\right)-g\left(v_{i}^{n}, \ldots, v_{i}^{n}\right)\right]=v_{i}^{n} .\right.
$$

Também, usando a forma conservativa do esquema, temos que

$$
\sum_{j=-(p+1)}^{q} \sum_{m=-(p+1)}^{q} j m W_{j m}\left(v_{i}^{n}, \ldots, v_{i}^{n}\right)=\sum_{j=-(p+1)}^{q} \sum_{m=-(p+1)}^{q} j^{2} W_{j m}\left(v_{i}^{n}, \ldots, v_{i}^{n}\right)
$$

O erro de truncamento pode então ser escrito como

$$
\begin{aligned}
\Delta t \tau_{i}^{n}= & \Delta t\left(v_{t}\right)_{i}^{n}-\Delta x\left(v_{x}\right)_{i}^{n} \sum_{j=-(p+1)}^{q} j W_{j}\left(v_{i}^{n}, \ldots, v_{i}^{n}\right)+\frac{\Delta t^{2}}{2}\left(v_{t t}\right)_{i}^{n} \\
& -\frac{\Delta x^{2}}{2}\left[\left(v_{x x}\right)_{i}^{n} \sum_{j=-(p+1)}^{q} j^{2} W_{j}\left(v_{i}^{n}, \ldots, v_{i}^{n}\right)\right. \\
& \left.+\left[\left(v_{x}\right)_{i}^{n}\right]^{2} \sum_{j=-(p+1)}^{q} \sum_{m=-(p+1)}^{q} j^{2} W_{j m}\left(v_{i}^{n}, \ldots, v_{i}^{n}\right)\right]+o\left(\Delta t\left(\Delta t^{2}+\Delta x^{3}\right)\right) .
\end{aligned}
$$

Usando o fato de que

$$
\sum_{j=-(p+1)}^{q} j W_{j}\left(v_{i}^{n}, \ldots, v_{i}^{n}\right)=-R\left[G^{\prime \prime}(v)\right]_{i}^{n}
$$

que é necessário para consistência, e o fato de que

$$
j^{2} \sum_{m=-(p+1)}^{q} W_{j m}\left(v_{i}^{n}, \ldots, v_{i}^{n}\right)\left(v_{x}\right)_{i}^{n}=\left[\left(j^{2} W_{j}(v, \ldots, v)\right)_{x}\right]_{i}^{n}
$$

podemos eliminar os primeiros dois termos da equação (3.127) (devido $v_{t}+G^{\prime}(v) v_{x}=0$ ) e escrevemos o erro de truncamento como

$$
\begin{aligned}
\Delta t \tau_{i}^{n}= & \frac{\Delta t^{2}}{2}\left(v_{t t}\right)_{i}^{n}-\frac{\Delta x^{2}}{2}\left(v_{x x}\right)_{i}^{n} \sum_{j=-(p+1)}^{q} j^{2} W_{j}\left(v_{i}^{n}, \ldots, v_{i}^{n}\right)+ \\
& -\frac{\Delta x^{2}}{2}\left(v_{x}\right)_{i}^{n} \sum_{j=-(p+1)}^{q}\left[\left(j^{2} W_{j}\left(v_{i}^{n}, \ldots, v_{i}^{n}\right)\right)_{x}\right]_{i}^{n}+o\left(\Delta t\left(\Delta t^{2}+\Delta x^{3}\right)\right)
\end{aligned}
$$

Se usarmos o fato de que $v$ é uma solução da lei de conservação (3.98), podemos derivar a seguinte identidade

Eliminando $v_{t t}$ de $(3.130)$ temos

$$
v_{t t}=\left[\left(G^{\prime}(v)\right)^{2} v_{x}\right]_{x}
$$

$$
\Delta t \tau_{i}^{n}=\frac{\Delta t^{2}}{2}\left\{\left[\left(\left(G^{\prime}(v)\right)^{2}-\frac{1}{R^{2}} \sum_{j=-(p+1)}^{q} j^{2} W_{j}(v, \ldots, v)\right) v_{x}\right]_{x}\right\}_{i}^{n}+o\left(\Delta t\left(\Delta t^{2}+\Delta x^{3}\right)\right)
$$

que é o que tínhamos para provar. 
Proposição 3.3.30. Esquemas monótonos são quase sempre de primeira ordem de precisão.

Demonstração: Começamos notando que

$$
-R G^{\prime}\left(v_{i}^{n}\right)=\sum_{j=-(p+1)}^{q} j W_{j}\left(v_{i}^{n}, \ldots, v_{i}^{n}\right) .
$$

Então

$$
\begin{aligned}
R^{2}\left[G^{\prime}\left(v_{i}^{n}\right)\right]^{2} & =\left(\sum_{j=-(p+1)}^{q} j W_{j}\left(v_{i}^{n}, \ldots, v_{i}^{n}\right)\right)^{2} \\
& =\left(\sum_{j=-(p+1)}^{q} j \sqrt{W_{j}\left(v_{i}^{n}, \ldots, v_{i}^{n}\right)} \sqrt{W_{j}\left(v_{i}^{n}, \ldots, v_{i}^{n}\right)}\right)^{2} \\
& \leq \sum_{j=-(p+1)}^{q} j^{2} W_{j}\left(v_{i}^{n}, \ldots, v_{i}^{n}\right) \sum_{j=-(p+1)}^{q} W_{j}\left(v_{i}^{n}, \ldots, v_{i}^{n}\right) \\
& =\sum_{j=-(p+1)}^{q} j^{2} W_{j}\left(v_{i}^{n}, \ldots, v_{i}^{n}\right) .
\end{aligned}
$$

Esta desigualdade implica que $q\left(v_{i}^{n}\right) \geq 0$. Pela desigualdade de Schwarz, a igualdade na equação (3.133) ocorre quando $j W_{j}\left(v_{i}^{n}, \ldots, v_{i}^{n}\right)=c W_{j}\left(v_{i}^{n}, \ldots, v_{i}^{n}\right)$ para alguma constante $c$. Portanto,

$$
W_{j}\left(v_{i}^{n}, \ldots, v_{i}^{n}\right)=0, \forall j,
$$

e assim temos que $W$ é uma função constante. Neste caso, o esquema de diferenças será uma translação pura. Assim a menos do caso em que $W$ é constante a proposição é válida, e este é o caso a que nos referimos como "quase sempre" no enunciado.

A seguir enunciamos e demonstramos alguns resultados importantes. Esses resultados são úteis pois eles nos mostrarão que para obter um esquema de alta ordem, não devemos considerar esquemas lineares nem esquemas de três de pontos.

Proposição 3.3.31. Um esquema de diferenças linear TVD é no máximo de primeira ordem.

Demonstração: Consideramos um esquema de diferenças da forma

$$
u_{i}^{n+1}=\sum_{j=-q}^{p} a_{j} u_{i+j}^{n}
$$

e a função

$$
u_{i+j}^{n}= \begin{cases}1 & \text { se } j \leq 0 \\ 0 & \text { se } j>0\end{cases}
$$


Então $T V\left(\mathbf{u}^{n}\right)=1$, e

$$
T V\left(\mathbf{u}^{n+1}\right)=\sum_{i=-\infty}^{\infty}\left|\delta_{+} u_{i}^{n+1}\right|=\sum_{i=-\infty}^{\infty}\left|\sum_{j=-q}^{p} a_{j} \delta_{+} u_{i+j}^{n}\right|=\sum_{j=-q}^{p}\left|a_{j}\right|
$$

O esquema de diferenças (3.134) é consistente se

$$
\sum_{j=-q}^{p} a_{j}=1
$$

Portanto, se $a_{j}<0$ para algum. $j$,

$$
T V\left(\mathbf{u}^{n+1}\right)=\sum_{j=-q}^{p}\left|a_{j}\right|>1=T V\left(\mathbf{u}^{n}\right)
$$

e o esquema não é TVD. Assim devemos ter $a_{j} \geq 0$ para todo $j,-q \leq j \leq p$. Desta forma o esquema é monótono, e pela proposiçāo (3.3.30) o esquema é no máximo de primeira ordem de precisão.

Acabamos de demonstrar que não podemos ter esquemas lineares de segunda ordem TVD. O objetivo agora será construir esquemas não-lineares TVD que possuam ordem maior que um. Uma das maneiras para se obter esquemas de segunda ordem de precisão será modificando o esquema de Lax-Wendroff. Sejam $g_{i+1 / 2}^{\mathrm{Lw}}$ a função de fluxo numérico associada com o esquema de Lax-Wendroff (3.93) e $g_{i+1 / 2}^{n}$ a função para o esquema que estamos considerando. A proposição abaixo garante que se a diferença entre as funções de fluxo numérico desses dois métodos for de segunda ordem de precisào, o método que estamos considerando também será de segunda ordem.

Proposição 3.3.32. Se

$$
g_{i+1 / 2}^{n}-g_{i+1 / 2}^{L W}=o\left(\Delta x^{2}\right)
$$

e o erro principal associado com o termo de o $\left(\Delta x^{2}\right)$ é suave, entäo o esquema associado com a função de fluxo numérico $g_{i+1 / 2}^{n}$ é de segunda ordem de precisão.

Demonstração: Como o esquema de Lax-Wendroff é de segunda ordem de precisão, temos

$$
\frac{u^{n+1}-u_{i}^{n}}{\Delta t}+\frac{\delta_{-} g_{i+1 / 2}^{\mathrm{LW}}}{\Delta x}-\left[u_{t}+G(u)_{x}\right]_{i}^{n}=o\left(\Delta x^{2}\right)
$$

Se somarmos o termo $\left(\frac{\delta_{-} g_{i+1 / 2}^{n}}{\Delta x}-\frac{\delta_{-} g_{i+1 / 2}^{\mathrm{LW}}}{\Delta x}\right)$ em ambos os lados de (3.136). temos que 


$$
\frac{u^{n+1}-u_{i}^{n}}{\Delta t}+\frac{\delta_{-} g_{i+1 / 2}^{n}}{\Delta x}-\left[u_{t}+G(u)_{x}\right]_{i}^{n}=o\left(\Delta x^{2}\right)+\frac{\left(g_{i+1 / 2}^{n}-g_{i+1 / 2}^{\mathrm{Lw}}\right)-\left(g_{i-1 / 2}^{n}-g_{i-1 / 2}^{\mathrm{LW}}\right)}{\Delta x} .
$$

Pela hipótese, $g_{i+1 / 2}^{n}-g_{i+1 / 2}^{\mathrm{LW}}=o\left(\Delta x^{2}\right)$ e o termo principal de $o\left(\Delta x^{2}\right)$ é suave, $\delta_{-}\left(g_{i+1 / 2}^{n}-\right.$ $\left.g_{i+1 / 2}^{\mathrm{LW}}\right)=o\left(\Delta x^{3}\right)$. Dividindo essa igualdade por $\Delta x$ reduzimos a ordem do erro, ou seja,

$$
\frac{\left(g_{i+1 / 2}^{n}-g_{i+1 / 2}^{\mathrm{LW}}\right)-\left(g_{i-1 / 2}^{n}-g_{i-1 / 2}^{\mathrm{LW}}\right)}{\Delta x}=o\left(\Delta x^{2}\right) .
$$

Usando este resultado em (3.137), temos que

$$
\frac{u^{n+1}-u_{i}^{n}}{\Delta t}+\frac{\delta_{-} g_{i+1 / 2}^{n}}{\Delta x}-\left[u_{t}+G(u)_{x}\right]_{i}^{n}=o\left(\Delta x^{2}\right),
$$

que é o que queríamos provar.

O método mais comum para provar que algum esquema é TVD (ou forçar) é usar a proposição 3.3.23. Infelizmente essa proposição não fornece a condição necessária para um esquema ser TVD. Para obtermos esquemas de alta ordem, definimos a seguinte subclassc de esquemas TVD.

Definição 3.3.33. Um esquema de diferenças é dito ser TVD incremental se $C_{i+1 / 2}^{n} \geq$ $0, D_{i+1 / 2}^{n} \geq 0$ e $C_{i+1 / 2}^{n}+D_{i+1 / 2}^{n} \leq 1$.

Observação 3.3.34. A razão dessa definição é determinar uma classe de esquemas TVD para podermos provar as Proposições (3.3.36, 3.3.38 e 3.3.39). Podem existir esquemas TVD que não satisfaçam as condições $C_{i+1 / 2}^{n} \geq 0, D_{i+1 / 2}^{n} \geq 0$ e $C_{i+1 / 2}^{n}+D_{i+1 / 2}^{n} \leq 1$. A definição 3.3.33 e a proposição 3.3 .23 fornecem uma condição necessária e suficiente para um esquema ser incrementalmente TVD. A referência /44J diz que pode ser que a Proposição (3.3.23) seja uma condição necessária e suficiente para um esquema ser TVD, mas a volta é difícil de demonstrar. Assim sendo, apresentamos a versão "se e somente se"da Proposição 3.3.23.

Proposiçāo 3.3.35. Considere um esquema de diferenças na forma-I como em (3.105). Então o esquema de diferenças (3.105) é incrementalmente TVD se e somente se

$$
C_{i+1 / 2}^{n} \geq 0, \quad D_{i+1 / 2}^{n} \geq 0 \quad \text { e } \quad C_{i+1 / 2}^{n}+D_{i+1 / 2}^{n} \leq 1
$$

Provaremos agora os seguintes três resultados. 
Proposição 3.3.36. Um esquema conservativo é TVD incremental se e só se o coeficiente de viscosidade numérica satisfaz

$$
R\left|a_{i+1 / 2}^{n}\right| \leq Q_{i+1 / 2}^{n} \leq 1
$$

para todo $i$ onde $a_{i+1 / 2}^{n}$ é a velocidade local da onda definida em (3.55).

Demonstração: Usando as expressões (3.112), (3.113), vemos que um esquema na forma-Q é relacionado com um na forma-I via

$$
\begin{aligned}
& C_{i+1 / 2}^{n}=\frac{1}{2}\left(Q_{i+1 / 2}^{n}-R a_{i+1 / 2}^{n}\right) \\
& D_{i+1 / 2}^{n}=\frac{1}{2}\left(Q_{i+1 / 2}^{n}+R a_{i+1 / 2}^{n}\right) .
\end{aligned}
$$

Iremos aplicar a proposição 3.3.35 e assumir que o esquema é TVD incremental. As condições $C_{i+1 / 2}^{n} \geq 0, D_{i+1 / 2}^{n} \geq 0$ implicam que $Q_{i+1 / 2}^{n} \geq R a_{i+1 / 2}^{n}$ e $Q_{i+1 / 2}^{n} \geq-R a_{i+1 / 2}^{n}$, respectivamente. $\mathrm{Na}$ verdade, essas duas expressōes resultam em $Q_{i+1 / 2}^{n} \geq R\left|a_{i+1 / 2}^{n}\right|$. Somando (3.140) e (3.141), temos da Proposição 3.3 .35 que $C_{i+1 / 2}^{n}+D_{i+1 / 2}^{n} \leq 1$. Portanto, $R\left|a_{i+1 / 2}^{n}\right| \leq Q_{i+1 / 2}^{n} \leq$ 1. Inversamente, se multiplicarmos (3.139) por 1/2 e usarmos a propriedade do valor absoluto, temos que $\frac{1}{2}\left(Q_{i+1 / 2}^{n}+R a_{i+1 / 2}^{n}\right) \geq 0$, ou seja $D_{i+1 / 2}^{n} \geq 0$. Considerando a outra parte, temos que $\frac{1}{2}\left(-Q_{i+1 / 2}^{n}+R a_{i+1 / 2}^{n}\right) \leq 0$, o que resulta $C_{i+1 / 2}^{n} \geq 0$. Somando as duas constantes e usando a hipótese, temos que $C_{i+1 / 2}^{n}+D_{i+1 / 2}^{n}=Q_{i+1 / 2}^{n} \leq 1$. Pela proposição (3.3.35) o esquema é TVD incremental.

Observação 3.3.37. Se escrevermos o esquema de Lax-Friedrichs na forma- $Q$ com $Q_{i+1 / 2}^{n}=$ 1 e aplicarmos a Proposiçâo 3.3.36, vemos que esse esquema será TVD se $R\left|a_{i+1 / 2}^{n}\right| \leq 1$, ou seja, se a condiçâo CFL for satisfeita.

A Proposiçāo 3.3 .35 é um resultado importante e útil devido à condição (3.139) caracterizar a classe de esquemas incrementalmente TVD conservativos de três-pontos. Esta caracterização pode ser usada para provar o seguinte resultado.

Proposição 3.3.38. Um esquema conservativo de três-pontos que é incrementalmente TVD é no máximo de primeira ordem de precisão.

Demonstração: Considere um esquema na forma-Q

$$
u_{i}^{n+1}=u_{i}^{n}-\frac{R}{2} \delta_{0} G_{i}^{n}+\frac{1}{2} \delta_{+}\left(Q_{i-1 / 2}^{n} \delta_{-} u_{i}^{n}\right) .
$$


Como (3.142) é um esquema de três-pontos, notamos que o coeficiente de viscosidade numérica $Q_{i+1 / 2}^{n}$ pode depender somente de $u_{i}^{n}$ e $u_{i+1}^{n}$ ( embora

$$
\delta_{+}\left(Q_{i-1 / 2}^{n} \delta_{-} u_{i}^{n}\right)=Q_{i+1 / 2}^{n} \delta_{-} u_{i+1}^{n}-Q_{i-1 / 2}^{n} \delta_{-} u_{i}^{n}
$$

vai depender de $u_{i-1}^{n}, u_{i}^{n}$ e $u_{i+1}^{n}$ ). Retornamos à proposição 3.3 .29 e notamos que para um esquema da forma (3.142),

$$
W_{1}(u, u)=\left\{-\frac{R}{2} G^{\prime}\left(u_{i+1}\right)+\frac{1}{2}\left[Q\left(u_{i}, u_{i+1}\right)+Q_{2}\left(u_{i}, u_{i+1}\right)\left(u_{i+1}-u_{i}\right)\right]\right\}
$$

( onde $u_{i}=u_{i+1}=u$ )

$$
\begin{aligned}
& =-\frac{R}{2} G^{\prime}(u)+\frac{1}{2} Q(u, u) \\
W_{-1}(u, u) & =\left\{-\frac{R}{2} G^{\prime}\left(u_{i-1}\right)+\frac{1}{2}\left[Q\left(u_{i-1}, u_{i}\right)+Q_{-1}\left(u_{i-1}, u_{i}\right)\left(u_{i}-u_{i-1}\right)\right]\right\}
\end{aligned}
$$

( onde $u_{i-1}=u_{i}=u$ )

$$
\begin{aligned}
& =\frac{R}{2} G^{\prime}(u)+\frac{1}{2} Q(u, u) \quad \mathrm{e} \\
q(u) & =\frac{1}{2 R^{2}} Q(u, u)-\frac{1}{2}\left[G^{\prime}(u)\right]^{2} .
\end{aligned}
$$

Se o esquema (3.142) for de segunda ordem de precisão, devemos ter $q(u)=0$ ou $Q(u, u)=$ $R^{2}\left[G^{\prime}(u)\right]^{2}$. Pela Proposição 3.3 .36 , temos

$$
R\left|a_{i+1 / 2}^{n}\right| \leq Q_{i+1 / 2}^{n} \leq 1
$$

Se $a_{i+1 / 2}^{n}=G^{\prime}\left(u_{i}^{n}\right)$, então como $Q=R^{2} G^{2}=R\left|G^{\prime}\right| R\left|G^{\prime}\right| \leq Q R\left|G^{\prime}\right| \leq Q$ implica que $R\left|G^{\prime}\left(v_{i}^{n}\right)\right|=1$. Se $a_{i+1 / 2}^{n}=\delta_{+} G_{i}^{n} / \delta_{+} u_{i}^{n}$, então para $\delta_{+} u_{i}^{n}$ suficientemente pequeno $(\Delta x$ suficientemente pequeno) devemos ter

$$
R\left|\frac{\delta_{+} G\left(v_{i}^{n}\right)}{\delta_{+} v_{i}^{n}}\right|=1
$$

onde $v=v(x, t)$ é uma solução para a lei de conservação (3.98). Neste caso isto é uma contradição pois restringe $G$ ser igual ou aproximadamente igual a $1 / R$ vezes $u$, que certamente não é o caso (especialmente tendo em vista que $1 / R$ pode variar com $\Delta x$ e $\Delta t$ ).

Proposição 3.3.39. Em extremos suaves (máximos, mínimos ou pontos críticos) da solução que não são pontos sônicos, um esquema TVD incremental é no máximo de primeira ordem de precisão.

Demonstração: Seja $v=v(x, t)$ uma solução da lei de conservação (3.98) e sejam $C$ e $D$ as funções que definem $C_{i+1 / 2}^{n}$ e $D_{i-1 / 2}^{n}$, respectivamente, isto é, 


$$
\begin{aligned}
& C_{i+1 / 2}^{n}=C\left(u_{i-(p+1)}^{n}, \ldots, u_{i+q}^{n}\right) \\
& D_{i-1 / 2}^{n}=D\left(u_{i-(p+1)}^{n}, \ldots, u_{i+q}^{n}\right) .
\end{aligned}
$$

Observe que em geral $C_{i+1 / 2}^{n}$ e $D_{i-1 / 2}^{n}$. não irão ambos depender de $u_{i-(p+1)}^{n}, \ldots, u_{i+q}^{n}$. Entretanto, para esta demonstraçào é suficiente permitir que esta lista contenha todos os pontos sobre os quais $C_{i+1 / 2}^{n}$ e $D_{i-1 / 2}^{n}$ dependam, de maneira que possamos relacionar com a notação usada na definiçào de esquema de diferenças (3.64).

Continuando com a demonstração. para um esquema de diferenças na forma-I. o operador IV usado no esquema de diferenças (3.64) é:

$$
\Psi^{-}\left(u_{i-(p+1)}^{n}, \cdots, u_{i+q}^{n}\right)=u_{i}^{n}+C_{i+1 / 2}^{n} \delta_{+} u_{i}^{n}-D_{i-1 / 2}^{n} \delta_{-} u_{i}^{n} .
$$

Notamos que

$$
\begin{aligned}
& \sum_{j=-(p+1)}^{q} W_{j}\left(u_{i-(p+1)}, \ldots, u_{i+q}\right)=\sum_{j=-(p+1)}^{q} j^{2}\left[C_{j}\left(u_{i-(p+1)} \ldots u_{i+q}\right)\left(u_{i+1}-u_{i}\right)\right. \\
& \left.-D_{j}\left(u_{i-(p+1)}, \ldots, u_{i+q}\right)\left(u_{i}-u_{i-1}\right)\right]+C\left(u_{i-(p+1)}, \ldots, u_{i+q}\right)+D_{j}\left(u_{i-(p+1)}, \ldots, u_{i+q}\right) \text {. Usando }
\end{aligned}
$$$$
\text { o erro de truncamento dado na na Proposição 3.3.29 dado pelas equaçōes (3.124)-(3.125), }
$$
temos que

$$
q(u)=\frac{1}{2}\left[\frac{1}{R^{2}}\left(C(u, \ldots, u)+D(u, \ldots u)-\left(G^{\prime}(u)\right)^{2}\right)\right]
$$

e

$$
\begin{aligned}
\Delta t T_{i}^{n} & =-\Delta t^{2}\left\{\left[q(u) u_{x}\right]_{x}\right\}_{u=v_{i}^{n}}+o\left(\Delta t\left(\Delta t^{2}+\Delta x^{3}\right)\right) \\
& =-\Delta t^{2}\left\{q(u)_{x} u_{x}+q(u) u_{x x}\right\}_{u=r_{i}^{r}}+o\left(\Delta t\left(\Delta t^{2}+\Delta x^{3}\right)\right) .
\end{aligned}
$$

Como $v_{x}=0$ no extremo, temos

$$
\Delta t \tau_{i}^{n}=\frac{1}{2}\left[\frac{1}{R^{2}}\left(C\left(v_{i}^{n}, \ldots, v_{i}^{n}\right)+D\left(v_{i}^{n}, \ldots, v_{i}^{n}\right)-\left(G^{\prime \prime}\left(v_{i}^{n}\right)\right)^{2}\right)\right] .
$$

Para o esquema ser de segunda ordem de precisão, devemos ter

$$
C\left(v_{i}^{n}, \ldots, v_{i}^{n}\right)+D\left(v_{i}^{n}, \ldots, v_{i}^{n}\right)=R^{2}\left(G^{\prime}\left(v_{i}^{n}\right)\right)^{2} .
$$

A consistèıcia implica que

$$
C\left(v_{i}^{n}, \ldots, v_{i}^{n}\right)-D\left(v_{i}^{n}, \ldots, v_{i}^{n}\right)=-R G^{\prime}\left(v_{i}^{n}\right) .
$$


Resolvendo as equaçōes (3.147) e (3.148) para $C$ e $D$, temos

$$
C\left(v_{i}^{n}, \ldots, v_{i}^{n}\right)=\frac{1}{2}\left[R^{2}\left(G^{\prime}\left(v_{i}^{n}\right)\right)^{2}-R G^{\prime}\left(v_{i}^{n}\right)\right]
$$

e

$$
D\left(v_{i}^{n}, \ldots, v_{i}^{n}\right)=\frac{1}{2}\left[R^{2}\left(G^{\prime}\left(v_{i}^{n}\right)\right)^{2}+R G^{\prime}\left(v_{i}^{n}\right)\right] .
$$

Assumindo que o esquema satisfaz a condição CFL, $R\left|G^{\prime}\left(v_{i}^{n}\right)\right| \leq 1$, implica que

$$
C\left(v_{i}^{n}, \ldots, v_{i}^{n}\right) \leq 0 \text { ou } D\left(v_{i}^{n}, \ldots, v_{i}^{n}\right) \leq 0
$$

Das equações (3.149) e (3.150) vemos que a única maneira de $C\left(v_{i}^{n}, \ldots, v_{i}^{n}\right)$ ou $D\left(v_{i}^{n}, \ldots, v_{i}^{n}\right)$

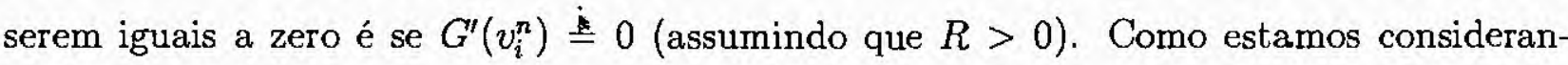
do somente pontos que não são sônicos, $G^{\prime}\left(v_{i}^{n}\right) \neq 0$, devemos ter $C\left(v_{i}^{n}, \ldots, v_{i}^{n}\right)<0$ ou $D\left(v_{i}^{n}, \ldots, v_{i}^{n}\right)<0$.

Usando expansão em série de Taylor, temos

$$
\begin{aligned}
C_{i+1 / 2} & =C\left(v_{i}^{n}, \ldots, v_{i}^{n}\right)+o(\Delta x) \\
D_{i-1 / 2} & =D\left(v_{i}^{n}, \ldots, v_{i}^{n}\right)+o(\Delta x) .
\end{aligned}
$$

Notando em (3.149) e (3.150) que $C\left(v_{i}^{n}, \ldots, v_{i}^{n}\right)$ e $D\left(v_{i}^{n}, \ldots, v_{i}^{n}\right)$ dependem de $G$ (e não de $\Delta x)$, quando combinados com (3.151) e (3.152), temos que

$$
C_{i+1 / 2}^{n}=-o(1)
$$

ou

$$
D_{i-1 / 2}^{n}=-o(1)
$$

Isto contradiz a hipótese de que o esquema de diferenças é incrementalmente TVD.

\subsubsection{Esquemas de Alta Resolução}

Nesta seção continuamos interessados em estudar esquemas conservativos que produzam uma solução que converge para a solução com viscosidade nula, e resolvam choques. Também iremos derivar uma classe de esquemas de diferenças TVD de alta ordem para a lei de conservação hiperbólica.

Esquemas de alta resoluçāo são usados para descrever esquemas que geralmente serão de segunda ordem de precisão (ou maior, embora a ênfase será para segunda ordem) sobre 
seções suaves da solução, não-oscilatórios e capazes de resolver precisamente descontinuidades na solução. Dos resultados apresentados na seção anterior, veremos que para encontrar um esquema de alta resolução, (i) devemos usar um esqucma não linear, (ii) tentar encontrar esquemas que não sejam de três-pontos, e (iii) o máximo que podemos esperar é que nossos esquemas sejam de segunda ordem de precisão fora dos pontos extremos da solução (que não sejam pontos sônicos da solução).

\subsubsection{Métodos Limitantes de Fluxo}

A idéia é construir um esquema de alta resolução cuja função de fluxo numérico seja definida em termos das funçōes de fluxo numérico de alguns dos esquemas anteriormente estudados. Em particular, escrevemos a função de fluxo numérico de nosso esquema como

$$
g_{i+1 / 2}^{n}=g_{\mathrm{L}_{i+1 / 2}}^{n}+\phi_{i}^{n}\left[g_{\mathrm{H}_{i+1 / 2}}^{n}-g_{\mathrm{L}_{i+1 / 2}}^{n}\right]
$$

onde $g_{\mathrm{L}_{i+1 / 2}}^{n}$ e $g_{\mathrm{H}_{i+1 / 2}}^{n}$ são funções de fluxo numérico de um esquema conservativo de ordem baixa e um de ordem alta, respectivamente, e $\phi_{i}^{n}$ será definido a seguir. O objetivo é desenvolver um esquema onde a função de fluxo numérico tenha a capacidade de suavização do esquema de baixa ordem e a precisão daquele de alta ordem quando isso for possível. Dentro desses princípios, definimos:

$$
\phi_{i}^{n} \cong \begin{cases}1 & \text { sobre seções suaves da solução, }\left(g_{i+1 / 2}^{n} \cong g_{\mathrm{H}_{i+1 / 2}}^{n}\right), \\ 0 & \text { sobre seções com altos gradientes ou descontinuidades, }\left(g_{i+1 / 2}^{n} \cong g_{L_{i+1 / 2}}^{n}\right) .\end{cases}
$$

Devemos notar que quando definimos (3.153), o esquema resultante automaticamente será conservativo. Se $g_{\mathrm{L}_{i+1 / 2}}^{n}$ e $g_{\mathrm{H}_{i+1 / 2}}^{n}$ são funções de fluxo numérico associadas com esquemas de três-pontos (os quais são mais fáceis de inicializar), o termo $\phi_{i}^{n}$ pode ser usado para obter um esquema com mais de três de pontos (veja Proposição 3.3.38). Também, a combinação de $g_{\mathrm{L}_{i+1 / 2}}^{n}, g_{\mathrm{H}_{i+1 / 2}}^{n}$ e $\phi_{i}^{n}$ pode resultar em um esquema não linear (Proposição 3.3.31).

E finalmente, também podemos facilmente escrever $g_{i+1 / 2}^{n}$ como

$$
g_{i+1 / 2}^{n}=g_{\mathrm{H}_{i+1 / 2}}^{n}-\left(1-\phi_{i}^{n}\right)\left[g_{\mathrm{H}_{i+1 / 2}}^{n}-g_{\mathrm{L}_{i+1 / 2}}^{n}\right]
$$




\section{Esquemas Limitantes de Fluxo para a Equação da Onda Linear}

Para simplificar o desenvolvimento e a análise desta classe de esquemas numéricos, seguindo Sweby em [42] começamos introduzindo uma classe de esquemas de alta resolução para a equação linear:

$$
v_{t}+a v_{x}=0
$$

Iniciamos escrevendo a função de fluxo numérico associada com o esquema de Lax-Wendroff (linear) para resolver (3.155) como

$$
g_{i+1 / 2}^{n}=a u_{i}^{n}+\frac{1}{2} a(1-a R) \delta_{+} u_{i}^{n} .
$$

A função de fluxo numérico (3.156) pode ser interpretada como $g=g_{\mathrm{L}}+\left(g_{\mathrm{H}}-g_{\mathrm{L}}\right)$ onde

$$
g_{\mathrm{L}_{i+1 / 2}}^{n}=a u_{i}^{n}
$$

e

$$
g_{\mathrm{H}_{i+3 / 2}}^{n}=a u_{i}^{n}+\frac{1}{2} a(1-a R) \delta_{+} u_{i}^{n} .
$$

A função $g_{\mathrm{L}}$ é a função de fluxo numérico associada com o esquema de primeira ordem FTBS, e, $g_{\mathrm{H}}$ é a função associada com o esquema de Lax-Wendroff. Como o esquema FTBS é instável quando $a<0$, iremos assumir que $a>0$.

Continuamos nossa estratégia para construir esquemas de alta resolução substituindo a função de fluxo numérico (3.156) por

$$
g_{i+1 / 2}^{n}=g_{\mathbf{L}_{i+1 / 2}}^{n}+\phi_{i}^{n}\left[g_{\mathbf{H}_{i+1 / 2}}^{n}-g_{\mathbf{L}_{i+1 / 2}}^{n}\right]=a u_{i}^{n}+\phi_{i}^{n} \frac{1}{2} a(1-a R) \delta_{+} u_{i}^{n} .
$$

A função $\phi_{i}^{n}$ é chamada limitante de fluxo. Claramente, a função de fluxo numérico (3.159) é obtida da combinação, $g=g_{\mathrm{L}}+\phi\left(g_{\mathrm{H}}-g_{\mathrm{L}}\right)$, onde $g_{\mathrm{L}}$ e $g_{\mathrm{H}}$ são as funçôes de fluxo numérico dos esquemas FTBS e Lax-Wendroff, respectivamente. No caso de (3.159), como já mencionamos antes, é desejável que $\phi_{i}^{n}$ se aproxime de 1 em regiões onde a solução é suave e se aproxime de 0 próximo das descontinuidades, pois assim estaremos usando um esquema de segunda ordem nas regiōes suaves e um esquema upwind nas descontinuidades. É desejável que a função $\phi$ seja capaz de "localizar"a descontinuidade. Existem muitas maneiras para 
definir $\phi_{i}^{n}$ afim de alcançarmos nossos objetivos. Uma maneira é escrever $\phi_{i}^{n}=\phi\left(\theta_{i}^{n}\right)$ onde $\theta_{i}^{n}$ é conhecido como uma medida de suavidade e pode ser definido por:

$$
\theta_{i}^{n}=\frac{\delta_{-} u_{i}^{n}}{\delta_{+} u_{i}^{n}}
$$

Embora a função $\phi$ ainda esteja indeterminada, notamos que $\theta_{i}^{n}$ é usado para medir a suavidade da solução, pois quando $\theta_{i}^{n}$ se aproxima de 1 o seu gradiente não muda muito e se for muito diferente de 1 , entào o gradiente muda rapidamente. $O$ esquema de diferenças a ser considerado é:

$$
\begin{aligned}
u_{i}^{n+1} & =u_{i}^{n}-R\left[g_{i+1 / 2}^{n}-g_{i+1 / 2}^{n}\right] \\
& =u^{n}-a R \delta_{-} u_{i}^{n}-\frac{1}{2} a R(1-a R) \phi_{i}^{n} \delta_{+} u_{i}^{n}+\frac{1}{2} a R(1-a R) \phi_{i-1}^{n} \delta_{+} u_{i-1}^{n} \\
& =u_{i}^{n}-a R \delta_{-} u_{i}^{n}-\frac{1}{2} a R(1-a R) \delta_{-}\left(\phi_{i}^{n} \delta_{+} u_{i}^{n}\right) .
\end{aligned}
$$

Observação 3.3.40. Diferentes escolhas para $\phi$ em (3.162), fornece diferentes esquemas. Para $\phi(\theta)=0$ temos o esquema FTBS, para $\phi(\theta)=1$ Lax-Wendroff e $\phi(\theta)=\theta$ temos o esquema de Beam-Warming.

O objetivo é escolher $\phi$ de tal maneira que o esquema resultante seja TVD e de segunda ordem (exceto nos pontos de máximos e mínimos da solução que não são pontos sônicosProposiçâo 3.3.39). A estratégia que usaremos para assegurar que nosso esquema é TVD será exigir que ele seja incrementalmente TVD e usar a Proposição 3.3.35. Isto é equivalente A escrever o esquema na forma incremental e usar a Proposição 3.3 .23 para forçar o esquema a ser TVD. O esquema (3.161) na forma-I torna-se

$$
u_{i}^{n+1}=u_{i}^{n}-\left[a R-\frac{1}{2} a R(1-a R) \phi_{i-1}^{n}\right] \delta_{-} u_{i}^{n}-\frac{1}{2} a R(1-a R) \phi_{i}^{n} \delta_{+} u_{i}^{n}
$$

com

$$
C_{i+1 / 2}^{n}=-\frac{1}{2} a R(1-a R) \phi_{i}^{n}
$$

e

$$
D_{i-1 / 2}^{n}=a R-\frac{1}{2} a R(1-a R) \phi_{i-1}^{n} .
$$

É claro que quando $0 \leq a R \leq 1$ (condição CFL) e $\phi_{i}^{n}$ está próximo de 1 (nas partes suaves da solução), $C_{i+1 / 2}^{n}$ vai ser negativo, e portanto, o esquema (3.163) nào será TVD incremental. 
A revelação acima não é um defeito crucial, pois, podemos reescrever (3.161) na forma-I como

$$
u_{i}^{n+1}=u_{i}^{n}-\left\{a R-\frac{1}{2} a R(1-a R) \phi_{i-1}^{n}+\frac{1}{2} a R(1-a R) \phi_{i}^{n} \frac{\delta_{+} u_{i}^{n}}{\delta_{-} u_{i}^{n}}\right\} \delta_{-} u_{i}^{n} .
$$

Assim temos que

$$
C_{i+1 / 2}^{n}=0
$$

e

$$
D_{i-1 / 2}^{n}=a R-\frac{1}{2} a R(1-a R) \phi_{i-1}^{n}+\frac{1}{2} a R(1-a R) \phi_{i}^{n} \frac{\delta_{+} u_{i}^{n}}{\delta_{-} u_{i}^{n}}
$$

A discussão acima revela um fato muito importante: que a forma-I de um esquema de diferenças não é única. As condições (3.116) são então satisfeitas se

$$
0 \leq D_{i-1 / 2}^{n} \leq 1
$$

Reescrevendo $D_{i-1 / 2}^{n}$ como

$$
D_{i-1 / 2}^{n}=a R-\frac{1}{2} a R(1-a R) \phi\left(\theta_{i-1}^{n}\right)+\frac{1}{2} a R(1-a R) \frac{\phi\left(\theta_{i}^{n}\right)}{\theta_{i}^{n}},
$$

e se $\phi$ satisfaz

$$
\left|\frac{\phi\left(\theta_{i}\right)}{\theta_{i}}-\phi\left(\theta_{i-1}\right)\right| \leq 2 \quad \forall i
$$

então a desigualdade (3.166) é satisfeita.

Iremos agora usar a desigualdade (3.168) para auxiliar na escolha da forma da função limitadora $\phi$. Para que $\phi$ seja não-negativa; assumimos que $\phi(\theta)=0$ para $\theta \leq 0$. Notamos que a desigualdade (3.168) será satisfeita se exigirmos que $\phi$ satisfaça

$$
0 \leq \frac{\phi(\theta)}{\theta} \leq 2 \quad \text { e } \quad 0 \leq \phi(\theta) \leq 2 \quad \forall \theta
$$

Assim, escolhemos a função $\phi$ de tal maneira que seu gráfico esteja no interior da região da Figura 3.3.9.

Observação 3.3.41. Vimos que se escrevermos o esquema de diferenças (3.162) na formaI como em (3.163), o esquema não será incrementalmente TVD. Reescrevendo o esquema como em (3.165) permite-nos provar que o esquema é incrementalmente TVD. O esquema de differenças não muda. Esta observação mostra que a propriedade de ser incrementalmente TVD é dependente da representação da formar $I$. 
Existem obviamente muitas funções que podem ser escolhidas de tal maneira que o gráfico esteja contido na região hachurada da Figura 3.3.9. O resultado seguinte nos fornece algumas propriedades gerais do esquema de diferenças (3.162).

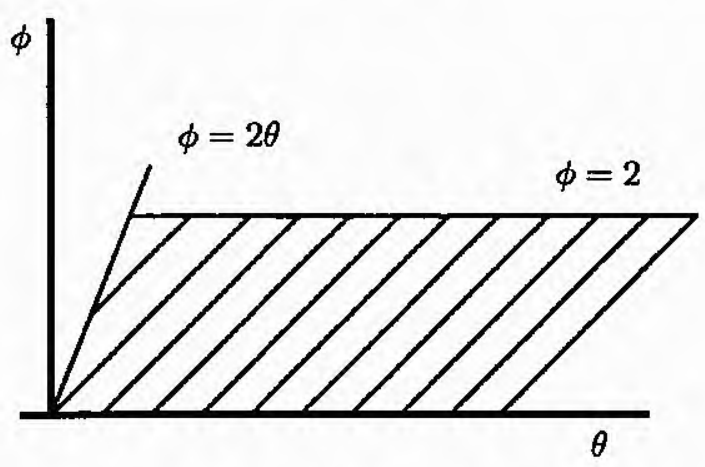

Figura 3.3.9. A região hachurada representa a região definida pela desigualdade (3.169) para $\theta \geq 0$. (Região TVD)

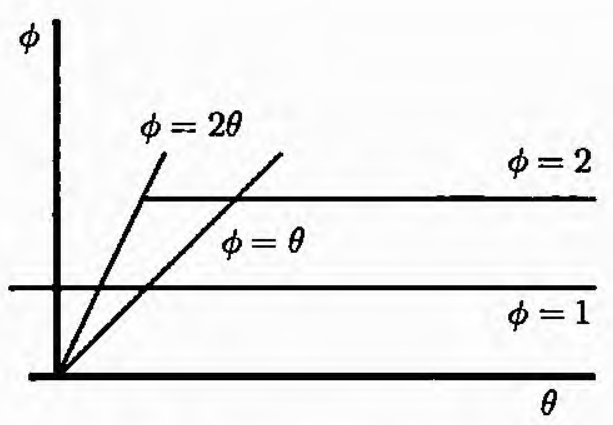

Figura 3.3.10. Gráficos das funções associadas com o esquema de Lax-Wendroff ( $\phi=1)$ e o esquema de Beam-Warming $(\phi=\theta)$.

Proposição 3.3.42. Se a função limitante de fluxo $\phi$ é limitada, entâo o esquema de diferenças (3.162) é consistente com a equação da onda unidimensional (3.155). Se $\phi(1)=1 e$ $\phi$ é Lipschitz contínua em $\theta=1$, então o esquema de diferenças (3.162) é de segunda ordem para soluções suaves com $\left|v_{x}\right| \gg>$.

Demonstração: A primeira parte do esquema (3.162), isto é, $\left(u_{i}^{n+1}=u_{i}^{n}-a R\left(u_{i}^{n}-u_{i-1}^{n}\right)\right)$, é consistente com (3.155). O último termo de (3.162) é um termo de segunda ordem (primeira ordem quando dividido por $\Delta t$ ). Portanto, a consistência de (3.162) está garantida.

Para provar a segunda afirmação, vamos utilizar a Proposição 3.3.29 que exige que $\phi(\theta)$ seja diferenciável em $\theta=1$, hipótese mais forte que a da Proposição 3.3.42. No entanto a prova nas condições da Proposição 3.3.42 é uma variação técnica daquela quando assumimos $\phi$ diferenciável. Apresentamos esta última versão. Para a aplicação da Proposição 3.3.29, 
escrevemos nosso esquema de diferenças na forma

$$
\begin{aligned}
u_{i}^{n+1} & =W\left(u_{i-2}^{n}, \ldots, u_{i+1}^{n}\right) \\
& =u_{i}^{n}-a R \delta_{-} u_{i}^{n}-\frac{1}{2} a R(1-a R) \delta_{-}\left(\phi_{i}^{n} \delta_{+} u_{i}^{n}\right)
\end{aligned}
$$

onde $p=q=1$ e salientamos que $\phi_{i}^{n}$ e $\phi_{i-1}^{n}$ dependem de $u_{i-1}^{n}, u_{i}^{n}, u_{i+1}^{n}$ e $u_{i-2}^{n}, u_{i-1}^{n}, u_{i}^{n}$, respectivamente. Um cálculo simples mostra que

$$
q(u)=\frac{1}{2}\left[\frac{1}{R^{2}}(a R-a R(1-a R) \phi(1))-\left(G^{\prime}(u)\right)^{\dot{2}}\right] .
$$

Como para a lei de conservação $(3.155) G^{\prime}(u)=a$, é fácil ver que quando $\phi(1)=1, q(u)=0$. Como $q=0$ para todo $u$, temos que $q^{\prime}=0$ para todo $u$, e pela Proposição 3.3 .29 o esquema de diferenças é no mínimo de segunda ordem de precisão.

Nosso objetivo será descobrir funções $\phi$ cujos gráficos estejam contidos na região hachurada da Figura 3.3.9 e passem pelo ponto $(1,1)$. Na Figura 3.3 .10 vemos os gráficos das funções limitadoras, $\phi(\theta)=1$ associada com o esquema de Lax-Wendroff e $\phi(\theta)=\theta$ associada com o esquema de Beam-Warming. Embora ambas passem pelo ponto $(1,1)$, (já sabemos que são métodos de segunda ordem) seus gráficos não estão completamente contidos na região (TVD) (também sabemos que não são esquemas TVD). Analisando a expressão (3.160) vemos que para $\theta$ grande ( $\delta_{+} u_{i}^{n}$ deve ser pequeno e $\delta_{-} u_{i}^{n}$ não deve ser pequeno), o esquema de Beam-Warming não é TVD. Similarmente, quando $\theta$ é pequeno, o esquema de Lax-Wendroff também não é TVD. Como todas as situações descritas acima ocorrem próximas de descontinuidades na solução, esses esquemas deixam de ser TVD nas proximidades das descontinuidades. As oscilações na solução numérica observadas próximo das descontinuidades (utilizando o esquema de Lax-Wendroff) causam aumento na variação da solução numérica o que tornam esses esquemas não TVD.

Uma possível escolha para $\phi$ é fazê-la de modo que o fluxo antidifusivo $\left(\phi_{i}^{n}\left[g_{\mathrm{H}_{i+1 / 2}}^{n}\right.\right.$ $\left.\left.g_{\mathrm{L}_{i+1 / 2}}^{n}\right]\right)$ que adicionamos no esquema de primeira ordem seja maximizado, ou seja

$$
\phi(\theta)=\min \{2 \theta, 2\}, \quad \theta>0 .
$$

Notamos que o gráfico desta função é o limite superior da região TVD da Figura 3.3.9. Entretanto, como $\phi(1) \neq 1$, este limitador não nos fornecerá um esquema de segunda ordem.

Exibimos abaixo algumas das principais funções limitadoras apresentadas e discutidas na literatura. 
Funçāo Limitadora Superbee, [42]

$$
\phi(\theta)=\max \{0, \min \{1,2 \theta\}, \min \{\theta, 2\}\}
$$

Função Limitadora de Van Leer, [42]

$$
\phi(\theta)=\frac{|\theta|+\theta}{1+|\theta|}
$$

Função Limitadora de Chakravarthy-Osher-(CO), [42]

$$
\phi(\theta)=\max \{0, \min \{\theta, \psi\}\}, \quad 1 \leq \psi \leq 2
$$

Função Limitadora de Beam-Warming-Lax-Wendroff-(BW-LW), [44]

$$
\phi(\theta)=\max \{0, \min \{\theta, 1\}\}
$$

Observação 3.3.43. Observamos que o limitador $B W-L W$ (combinação dos esquemas de Beam-Warming e de Lax-Wendroff) é um caso especial do limitador $C-O(\psi=1)$. Também, que as funções limitadoras (3.170)-(3.173) satisfazem a condição (3.169).

É claro que existem muitas outras possíveis funçôes limitadoras. Em [42], Sweby afirma que é melhor escolher a função limitadora de tal maneira que a função de fluxo numérico seja uma média ponderada das funções de fluxo de Lax-Wendroff e de Beam-Warming. Se fizermos assim, veremos que o gráfico da função limitadora estará contido na região hachurada da Figura 3.3.11. Notamos que o gráfico de qualquer função limitadora contido nessa região deve satisfazer $\phi(1)=1$ e que os gráficos das funções (3.170)-(3.173) estão na região da Figura 3.3.11.

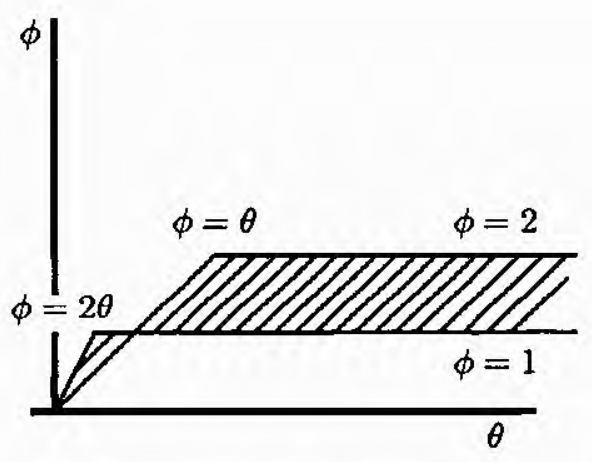

Figura 3.3.11. Gráfico da região representando funções limitadoras que são médias ponderada das funções de Lax-Wendroff e de Beam-Warming. (Região TVD de segunda ordem) 


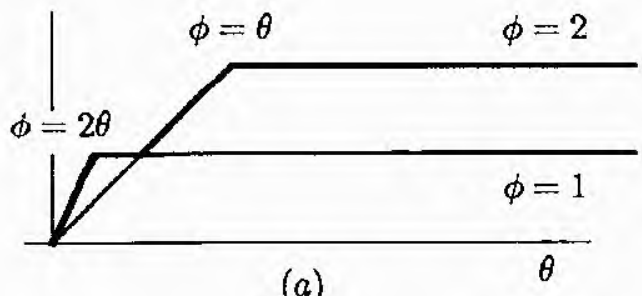

(a)

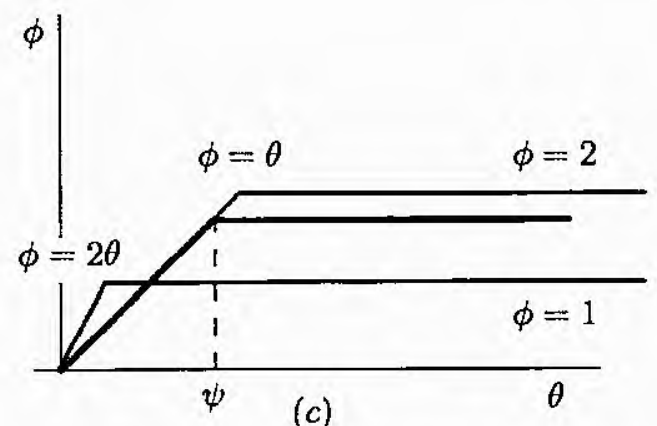

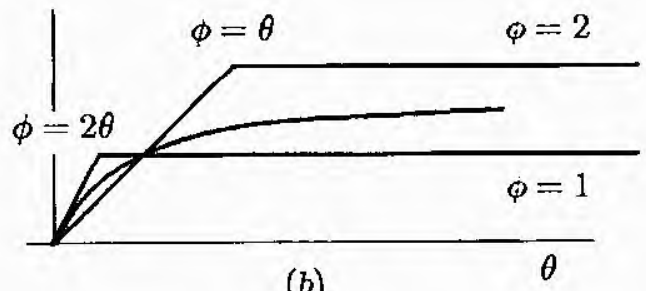

(b)

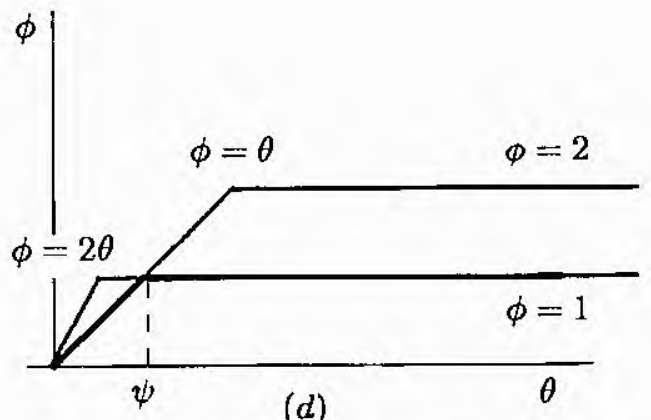

(d)

Figura 3.3.12. Gráficos das funçòes limitantes de (a) Superbee, (b) Van Leer, (c) C-O, (d) BW-LW.

Podemos notar que todas as funções limitadoras que apresentamos são funções crescentes monótonas. Outra propriedade que algumas dessas funções possuem é a simetria. Dizemos que uma função limitadora é simétrica se

$$
\frac{\phi(\theta)}{\theta}=\phi\left(\frac{1}{\theta}\right)
$$

A propriedade de simetria da função limitadora assegura que o esquema (3.162) será preciso no tratamento dos gradientes, independente da direção de propagação das características. Os limitadores, Superbee, Van Leer (veja Figura 3.3.13) e BW-LW, por exemplo, são simétricos, enquanto que para $\psi>1$ o limitador $\mathrm{C}$-O não é simétrico, conforme mostrado na Figura 3.3.13. A diferença entre as funções limitadoras não simétricas e as simétricas é ilustrada resolvendo-se o problema:

$$
\begin{aligned}
& v_{t}-v_{x}=0, \quad x \in(0,1), t>0 \\
& v(x, 0)=f(x), \quad x \in[0,1] \\
& v(0, t)=v(1, t), \quad t \geq 0
\end{aligned}
$$

onde $f$ é definida por

$$
f(x)= \begin{cases}1 & \text { se } 0.4 \leq x \leq 0.6 \\ 0 & \text { caso contrário. }\end{cases}
$$


Toda a análise apresentada até aqui é válida para $a>0$ e pode ser repetido para $a<0$. Quando $a<0$ obtemos a seguinte função de fluxo numérico

$$
g_{i+1 / 2}^{n}=a u_{i+1}^{n}-\phi_{i}^{n} \frac{1}{2} a(1+a R) \delta_{+} u_{i}^{n}
$$

onde $\phi_{i}^{n}=\phi\left(\theta_{i}^{n}\right)$ e $\theta_{i}^{n}=\delta_{+} u_{i+1}^{n} / \delta_{+} u_{i}^{n}$.

Compondo os esquemas para $a>0$ e $a<0$, obtemos o esquema

$$
g_{\mathrm{L}_{i+1 / 2}}^{n}=\frac{1}{2} a\left(u_{i}^{n}+u_{i+1}^{n}\right)-\frac{1}{2}|a|\left(u_{i+1}^{n}-u_{i}^{n}\right)
$$

que não faz restrição no sinal de $a$. A função de fluxo numérico (3.180) fornece o esquema FTBS quando $a>0$ e o esquema FTFS quando $a<0$. Então, usando o esquema de LaxWendroff como o esquema de alta ordem, obtemos um esquema de alta ordem com função de fluxo numérico

$$
g_{i+1 / 2}^{n}=g_{\mathrm{L}_{i+1 / 2}}^{n}+\frac{1}{2} \phi_{i}^{n} \vec{a}(\operatorname{sign}(a)-a R) \delta_{+} u_{i}^{n}
$$

onde $\operatorname{sign}(a)$ denota o sinal de $a, g_{\mathrm{L}_{i+1 / 2}}^{n}$ é dado por (3.180), e

$$
\theta_{i}^{n}= \begin{cases}\frac{\delta_{-} u_{i}^{n}}{\delta+u_{i}^{n}} & \text { quando } a>0 \\ \frac{\delta_{+} u_{i+1}^{n}}{\delta_{+} u_{i}^{n}} & \text { quando } a<0\end{cases}
$$

É fácil ver que a função de fluxo numérico (3.181) reduz-se às funções de fluxo numérico (3.179) e (3.159) quando $a<0$ e $a>0$, respectivamente.
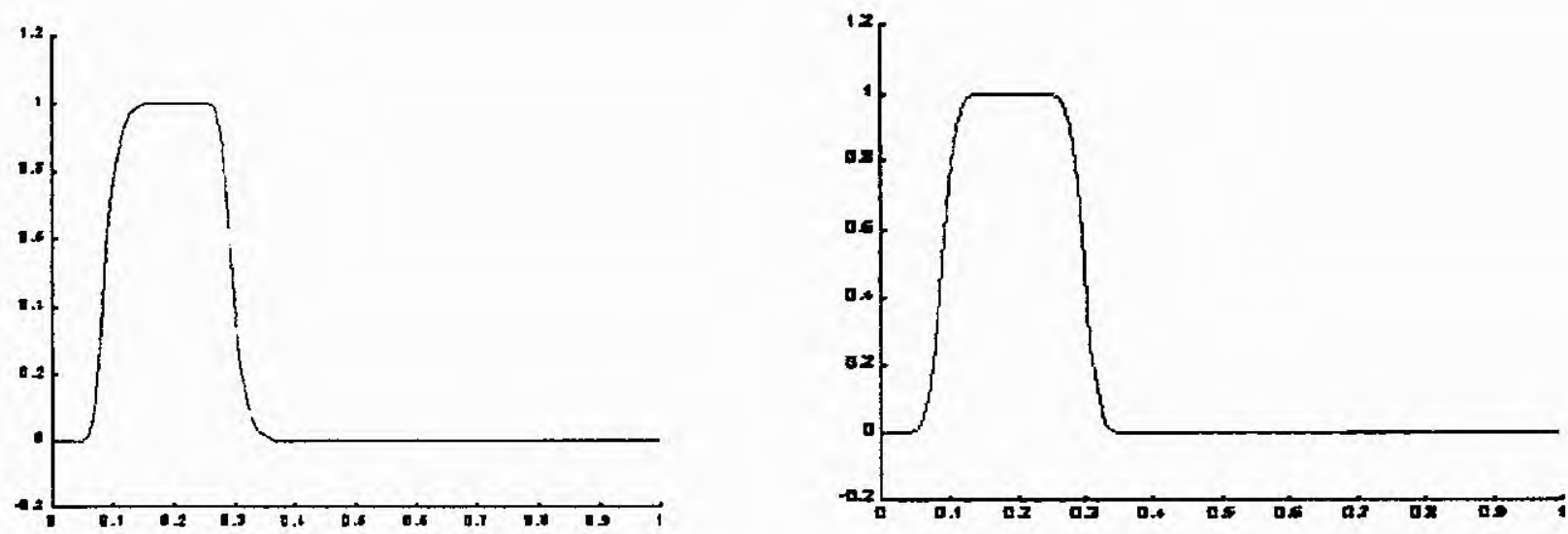

Figura 3.3.13. Solução aproximada para o problema de valor inicial (3.175)-(3.178). A solução foi encontrada usando a função limitadora C-O (não simétrica) e de Van-Leer (simétrica), respectivamente, para $\Delta x=0.01, \Delta t=0.005$, no tempo $t=0.3$. 
O objetivo até agora foi o de encontrar esquemas com as três propriedades básicas: conservativos, TVD, e que forneçam soluções entrópicas. Atéo momento derivamos esquemas de alta resolução que são TVD e conservativos. Infelizmente não é possível provar que os esquemas de alta resolução desenvolvidos nesta seção fornecem soluções entrópicas. Evidências numéricas indicam que as soluções obtidas usando esquemas limitadores de fluxo são entrópicas. Na referência [42], Sweby conjecturou que os esquemas limitadores de fluxo fornecem as soluções entrópicas pois eles herdam essa propriedade dos esquemas de baixa ordem.

\section{Esquemas Limitantes de Fluxo para Leis de Conservação Não Lineares}

Iremos nessa seção estender as idéias desenvolvidas para a equação de advecção linear na seção anterior, para aproximar soluções para a lei de conservação não linear (3.98). Análogo ao que foi feito anteriormente, construimos uma função de fluxo numérico da forma

$$
g_{i+1 / 2}^{n}=g_{\mathrm{L}_{i+1 / 2}}^{n}+\phi_{i}^{n}\left[g_{\mathrm{H}_{i+1 / 2}}^{n}-g_{\mathrm{L}_{i+1 / 2}}^{n}\right]
$$

A analogia mais óbvia com a seção anterior é usar esquemas upwind não lineares (esquemas de diferenças (3.54)) como nosso esquema de baixa ordem e o esquema não linear de Lax Wendroff (esquema de diferenças (3.93)) como esquema de alta ordem. Portanto, sejam

$$
g_{\mathrm{L}_{i+1 / 2}}^{n}=\frac{1}{2}\left[G_{i}^{n}+G_{i+1}^{n}\right]-\frac{1}{2}\left|a_{i+1 / 2}^{n}\right| \delta_{+} u_{i}^{n}
$$

e

$$
g_{\mathrm{H}_{i+1 / 2}}^{n}=\frac{1}{2}\left[G_{i}^{n}+G_{i+1}^{n}\right]-\frac{R}{2}\left(a_{i+1 / 2}^{n}\right)^{2} \delta_{+} u_{i}^{n}
$$

as funções de fluxo numérico desses esquemas, combinando-as obtemos um esquema de diferenças com função de fluxo numérico

$$
g_{i+1 / 2}^{n}=g_{\mathbf{L}_{i+1 / 2}}^{n}+\phi_{i}^{n} \frac{\left|a_{i+1 / 2}^{n}\right|}{2}\left[1-R\left|a_{i+1 / 2}^{n}\right|\right] \delta_{+} u_{i}^{n} .
$$

Observação 3.3.44. A Figura 3.3.14 abaixo, destaca a eficiência do esquema limitante de fluxo (3.185) em comparação com o esquema de Lax-Wendroff (3.90). A correção do método de Lax-Wendroff visa eliminar as oscilações que esse método possui no entorno das descontinuidades, conforme mostrado pela solução pontilhada. O contraste entre as duas soluções é significativo porque o método (3.185) utiliza um esquema de ordem 1 (correçâo) 
o que não gera oscilações pois o termo difusivo presente neste método é grande o suficiente para suavizar a solução.

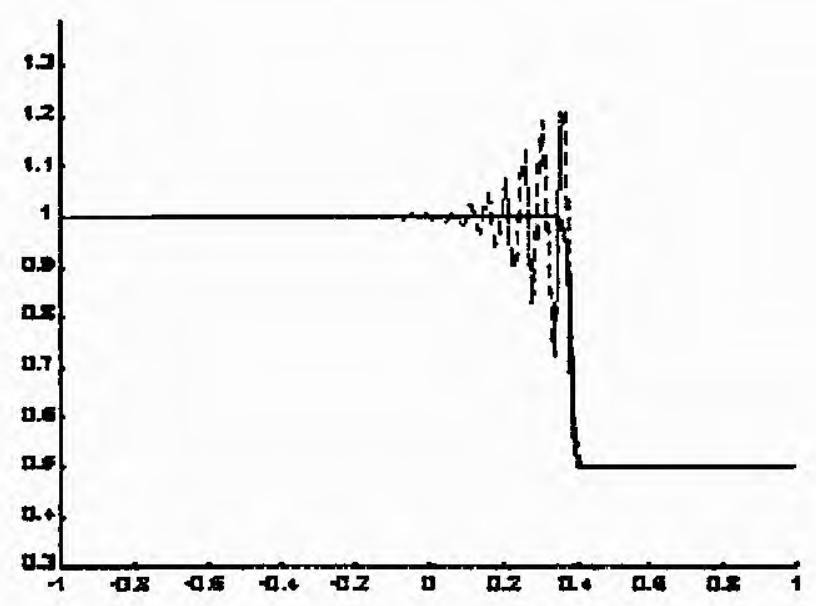

Figura 3.3.14. Solução da equação de Burgers com condição inicial (3.97), utilizando o esquema (3.185), a função limitadora de C-O (3.172), $\operatorname{com} \psi=2, \Delta x=0.01, \Delta t=0.001$, no tempo $t=0.5$.

Como estamos usando um esquema upwind para o esquema de baixa ordem, usaremos uma definição do parâmetro de suavidade $\theta_{i}^{n}$ similar àquela dada em (3.182):

$$
\theta_{i}^{n}= \begin{cases}\frac{\delta-u_{i}^{n}}{\delta+u_{i}^{n}} & \text { quando } a_{i+1 / 2}^{n}>0 \\ \frac{\delta_{+} u_{i+1}^{n}}{\delta_{+} u_{i}^{n}} & \text { quando } a_{i+1 / 2}^{n}<0\end{cases}
$$

Enfatizamos que o novo esquema (3.185) vai herdar a condição CFL dos esquemas de baixa e alta ordem. Por esta razão é aconselhável escolher ambos os esquemas com condições CFL compatíveis. Se por exemplo usarmos os esquemas não lineares FTFS ou FTBS como esquemas de baixa ordem, deveríamos ter um esquema de alta or dem com uma condição CFL restritiva. Além do mais, podemos construir e escolher esquemas monótonos ou esquemasE como os de menor ordem e qualquer outro esquema de alta ordem, embora não temos muitas opções. Por exemplo, vamos apresentar um esquema composto pelos esquemas de Lax-Friedrichs e de Beam-Warming.

$$
g_{i+1 / 2}^{n}=\frac{1}{2}\left[G_{i}^{m}+G_{i+1}^{m}\right]-\frac{1}{2 R} \delta_{+} u_{i}^{n}+\phi_{i}^{n}\left\{\frac{1}{2}\left[G_{i}^{*}-G_{i+1}^{n}\right]+\frac{1}{2} \delta_{-} G_{i}^{n}+\frac{1}{2 R} \delta_{+} u_{i}^{n}\right\}
$$

onde $u_{i}^{*}=u_{i}^{n}-R \delta_{-} G_{i}^{n}$ e o parâmetro de suavização é definido como em (3.186). Uma desvantagem do esquema (3.187) acima, é que embora o limitador "olhe"em ambas as direções para ver como a solução está mudando ele não reage muito ao que "vê". O valor de $\phi_{i}^{n}$ é a única 
grandeza que muda. É possível, e algumas vezes vantajoso, incluir termos diferentes na função de fluxo numérico especialmente projetados para tratar as informações vindas da esquerda c da direita.

$\mathrm{Na}$ referência [42] o autor sugeriu um esquema com a seguinte função de fluxo numérico:

$$
\begin{aligned}
g_{i+1 / 2}^{n}= & g_{i+1 / 2}^{\mathrm{E}}-\frac{1}{2} \phi\left(\theta_{i}^{+}\right)\left\{\left(g_{i+1 / 2}^{\mathrm{E}}-G_{i+1}^{n}\right)+\frac{R}{\delta_{+} u_{i}^{n}}\left(g_{i+1 / 2}^{\mathrm{E}}-G_{i+1}^{n}\right)^{2}\right\} \\
& -\frac{1}{2} \phi\left(\theta_{i-1}^{-}\right)\left\{\left(g_{i+1 / 2}^{\mathrm{E}}-G_{i}^{n}\right)+\frac{R}{\delta_{+} u_{i}^{n}}\left(g_{i+1 / 2}^{\mathrm{E}}-G_{i}^{n}\right)^{2}\right\}
\end{aligned}
$$

onde $\theta_{i}^{+}$e $\theta_{i-1}^{-}$são dados por:

$$
\begin{aligned}
\theta_{i}^{+} & =\frac{\delta_{+} u_{i}^{n}}{\delta_{+} u_{i-1}^{n}} \frac{\left[\delta_{+} u_{i-1}^{n}+R\left(g_{i-1 / 2}^{\mathrm{E}}-G_{i}^{n}\right)\right]\left(g_{i-1 / 2}^{\mathrm{E}}-G_{i}^{n}\right)}{\left[\delta_{+} u_{i}^{n}+R\left(g_{i+1 / 2}^{\mathrm{E}}-G_{i+1}^{n}\right)\right]\left(g_{i+1 / 2}^{\mathrm{E}}-G_{i+1}^{n}\right)} \\
\theta_{i-1}^{-} & =\frac{\delta_{+} u_{i-2}^{n}}{\delta_{+} u_{i-1}^{n}} \frac{\left[\delta_{+} u_{i-1}^{n}+R\left(g_{i-1 / 2}^{\mathrm{E}}-G_{i-1}^{n}\right)\right]\left(g_{i-1 / 2}^{\mathrm{E}}-G_{i-1}^{n}\right)}{\left[\delta_{+} u_{i-2}^{n}+R\left(g_{i-3 / 2}^{\mathrm{E}}-G_{i-2}^{n}\right)\right]\left(g_{i-3 / 2}^{\mathrm{E}}-G_{i-2}^{n}\right)} .
\end{aligned}
$$

Nesta função são incluidos os termos de fluxo antidifusivo positivo e negativo, projetados para tratar as informaçōes que estão propagando à direita e à esquerda, respectivamente.

Quando $G(v)=a v, a>0$, e $g_{i+1 / 2}^{\mathbf{E}}=a u_{i}^{n}$, o esquema de diferenças definido pela função de fluxo numérico (3.188) com $\theta$ definido por (3.189) e (3.190) reduz-se ao esquema (3.162). Por outro lado, quando $G(v)=a v, a<0$, e $g_{i+1 / 2}^{\mathrm{E}}=a u_{i+1}^{n}$, esse mesmo esquema reduz-se ao esquema (3.179) com $\theta_{i}^{n}=\delta_{+} u_{i+1}^{n} / \delta_{+} u_{i}^{n}$. É possível ver também que quando o esquema de baixa ordem é escolhido como um upwind não linear $\left(g_{i-1 / 2}^{n}=G_{i}^{n}\right.$ ou $\left.g_{i-1 / 2}^{n}=G_{i-1}^{n}\right)$, um dos parâmetros de suavização $\theta^{ \pm}$é zero em todo ponto. Neste caso, em um dado ponto da malha o esquema determina de que direção a informação está se aproximando e usa o termo apropriado em (3.188) para tratar da direção correta. Sweby em [42] mostrou que se a condição $C F L, R\left|G^{\prime}\right| \leq \frac{2}{3}$ é satisfeita, então o esquema de diferenças (3.188)-(3.190) é TVD.

Ainda no intuito de encontrar esquemas TVD de segunda ordem, derivamos a seguir o método limitante de inclinação.

\subsubsection{Métodos Limitante de Inclinação}

$\mathrm{Na}$ Seção 3.3.2 apresentamos o esquema de Godunov, onde começamos no tempo $t=$ $t_{n}=n \Delta t$ com uma aproximação para a solução, $u_{i}^{n}$. Usamos esse dado para definir uma 
aproximação para a solução, $\bar{u}^{n}$, constante sobre o intervalo $\left(x_{i-1 / 2}, x_{i+1 / 2}\right)$ e $\bar{u}^{n}(x)=u_{i}^{n}$ nesse intevalo. Em seguida, resolvemos o problema de valor inicial consistindo da lei de conservação (3.67) com condição inicial $v\left(x, t_{n}\right)=\bar{u}^{n}$ onde usamos a solução, $\bar{U}=\widehat{U}(x, t)$, no tempo $t=t_{n+1}$ e $x=x_{i}=i \Delta x$, tomando $u_{i}^{n+1}$ igual à solução média de $\bar{U}$ dada por (3.68).

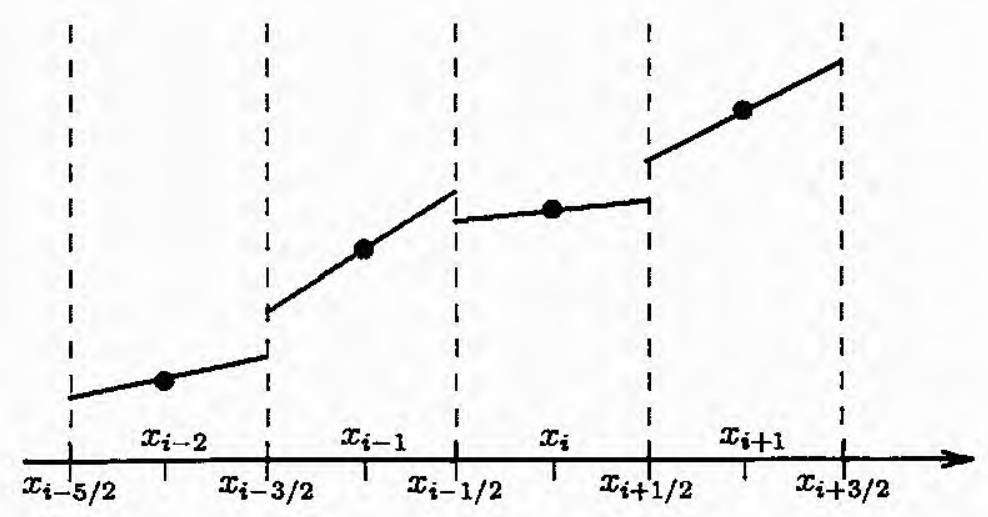

Figura 3.3.15. Exemplo de uma aproximação linear por partes para a solução $\bar{u}^{n}$.

O método limitante de inclinação é muito similar ao método de Godunov. Agora não representaremos a aproximação da solução no tempo $t=t_{n}$ como uma função constante por partes como acima. Usamos o dado $u_{i}^{n}$, no tempo $t=t_{n}$, para definir uma aproximação linear por partes para a solução, $\bar{u}^{n}$, de maneira que $\bar{u}^{n}(i \Delta x)=u_{i}^{n}$ e a inclinação de $\bar{u}^{n}$ no intervalo $\left(x_{i-1 / 2}, x_{i+1 / 2}\right)$ seja dada por $\sigma_{i}^{n}$. A Figura 3.3.15 ilustra a solução aproximada $\bar{u}^{n}$. Portanto, sobre o intervalo $\left(x_{i-1 / 2}, x_{i+1 / 2}\right), \bar{u}^{n}(x)=u_{i}^{n}+\sigma_{i}^{n}\left(x-x_{i}\right)$.

O esquema limitante de inclinação que descrevemos a seguir consiste então em resolver o problema de valor inicial dado por

$$
v_{t}+G_{x}=0, \quad x \in \mathbb{R}, t>0,
$$

$v(x, 0)=\bar{u}^{n}$ para $\bar{U}=\bar{U}(x, t)$. Seja $u_{i}^{n+1}$ a solução média de $\bar{U}$ em $\Delta t$ e defina a solução aproximada $\bar{u}^{n+1}$ sobre a célula $\left(x_{i-1 / 2}, x_{i+1 / 2}\right)$ como $\bar{u}^{n+1}(x)=u_{i}^{n+1}+\sigma_{i}^{n+1}\left(x-x_{i}\right)$. Mais a frente discutiremos como escolher as inclinações $\sigma_{i}^{n}$ e $\sigma_{i}^{n+1}$.

\section{Esquemas Limitante de Inclinaçāo para a Equaçāo de Advecção Linear}

Seja a equação de adveç̧ão linear, $v_{t}+a v_{x}=0$, como podemos resolver esta equação exatamente, veremos que a construção acima produzirá o esquema 


$$
u_{i}^{n+1}= \begin{cases}u_{i}^{n}-a R \delta_{-} u_{i}^{n}-\frac{a R}{2}(1-a R) \Delta x \delta_{-} \sigma_{i}^{n} & \text { se } a>0 \\ u_{i}^{n}-a R \delta_{+} u_{i}^{n}-\frac{a R}{2}(1+a R) \Delta x \delta_{+} \sigma_{i}^{n} & \text { se } a<0 .\end{cases}
$$

Por exemplo, se considerarmos o caso em que $a>0$ (solução propagando à direita) para determinar $\bar{U}$ sobre o intervalo $\left(x_{i-1 / 2}, x_{i+1 / 2}\right)$, devemos considerar a condição inicial sobre ambos os intervalos $\left(x_{i-3 / 2}, x_{i-1 / 2}\right)$ e $\left(x_{i-1 / 2}, x_{i+1 / 2}\right)$. Portanto, devemos resolver o problema consistindo da lei de conservação $v_{t}+a v_{x}=0$ com condição inicial

$$
v\left(x, t_{n}\right)=v_{0}(x)= \begin{cases}u_{i-1}^{n}+\sigma_{i-1}^{n}\left(x-x_{i-1}\right) & \text { para } x_{i-3 / 2} \leq x \leq x_{i-1 / 2} \\ u_{i}^{n}+\sigma_{i}^{n}\left(x-x_{i}\right) & \text { para } x_{i-1 / 2} \leq x \leq x_{i+1 / 2} .\end{cases}
$$

Devido a solução para este problema no tempo $t=t_{n+1}$ pode ser escrita como

$$
v\left(x, t_{n+1}\right)=v_{0}(x-a \Delta t)= \begin{cases}u_{i-1}^{n}+\sigma_{i-1}^{n}\left(x-a \Delta t-x_{i-1}\right), & x_{i-3 / 2} \leq x-a \Delta t \leq x_{i-1 / 2} \\ u_{i}^{n}+\sigma_{i}^{n}\left(x-a \Delta t-x_{i}\right), & x_{i-1 / 2} \leq x-a \Delta t \leq x_{i+1 / 2},\end{cases}
$$

podemos escrever a solução em $u_{i}^{n+1}$ como

$$
\begin{aligned}
u_{i}^{n+1}= & \frac{1}{\Delta x} \int_{x_{i-1 / 2}}^{x_{i+1 / 2}} v_{0}(x-a \Delta t) d x=\frac{1}{\Delta x} \int_{x_{i-1 / 2}}^{x_{i-1 / 2}+a \Delta t}\left[u_{i-1}^{n}+\sigma_{i-1}^{n}\left(x-a \Delta t-x_{i-1}\right)\right] d x \\
& +\frac{1}{\Delta x} \int_{x_{i-1 / 2}+a \Delta t}^{x_{i+1 / 2}}\left[u_{i}^{n}+\sigma_{i}^{n}\left(x-a \Delta t-x_{i}\right)\right] d x .
\end{aligned}
$$

Calculando essa integral, temos a primeira expressão de (3.192). Devemos estar atentos para que seja satisfeita a condição $\mathrm{CFL},|a| R \leq \frac{1}{2}$, que pode ser relaxada para $|a| R \leq 1$ semelhante ao método de Godunov. O esquema (3.192) pode ser escrito em termos da função de fluxo numérico

$$
g_{i+1 / 2}^{n}= \begin{cases}a u_{i}^{n}+\frac{a}{2}(1-a R) \Delta x \sigma_{i}^{n} & \text { se } a>0 \\ a u_{i+1}^{n}-\frac{a}{2}(1+a R) \Delta x \sigma_{i+1}^{n} & \text { se } a<0\end{cases}
$$

e portanto, conservativo. Na forma compacta (3.193) torna-se:

$$
g_{i+1 / 2}^{n}=\frac{1}{2} a\left(u_{i}^{n}+u_{i+1}^{n}\right)-\frac{1}{2}|a| \delta_{+} u_{i}^{n}+\frac{a}{2}(\operatorname{sign}(a)-a R) \Delta x \sigma_{i+l}^{n},
$$

onde $l=0$ se $a>0$ e $l=1$ se $a<0$. Para $a>0$ a escolha

$$
\sigma_{i}^{n}=\frac{\delta_{+} u_{i}^{n}}{\Delta x}
$$

produz o esquema de Lax-Wendroff (3.88). Portanto, é possível escolher a inclinação $\sigma_{i}^{n}$ tal que o esquema seja de segunda ordem e nāo TVD. Para obtermos esquemas TVD, devemos impor limites sobre as inclinaçōes $\sigma_{i}^{n}$, disso deriva o nome métodos limitante de 
inclinação. A seguir enunciamos o seguinte resultado que impõem as condições a serem observadas.

Proposiçāo 3.3.45. Se as inclinações $\sigma_{i}^{n}, i=-\infty, \ldots, \infty$, são escolhidas de maneira que a variação total da solução aproximada linear por partes é menor ou igual a variação total da solução aproximada constante por partes no esquema de Godunov, então o esquema será TVD.

Observação 3.3.46. A hipótese dessa proposição é obviamente muito importante. Observando a Figura 3.3.15, concluimos da expressão (3.198) que a variação entre os pontos $x_{i-2}$ e $x_{i-1}$ é a mesma quando considerado como uma função constante por partes ou como uma função linear por partes. Isto também ocorre quando consideramos os pontos $x_{i}$ e $x_{i+1}$. Entre os pontos $x_{i-1}$ e $x_{i}$, a variação dos dados considerados como uma funçáo linear por partes é maior do que quando considerado como uma função constante por partes. Iremos escolher nossas inclinações $\sigma_{i}^{n}$ de tal maneira que as partes lineares apresentem-se como mostrado em $x_{i-3 / 2}$ e $x_{i+1 / 2}$ e não como elas estão em $x_{i-1 / 2}$. Por exemplo, a variação entre os pontos $x_{i-2}$ e $x_{i-1}$ não é maior do que aquela associada com a função constante por partes, devido ao

$$
\lim _{x \rightarrow x_{i-3 / 2}^{-}} \bar{u}^{n}(x) \leq \lim _{x \rightarrow x_{i-3 / 2}^{+}} \bar{u}^{n}(x) .
$$

Igualmente, a variação de $\bar{u}^{n}$ entre os pontos $x_{i-1}$ e $x_{i}$ será maior do que aquela associada com a função constante por partes devido ao

$$
\lim _{x \rightarrow x_{i-1 / 2}^{-}} \bar{u}^{n}(x)>\lim _{x \rightarrow x_{i-1 / 2}^{+}} \bar{u}^{n}(x) .
$$

Assim vimos que devemos escolher cuidadosamente as inclinações para que o esquema tenha a propriedade TVD. Um simples limitante de inclinação é dado pela inclinação minmod,

$$
\sigma_{i}^{n}=\frac{1}{\Delta x} \min \bmod \left\{\delta_{+} u_{i}^{n}: \delta_{-} u_{i}^{n}\right\}
$$

onde a função minmod é definida por

$$
\min \bmod \{a, b\}=\frac{1}{2}(\operatorname{sign}(a)+\operatorname{sign}(b)) \min (|a|,|b|)= \begin{cases}a & \text { se }|a|<|b| \text { e } a b>0 \\ b & \text { se }|b|<|a| \text { e } a b>0 \\ 0 & \text { se } a b \leq 0\end{cases}
$$


Veremos através de um exemplo que as escolhas das inclinações utilizando a função minmod satisfaz a Proposição 3.3.45. Assim, para uma função linear por partes $\bar{u}(x)=$ $u_{i}^{n}+\sigma_{i}^{n}\left(x-x_{i}\right), x \in\left(x_{i-1 / 2}, x_{i+1 / 2}\right)$, dcfinimos a variação linear total por:

$$
T V\left(\bar{u}_{\left.\right|_{\left(x_{i-1}, x_{i}\right)}}\right)=\left|u_{i}^{n}-u_{i-1 / 2^{+}}^{n}\right|+\left|u_{i-1 / 2^{+}}^{n}-u_{i-1 / 2^{-}}^{n}\right|+\left|u_{i-1 / 2^{-}}^{n}-u_{i-1}^{n}\right| .
$$

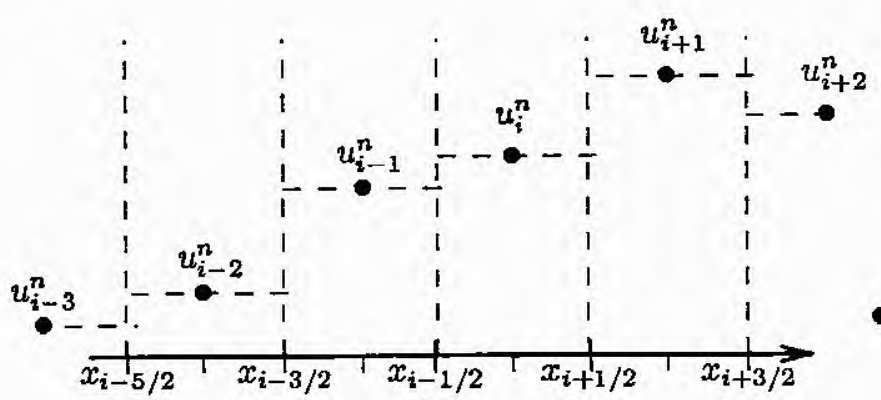

(a)

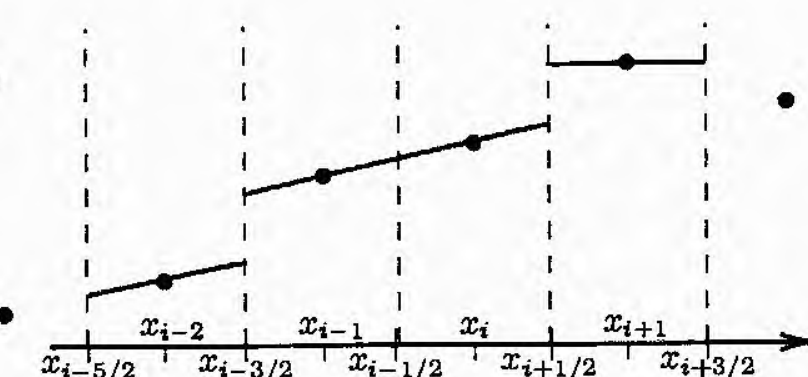

(b)

A figura (a) mostra os valores da solução no tempo $t=t_{n}$. Primeiramente, determinamos as inclinações da função linear que passam por esses valores, conforme mostrado em (b). A seguir, comparamos a variação total de Godunov (constante) com a variação total determinada pela função minmod (linear) e para medir essa última variação, fizemos uso da expressão (3.198).

Para determinar a inclinação em $u_{i}^{n}$ precisamos dos valores $u_{i-1}^{n}$ e $u_{i+1}^{n}$. Através da figura observamos que $\delta_{+} u_{i}^{n}>\delta_{-} u_{i}^{n}$ e ambas as diferenças são positivas. Pela definição da função minmod temos que $\sigma_{i}^{n}=\frac{\delta_{-} u_{i}^{n}}{\Delta x}$. Da mesma forma, para determinar a inclinação em $u_{i-1}^{n}$ necessitamos dos valores $u_{i-2}^{n}$ e $u_{i}^{n}$. Assim temos que $\delta_{+} u_{i-1}^{n}<\delta_{-} u_{i-1}^{n}$ com ambas variações positivas e portanto $\sigma_{i-1}^{n}=\frac{\delta_{+} u_{i-1}^{n}}{\Delta x}=\sigma_{i}^{n}$ conforme mostrado em (b). Tendo ambas inclinações, temos que $T V\left(\bar{u}_{\left.\right|_{\left(x_{i-1}, x_{i}\right)}}\right)=\left|u_{i}^{n}-u_{i-1 / 2^{+}}^{n}\right|+0+\left|u_{i-1 / 2^{+}}^{n}-u_{i-1}^{n}\right|=\left(u_{i}^{n}-u_{i-1}^{n}\right)=$ variação da solução obtida pelo método de Godunov, que é o que a Proposição 3.3.45 afirma. A determinação das inclinações para os demais valores bem como as variações para a verificação com a proposição são feitas de maneiras análogas.

As inclinações $\sigma_{i}^{n}$ podem ser escolhidas de tal maneira que os métodos limitantes de fluxo possam ser considerados como casos especiais dos esquemas limitantes de inclinação. Por exemplo, se escolhermos as inclinações $\sigma_{i}^{n}$ como $\sigma_{i}^{n}=\phi_{i}^{n} \frac{u_{i+1}^{n}-u_{i}^{n}}{\Delta x}$, então o esquema limitante de inclinação (3.192) é igual ao esquema limitante de fluxo associado com a função de fluxo numérico (3.181). Esta breve introdução a métodos limitantes de inclinação para equações lineares facilitará nossa abordagem para a derivação de métodos não lineares. 


\section{Esquemas Limitante de Inclinação para Leis de Conservação não Lineares}

A diferença entre os casos linear e não linear é que não é possível resolver exatamente o problema local que consiste da lei de conservação (3.191) com condição inicial $v\left(x, t_{n}\right)=$ $\bar{u}^{n}(x)=u_{i}^{n}+\sigma_{i}^{n}\left(x-x_{i}\right)$. Se voltarmos à seção 3.3 .2 , notamos que a equação (3.71) não depende da forma de $\bar{u}^{n}$. De fato, devido à definição de $\bar{u}^{n}$, a equação (3.72) vai sempre ser satisfeita. Gostaríamos de definir as funções de fluxo numérico como fizemos antes na equação (3.73) como

$$
g_{i+1 / 2}^{n}=\frac{1}{\Delta t} \int_{t_{n}}^{t_{n+1}} G\left(\bar{U}\left(x_{i+1 / 2}, t\right)\right) d t
$$

onde $\bar{U}$ é agora a solução da lei de conservação (3.191) com condição inicial $v\left(x, t_{n}\right)=\bar{u}^{n}(x)=$ $u_{i}^{n}+\sigma_{i}^{n}\left(x-x_{i}\right)$. Esta função de fluxo numérico deverá ser uma extensão natural do método de Godunov. Entretanto, nós não sabemos calcular $g_{i \pm 1 / 2}^{n}$, assim como não sabemos calcular $\bar{U}$ para uma função linear por partes. A técnica descrita em [17], é usar uma aproximação de $G$ para calcular uma aproximação de $\bar{U}$ e por conseguinte definirmos uma aproximação para $g_{i+1 / 2}^{n}$. Como a descrição do algoritmo torna-se mais complicada próximo dos pontos críticos da solução e de pontos sônicos, assumimos no momento que a solução não possui pontos sônicos na região que estamos considerando, isto é, $G^{\prime}\left(v\left(x, t_{n}\right)\right) \neq 0$ e que $G$ é sempre convexa.

Começamos definindo $\sigma_{i}^{n}$ como fizemos antes

$$
\sigma_{i}^{n}=\frac{1}{\Delta x} \min \bmod \left\{\delta_{+} u_{i}^{n}, \delta_{-} u_{i}^{n}\right\}
$$

Esta escolha de $\sigma_{i}^{n}$ é feita de tal maneira que a variação de $\bar{u}^{n}$ é a mesma de $u_{i}^{n}$. Definimos:

$$
U_{i}^{ \pm}=u_{i}^{n} \pm \frac{1}{2} \Delta x \sigma_{i}^{n}
$$

Notamos que a variação total da aproximação linear por partes da solução é menor ou igual à variação pontual da solução aproximada e por virtude da escolha das inclinações (3.197), os pontos $U_{i}^{-}, U_{i}^{+}, U_{i+1}^{-}, U_{i+1}^{+}$, estão monotonicamente ordenados (embora dois ou mais possam coincidir), ie, $U_{i}^{-} \leq U_{i}^{+} \leq U_{i+1}^{-} \leq U_{i+1}^{+}$ou $U_{i}^{-} \geq U_{i}^{+} \geq U_{i+1}^{-} \geq U_{i+1}^{+}$. Na Figura 3.3.16, plotamos $\bar{u}^{n}$ e $U_{j}^{ \pm}, j=i, i+1$ para vários valores de $u_{i}^{n}$. 


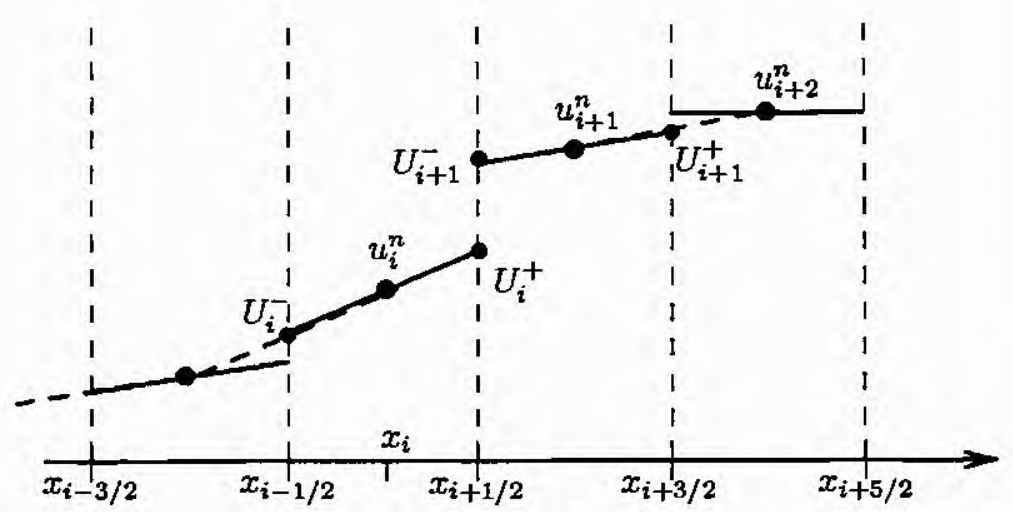

Figura 3.3.16. Exemplo de uma porção da solução aproximada $\bar{u}^{n}$. As linhas tracejadas mostram como as inclinações $\sigma_{i}^{n}$ são determinadas. Os pontos $U_{j}^{ \pm}$são plotados para $j=i \mathrm{e}$ $j=i+1$.

Seja $F=F(u)$ a função linear por partes que interpola $G$ nos quatro pontos $U_{i}^{ \pm}$e $U_{i+1}^{ \pm}$ e defina

$$
F_{i}^{\prime}= \begin{cases}{\left[G\left(U_{i}^{+}\right)-G\left(U_{i}^{-}\right)\right] /\left(U_{i}^{+}-U_{i}^{-}\right)} & \text {se } \sigma_{i}^{n} \neq 0 \\ G^{\prime}\left(u_{i}^{n}\right) & \text { se } \sigma_{i}^{n}=0 .\end{cases}
$$

Então $F_{i}^{\prime}$ é a inclinação de $F$ entre $U_{i}^{-}$e $U_{i}^{+}$, portanto, a função $F$ é dada por

$$
F(u)= \begin{cases}G\left(U_{i}^{+}\right)+\left(u-U_{i}^{+}\right) G_{i}^{\prime} & \text { para } u \in\left[U_{i}^{-}, U_{i}^{+}\right] \\ G\left(U_{i+1}^{-}\right)+\left(u-U_{i+1}^{-}\right) G_{i+1}^{\prime} & \text { para } u \in\left[U_{i+1}^{-}, U_{i+1}^{+}\right] .\end{cases}
$$

Um exemplo de uma função de fluxo aproximante $F$ é ilustrado na Figura 3.3.17. Notamos que ela interpola os pontos $U_{j}^{ \pm}$para $j=i$ e $j=i+1$. Nessa figura vemos que as porções lineares se interceptam em um valor $U=U_{i}^{*}$.

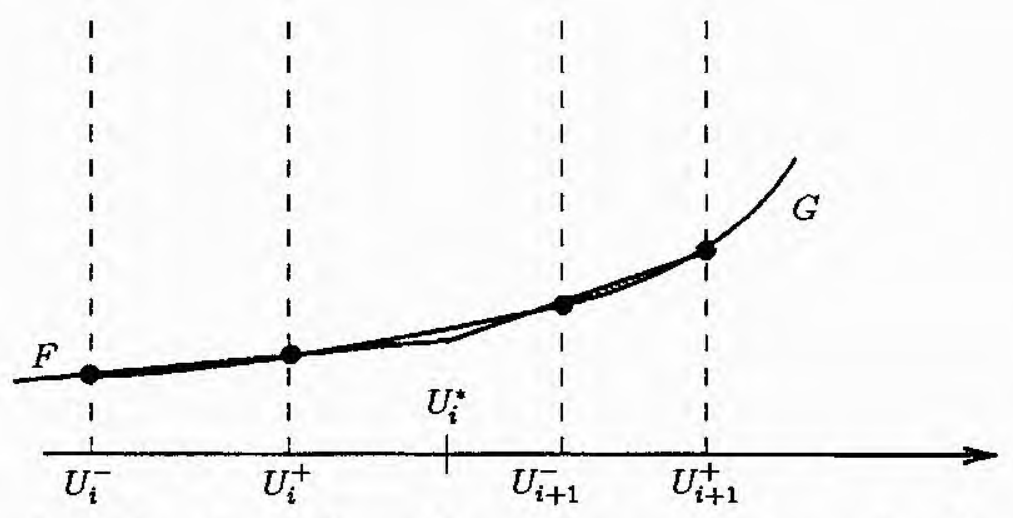

Figura 3.3.17. Ilustração da função de fluxo $F$ que interpola linearmente a função de fluxo $G$ nos pontos $U_{j}^{ \pm}, j=i$ e $j=i+1$.

A seguir, substituimos a função $G$ em (3.199) pela função $F$ e consideramos o problema de valor inicial: 


$$
\begin{aligned}
& v_{t}+F_{x}=0 \\
& v\left(x, t_{n}\right)=\bar{u}^{n} .
\end{aligned}
$$

Se escrevermos a equação (3.204) na forma não conservativa, temos $v_{t}+F^{\prime} v_{x}=0$. Salientamos que $F$ não é diferenciável em toda parte, e assim devemos tomar alguns cuidados. $O$ importante a ser considerado é que por ser $F$ linear por partes, temos que $F^{\prime}$ é uma constante e portanto podemos resolver a equação. Precisamos calcular a solução para o problema (3.204)-(3.205) em $x=x_{i+1 / 2}$. Começamos considerando o caso em que $G^{\prime}>0$. Quando $G^{\prime}>0$, queremos também que $F^{\prime}>0$. Isto envolverá escolher $\Delta x$ suficientemente pequeno tal que $F$ seja uma boa aproximação para $G$. Se $G^{\prime}>0$, a solução vai se propagar à direita e neste caso a solução em $x=x_{i+1 / 2}$ é determinada por $\bar{u}, G$ e $F^{\prime}$ no intervalo $\left(x_{i-1 / 2}, x_{i+1 / 2}\right)$. A condição inicial relevante será $v_{0}(x)=u_{i}^{n}+\sigma_{i}^{n}\left(x-x_{i}\right)$, a solução exata será $v(x, t)=v_{0}\left(x-F_{i}^{\prime}\left(t-t_{n}\right)\right)$, e $\bar{U}$ é então dada por:

$$
\begin{aligned}
\bar{U}\left(x_{i+1 / 2}, t\right) & =v_{0}\left(x_{i+1 / 2}-F^{\prime}{ }_{i}\left(t-t_{n}\right)\right) \\
& =u_{i}^{n}+\sigma_{i}^{n}\left(x_{i+1 / 2}-F_{i}^{\prime}\left(t-t_{n}\right)-x_{i}\right) \\
& =U_{i}^{+}-F_{i}^{\prime} \sigma_{i}^{n}\left(t-t_{n}\right) .
\end{aligned}
$$

Se $G^{\prime}<0$, o resultado será semelhante mas dependerá da solução no itervalo $\left(x_{i+1 / 2}, x_{i+3 / 2}\right)$ e de $F_{i+1}^{\prime}$. Portanto, a solução $\bar{U}=\bar{U}(x, t)$ em $x=x_{i+1 / 2}$ para o problema de valor inicial (3.204)-(3.205) é

$$
\bar{U}\left(x_{i+1 / 2}, t\right)= \begin{cases}U_{i}^{+}-\left(t-t_{n}\right) \sigma_{i}^{n} F_{i}^{\prime} & \text { se } G^{\prime}>0 \\ U_{i+1}^{-}-\left(t-t_{n}\right) \sigma_{i+1}^{n} F_{i+1}^{\prime} & \text { se } G^{\prime}<0\end{cases}
$$

A função de fluxo numérico $\tilde{g}_{i+1 / 2}^{n}$ vai se aproximar da função de fluxo numérico (3.199) e será

$$
\tilde{g}_{i+1 / 2}^{n}=\frac{1}{\Delta t} \int_{t_{n}}^{t_{n+1}} F\left(\bar{U}\left(x_{i+1 / 2}, t\right)\right) d t .
$$

Usando $F$ dada em (3.203), e depois $(3.207)$, vemos que $F\left(\bar{U}\left(x_{i+1 / 2}, t\right)\right)$ é dada por (novamente para o caso quando $G^{\prime}>0$ ) 


$$
\begin{aligned}
F\left(\bar{U}\left(x_{i+1 / 2}, t\right)\right) & =G\left(U_{i}^{+}\right)+\left(\bar{U}\left(x_{i+1 / 2}, t\right)-U_{i}^{+}\right) F_{i}^{\prime} \\
& =G\left(U_{i}^{+}\right)-\left(t-t_{n}\right) \sigma_{i}^{n}\left(F_{i}^{\prime}\right)^{2} .
\end{aligned}
$$

Para $G^{\prime}<0$ os resultados são muito similares. Então, usando (3.209) em (3.208) quando $G^{\prime}>0$ e o resultado análogo para quando $G^{\prime}<0$, temos

$$
\begin{aligned}
\tilde{g}_{i+1 / 2}^{n} & =\frac{1}{\Delta t} \int_{t_{n}}^{t_{n+1}} F\left(\bar{U}\left(x_{i+1 / 2}, t\right)\right) d t \\
& = \begin{cases}G\left(U_{i}^{+}\right)-\frac{1}{2} \Delta t \sigma_{i}^{n}\left(F_{i}^{\prime}\right)^{2} & \text { se } G^{\prime}>0 \\
G\left(U_{i+1}^{-}\right)-\frac{1}{2} \Delta t \sigma_{i+1}^{n}\left(F_{i+1}^{\prime}\right)^{2} & \text { se } G^{\prime}<0\end{cases}
\end{aligned}
$$

Se existe um ponto sônico na região, isto é, se $F_{i}^{\prime} F_{i+1}^{\prime} \leq 0$, então definimos

$$
F_{i+1 / 2}^{\prime}= \begin{cases}{\left[G\left(U_{i+1}^{-}\right)-G\left(U_{i}^{+}\right)\right] /\left[U_{i+1}^{-}-U_{i}^{+}\right]} & \text {se } U_{i+1}^{-} \neq U_{i}^{+} \\ G^{\prime}\left(U_{i}^{+}\right) & \text {se } U_{i+1}^{-}=U_{i}^{+}\end{cases}
$$

e

(1) se $F_{i}^{\prime}>0, F_{i+1 / 2}^{\prime}\left(U_{i+1}^{-}-U_{i}^{+}\right)=0$ e $F_{i+1}^{\prime}<0$, então

$$
\tilde{g}_{i+1 / 2}^{n}= \begin{cases}G\left(U_{i}^{+}\right)-\frac{1}{2} \Delta t \sigma_{i}^{n}\left(F_{i}^{\prime}\right)^{2} & \text { se } \sigma_{i}\left(F_{i}^{\prime}\right)^{2} \geq \sigma_{i+1}^{n}\left(F_{i+1}^{\prime}\right)^{2} \\ G\left(U_{i+1}^{-}\right)-\frac{1}{2} \Delta t \sigma_{i+1}^{n}\left(F_{i+1}^{\prime}\right)^{2} & \text { caso contrário }\end{cases}
$$

(2) caso contrário, defina

$$
\tilde{g}_{i+1 / 2}^{n}= \begin{cases}G\left(U_{i}^{+}\right)-\frac{1}{2} \Delta t \sigma_{i}^{n}\left(F_{i}^{\prime}\right)^{2} & \text { se } F_{i}^{\prime} \geq 0 \text { e } F_{i+1 / 2}^{\prime} \geq 0 \\ G\left(U_{i+1}^{-}\right)-\frac{1}{2} \Delta t \sigma_{i+1}^{n}\left(F_{i+1}^{\prime}\right)^{2} & \text { se } F_{i+1}^{\prime} \leq 0 \text { e } F_{i+1 / 2}^{\prime} \leq 0 \\ G\left(v_{0}\right) & \text { se } F_{i}^{\prime}<0 \text { e } F_{i+1}^{\prime}>0\end{cases}
$$

onde $v_{0}=\min \left\{\max \left\{U_{i}^{+}, u_{S}\right\}, U_{i+1}^{-}\right\}$e $u_{S}$ é o ponto sônico. Para mais detalhes envolvendo estes desenvolvimentos veja [17].

Se portanto tivermos $F_{i}^{\prime} F_{i+1}^{\prime}>0$, defina $\tilde{g}$ pela equação (3.211). Caso contrário, use as expressões (3.212), (3.213) e (3.214). A proposição seguinte fornece um dos resultados mais importante dos esquemas limitantes de inclinação desenvolvidos acima.

Proposição 3.3.47. Se $R\left|G^{\prime}\right| \leq \frac{1}{2}$, o esquema de diferenças definido pela função de fluxo numérico (3.210), (3.212)-(3.214) é de segunda ordem de precisão sobre seções suaves da solução que não sejam pontos críticos. 
Consideremos o caso em que não temos um ponto crítico local da solução $\left(\sigma_{i}^{n} \neq 0\right)$ e discutamos a prova para $G^{\prime}>0$ (o caso $G^{\prime}<0$ é similar). Podemos ver que a função de fluxo numérico (3.211) é similar à função de fluxo numérico para o esquema de Lax-Wendroff (3.93) que é dada por

$$
g_{i+1 / 2}^{\mathrm{LW}}=\frac{1}{2}\left(G_{i}^{n}+G_{i+1}^{n}\right)-\frac{R}{2}\left(a_{i+1 / 2}^{n}\right)^{2} \delta_{+} u_{i}^{n}
$$

Se expandirmos a expressão $\tilde{g}_{i+1 / 2}^{n}-g_{i+1 / 2}^{\mathrm{LW}}$ em relação a $u_{i}^{n}$, fornece

$$
\tilde{g}_{i+1 / 2}^{n}-g_{i+1 / 2}^{\mathrm{LW}}=\frac{1}{2}\left[R G^{\prime}\left(u_{i}^{n}\right)-1\right]\left[\delta_{+} u_{i}^{n}-\Delta x \sigma_{i}^{n}\right]+o(\Delta x)^{2} .
$$

Para $\sigma_{i}^{n}=\delta_{+} u_{i}^{n} / \Delta x$, o primeiro termo depois da igualdade da equação acima é zero. Para $\sigma_{i}^{n}=\delta_{-} u_{i}^{n} / \Delta x$, este termo passa a ter ordem $\Delta x^{2}$. Portanto, em ambos os casos temos que $\tilde{g}_{i+1 / 2}^{n}-g_{i+1 / 2}^{\mathbf{L w}}=o\left(\Delta x^{2}\right)$, e pela Proposição 3.3 .32 , o esquema limitante de inclinação é de segunda ordem de precisão.

Para mais detalhes sobre métodos limitantes de inclinação sugerimos as referências [15] e [17].

Usando alguns esquemas desenvolvidos nessa seção para uma lei de conservação, construiremos no Capítulo 4 esquemas de primeira e segunda ordem para os problemas de valor inicial e de fronteira desenvolvidos no Capítulo 1; incluiremos também algoritmos para Hamiltonianos multidimensionais e não-convexos. 


\section{Capítulo 4}

\section{Esquemas Hiperbólicos e Equações de Hamilton-Jacobi}

\subsection{Introdução}

Neste capítulo, estudamos esquemas numéricos especialmente projetados para resolver as formulações,

formulaçāo de valor de fronteira

$$
|\nabla T| F=1
$$

Frente $=\Gamma(t)=\{(x, y): T(x, y)=t\}$ restrição $F>0$ formulaçāo de valor inicial

$$
\phi_{t}+F|\nabla \phi|=0
$$

$$
\text { Frente } \begin{gathered}
=\Gamma(t)=\{(x, y): \phi(x, y, t)=0\} \\
F \text { geral }
\end{gathered}
$$

para o movimento de interfaces. O fato a ser destacado é que esses esquemas são derivados de um elo importante entre as equações de Hamilton-Jacobi e as leis de conservação hiperbólicas. Apesar dessa analogia ser equivalente somente para equações unidimensionais, a metodologia pode ser estendida para esquemas multidimensionais para a propagação de interfaces.

No que se segue, construiremos esquemas numéricos para as equações de Hamilton-Jacobi que convergem para a solução de viscosidade. Crandall e Lions [9] provaram que esquemas consistentes monótonos convergem para a solução de viscosidade; este resultado é análogo para leis de conservação hiperbólicas unidimensionais. Assim, podemos simplesmente derivar esquemas baseados em (3.85) e então checar se eles satisfazem as exigências necessárias para convergência para a solução de viscosidade. Entretanto, melhor do que derivar esquemas e provar que eles satisfazem as exigências para convergência, estamos interessados em derivar esquemas para as equaçōes de Hamilton-Jacobi baseados no que foi feito para as leis de conservação hiperbólicas. Assim sendo, tais esquemas foram derivados utilizando propriedades 
de esquemas Upwind, Monótonos e TVD, devido à simplicidade dos mesmos, são naturalmente compreendidos no que concerne à direção de propagação e características, e porque fornecem a solução entrópica.

Salientamos que existem esquemas mais sofisticados do que os apresentados aqui. Nas aplicações desses esquemas a problemas hiperbólicos, esquemas de alta resolução são frequentemente usados, devido a diferenciação da função de fluxo numérico $g$ contribuir no aumento de difusividade (smearing) na solução por parte dos esquemas. Entretanto, em nosso caso, como iremos resolver $\phi_{t}+H(u)=0 \mathrm{em}$ vez de $u_{t}+[H(u)]_{x}=0$, a diferenciação não é exigida. A seguir, apresentamos as principais referências utilizadas, as quais são: [29], [30],[38], [40], encontradas na bibliografia.

\subsection{Esquemas Unidimensionais}

Ambas as formulações para o problema de propagação de interfaces podem ser escritas na forma geral da equação de Hamilton-Jacobi

$$
\alpha U_{t}+H\left(U_{x_{1}}, U_{x_{2}}, \ldots, U_{x_{N}}, x_{1}, x_{2}, \ldots, x_{N}\right)=0
$$

em que a função $H$ é conhecida como o Hamiltoniano.

Para o problema de valor inicial

$$
H\left(U_{x_{1}}, U_{x_{2}}, \ldots, U_{x_{N}}, x_{1}, x_{2}, \ldots, x_{N}\right)=F\left(x_{1}, x_{2}, \ldots, x_{N}\right) \sqrt{U_{x_{1}}^{2}+U_{x_{2}}^{2}+\ldots, U_{x_{N}}^{2}},
$$

e para o problema de valor de fronteira

$$
H\left(U_{x_{1}}, U_{x_{2}}, \ldots, U_{x_{N}}, x_{1}, x_{2}, \ldots, x_{N}\right)=F\left(x_{1}, x_{2}, \ldots, x_{N}\right) \sqrt{U_{x_{1}}^{2}+U_{x_{2}}^{2}+\ldots, U_{x_{N}}^{2}}-1 .
$$

com $\alpha=1$ e $\alpha=0$, respectivamente.

Observação 4.2.1. Os métodos de Conjuntos de Níveis são técnicas computacionais baseadas na solução de uma equação do tipo Hamilton-Jacobi para uma função de propagação (conjunto de nível) usando técnicas herdadas de leis de conservação hiperbólicas. Analogamente, os métodos de marcha rápida são técnicas computacionais que aproximam a solução para as equações não-lineares, chamadas equações Eikonal, obtidas tomando o Hamiltoniano do problema de valor de fronteira igual a zero, ou seja,

$$
|\nabla u(x)|=\mathcal{F}(x) \text { em } \Omega, \quad \mathcal{F}(x)>0,
$$




$$
u=h(x) \text { sobre } \Gamma
$$

onde $\Omega$ é um domínio em $\mathbb{R}^{n}, \quad \mathcal{F}(x)=1 / F(x)$ e h é uma função conhecida ao longo de uma curva ou superfície em $\Omega$. Essa equação faz parte de uma ampla classe das equações de Hamilton-Jacobi da forma

$$
H\left(U_{x_{1}}, U_{x_{2}}, \ldots, U_{x_{N}}, x_{1}, x_{2}, \ldots, x_{N}\right)=0
$$

No caso da equação Eikonal, a função $H$ reduz-se a $H=|\nabla u(x)|-\mathcal{F}(x)$.

A equação de Hamilton-Jacobi para o caso unidimensional $(N=1)$ é:

$$
\alpha U_{t}+H\left(U_{x_{1}}\right)=0
$$

Se fizermos $x_{1}=x$ e $U_{x}=u$ e diferenciarmos (4.4) em relação à variável espacial temos a lei de conservação hiperbólica para $\alpha=1$

$$
u_{t}+[H(u)]_{x}=0
$$

Observação 4.2.2. Estamos usando a variável u para denotar a solução da lei de conservação hiperbólica, e U para denotar a solução da equação de Hamilton-Jacobi.

Mostraremos agora, através de um exemplo, a transformação de uma equação unidimensional de Hamilton-Jacobi para uma lei de conservação hiperbólica, conforme descrevemos acima.

Exemplo 4.2.3. Considere

$$
\begin{aligned}
U_{t}+H\left(U_{x}\right) & =0 \\
U(x, 0) & =-\cos (\pi x),-1 \leq x \leq 1 .
\end{aligned}
$$

Diferenciando a equação diferencial (4.6) em relação a variável $x$ e fazendo a mudança de variável $U_{x}=u$, e também chamando $H(u)=G(u)$, temos

$$
u_{t}+G(u)_{x}=0
$$

A condição inicial (4.7) é transformada da seguinte maneira: 
$u(x, t)=U_{x}(x, t), \forall(x, t) \in \operatorname{dom} U \rightarrow u(x, 0)=U_{x}(x, 0) \rightarrow \int u(x, 0) d x=\int U_{x}(x, 0) d x+c$ $=U(x, 0)+k=-\cos (\pi x)+k$. Portanto temos que $\int u(x, 0) d x=-\cos (\pi x)+k$. Diferenciando essa expressão em $x$ obtêm-se $u(x, 0)=\operatorname{sen}(\pi x) \pi$.

$$
\begin{aligned}
u_{t}+G(u)_{x} & =0 \\
u(x, 0) & =\operatorname{sen}(\pi x) \pi,-1 \leq x \leq 1 .
\end{aligned}
$$

Como vimos no capítulo anterior, a função de fluxo numérico $g$ para aproximar (4.5) pode ser construída através da expressão:

$$
\frac{u_{i}^{n+1}-u_{i}^{n}}{\Delta t}=-\frac{g\left(u_{i}^{n}, u_{i+1}^{n}\right)-g\left(u_{i-1}^{n}, u_{i}^{n}\right)}{\Delta x}
$$

Analogamente ao que fizemos para a função de fluxo $G$, os valores de $H$ nos pontos ( $i \pm$ $1 / 2) \Delta x$, denotados por $H_{i \pm 1 / 2}$, serão aproximados pela função de fluxo numérico $g$, como

$$
\begin{aligned}
& H_{i+1 / 2} \approx g\left(u_{i}^{n}, u_{i+1}^{n}\right) \\
& H_{i-1 / 2} \approx g\left(u_{i-1}^{n}, u_{i}^{n}\right) .
\end{aligned}
$$

Como anteriormente, quando o tamanho da malha tende a zero, a condição de consistência é $g(u, u)=H(u)$.

Isto é suficiente para construir um esquema unidimensional para a equação de HamiltonJacobi. Tomando $u=U_{x}$, podemos escrever

$$
U_{t}+H(u)=0
$$

Usando a aproximação $H\left(u_{i}^{n}\right) \approx g\left(u_{i-1 / 2}, u_{i+1 / 2}\right)$, a condição de consistência e aproximando $U_{x}$ por diferenças regressivas e progressivas, temos o esquema para a equação unidimensional (4.12),

$$
U_{i}^{n+1}=U_{i}^{n}-\Delta \operatorname{tg}\left(\frac{U_{i}^{n}-U_{i-1}^{n}}{\Delta x}, \frac{U_{i+1}^{n}-U_{i}^{n}}{\Delta x}\right)
$$

em que $g$ é a função de fluxo numérico. 


\subsection{Esquemas Numéricos em Dimensões Maiores}

Analogamente ao caso unidimensional, a construção de esquemas multidimensionais para as equações de Hamilton-Jacobi, no caso de Hamiltonianos simétricos, pode ser feita simplesmente repetindo o processo anterior para cada uma das variáveis. Por exemplo, para a equação homogênea

$$
U_{t}+H\left(U_{x}, U_{y}, U_{z}\right)=0
$$

pondo $U_{x}=u, U_{y}=v, U_{z}=w$ temos que a condição de consistência é $g(u, u, v, v, w, w)=$ $H(u, v, w)$. A equação (4.14) pode ser aproximada por

$$
\begin{array}{r}
U_{i, j, k}^{n+1}=U_{i, j, k}^{n}-\Delta \operatorname{tg}\left(\frac{U_{i, j, k}^{n}-U_{i-1, j, k}^{n}}{\Delta x}, \frac{U_{i+1, j, k}^{n}-U_{i, j, k}^{n}}{\Delta x},\right. \\
\left.\frac{U_{i, j, k}^{n}-U_{i, j-1, k}^{n}}{\Delta y}, \frac{U_{i, j+1, k}^{n}-U_{i, j, k}^{n}}{\Delta y}, \frac{U_{i, j, k}^{n}-U_{i, j, k-1}^{n}}{\Delta z}, \frac{U_{i, j, k+1}^{n}-U_{i, j, k}^{n}}{\Delta z}\right) .
\end{array}
$$

Uma versão multidimensional desse mesmo esquema em função do fluxo numérico $g^{\text {Hs }}$, é dada por

$$
\begin{gathered}
g^{\mathrm{H} 3}\left(u_{1}, u_{2}, v_{1}, v_{2}, w_{1}, w_{2}\right)=\left[\max \left(u_{1}, 0\right)^{2}+\min \left(u_{2}, 0\right)^{2}+\right. \\
\left.\max \left(v_{1}, 0\right)^{2}+\min \left(v_{2}, 0\right)^{2}+\max \left(w_{1}, 0\right)^{2} \min \left(w_{2}, 0\right)^{2}\right]^{1 / 2}
\end{gathered}
$$

\subsection{O Problema de Valor Inicial: Método dos Conjun- tos de Níveis}

A equação de Hamilton-Jacobi (H-J)

$$
\phi_{t}+H\left(\phi_{x_{1}}, \ldots, \phi_{x_{N}}\right)=0, \quad \phi(x, 0)=\phi_{0}(x)
$$

aparece em várias aplicações como já mencionamos. As soluções de (4.17) são tipicamente contínuas, mas com derivadas descontínuas, mesmo se a condição inicial $\phi(x, 0)$ é $C^{\infty}$. Estendendo o conceito de solução para incluir as soluções não diferenciáveis em todo o domínio, isto é, admitindo-se a possibilidade de soluções fracas, temos que essas soluções não são únicas e portanto é necessário a introdução das noções de condição de entropia e soluçōes de viscosidade para garantir uma única solução relevante para o problema físico em questão. 
Existe uma relação entre (4.17) e uma lei de conservação hiperbólica na forma

$$
u_{t}+\sum_{i=1}^{d} G_{i}(u)_{x_{i}}=0, \quad u(x, 0)=u_{0}(x) .
$$

De fato, conforme vimos anteriormente, para o caso unidimensional $N=1$, (4.17) é equivalente a (4.18) se tomarmos $u=\phi_{x}$. Para dimensões mais altas, esta correspondência direta desaparece, mas espera-se que podemos ainda pensar que (4.17) pode ser derivado de (4.18) "integrado uma vez". Portanto a metodologia numérica utilizada para resolver leis de conservação hiperbólicas como (4.18) deverá ser aplicável à equação H-J (4.17) com sucesso.

$\mathrm{Na}$ sequência apresentamos um novo esquema upwind monótono citado em [29] para o caso particular em que $H(u)=f\left(u^{2}\right)$. No caso particular do problema de valor inicial dado pela equação da onda não linear

$$
u_{t}+\left[u^{2}\right]_{x}=0
$$

seguindo a referência [29] definimos:

$$
g^{\mathrm{HJ}}\left(u_{i}^{n}, u_{i+1}^{n}\right)=f\left(\max \left(u_{i+1}^{n}, 0\right)^{2}+\min \left(u_{i}^{n}, 0\right)^{2}\right), \text { se } f^{\prime}(u)<0 \text {. }
$$

Para o caso unidimensional, por exemplo, $H(u)=f\left(u^{2}\right)=F(x) \sqrt{u^{2}}$, temos que $f^{\prime}(u)=$ $\frac{F(x)}{2 \sqrt{u}}<0$ se e só se $F(x)<0$. Se $f^{\prime}(u)>0$ temos que $F(x)>0$ e definimos:

$$
g^{\mathrm{HJ}}\left(u_{i}^{n}, u_{i+1}^{n}\right)=f\left(\max \left(u_{i}^{n}, 0\right)^{2}+\min \left(u_{i+1}^{n}, 0\right)^{2}\right) .
$$

Assim, definimos o esquema em função da velocidade $F$ por:

$$
g^{\mathrm{HJ}}\left(u_{i}^{n}, u_{i+1}^{n}\right)= \begin{cases}f\left(\max \left(u_{i}^{n}, 0\right)^{2}+\min \left(u_{i+1}^{n}, 0\right)^{2}\right), & \text { se } F(x)>0 \\ f\left(\max \left(u_{i+1}^{n}, 0\right)^{2}+\min \left(u_{i}^{n}, 0\right)^{2}\right), & \text { se } F(x)<0 .\end{cases}
$$

Podemos por exemplo aplicar o esquema (4.22), com $F=1$, para o PVI (4.17) com $N=1$ e $H(u)=\sqrt{u^{2}}$, obtendo

$$
\phi_{i}^{n+1}=\phi_{i}^{n}-\Delta t\left(\max \left(D^{-x} \phi_{i}^{n}, 0\right)^{2}+\min \left(D^{+x} \phi_{i}^{n}, 0\right)^{2}\right)^{1 / 2}
$$

Resultado de uma Aproximação Upwind para a Equação Conjunto de Nível 
Para ilustrar essas idéias vamos utilizar novamente o exemplo da propagação do gráfico de uma função de $\mathbb{R}$ em $\mathbb{R}$. Na Seçāo 2.4 , uma aproximação por diferenças centrais foi usada para rastrear a propagação de um "V" com velocidade $F=1$. A equação de movimento para a função altura $\psi$ foi dada na Equação (2.16) por

$$
\psi_{t}=F\left(1+\psi_{x}^{2}\right)^{1 / 2}
$$

A discretização por diferenças centrais falhou (veja Figuras 2.4.4) pois não tratou a singularidade no canto corretamente. Munido com esse conhecimento, faremos uso de um esquema upwind desenvolvido nessa seção. Neste caso, o Hamitoniano é

$$
H\left(\psi_{x}\right)=-F\left(1+\psi_{x}^{2}\right)^{1 / 2}
$$

e assim simplesmente aplicamos o esquema (4.22) com $F=1$.

A Figura 4.4.1 mostra a aplicação do esquema upwind semelhante ao dado em (4.23), $\operatorname{mas} \operatorname{com} H(u)=-\sqrt{1+u^{2}}$.

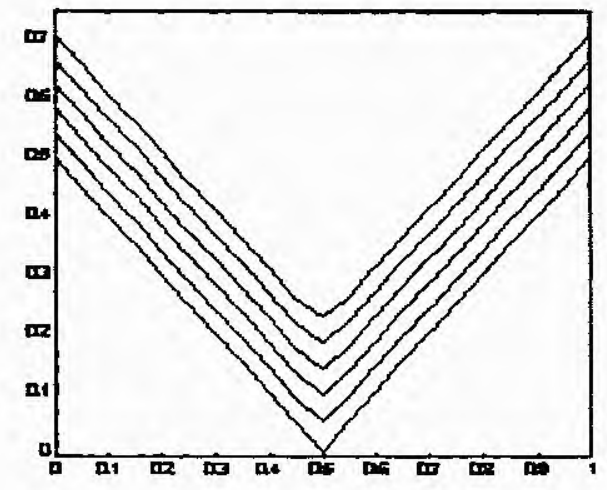

Figura 4.4.1. (a) Esquema com 20 pontos

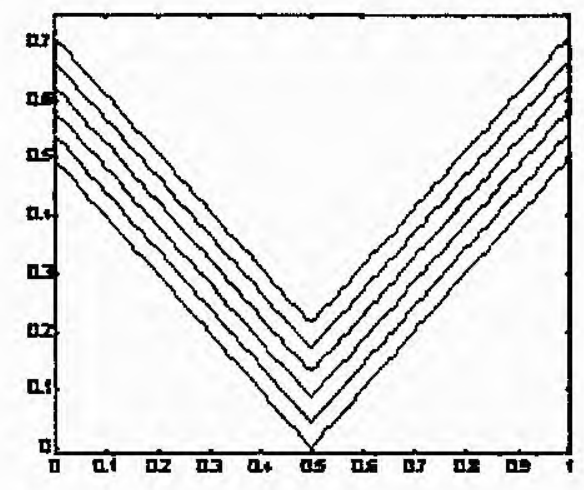

(b) Esquema com 100 pontos

Esquemas Numéricos para o Problema de Valor Inicial (4.17)

Nesta seção vamos utilizar alguns esquemas discutidos no Capítulo 3, para derivar esquemas para a equação H-J. Dada uma função velocidade convexa $F$ (isto é, uma função velocidade $F$ tal que o Hamiltoniano $H=F|\nabla U|$ é convexo), temos

(i) Esquema Convexo de Primeira Ordem:

$$
\phi_{i j k}^{n+1}=\phi_{i j k}^{n}-\Delta t\left[\max \left(F_{i j k}, 0\right) \nabla^{+}+\min \left(F_{i j k}, 0\right) \nabla^{-}\right]
$$


em que

$$
\begin{aligned}
\nabla^{+}= & {\left[\max \left(D_{i j k}^{-x}, 0\right)^{2}+\min \left(D_{i j k}^{+x}, 0\right)^{2}+\right.} \\
& \max \left(D_{i j k}^{-y}, 0\right)^{2}+\min \left(D_{i j k}^{+y}, 0\right)^{2}+ \\
& \left.\max \left(D_{i j k}^{-z}, 0\right)^{2}+\min \left(D_{i j k}^{+z}, 0\right)^{2}\right]^{1 / 2} \\
\nabla^{-}= & {\left[\max \left(D_{i j k}^{+x}, 0\right)^{2}+\min \left(D_{i j k}^{-x}, 0\right)^{2}+\right.} \\
& \max \left(D_{i j k}^{+y}, 0\right)^{2}+\min \left(D_{i j k}^{-y}, 0\right)^{2}+ \\
& \left.\max \left(D_{i j k}^{+z}, 0\right)^{2}+\min \left(D_{i j k}^{-z}, 0\right)^{2}\right]^{1 / 2} .
\end{aligned}
$$

Usamos uma notação resumida em que $D_{i}^{+x}$ significa $D^{+x} \phi_{i}^{n}$, etc.

\section{(ii) Esquema Convexo de Segunda Ordem:}

O esquema acima pode ser estendido para ordens mais altas. $O$ truque básico é construir um interruptor (função minmod) que liga-desliga quando um choque é detectado. Usamos uma aproximação de alta ordem para valores à esquerda e à direita do choque através de um polinômio de alta ordem usando uma construçāo ENO.

A idéia para o caso unidimensional é a seguinte. Considere o problema $U_{t}+H\left(U_{x}\right)=0$, em que a solução $U$ é conhecida em $t=t_{n}$. Integrando no intervalo $\left[t_{n}, t_{n+1}\right]$, temos

$$
U\left(x, t^{n+1}\right)=U\left(x, t^{n}\right)-\int_{0}^{\Delta t} H\left(U_{x}\left(x, t^{n}+s\right)\right) d s
$$

em que fizemos a mudança de variável $t=t^{n}+s$.

Seja $U_{i}^{n}$ a aproximação da solução exata no tempo $n \Delta t$. Desejamos descobrir uma função $R^{M}\left(x ; \mathbf{U}^{n}\right)$ que aproxime $U\left(x, t^{n}\right)$ em regiōes de suavidade de $U$, com ordem $o(\Delta x)^{M+1}$. Além do mais, esta função aproximadora deverá ser não-oscilatória mesmo se $U_{x}$ for descontínua, isto é, não será introduzido novas oscilações significantes.

\section{Construção ENO}

\section{Interpolação Essencialmente Nāo Oscilatória (ENO)}

Construiremos $R^{M}$ por indução sobre $M$. Em cada célula $x_{i} \leq x \leq x_{i+1}, R^{1}$ é definido como segue:

$$
R^{1}\left(x ; \mathbf{U}^{n}\right)=\mathbf{U}_{i}^{n}+\left(x-x_{i}\right) D^{+x} \mathbf{U}_{i}^{n}
$$




$$
=\mathrm{U}_{i}^{n}+\left(x-x_{i}\right) \mathrm{U}^{n}\left[x_{i}, x_{i+1}\right]
$$

em que a notação $v\left[x_{i-\mu}, \ldots, x_{i+\nu}\right]$ denota o coeficiente na interpolação polinomial de Newton.

Também definimos

$$
K_{\min }^{(1)}=i, \quad K_{\max }^{(1)}=i+1 .
$$

Suponha que definimos $R^{N-1}\left(x ; \mathbf{U}^{n}\right)$ para $x_{i} \leq x \leq x_{i+1}$ e também $K_{\min }^{(M-1)}$ e $K_{\max }^{(M-1)}$. Então calculamos

$$
\begin{aligned}
& a^{M}=\mathrm{U}^{n}\left[x_{K_{\min }^{(M-1)}}, \ldots, x_{K_{\max }^{(M-1)}+1}\right] \\
& b^{M}=\mathrm{U}^{n}\left[x_{K_{\min }^{(M-1)}-1}, \ldots, x_{K_{\max }^{(M-1)}}\right]
\end{aligned}
$$

e avançamos por indução.

Se $\left|a^{M}\right| \geq\left|b^{M}\right|$, então neste intervalo

$$
R^{M}\left(x ; \mathrm{U}^{n}\right)=R^{M-1}\left(x ; \mathrm{U}^{n}\right)+b^{M} \prod_{k=K_{\min }^{(M-1)}}^{K_{\max }^{(M-1)}}\left(x-x_{k}\right)
$$

com

$$
K_{\min }^{(M)}=K_{\min }^{(M-1)}-1 .
$$

Se $\left|a^{M}\right|<\left|b^{M}\right|$, então

$$
R^{M}\left(x ; \mathrm{U}^{n}\right)=R^{M-1}\left(x ; U^{n}\right)+a^{M} \prod_{k=K_{\min }^{(M-1)}}^{K_{\max }^{(M-1)}}\left(x-x_{k}\right)
$$

com

$$
K_{\max }^{(M)}=K_{\max }^{(M-1)}+1
$$

Assim em cada célula $x_{i} \leq x \leq x_{i+1}$, construímos um polinômio de grau $M$ que:

(1) interpola $\mathrm{U}_{\nu}^{n}$ em $M+1$ pontos consecutivos incluindo $x_{i}$ e $x_{i+1}$,

(2) minimiza a magnitude de todas as derivadas.

Pode ser mostrado (veja [20]) que, se $U(x)$ é $C_{0}^{\infty}$ por partes, ela possui no mínimo um número finito de saltos isolados em suas derivadas e é suave em $x=x_{0}$, então para $\Delta x$ suficientemente pequeno,

$$
\left[\left(\frac{d}{d x}\right)^{\nu} U(x)-\frac{d}{d x} R^{M}(x ; U)\right]_{x=x_{0}}=o(\Delta x)^{M-\nu+1}
$$


em que $\nu=0,1,2, \ldots, M$. Também temos que,

$$
T V\left[\frac{d}{d x} R^{M}(x ; U)\right] \leq T V\left[\frac{d}{d x} U(x)\right]+o(\Delta x)^{M} .
$$

Para continuar a descrição do algoritmo, resolvemos o problema de valor inicial

$$
\begin{aligned}
U_{t}+H\left(U_{x}\right) & =0 \\
\mathbf{U}(x, 0) & =R^{M}\left(x ; \mathbf{U}^{n}\right)
\end{aligned}
$$

exatamente (como no esquema de Godunov) ou aproximadamente, usando qualquer outra aproximação monótona. Para obter $\mathrm{U}^{n+1}$, definimos

$$
U_{i}^{n+1}=\int_{0}^{\Delta t} U_{t}\left(x_{i}, s\right) d s+U_{i}^{n}=U_{i}^{n}-\int_{0}^{\Delta t} H\left(U_{x}\left(x_{i}, s\right)\right) d s
$$

onde $\Delta t$ é tomado suficientemente pequeno.

Simplificamos este método para nossos cálculos. Primeiro, ao invés de resolver este problema exatamente, aproximamos o fluxo de Godunov pelo fluxo monótono mais simples $g^{\mathrm{HJ}}$.

$$
\frac{\partial}{\partial t} U_{i}=-g^{\mathrm{HJ}}\left(\frac{d}{d x} R^{N}\left(x_{i}^{-}, \mathrm{U}\right), \frac{d}{d x} R^{N}\left(x_{i}^{+}, \mathrm{U}\right)\right) .
$$

Para desenvolvermos o método numérico $\operatorname{com} N=2$, necessitaremos obter o polinômio interpolador de segunda ordem e sua derivada.

Se $\left|a^{2}\right| \geq\left|b^{2}\right|$

$$
\begin{aligned}
R^{2}(x ; U) & =U_{i}+U\left[x_{i}, x_{i+1}\right]\left(x-x_{i}\right)+U\left[x_{i-1}, x_{i}, x_{i+1}\right]\left(x-x_{i}\right)\left(x-x_{i+1}\right) . \\
\frac{d}{d x} R^{2}(x ; U) & =U\left[x_{i}, x_{i+1}\right]+U\left[x_{i-1}, x_{i}, x_{i+1}\right]\left(\left(x-x_{i+1}\right)+\left(x-x_{i}\right)\right) .
\end{aligned}
$$

Se $\left|a^{2}\right|<\left|b^{2}\right|$

$$
\begin{aligned}
R^{2}(x ; U) & =U_{i}+U\left[x_{i}, x_{i+1}\right]\left(x-x_{i}\right)+U\left[x_{i}, x_{i+1}, x_{i+2}\right]\left(x-x_{i}\right)\left(x-x_{i+1}\right) . \\
\frac{d}{d x} R^{2}(x ; U) & =U\left[x_{i}, x_{i+1}\right]+U\left[x_{i}, x_{i+1}, x_{i+2}\right]\left(\left(x-x_{i+1}\right)+\left(x-x_{i}\right)\right) .
\end{aligned}
$$

Substituindo $x_{i}$ e $x_{i+1}$ em (4.28), temos

$$
\begin{aligned}
\frac{d}{d x} R^{2}\left(x_{i} ; U\right) & =U\left[x_{i}, x_{i+1}\right]+U\left[x_{i-1}, x_{i}, x_{i+1}\right]\left(x_{i}-x_{i+1}\right) \\
& =\frac{U\left(x_{i+1}\right)-U\left(x_{i}\right)}{\Delta x}-\frac{\Delta x}{2}\left[\frac{U\left(x_{i+1}\right)-2 U\left(x_{i}\right)+U\left(x_{i-1}\right)}{\Delta x^{2}}\right]
\end{aligned}
$$




$$
\begin{aligned}
\frac{d}{d x} R^{2}\left(x_{i+1} ; U\right) & =U\left[x_{i}, x_{i+1}\right]+U\left[x_{i-1}, x_{i}, x_{i+1}\right]\left(x_{i+1}-x_{i}\right) \\
& =\frac{U\left(x_{i+1}\right)-U\left(x_{i}\right)}{\Delta x}+\frac{\Delta x}{2}\left[\frac{U\left(x_{i+1}\right)-2 U\left(x_{i}\right)+U\left(x_{i-1}\right)}{\Delta x^{2}}\right] .
\end{aligned}
$$

Substituindo $x_{i}$ e $x_{i+1}$ em (4.29), temos

$$
\begin{aligned}
\frac{d}{d x} R^{2}\left(x_{i} ; U\right) & =U\left[x_{i}, x_{i+1}\right]+U\left[x_{i}, x_{i+1}, x_{i+2}\right]\left(x_{i}-x_{i+1}\right) \\
& =\frac{U\left(x_{i+1}\right)-U\left(x_{i}\right)}{\Delta x}-\frac{\Delta x}{2}\left[\frac{U\left(x_{i+2}\right)-2 U\left(x_{i+1}\right)+U\left(x_{i-2}\right)}{\Delta x^{2}}\right] \\
\frac{d}{d x} R^{2}\left(x_{i+1} ; U\right) & =U\left[x_{i}, x_{i+1}\right]+U\left[x_{i}, x_{i+1}, x_{i+2}\right]\left(x_{i+1}-x_{i}\right) \\
& =\frac{U\left(x_{i+1}\right)-U\left(x_{i}\right)}{\Delta x}+\frac{\Delta x}{2}\left[\frac{U\left(x_{i+2}\right)-2 U\left(x_{i+1}\right)+U\left(x_{i}\right)}{\Delta x^{2}}\right] .
\end{aligned}
$$

Tomando $i=j-1$ em (4.31) e em (4.33), respectivamente, e denotando $\frac{d}{d x} R^{2}\left(x_{i+1} ; U\right)$ por $\frac{d}{d x} R^{2}\left(x_{j}^{+} ; U\right)$, obtemos

$$
\frac{d}{d x} R^{2}\left(x_{j}^{+} ; U\right)=D^{-x} U_{j}+\frac{\Delta x}{2} D^{-x-x} U_{j}
$$

ou

$$
\frac{d}{d x} R^{2}\left(x_{j}^{+} ; U\right)=D^{-x} U_{j}+\frac{\Delta x}{2} D^{-x+x} U_{j} .
$$

Denotando $\frac{d}{d x} R^{2}\left(x_{i} ; U_{i}\right)$ por $\frac{d}{d x} R^{2}\left(x_{i}^{-} ; U_{i}\right)$, obtemos

$$
\frac{d}{d x} R^{2}\left(x_{i}^{-} ; U\right)=D^{+x} U_{i}-\frac{\Delta x}{2} D^{+x-x} U_{i}
$$

ou

$$
\frac{d}{d x} R^{2}\left(x_{i}^{-} ; U\right)=D^{+x} U_{i}-\frac{\Delta x}{2} D^{+x+x} U_{i} .
$$

A seguir, substituindo em,

$$
\frac{\partial}{\partial t} U_{i}=-g_{\mathrm{H} J}\left(\frac{d}{d x} R^{2}\left(x_{i}^{-}, U\right), \frac{d}{d x} R^{2}\left(x_{i}^{+}, U\right)\right)
$$

e considerando a função minmod (4.43) para resolver as indecisões envolvendo $\frac{d}{d x} R^{2}\left(x_{i}^{-} ; U\right)$ e $\frac{d}{d x} R^{2}\left(x_{i}^{+} ; U\right)$, obtemos o esquema baseado em (4.24), (4.25) e (4.26), onde $\nabla^{+}$e $\nabla^{-}$são dados por

$$
\begin{aligned}
& \nabla^{+}=\left[\max (A, 0)^{2}+\min (B, 0)^{2}\right]^{1 / 2} \\
& \nabla^{-}=\left[\max (B, 0)^{2}+\min (A, 0)^{2}\right]^{1 / 2}, \quad \mathrm{e}
\end{aligned}
$$




$$
\begin{aligned}
& A=D_{i}^{-x}+\frac{\Delta x}{2} \operatorname{minmod}\left(D_{i j k}^{-x-x}, D_{i j k}^{-x+x}\right) \\
& B=D_{i}^{+x}-\frac{\Delta x}{2} \operatorname{minmod}\left(D_{i j k}^{+x+x}, D_{i j k}^{+x-x}\right) .
\end{aligned}
$$

Acabamos de descrever o caso unidimensional. Em seguida generalizamos e aplicamos o método para a função conjunto de nível $\phi_{i j k}^{n}$. Para mais detalhes veja [21] e [29].

$$
\phi_{i j k}^{n+1}=\phi_{i j k}^{n}-\Delta t\left[\max \left(F_{i j k}, 0\right) \nabla^{+}+\min \left(F_{i j k}, 0\right) \nabla^{-}\right]
$$

onde

$$
\begin{aligned}
\nabla^{+}= & {\left[\max (A, 0)^{2}+\min (B, 0)^{2}+\right.} \\
& \max (C, 0)^{2}+\min (D, 0)^{2}+ \\
- & \left.\max (E, 0)^{2}+\min (F, 0)^{2}\right]^{1 / 2} \\
\nabla^{-}= & {\left[\max (B, 0)^{2}+\min (A, 0)^{2}+\right.} \\
& \max (D, 0)^{2}+\min (C, 0)^{2}+ \\
& \left.\max (F, 0)^{2}+\min (E, 0)^{2}\right]^{1 / 2} \\
A= & D_{i j k}^{-x}+\frac{\Delta x}{2} m\left(D_{i j k}^{-x-x}, D_{i j k}^{+x-x}\right) \\
B= & D_{i j k}^{+x}-\frac{\Delta x}{2} m\left(D_{i j k}^{+x+x}, D_{i j k}^{+x-x}\right) \\
C= & D_{i j k}^{-y}+\frac{\Delta y}{2} m\left(D_{i j k}^{-y-y}, D_{i j k}^{+y-y}\right) \\
D= & D_{i j k}^{+y}-\frac{\Delta y}{2} m\left(D_{i j k}^{+y+y}, D_{i j k}^{+y-y}\right) \\
E= & D_{i j k}^{-z}+\frac{\Delta z}{2} m\left(D_{i j k}^{-z-z}, D_{i j k}^{+z-z}\right) \\
F= & D_{i j k}^{+z}-\frac{\Delta z}{2} m\left(D_{i j k}^{+z+z}, D_{i j k}^{+z-z}\right)
\end{aligned}
$$

e a função interruptora (minmod) é dada por

$$
m(x, y)=\left\{\begin{array}{cc}
\left\{\begin{array}{ll}
x & \text { se }|x| \leq|y| \\
y & \text { se }|x|>|y|
\end{array}\right\} & x y \geq 0 \\
0 & x y<0
\end{array}\right.
$$




\subsection{Esquemas para Funções Velocidades Não-Convexas}

Dada uma função velocidade não convexa $F$, isto é, uma função velocidade $F$ tal que o Hamiltoniano resultante $H=F|\nabla U|$ é não-convexo, podemos estender nossos esquemas em uma variedade de maneiras. Um conjunto de tais extensōes foi introduzido por Osher e Shu [30]. Esses esquemas resultam de substituir o Hamiltoniano $F|\nabla \phi|$ pela função de fluxo numérico de Lax-Friedrichs.

Começamos com o problema de valor inicial na forma

$$
\begin{cases}u_{t}+H\left(u_{x}\right)=0, & \text { para } t>0, x \in \mathbb{R} \\ u(x, 0)=u_{0}(x), & \text { para } x \in \mathbb{R}\end{cases}
$$

Consideramos o esquema

$$
U_{i}^{n+1}=U_{i}^{n}-\Delta t\left\{H\left(\frac{U_{i+1}^{n}-U_{i-1}^{n}}{2 \Delta x}\right)-\frac{\theta}{R} \frac{U_{i+1}^{n}-2 U_{i}^{n}+U_{i-1}^{n}}{\Delta x}\right\},
$$

onde $\theta>0$ é dado. Esse esquema é derivado de (4.44) adicionando o termo parabólico $\epsilon u_{x x}$ à direita dessa equação (como fizemos anteriormente) e discretizando-se por diferenças

centrais. Temos que, $\epsilon=\frac{(\Delta x)^{2}}{\Delta t} \theta$ e quando $\Delta x \rightarrow 0$, resulta em $\epsilon \rightarrow 0$. A relação (4.45) pode ser reescrita como

$$
U_{i}^{n+1}=U_{i}^{n}-\Delta t\left\{H\left(\frac{D^{+x} U_{i}^{n}+D^{-x} U_{i}^{n}}{2}\right)-\frac{\theta}{R}\left(D^{+x} U_{i}^{n}-D^{-x} U_{i}^{n}\right)\right\}
$$

e portanto a função de fluxo numérico desse esquema é

$$
g(\alpha, \beta)=H\left(\frac{\beta+\alpha}{2}\right)-\frac{(\beta-\alpha)}{R} \theta \quad \alpha, \beta \in \mathbb{R}
$$

Claramente $g(\alpha, \alpha)=H(\alpha)$ e portanto (4.45) é consistente. O esquema é monótono em $[-R, R]$ se $1-2 \theta \geq 0$ (monotonicidade em $U_{i}^{n}$ ), e $\theta-R\left|H^{\prime}(\alpha)\right| / 2 \geq 0$ para $\alpha \leq R$ (monotonicidade em $\left.U_{i+1}^{n}, U_{i-1}^{n}\right)$. Este esquema é similar ao esquema de Lax-Friedrichs para leis de conservação unidimensionais, pois ambos são conservativos, e para $\theta=1 / 2$ eles possuem a mesma condição de monotonicidade.

Se $N>1$, a relação entre equações de Hamilton-Jacobi e leis de conservação desaparecem no sentido que não conseguimos preservar a mesma ordem quando transformamos uma equação de Hamilton-Jacobi em uma lei de conservação como ocorre para o caso unidimen- 
sional. Para $N=2$ apresentamos o análogo do esquema de Lax-Friedrichs, isto é,

$$
\begin{aligned}
U_{i j}^{n+1}= & U_{i j}^{n}-\Delta t H\left(\frac{U_{i+1 j}^{n}-U_{i-1 j}^{n}}{2 \Delta x}, \frac{U_{i j+1}^{n}-U_{i j-1}^{n}}{2 \Delta y}\right) \\
& +\Delta t\left\{\frac{\theta}{\lambda^{x}}\left(\frac{U_{i+1 j}^{n}-2 U_{i j}^{n}+U_{i-1 j}^{n}}{\Delta x}\right)+\frac{\theta}{\lambda^{y}}\left(\frac{U_{i j+1}^{n}-2 U_{i j}^{n}+U_{i j-1}^{n}}{\Delta y}\right)\right\} \\
= & U_{i j}^{n}-\Delta t H\left(\frac{D^{+x} U_{i j}^{n}+D^{-x} U_{i j}^{n}}{2}, \frac{D^{+y} U_{i j}^{n}+D^{-y} U_{i j}^{n}}{2}\right) \\
& +\theta \Delta x\left(D^{+x} U_{i j}^{n}-D^{-x} U_{i j}^{n}\right)+\theta \Delta y\left(D^{+y} U_{i j}^{n}-D^{-y} U_{i j}^{n}\right)
\end{aligned}
$$

onde $\lambda^{x}=\frac{\Delta t}{\Delta x}$ e $\lambda^{y}=\frac{\Delta t}{\Delta y}$. Esse esquema também é monótono sobre $[-R, R]$ contanto que $0<\theta<1 / 4, \quad \theta-\lambda^{x}\left|H_{1}^{\prime}(\alpha, \beta)\right| \geq 0$ e $\theta-\lambda^{y}\left|H_{2}^{\prime}(\alpha, \beta)\right| \geq 0$ para $|\alpha|,|\beta| \leq R$, onde $H_{i}^{\prime}$ denota a derivada de $H$ com relação ao i-ésimo argumento. Para o método de conjunto de nível com Hamiltoniano $H(u, v, w)$, a equação (4.14) aproximada por (4.15) possui versão multidimensional da função de fluxo numérico de Lax-Friedrichs, $g^{\text {LF }}$, dada por

$$
\begin{aligned}
g^{\mathrm{LF}}\left(u_{1}, u_{2}, v_{1}, v_{2}, w_{1}, w_{2}\right)= & -\frac{\Delta x}{2 \Delta t}\left(u_{2}-u_{1}\right)+\frac{1}{2}\left[H\left(u_{2}\right)-H\left(u_{1}\right)\right] \\
& -\frac{\Delta y}{2 \Delta t}\left(v_{2}-v_{1}\right)+\frac{1}{2}\left[H\left(v_{2}\right)-H\left(v_{1}\right)\right] \\
& -\frac{\Delta z}{2 \Delta t}\left(w_{2}-w_{1}\right)+\frac{1}{2}\left[H\left(w_{2}\right)-H\left(w_{1}\right)\right]
\end{aligned}
$$

para obter os seguintes esquemas:

(i) Esquema de Primeira Ordem Não-Convexo na Variável Espacial:

$$
\begin{aligned}
\phi_{i j k}^{n+1}= & \phi_{i j k}^{n}-\Delta t\left[H\left(\frac{D_{i j k}^{-x}+D_{i j k}^{+x}}{2}, \frac{D_{i j k}^{-y}+D_{i j k}^{+y}}{2}, \frac{D_{i j k}^{-z}+D_{i j k}^{+z}}{2}\right)-\frac{1}{2} \alpha_{u}\left(D_{i j k}^{+x}-D_{i j k}^{-x}\right)\right. \\
& \left.-\frac{1}{2} \alpha_{v}\left(D_{i j k}^{+y}-D_{i j k}^{-y}\right)-\frac{1}{2} \alpha_{w}\left(D_{i j k}^{+z}-D_{i j k}^{-z}\right)\right]
\end{aligned}
$$

onde $\alpha_{u} \quad\left(\alpha_{v}, \alpha_{w}\right)$ é um limitante para a derivada parcial com respeito ao primeiro (segundo, terceiro) argumento, e o Hamiltoniano não-convexo é uma função dada pelo problema que se quer resolver.

(ii) Esquema de Segunda Ordem Não-Convexo na Variável Espacial:

$$
\begin{aligned}
\phi_{i j k}^{n+1}= & \phi_{i j k}^{n}-\Delta t\left[H\left(\frac{A+B}{2}, \frac{C+D}{2}, \frac{E+F}{2}\right)-\frac{1}{2} \alpha_{u}(B-A)\right. \\
& \left.-\frac{1}{2} \alpha_{v}(D-C)-\frac{1}{2} \alpha_{w}(F-E)\right],
\end{aligned}
$$

onde $A, B, C, D, E$, e $F$ são definidas em (4.37)-(4.42). 
Para o problema de valor de fronteira, podemos derivar esquemas de maneira similar.

Assim, do que vimos acima, podemos resumir da seguinte maneira:

- Se o Hamiltoniano "H"é convexo, então usamos a função de fluxo numérico $g^{\mathrm{HJ}}$ dada em (4.16) e apresentada em [29].

- Se o Hamiltoniano "H"é não-convexo, então usamos a função de fluxo numérico $g^{\mathrm{LF}}$ dada em (4.49) e apresentada em [8].

\subsection{O Problema de Valor de Fronteira: Método Esta- cionário}

Nesta seção apresentamos esquemas de primeira e segunda ordens para o problema de valor de fronteira estacionário. Lembramos que desejamos resolver

$$
|\nabla T| F(x, y)=1 \quad \text { tal que } \quad \Gamma=\{(x, y) / T(x, y)=0\}
$$

onde $\Gamma$ é a posição inicial da interface. Substituindo o Hamiltoniano numérico como fizemos anteriormente, podemos produzir os seguintes esquemas:

(i) Esquema de primeira ordem convexo na variável espacial:

$$
\begin{array}{r}
{\left[\max \left(D_{i j k}^{-x} T, 0\right)^{2}+\min \left(D_{i j k}^{+x} T, 0\right)^{2}+\max \left(D_{i j k}^{-y} T, 0\right)^{2}+\min \left(D_{i j k}^{+y} T, 0\right)^{2}\right.} \\
\left.+\max \left(D_{i j k}^{-z} T, 0\right)^{2}+\min \left(D_{i j k}^{+z} T, 0\right)^{2}\right]^{1 / 2}=\frac{1}{F_{i j k}}
\end{array}
$$

(ii) Esquema de segunda ordem convexo na variável espacial:

$$
\begin{aligned}
{\left[\max (A, 0)^{2}+\right.} & \min (B, 0)^{2}+\max (C, 0)^{2}+\min (D, 0)^{2} \\
& \left.+\max (E, 0)^{2}+\min (F, 0)^{2}\right]^{1 / 2}=\frac{1}{F_{i j k}}
\end{aligned}
$$

onde $A, B, C, D, E$, e $F$ são definidos (4.37)-(4.38).

\subsection{Aproximações para as Variáveis Geométricas}

Uma vantagem da perspectiva das equaçōes diferenciais parciais é que propriedades geométricas da interface, tais como curvatura e direção normal, são calculadas facilmente. Por 
exemplo, considere uma curva propagando no plano, formulada como um problema de valor inicial. A expressão para a curvatura do conjunto de nível zero (e também para os demais níveis) é dada por

$$
\kappa=\nabla \cdot \frac{\nabla \phi}{|\nabla \phi|}=\frac{\phi_{x x} \phi_{y}^{2}-2 \phi_{y} \phi_{x} \phi_{x y}+\phi_{y y} \phi_{x}^{2}}{\left(\phi_{x}^{2}+\phi_{y}^{2}\right)^{3 / 2}}
$$

No caso de uma superfície propagando no espaço $\mathbb{R}^{3}$, temos outras definições para a curvatura da frente, incluindo a curvatura média $\kappa_{\mathrm{M}}$ e a curvatura Gaussiana $\kappa_{\mathrm{G}}$. Ambas podem ser expressas em termos da função conjunto de nível $\phi$.

$$
\begin{gathered}
\kappa_{\mathrm{M}}=\nabla \cdot \frac{\nabla \phi}{|\nabla \phi|}=\frac{\left\{\begin{array}{c}
\left(\phi_{y y}+\phi_{z z}\right) \phi_{x}^{2}+\left(\phi_{x x}+\phi_{z z}\right) \phi_{y}^{2}+\left(\phi_{x x}+\phi_{z z}\right) \phi_{z}^{2} \\
-2 \phi_{x} \phi_{y} \phi_{x y}-2 \phi_{x} \phi_{z} \phi_{x z}-2 \phi_{y} \phi_{z} \phi_{y z}
\end{array}\right\}}{\left(\phi_{x}^{2}+\phi_{y}^{2}+\phi_{z}^{2}\right)^{3 / 2}} \\
\kappa_{\mathrm{G}}=\frac{\left\{\begin{array}{c}
\left(\phi_{y y} \phi_{z z}-\phi_{y z}^{2}\right) \phi_{x}^{2}+\left(\phi_{x x} \phi_{z z}-\phi_{x z}^{2}\right) \phi_{y}^{2}+\left(\phi_{x x} \phi_{y y}-\phi_{x y}^{2}\right) \phi_{z}^{2} \\
+2\left[\phi_{x} \phi_{y}\left(\phi_{x z} \phi_{y z}-\phi_{z z}\right)+\phi_{y} \phi_{z}\left(\phi_{x y} \phi_{x z}-\phi_{y z} \phi_{x x}\right)+\phi_{x} \phi_{z}\left(\phi_{x y} \phi_{y z}-\phi_{x z} \phi_{y y}\right)\right]
\end{array}\right\}}{\left(\phi_{x}^{2}+\phi_{y}^{2}+\phi_{z}^{2}\right)^{2}} .
\end{gathered}
$$

Se $\phi$ é suave, então o vetor normal no ponto $\left(x_{0}, y_{0}\right)$ à curva de nível passando por $\left(x_{0}, y_{0}\right)$ é $\nabla \phi\left(x_{0}\right) /\left|\nabla \phi\left(x_{0}\right)\right|$. Entretanto, quando $\phi$ desenvolve cantos devemos ser cuidadosos na construção do vetor normal. É necessário um esquema mais sofisticado do que apenas construir uma aproximação de diferenças para $\nabla \phi$. Sethian e Strain [40], sugeriu a seguinte técnica. Primeiro, são feitas aproximações de diferenças não centradas em cada possível direção. Todos os quatro limitantes normais são somados e ponderados para produzir uma aproximação para o vetor normal no canto, veja a Figura 4.7.1. Assim, o vetor normal $\vec{n}_{i j}$ é formado tomando primeiro

$$
\begin{aligned}
n_{i j}^{*} \equiv & \frac{\left(\phi_{x}, \phi_{y}\right)}{\left(\phi_{x}^{2}+\phi_{y}^{2}\right)^{1 / 2}} \\
\approx & \frac{1}{4}\left[\frac{\left(D_{i j}^{+x}, D_{i j}^{+y}\right)}{\left[\left(D_{i j}^{+x}\right)^{2}+\left(D_{i j}^{+y}\right)^{2}\right]^{1 / 2}}+\frac{\left(D_{i j}^{-x}, D_{i j}^{+y}\right)}{\left[\left(D_{i j}^{-x}\right)^{2}+\left(D_{i j}^{+y}\right)^{2}\right]^{1 / 2}}\right. \\
& \left.+\frac{\left(D_{i j}^{+x}, D_{i j}^{-y}\right)}{\left[\left(D_{i j}^{+x}\right)^{2}+\left(D_{i j}^{-y}\right)^{2}\right]^{1 / 2}}+\frac{\left(D_{i j}^{-x}, D_{i j}^{-y}\right)}{\left[\left(D_{i j}^{-x}\right)^{2}+\left(D_{i j}^{-y}\right)^{2}\right]^{1 / 2}}\right]
\end{aligned}
$$


e então normalizando temos $n_{i j} \equiv \frac{n_{i j}^{*}}{\left|n_{i j}^{*}\right|}$. Se qualquer uma das aproximações não centradas para $|\nabla \phi|$ se anular, este termo não é considerado e os pesos são ajustados de acordo.

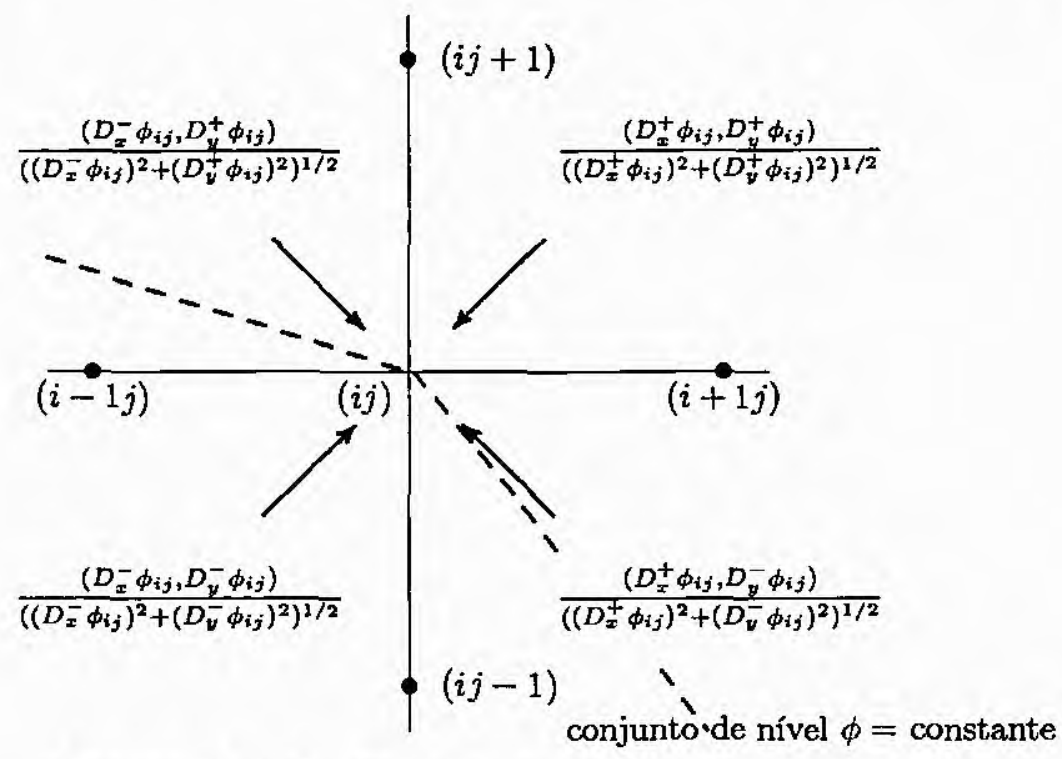

Figura 4.7.1. Construção do método modificado.

Acabamos de mostrar aproximações para o cálculo das propriedades geométricas, curvaturas e direções normais, e vimos que não são muito complicadas. Agora mostraremos outras duas propriedades geométricas, medição de grandezas (área, comprimento), em conjuntos de níveis. Para uma região aberta $\Omega$ qualquer, com fronteira suave, assumimos a existência de uma função conjunto de nível $\phi$, Lipschitz contínua, satisfazendo

$$
\begin{aligned}
& \phi(x, y)>0 \text { para }(x, y) \in \Omega \\
& \phi(x, y)=0 \text { para }(x, y) \in \partial \Omega \\
& \phi(x, y)<0 \text { para }(x, y) \in \tilde{\Omega}^{c} .
\end{aligned}
$$

Então temos as seguintes duas expressões para a área e comprimento da fronteira, a saber,

$$
\begin{aligned}
\text { comprimento }(\partial \Omega) & =\iint \delta(\phi(x, y))|\nabla \phi(x, y)| d x d y \\
\text { área }(\Omega) & =\iint H(\phi(x, y)) d x d y
\end{aligned}
$$

onde $H$ é a função de Heaviside, $H(x)=\left\{\begin{array}{ll}1 & \text { se } x \geq 0 \\ 0 & \text { se } x<0,\end{array}\right.$ e $\delta$ é a função delta de Dirac, $\delta(x)=\frac{d}{d x} H(x) \quad$ (no sentido de distribuições). 


\section{Capítulo 5}

\section{Resultados e Considerações Finais}

Neste capítulo são apresentados resultados obtidos pela implementação dos esquemas numéricos de primeira e de segunda ordem, (4.24) e (4.34), respectivamente, descritos no capítulo anterior e utilizados para aproximar o problema de valor inicial

$$
\begin{gathered}
\phi_{t}+F|\nabla \phi|=0 \\
\Gamma(t)=\{(x, y): \phi(x, y, t)=0\}
\end{gathered}
$$

para diferentes formas da função $F$. Por exemplo, imaginamos uma curva inicial evoluindo através de dois tipos de movimentos diferentes e simultâneos. Primeiro, através de uma velocidade escalar $F_{0}$ em sua direção normal. Segundo, sendo transportada (advecção) por um campo velocidade $\bar{U}(x, y, t)$ cuja direção e intensidade dependem da posição e do tempo, e não dela mesma. Assim sendo, temos que $F=F_{\text {prop }}+F_{\text {adv }}$, em que $F_{\text {prop }}=F_{0}$ é a velocidade de propagação, e $F_{\text {adv }}=\bar{U}(x, y, t) . \vec{n}$ é a velocidade de advecção, em que $\vec{n}=\nabla \phi /|\nabla \phi|$. Dessa maneira, a equação de conjunto de nível (5.1) pode ser reescrita como:

$$
\phi_{t}+F_{0}|\nabla \phi|+\bar{U}(x, y, t) \cdot \nabla \phi=0 .
$$

Também fizemos algumas comparações com outros esquemas já mencionados com objetivo de destacar a eficiência dos esquemas de conjuntos de níveis.

\subsection{Transporte de uma Frente Através de um Campo Parabólico}

Neste exemplo, transportamos uma frente inicial através de um campo parabólico e observamos a atuação desse campo à medida que a frente é transportada. 
A Figura 5.1.1 abaixo, ilustra como-inicialmente pensamos em apresentar esse exemplo. Consideramos uma frente circular de centro $\left(x_{0}, y_{0}\right)=(7 / 12,0.5)$ e de raio $r=1 / 3$ situada no interior de um domínio computacional retangular dado por $\left\{(x, y) \in \mathbb{R}^{2}: 0 \leq x \leq 2,0 \leq\right.$ $y \leq 1\}$ para ser transportada pelo campo parabólico $C(y)=y(1-y)$. $\AA$ medida que o círculo sofre a ação desse campo, podemos prever que a deformação não deve ser uniforme e que pontos numa vizinhança de $(1 / 4,1 / 2)$ e de $(11 / 12,1 / 2)$, ou seja, pontos laterais, serão transportados com maior velocidade que os demais. A seguir ilustramos essa situação.

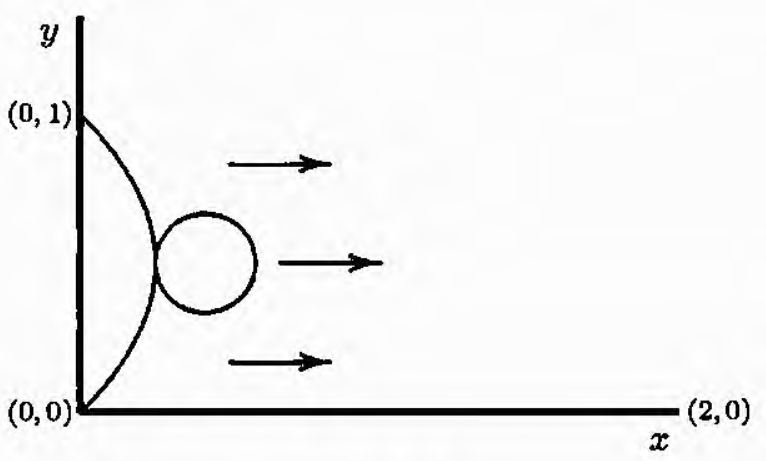

Figura 5.1.1.

Para transportarmos esse círculo fizemos uso do problema de valor inicial

$$
\begin{gathered}
\phi_{t}+F|\nabla \phi|=0 \\
\phi(x, y, 0)=(x-7 / 12)^{2}+(y-1 / 2)^{2}-\left(\frac{1}{3}\right)^{2}
\end{gathered}
$$

e do esquema numérico explícito de primeira ordem (4.24). A função velocidade $F$ é a advecção passiva e é dada por $F=F_{\text {adv }}=\vec{U}(x, y, t) \cdot \frac{\nabla \phi}{|\nabla \phi|}$. Utilizamos o campo de velocidade dado por $\vec{U}(x, y, t)=(\vec{u}(x, y, t), \vec{v}(x, y, t))=(y(1-y), 0)$.

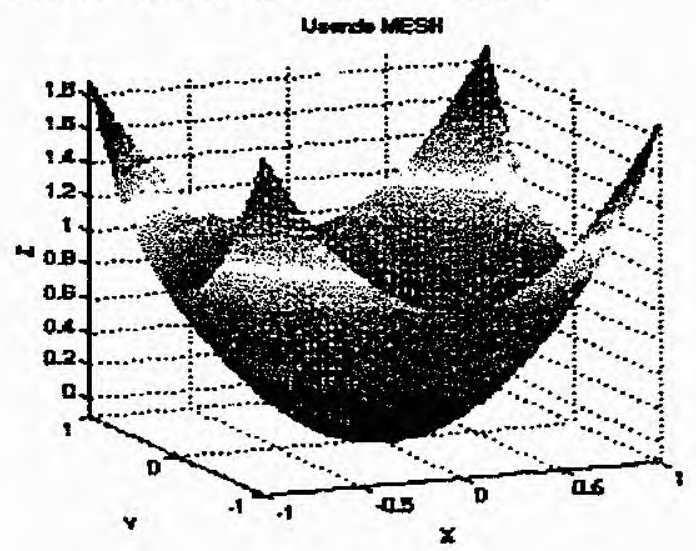

Figura 5.1.2. Parabolóide. 
A solução do PVI é uma função $\phi=\phi(x, y, t)$ tal que $\phi(x, y, 0)=(x-7 / 12)^{2}+(y-1 / 2)^{2}-$ $\left(\frac{1}{3}\right)^{2}$. O conjunto de pontos formado por $\phi(x, y, 0)=0$ é o círculo de centro $(7 / 12,1 / 2) \mathrm{e}$ raio $r=1 / 3$. Para que as famílias de círculos (subconjuntos do $\mathbb{R}^{2}$ ) sejam o conjunto de nível zero de uma função de dimensão maior $\phi$, consideramos o seguinte subconjunto $\left\{(x, y, z) \in \mathbb{R}^{3}: z=\phi(x, y, t), t\right.$ é um parâmetro $\} \subset \mathbb{R}^{3}$, que nesse caso são parabolóides (Figura 5.1.2), e fazemos $\phi(x, y, t)=0$.

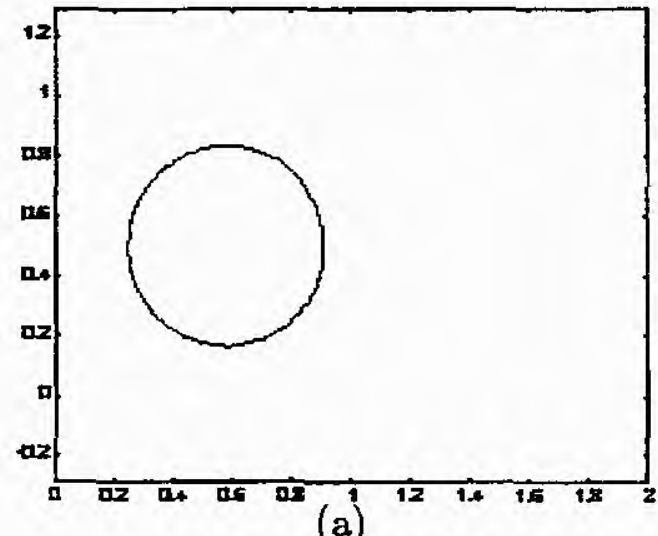

(a)
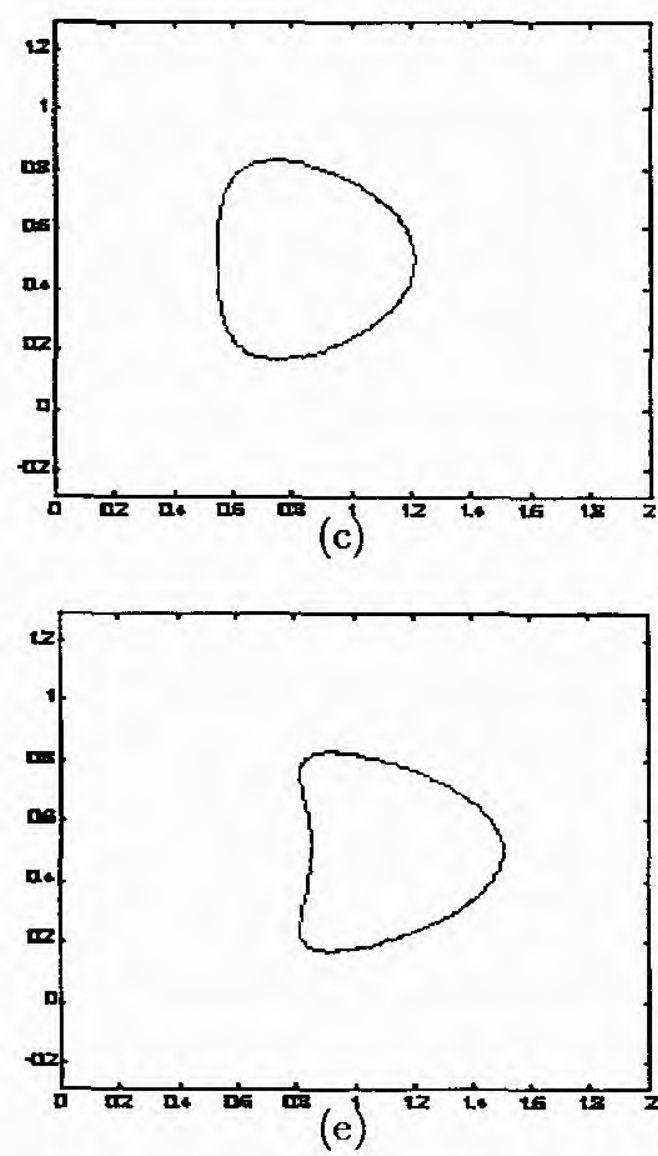

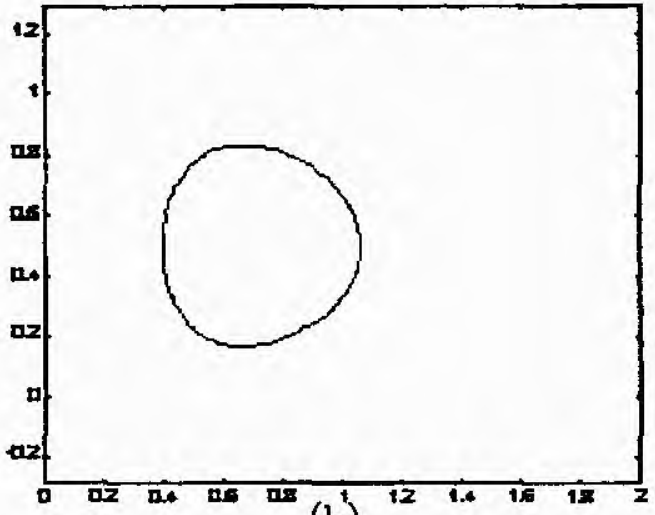

(b)
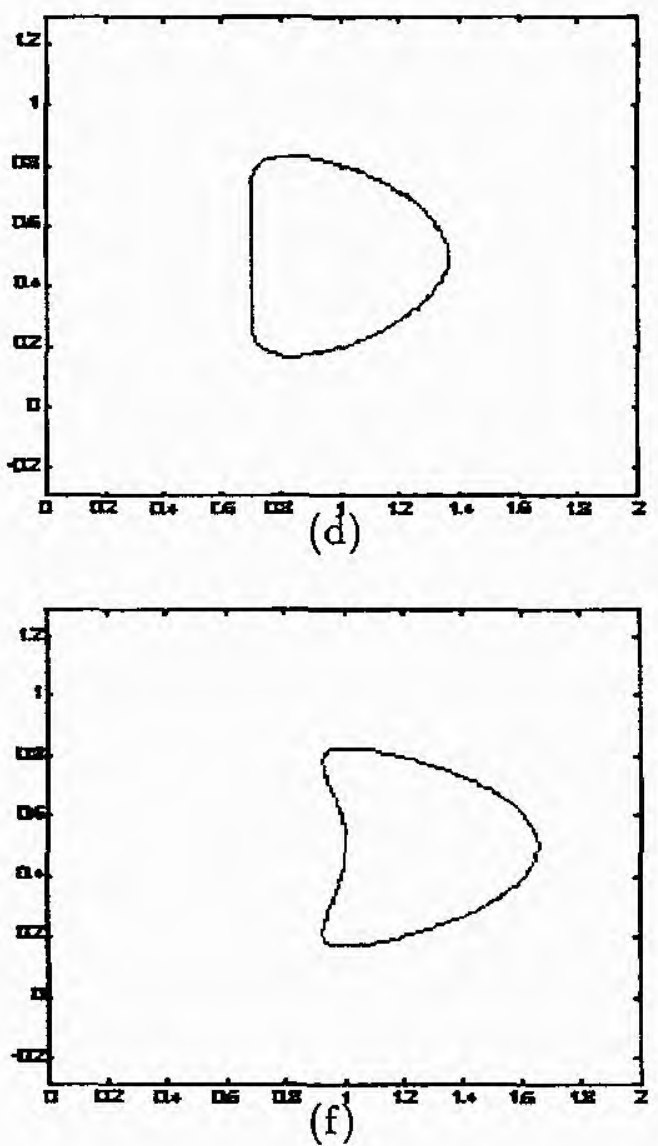

Figura 5.1.3. Transporte de uma frente circular usando um campo parabólico para os tempos: (a) $t=0$; (b) $t=0.6$; (c) $t=1.2$; (d) $t=1.8$; (e) $t=2.4$; (f) $t=3$, utilizando o esquema numérico (4.24). 
Na Figura 5.1.3 pode-se visualizar o comportamento dos círculos sob a ação do campo parabólico. Inicialmente na forma circular, o círculo vai se deformando durante o transporte assumindo a forma parabólica já esperada. Chama-se atenção para us pontos laterais do círculo que se propagam com maior velocidade em relação aos pontos superiores e inferiores.

\subsection{Transporte da Curva Cosseno}

Neste exemplo, transportamos a curva cosseno $\gamma(s)=(1-s,[1+\cos (2 \pi s)] / 2)$ com velocidade de propagação $F=1$ usando o esquema convexo de primeira ordem (4.24) para o problema de valor inicial (5.1). Na Seção 2.3 do Capítulo 2 fizemos esse transporte utilizando a equação paramétrica de movimento (2.2) e constatamos que a frente produziu auto interseção ao propagar-se. O esquema de conjuntos de níveis removeu essas interseções espúrias e preservou as singularidades que realmente surgem na propagação das curvas.

Para aplicarmos o esquema de conjunto de nível (4.24) para a curva cosseno precisaremos primeiro eliminar o parâmetro $s$ e transformá-la numa função $y=y(x)$ e em seguida em uma funçāo de duas variáveis. Assim, seja $x=1-s$ e portanto $y=\frac{1+\cos (2 \pi(1-x))}{2}$. O problema de valor inicial que passamos a resolver é:

$$
\begin{gathered}
\phi_{t}+|\nabla \phi|=0 \\
\phi(x, y, 0)=y-\frac{1+\cos (2 \pi(1-x))}{2} .
\end{gathered}
$$

Para obtermos a curva cosseno inicial basta considerarmos o nível zero da superfície dada pela função $\phi(x, y, 0)=y-\frac{1+\cos (2 \pi(1-x))}{2}$. Seu gráfico é o conjunto $G_{\phi}=\left\{(x, y, z) \in \mathbb{R}^{3}\right.$ : $z=\phi(x, y, 0)\} \subset \mathbb{R}^{3}$, dado pela Figura 5.2.1 abaixo

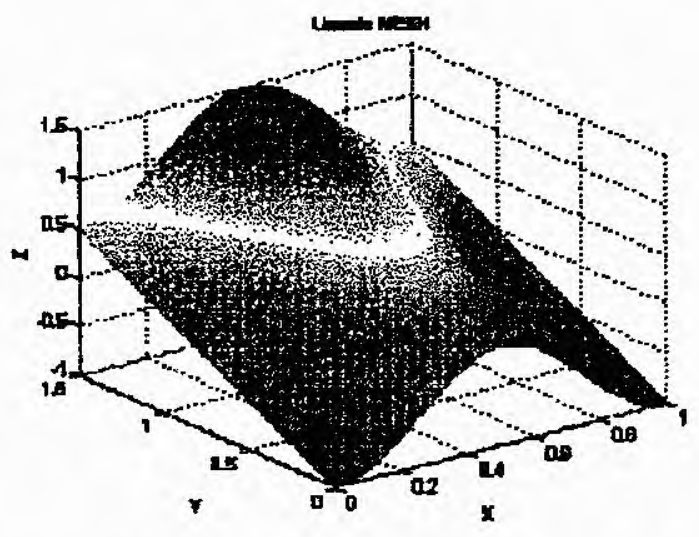

Figura 5.2.1. Gráfico de $\phi$. 
Podemos observar na Figura 5.2.2 todo o processo de propagação da curva cosseno, bem como a eliminação de auto interseçōes conforme mostrado na Figura 2.3.3, e a preservação de singularidades conforme a cvolução.

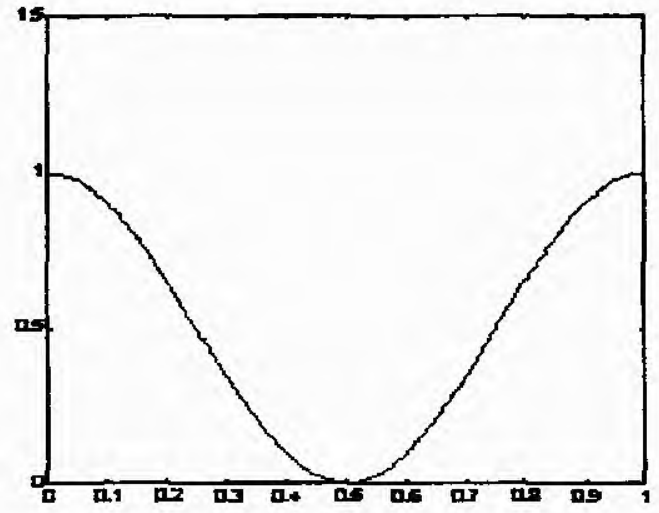

(a)

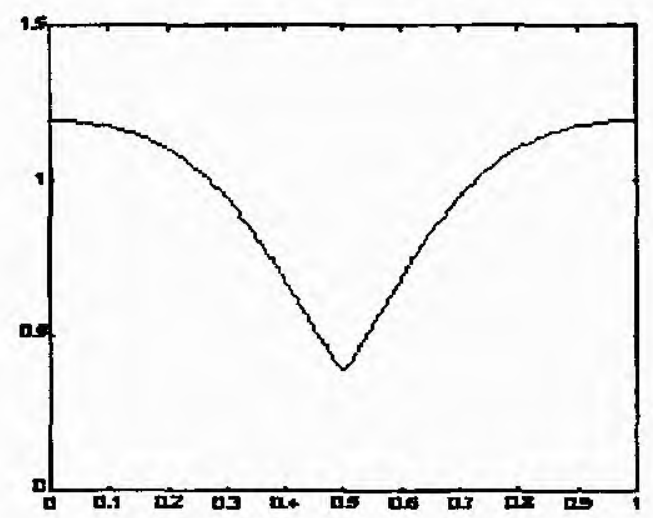

(c)

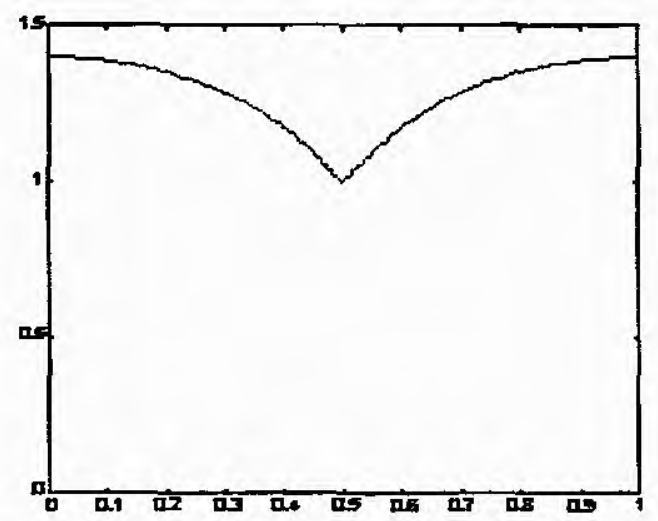

(e)

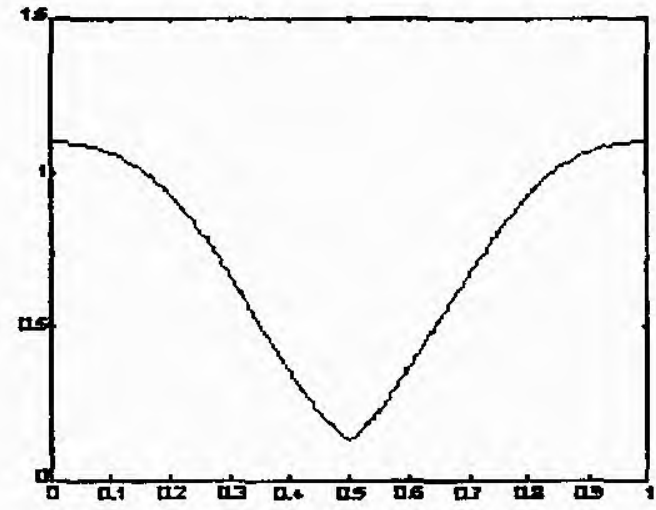

(b)

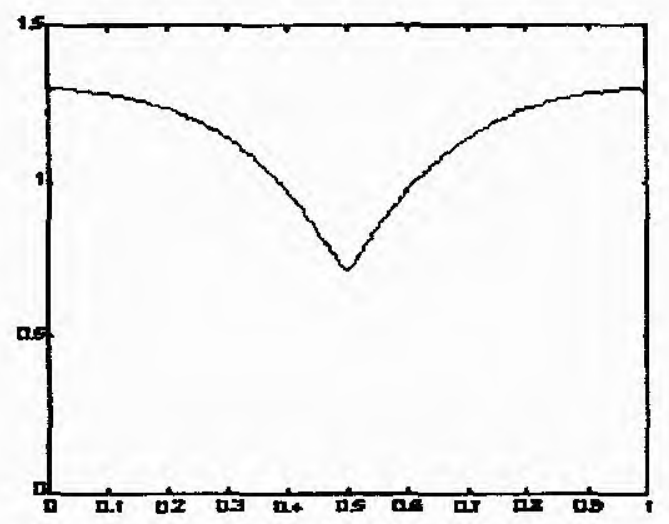

(d)

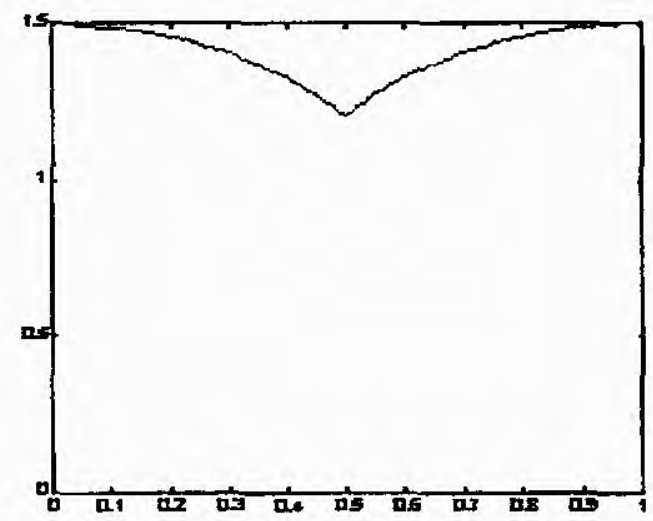

(f)

Figura 5.2.2. Transporte da curva cosseno usando $F=1$ para os tempos: (a) $t=0$; (b) $t=0.1$; (c) $t=0.2$; (d) $t=0.3$; (e) $t=0.4$; (f) $t=0.5$, utilizando o esquema (4.24). 


\subsection{Rotação de um Círculo Utilizando Esquemas de Primeira e Segunda Ordem}

Neste exemplo, consideramos a rotação de um círculo de raio $1 / 4$ centrado no ponto $(-0.5,0)$. Usamos um campo de velocidade $(u, v)=(y,-x)$, de maneira que estamos interessados em resolver (5.1) com $F=(y,-x) \cdot \frac{\nabla \phi}{|\nabla \phi|}$. Na Figura 5.3 .1 mostramos o movimento deste círculo para vários refinamentos da malha através de esquemas de primeira e segunda ordem dados em (4.24) e (4.34), respectivamente, que discretizam (5.1).

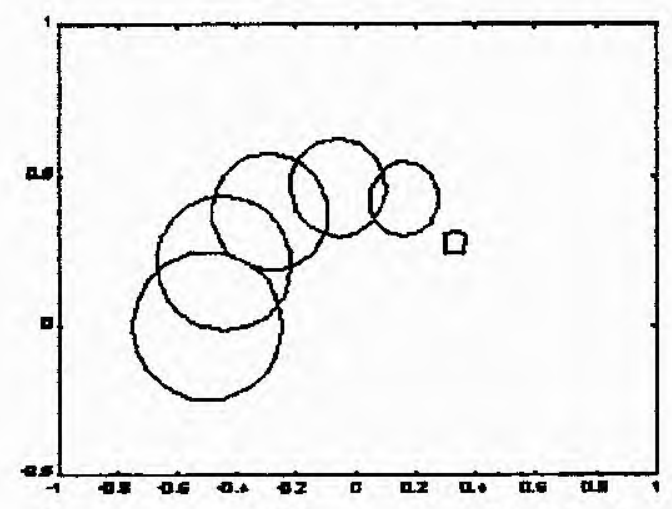

$\Delta x=\Delta y=0.05, \Delta t=0.03:$ Ordem 1

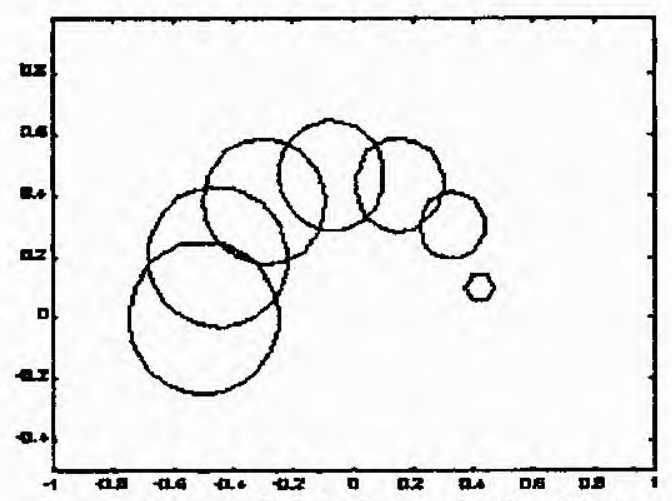

$\Delta x=\Delta y=0.04, \Delta t=0.02:$ Ordem 1

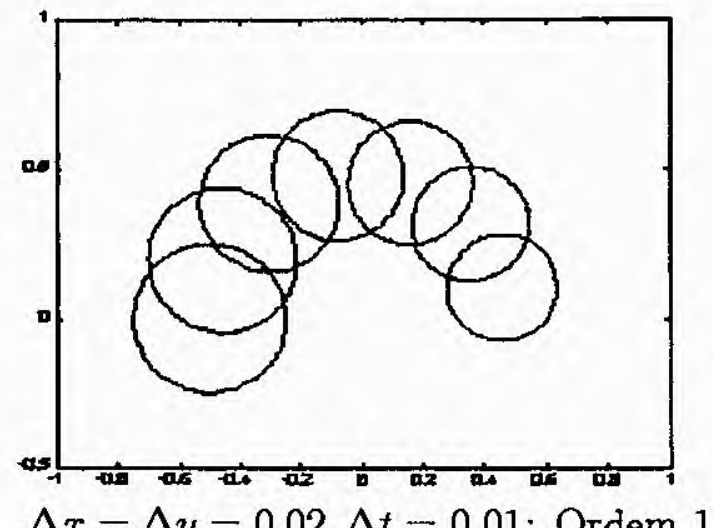

$\Delta x=\Delta y=0.02, \Delta t=0.01:$ Ordem 1

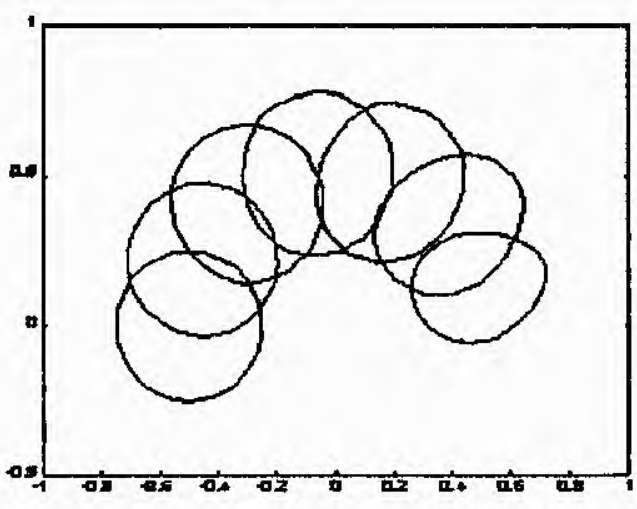

$\Delta x=\Delta y=0.05, \Delta t=0.03:$ Ordem 2

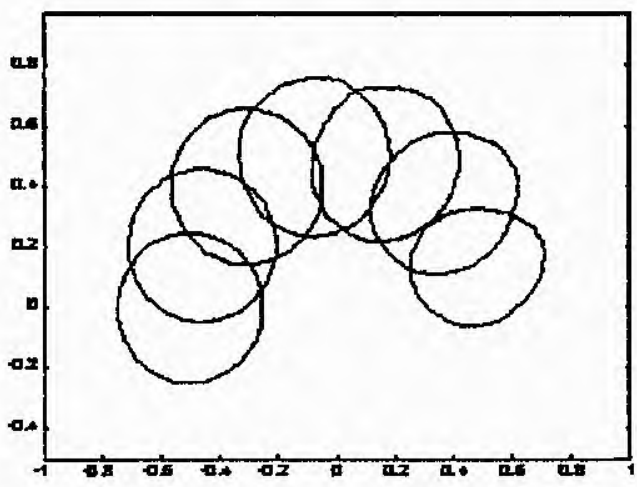

$\Delta x=\Delta y=0.04, \Delta t=0.02:$ Ordem 2

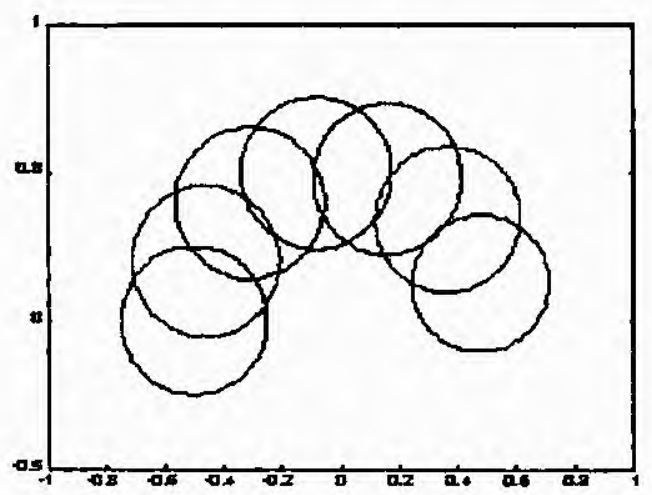

$\Delta x=\Delta y=0.02, \Delta t=0.01:$ Ordem 2

Figura 5.3.1. Rotação de um círculo para diferentes refinamentos da malha. 
Esse exemplo ilustra a importância na utilização de métodos precisos para o transporte de interfaces. Note como o método de primeira ordem perde massa ao longo do tempo.

Quando a malha é pouco rcfinada, ocorre uma considerável difusão. À medida que a malha é refinada podemos notar uma conservação da massa para ambos os esquemas, mas principalmente para o esquema de segunda ordem.

O círculo é rotacionado até $t=3.0$ e plotado nos tempos $t=0.0, t=0.5, t=1.0, t=1.5$, $t=2.0, t=2.5, t=3.0$.

\subsection{Considerações Finais}

Esta seção apresenta de maneira sucinta as considerações finais e algumas perspectivas futuras que poderão ser realizadas como consequência dos estudos contidos neste trabalho de mestrado.

\subsubsection{Conclusões}

Conforme apresentado no exame de qualificação, este trabalho tinha como proposta inicial o estudo de esquemas numéricos para resolver as formulações hiperbólicas de valor de fronteira e de valor inicial para uma frente em movimento. Diante do que foi proposto e apresentado nessa dissertação, estamos convencidos de que os objetivos foram cumpridos.

Estudados neste trabalho, os esquemas numéricos para resolver as formulações hiperbólicas de valor de fronteira e de valor inicial que regem a propagação de uma frente, são baseados nas relações entre as equações de Hamilton-Jacobi e leis de conservação hiperbólicas. A correspondência direta entre essas duas classes de equações diferenciais acontece para o caso unidimensional onde uma equação unidimensional de Hamilton-Jacobi é transformada numa lei de conservação unidimensional, "integrando uma vez". Similaridades também existem para o caso multidimensional, e isto foi de ajuda na construção de esquemas numéricos para o caso onde o Hamiltoniano depende de mais de uma variável.

Assim sendo, estudamos duas classes de esquemas numéricos usados para integrar leis de conservação hiperbólicas com o intuito de aplicá-los em ambas formulaçães. As equações hiperbólicas estão relacionadas com a presença de soluções descontínuas e choques, e por isso foi necessário a introdução de noções de soluções fracas e condições de entropia. Para isso, 
estudamos esquemas com propriedades especiais, tais como, esquemas Upwind, Monótonos, Entropia e esquemas de Alta Resolução.

Esquemas de discretização utilizando diferenças centrais não produziram resultados satisfatórios, enquanto que, esquemas upwind que consistem na utilização de diferenças assimétricas de acordo com a direção de propagação da solução, possuem propriedades desejáveis para o tratamento de choques na solução. Resultados numéricos têm mostrado que esses esquemas são particularmente eficientes quando um choque ocorre em uma localizaçāo onde o fluxo muda de direção ou quando um fluxo supersônico torna-se subsônico.

Uma outra importante classe de esquemas para resolver as leis de conservação e consequentemente as equações de Hamilton-Jacobi é a classe dos esquemas monótonos discutidos por Crandall e Lions [8]. Infelizmente, esquemas monótonos são de primeira ordem de precisão, mas sendo conservativos, consistentes com a lei de conservação produzem a solução entrópica.

Estudamos esquemas clássicos de alta ordem, os quais mostraram-se inviáveis devido ao fato de produzirem oscilações espúrias na presença de descontinuidades. Para eliminarmos esse problema construímos esquemas de diferenças finitas com propriedades devidamente projetadas para evitar as oscilações e que não envolvem qualquer tratamento especial da descontinuidade, cuja propriedade essencial é deter o aparecimento das oscilaçōes no entorno das descontinuidades, os chamados TVD. Similarmente aos esquemas monótonos, esquemas TVD são de primeira ordem de precisão. Com a finalidade de utilizar esquemas com a precisão dos esquemas tradicionais e com propriedades TVD, foram projetados esquemas híbridos com tais propriedades, os quais são de segunda ordem em quase toda parte e não apresentam oscilações no entorno das descontinuidades. Embora possuindo boas propriedades, tais esquemas podem não produzir a solução entrópica.

Embora tenhamos estudado vários esquemas de discretização e algumas de suas principais propriedades conforme resumimos acima, na discretização das formulações utilizamos apenas as propriedades ou noções de esquemas upwind monótonos. Quando desejamos aumentar a precisão, utilizamos uma interpolaçāo essencialmente não-oscilatória ou ENO, os quais são esquemas TVD ligeiramente relaxados.

Das implementações que fizemos nas seções apropriadas utilizando esquemas da teoria de Conjuntos de Níveis para a formulação de valor inicial, podemos dizer que os resultados foram satisfatórios, pois forneceram as melhores soluções em comparação com as soluções 
fornecidas por esquemas clássicos. Também pudemos examinar a precisão de esquemas de primeira de ordem e de segunda ordem através da seção 5.3 .

\subsubsection{Perspectivas Futuras}

Como já foi mencionado no Resumo desta dissertação, a parte da teoria de Conjuntos de Níveis e Métodos de Marcha Rápida que estudamos poderá ser empregada para o rastreamento da interface de escoamentos multifásicos, e uma aplicação futura que poderá ser também feita é a determinação da fronteira de um domínio a partir de seus pontos interiores para aplicação no simulador de escoamentos multifásicos na área de mecânica de fluidos.

Pode-se ainda dar início aos estudos de algoritmos computacionais mais eficientes e mais rápidos que envolvem a resolução de ambas as formulações. O Método de Conjuntos de Niveis apresentado anteriormente é uma versão relativamente simples que pode ser facilmente programado. Entretanto não é particularmente rápido, nem faz uso de eficientes estruturas de dados e recursos computacionais existentes que o tornariam mais eficiente. Os Métodos de Marcha Rápida são algoritmos ou técnicas computacionais que permitem a resolução da formulação de valor de fronteira sem iteração com maior precisão e rapidez.

É importante dizer que algumas figuras encontradas nessa dissertação foram obtidas da referência [38], outras, foram construídas com PICTEX. Muitas figuras foram produzidas por programas feitos em $M A T L A B$ através da implementação de equações diferenciais parciais hiperbólicas utilizando os comandos plot, mesh (surf) e contour.

Essa dissertação foi escrita utilizando o editor de texto PCTeX32. 


\section{Referências Bibliográficas}

[1] Adalsteinsson, D. and Sethian, J.A., A Fast Level Set Method for Propagating Interfaces, J. Comput. Phys., 118, 2, pp.269-277, 1995.

[2] Adalsteinsson, D. and Sethian, J.A., A Level Set Approach to a Unified Model for Etching, Deposition, and Lithography I: Algorithms and Two-Dimensional Simulations, J. Comput. Phys., 120, pp.128-144, 1995.

[3] Adalsteinsson, D. and Sethian, J.A., A Lềel Set Approach to a Unified Model for Etching, Deposition, and Lithography II: Three-Dimensional Simulations, J. Comput. Phys., 122, pp.348-366, 1995.

[4] Barth, T.J. and Sethian J.A., Numerical Schems for the Hamilton-Jacobi and Level Set Equations on Triangualated Domains, J. Comput. Phys., 145, 1, pp.1-40, 1998.

[5] Castelo Filho, A.; Fortuna, A.O.; Cuminato, J.A.; Tomé, M.F.; Mangiavacchi, N.; Mckee, S., A Technique for Including Surface Tension Effects in Free Surface Flow Calculations, trabalho em produção pelo grupo de pesquisa do LCAD, USP-São Carlos.

[6] Crandall, M.G.; Evans L.C. and Lions P.L., Some Properties of Viscosity Solutions of Hamilton-Jacobi Equations, Trans. Amer. Math. Soc., 282, pp.487-502, 1984.

[7] Crandall, M.G.; Ishii H. and Lions P.L., User's Guide to Viscosity Solutions of HamiltonJacobi Equations, Trans. Amer. Math. Soc., 282, pp.487-502, 1984.

[8] Crandall, M.G. and Lions P.L., Two Approximations of Solutions of Second Order Partial Differential Equations, Bulletin of the American Mathematical Society, Vol. 27, No. 1, pp.1-67, 1992.

[9] Crandall, M.G. and Lions P.L., Viscosity Solutions of Hamilton-Jacobi Equations, Transactions of the American Mathematical Society, Vol. 227, No. 1, pp.1-42, 1983. 
[10] Crandall, M.G. and Majda A., Monotone Difference Approximations for Scalar Conservation Laws, Mathematics of Computation, Vol. 34, No. 149, pp.1-21, 1980.

[11] Cuminato, J.A.; Meneguette Junior, M., Discretização de Equações Diferenciais Parciais: Técnicas de Diferenças Finitas, ICMC-USP São Carlos SP.

[12] Engquist, B.; Osher, S., Stable and Entropy Sastisfying Aproximations for Transonic Flows Calculations, Math. Comp., 34, 45, 1980.

[13] Fortuna, A. O.; Técnicas Computacionais para Dinâmica dos Fluidos, Conceitos Básicos e Aplicações. Editora da Universidade de São Paulo. São Paulo 2000.

[14] Garabedian, P., Partial Differential Equations, Wiley, New York, 1964.

[15] Godlewsky, E; Raviart, P-A., Hyperbolic Systems of Conservation Laws, Mathematics \& Applications, SMAI, 1991.

[16] Godunov, S.K., Finite Difference Method for Numerical Computation of Discontinuous Solutions of the Equation of Fluid Dynamics, Mat. Shornick., 47, 271, 1959.

[17] Goodman, J.B. and LeVeque R.J.,A Geometric Approach to High Resolution TVD Schemes, SIAM J. Numer. Anal., Vol. 25, No. 2, 1988.

[18] Harten, A. and Lax, P.D., A Random Choice Finite Difference Scheme for Hyperbolyc Conservation Laws, SIAM J. Num. Anal., 18, 289, 1981.

[19] Harten, A. and Lax, P.D., Preliminary Results on the Extension of ENO Schemes to Two-Dimensional Problems, Lectures Notes Math., 1270, 23-40, 1987.

[20] Harten, A.; Osher, S.; Engquist, B.; Chakravarthy, S.R., Some esults on Uniformily Accurate Essentially Nonoscillatory Schemes, Appl. Num. Math., 2, pp. 347-377, 1986.

[21] Harten, A.; Osher, S.; Engquist, B.; Chakravarthy, S.R., Uniformily High Order Accurate Essentially Non-oscillatory Schemes, III, J. Comp. Phys., 71, pp. 231-303, 1987.

[22] Hopf, E., The equation $u_{t}+u u_{x}=\mu u_{x x}$, Commun. Pur. Appl. Math. 3: (3), 201-230, 1950.

[23] Iório, V., EDP Um Curso de Graduação, Coleção Matemática Universitária, IMPA, CNPq, 1991. 
[24] Lax, P.D., Nonlinear Hyperbolic Equations, Commun. Pur. Appl. Math. 6: (2), 231-258, 1953.

[25] Leer, B.V.. On The Relation Between the Upwind-Differencing Schemes of Godunov. Engquist-Osher and Roe, SIAM I. Sci. Stat. Comput., Vol, 5, No, 1. 1984.

[26] LeVeque, R.J., Numerical Methods for Conservation Laws. Birkhauser, Basel. 1992.

[27] Oleinik. O., Discontinuous Solutions of Nonlinear Differential Equations. Amer, Math. Soc. Transl. Ser, 2, 26:95, 1957.

[28] Osher. S., Riemann Solvers. The Entropy Condition, and Difference Aproximations. SIAM J. Anal.. Vol. 21, No, 2. 1984.

[29] Osher, S. and Sethian. J.A.. Fronts Propagating with Curvature-Dependent Speed: Algorithms Based on Hamilton-Jacobi Formulations. Jornal of Computational Physics. 79. pp, 12-49, 1988.

[30] Osher, S. and Shu, C.W.. High-Order Essentially Nonoscillatory Schemes for HamiltonJacobi Equations. SIAM J. Num. Anal., Vol, 28, No. 4, pp. 907-922, 1991.

[31] Osher. S. and Shu, C.W.. Efficient Implementation of Essencially Non-oscillatory Shock-Capturing Schemes. J. Comp. Phys., 77. pp. 439-471. 1988.

[32] Osher. S, and Shu. C.W.. Efficient Implementation of Essencially Non-oscillatory Shock-Capturing Schemes. II. J. Comp. Phys., 83, pp. 32-78. 1989.

[33] Roe. P.L., The Use of the Riemann Problem in Finite Difference Schemes. Proceedings of the Seventh International Conference on Numerical Methods in Fluid Dynamics, Lecture Note in Physics, 141. W.C. Reynolds and R.W. Mac Cormack eds.. Springer-Verlag. New York, 1981.

[34] Roe. P.L.. Aproximate Riemann Solvers. Parameter Vectors, and Difference Schemes. J. Comp. Phys., 43. 357. 1981.

[35] Sethian, J.A.. A Fast Marching Level Set Method for Monotonically Advancing-fronts. Proc. Nat. Acad. Sci.. 93. 4, pp.1591-1595. 1996. 
[36] Sethian, J.A., Curvature and the Evolution of Fronts, Comm. Math. Phys., 101, pp.487499, 1985.

[37] Sethian. J.A.. Fast Marching Methods, Siam Review. 41. 2. pp.199-235. 1999.

[38] Sethian. J.A.. Level Set Methods and Fast Marching Methods: Evolving Interfaces in Computational in Geometry. Fluid Mechanics, Computer Vision and Materials Sciences. Cambridge University Press. Cambridge, UK. 1998.

[39] Sethian, J.A.. Numerical Methods Propagating Fronts, in Variational Methods for Free Surface Interfaces, Eds. P. Concus and R. Finn, Springer-Verlag, NY, 1987.

[40] Sethian. J.A. and Strain. J.D.. Crystal Growth and Dendritic Solidification. J. Comp. Phys. 98. pp. 231-253. 1992.

(41) Sod, G.A.. Numerical Methods in Fluid Dynamics. Cambridge University

[42] Sweby, P.K.. High Resolution Schemes Using Flux Limiters for Hyperbolic Conservation Laws. SIAM J. Numer. Anal.. 21(5). pp. 995-1010. 1984.

[43] Thomas. J.W.. Numerical Partial Differential Equations: Finite Difference Methods. Texts in Applied Mathematics 22. Springer-Verlag, NY, 1995.

[44] Thomas, J.M.. Numerical Partial Differential Equations: Conservation Laws and Elliptic Equations. Texts in Applied Mathematics 33, Springer-Verlag. NY. 1999. 